\title{
Oscillatory flows in jet pumps
}

\section{Towards design guidelines for thermoacoustic applications}
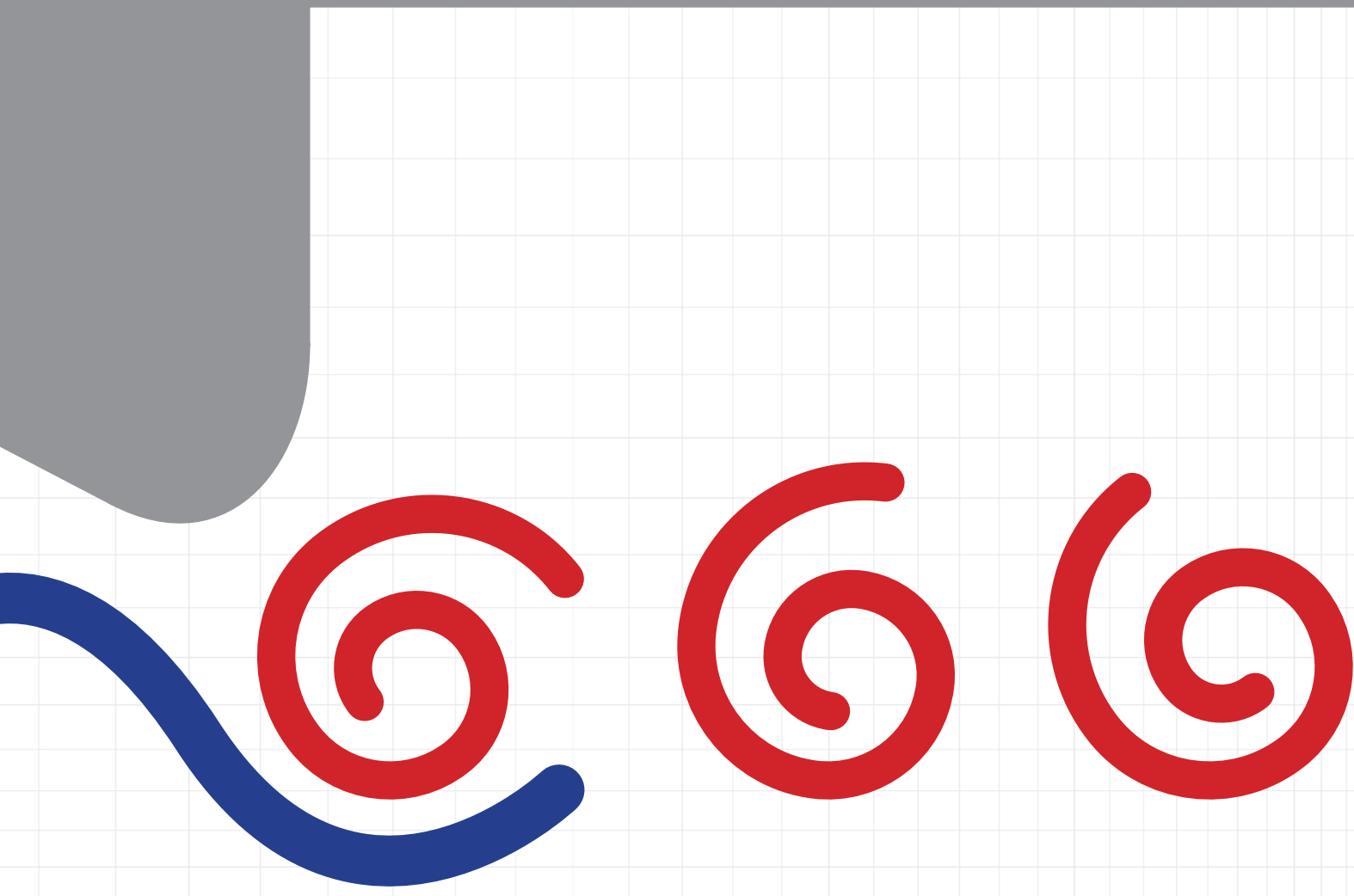

Joris Oosterhuis 



\section{OSCILLATORY FLOWS IN JET PUMPS}

Towards design guidelines for thermoacoustic applications

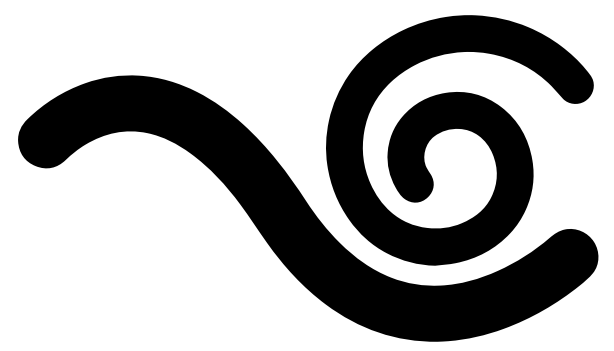


Samenstelling van de promotiecommissie:

Voorzitter

Prof. dr. G. P. M. R. Dewulf

Promotor

Prof. dr. ir. T. H. van der Meer

Leden

Prof. dr. ir. A. Hirschberg

Prof. dr. ir. H. W. M. Hoeijmakers

Prof. dr. ir. B. J. Boersma

Dr. G. Penelet

Dr. ir. J. C. H. Zeegers

D. A. Wilcox
Universiteit Twente

Universiteit Twente

Universiteit Twente

Universiteit Twente

Technische Universiteit Delft

Université du Maine, France

Technische Universiteit Eindhoven

Chart Industries, Inc.

\section{UNIVERSITY OF TWENTE.}

Faculty of Engineering Technology

Laboratory of Thermal Engineering

The work described in this thesis was financially supported by Bosch Thermotechnology and Rijksdienst voor Ondernemend Nederland as part of the EOS-KTO research program under project number KTOT03009.

Typeset with $\mathrm{AT}_{\mathrm{EX}}$.

Printed by Gildeprint Drukkerijen, Enschede.

Cover image: illustration of the vortex shedding from a jet pump due to the interaction with an acoustic wave.

Copyright (C) 2016 Joris Oosterhuis

ISBN: $\quad$ 978-90-365-4063-6

DOI: $\quad 10.3990 / 1.9789036540636$

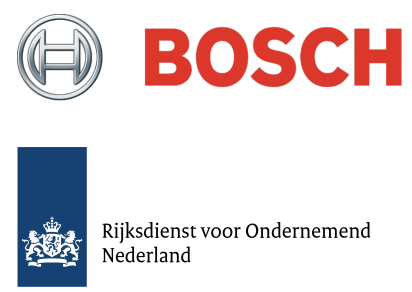




\title{
OSCILLATORY FLOWS IN JET PUMPS
}

TOWARDS DESIGN GUIDELINES FOR THERMOACOUSTIC APPLICATIONS

\section{PROEFSCHRIFT}

\author{
ter verkrijging van \\ de graad van doctor aan de Universiteit Twente, \\ op gezag van de rector magnificus, \\ prof. dr. H. Brinksma \\ volgens besluit van het College voor Promoties \\ in het openbaar te verdedigen \\ op vrijdag 29 april 2016 om 16:45 uur
}

door

Joris Pieter Oosterhuis

geboren op 15 november 1987

te Breda 
Dit proefschrift is goedgekeurd door:

Prof. dr. ir. T. H. van der Meer 


\section{Summary}

Thermoacoustic engines are an interesting alternative to conventional heat engines (such as Stirling engines) due to the absence of moving parts in the hot region and the small temperature difference required to operate. These engines can provide a durable solution in, for example, waste heat recovery applications. Using a traveling wave based configuration, consisting of a toroidal geometry, thermal-to-acoustic efficiencies of up to $30 \%$ have been obtained. However, the traveling wave configuration has a major disadvantage: due to the closed looped geometry a time-averaged mass flow, known as "Gedeon streaming", can occur. This type of acoustic streaming can lead to a drastic reduction in efficiency or even prevent the engine from running. Therefore, control of Gedeon streaming is essential in the development of traveling wave thermoacoustic engines.

A solution to avoid Gedeon streaming is the application of a jet pump, which is a component with one or more tapered holes. The oscillatory flow through such an asymmetric geometry results in a time-averaged pressure drop across the jet pump. By balancing this time-averaged pressure drop with the pressure drop that exists across the regenerator of the thermoacoustic device, Gedeon streaming can be suppressed. In this thesis, the oscillatory flow in jet pumps is analyzed. The jet pump performance in terms of the time-averaged pressure drop and acoustic power dissipation is studied as a function of the jet pump geometry, the operating frequency and the amplitude of the acoustic wave. Five different studies are performed and presented as separate chapters in this thesis.

Using a two-dimensional axisymmetric computational fluid dynamics (CFD) model, the laminar oscillatory flow through single-hole jet pump geometries is simulated. Four different flow regimes are distinguished based on the flow features identified, such as vortex ring formation and flow separation. The occurrence of the flow regimes is subsequently linked to the simulated jet pump performance. The jet pump diameter, taper angle, length and edge curvature are varied independently and scaling parameters are introduced to predict the performance and flow regime as a function of the jet pump geometry. Based on this, design guidelines for jet pumps in laminar oscillatory flows are formulated.

Flow separation from the inner jet pump wall is shown to have a large negative impact on the performance of a jet pump. In laminar oscillatory flows, the onset of flow separation is directly related to the acoustic displacement amplitude in combination with the jet pump diameter and taper angle. This onset value is confirmed experimentally using hot-wire anemometry in both laminar and turbulent oscillatory flows. 
The process of flow separation is characterized in a separate numerical study and a design adjustment is proposed that can significantly reduce the flow separation. By introducing a smooth curved transition from the jet pump small opening towards its tapered surface, the onset of flow separation is shifted to larger displacement amplitudes and the duration of the separated flow is reduced. This greatly enhances the effectiveness and robustness of jet pumps in laminar flows. To make the proposed jet pump designs more compact without affecting their performance, it is shown to what extent the big opening diameter can be reduced before local minor losses influence the jet pump performance.

The effect turbulence has on the performance of a jet pump is investigated in an experimental study. By measuring the time-averaged pressure drop and acoustic power dissipation, it is observed that the large performance decrease induced by flow separation is mitigated in turbulent flows. Instead of a rapid decrease in the dimensionless time-averaged pressure drop after the onset of flow separation, the dimensionless time-averaged pressure drop has the tendency to stabilize in the turbulent flow regime. Hot-wire anemometry is used to characterize the level of turbulence. The critical Reynolds number for oscillatory pipe flows is found to be a correct predictor for turbulence in jet pumps as well.

For compact thermoacoustic devices it is important to design a compact jet pump geometry with a minimal reduction in performance. Decreasing the length of a jet pump by increasing its taper angle is shown to directly facilitate the occurrence of flow separation and should be avoided. Alternatively, the size of a jet pump can be reduced by employing multiple smaller orifices instead of one single hole. Doing so largely reduces the jet pump length while both the total cross-sectional area and the taper angle remain unchanged. The effect of this design approach on the jet pump performance is investigated experimentally. Although a significant performance decrease is measured when increasing the number of holes from 1 up to 16 holes, the time-averaged pressure drop remains much higher compared to compact geometries with large taper angles. The decrease in time-averaged pressure drop for multiple hole jet pumps is shown to be caused by the smaller diameter of the individual orifices rather than to the interaction of flow between adjacent orifices.

The studies in this thesis show the relation between oscillatory flow features and the performance of jet pumps. Based on this, jet pump design guidelines have been formulated for laminar oscillatory flows. Flow separation is identified as a main source of performance loss in jet pumps and can be avoided by introducing a smooth transition to the tapered inner surface. Compact jet pump designs can be realized by using multiple smaller tapered holes, but this is accompanied by a slight reduction in performance due to the smaller diameter of the individual holes. Identifying and understanding the flow phenomena in jet pumps is shown to be the key to more reliable design calculations for jet pumps in thermoacoustic applications. 


\section{Samenvatting}

Thermoakoestische motoren zijn een interessant alternatief voor conventionele warmtemotoren (zoals Stirling motoren) door het ontbreken van bewegende delen in het warme gedeelte en het kleine temperatuurverschil dat nodig is om te functioneren. Deze motoren zijn een duurzame oplossing voor bijvoorbeeld toepassingen waar restwarmte beschikbaar is. Met een lopende-golfconfiguratie, bestaande uit een torusvormige geometrie, zijn rendementen tot $30 \%$ behaald in de omzetting van thermische naar akoestische energie. Deze lopende-golfconfiguratie heeft echter een belangrijk nadeel: in de kringvormige geometrie kan een tijdgemiddelde stroming (zgn. "Gedeon streaming") voorkomen. Dit type akoestische streaming kan leiden tot een drastische rendementsverlaging of er zelfs voor zorgen dat de motor niet meer werkt. Het voorkomen van Gedeon streaming is daarom een belangrijk aandachtspunt bij de ontwikkeling van lopende golf thermoakoestische motoren.

Een oplossing om Gedeon streaming tegen te gaan, is de toepassing van een jet pomp, een component met één of meerdere tapstoelopende gaten. De oscillerende stroming door zo'n asymmetrische component leidt tot een tijdgemiddelde drukval over de jet pomp. Wanneer deze tijdgemiddelde drukval even groot is als de drukval over de regenerator van de thermoakoestische motor kan de Gedeon streaming tegengegaan worden. In dit proefschrift is de oscillerende stroming door jet pompen geanalyseerd. Door naar de tijdgemiddelde drukval en de dissipatie van akoestische energie te kijken, zijn de prestaties van jet pompen bestudeerd als functie van de geometrie, de werkfrequentie en de amplitude van de akoestische golf. Vijf verschillende studies zijn uitgevoerd en zijn te vinden als aparte hoofdstukken in dit proefschrift.

De laminaire oscillerende stroming door een jet pomp geometrie met één gat is gesimuleerd met een tweedimensionaal axisymmetrisch numeriek stromingsmodel (CFDmodel). Op basis van de stromingskenmerken worden vier verschillende stromingsregimes onderscheiden, zoals de vorming van ringvortices en stromingsloslating. Het optreden van deze stromingsregimes is vervolgens gerelateerd aan de jet pomp prestaties. De diameter, de conushoek, de lengte en de afronding van de jet pomp zijn onafhankelijk van elkaar gevarieerd. Verschillende schalingsparameters zijn geïntroduceerd om de prestaties en het stromingsregime te voorspellen als functie van de jet pomp geometrie. Op basis hiervan zijn ontwerprichtlijnen voor jet pompen in laminaire oscillerende stromingen geformuleerd.

Stromingsloslating vanaf de binnenwand van de jet pomp blijkt een grote negatieve invloed op de prestaties van de jet pomp te hebben. In laminaire oscillerende stromingen is het optreden van loslating direct gerelateerd aan de akoestische verplaatsingsam- 
plitude in combinatie met de diameter en conushoek. Het optreden van loslating is experimenteel gevalideerd met hot-wire anemometrie in zowel laminaire als turbulente oscillerende stromingen. Het proces van loslating is gekarakteriseerd in een numerieke studie en een aanpassing in het ontwerp is voorgesteld die de loslating significant kan beperken. Door het introduceren van een vloeiende kromming tussen de kleine opening van de jet pomp en het tapstoelopende oppervlak, is het optreden van loslating uitgesteld tot grotere verplaatsingsamplitudes en is de tijdsduur van de loslating verkort. Dit leidt tot een sterke verbetering van de effectiviteit en robuustheid van jet pompen in laminaire stromingen. Om een jet pomp compacter te ontwerpen zonder de prestaties te beïnvloeden, is geanalyseerd in welke mate de diameter van de grote opening gereduceerd kan worden voordat lokale drukverliezen de prestaties van de jet pomp aantasten.

Het effect van turbulentie op de prestaties van een jet pomp is onderzocht in een experimentele studie. De grote afname in de prestaties van de jet pomp vanaf het optreden van stromingsloslating is minder sterk in turbulente stromingen. In plaats van een grote afname van de dimensieloze tijdgemiddelde drukval vanaf het optreden van loslating, neigt de dimensieloze tijdgemiddelde drukval te stabiliseren in het turbulente regime. Hot-wire anemometrie is gebruikt om de mate van turbulentie te karakteriseren. Het kritieke Reynoldsgetal voor oscillerende pijpstromingen blijkt ook een correcte voorspeller te zijn voor turbulentie in jet pompen.

Voor compacte thermoakoestische motoren is het belangrijk om een compacte jet pomp geometrie te ontwerpen en tegelijkertijd de prestaties zo min mogelijk te beinvloeden. Een jet pomp korter maken door de conushoek te vergroten heeft een direct nadelig effect op het optreden van stromingsloslating en moet daarom vermeden worden. In plaats daarvan kan de lengte van een jet pomp verkleind worden door meerdere kleinere parallelle gaten te gebruiken in plaats van één gat. Hierdoor wordt de lengte sterk gereduceerd terwijl zowel het totale dwarsdoorsnedeoppervlak als de conushoek gelijk blijven. Het effect van deze ontwerpaanpak op de jet pomp prestaties is experimenteel onderzocht. Er is een significante reductie in de prestaties van de jet pomp gemeten als het aantal gaten vergroot wordt van één tot zestien gaten. Echter, de prestaties blijven aanzienlijk beter dan wanneer de conushoek vergroot zou worden. De afname in de tijdgemiddelde drukval voor jet pompen met meerdere gaten wordt veroorzaakt door de kleinere diameter van de individuele openingen in plaats van interactie van stroming tussen aangrenzende openingen.

De studies in dit proefschrift laten de relatie zien tussen oscillerende stromingsverschijnselen en de prestaties van jet pompen. Op basis hiervan zijn jet pomp ontwerprichtlijnen geformuleerd voor laminaire oscillerende stromingen. Stromingsloslating is de belangrijkste bron van prestatievermindering in jet pompen en kan vermeden worden door een vloeiende overgang te gebruiken naar het tapstoelopende oppervlak van de jet pomp. Compacte jet pomp geometriën kunnen gerealiseerd worden door meerdere conische gaten te gebruiken maar dit leidt tot een geringe reductie in de jet pomp prestaties door de kleinere diameter van de individuele gaten. Het identificeren en begrijpen van stromingsverschijnselen in jet pompen is essentieel om betrouwbare ontwerpberekeningen te kunnen maken voor thermoakoestische toepassingen. 


\section{Contents}

1 Introduction 1

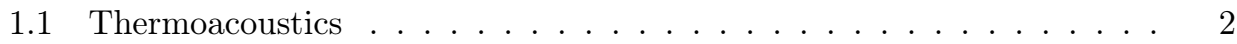

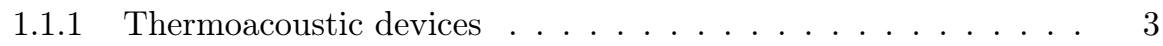

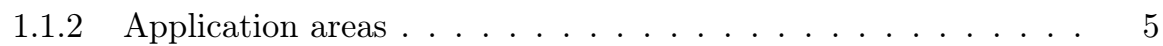

1.2 Gedeon streaming . . . . . . . . . . . . . . . . . 5

1.3 Jet pumps . . . . . . . . . . . . . . . . . . 6

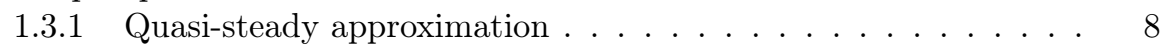

1.3.2 Literature review . . . . . . . . . . . . . . . . . . 9 9

1.4 Thesis outline . . . . . . . . . . . . . . . . . . 10

2 Jet pump flow regimes 13

2.1 Introduction . . . . . . . . . . . . . . . . . . . 13

2.2 Modeling . . . . . . . . . . . . . . . . . . . . 14

2.2.1 Geometry ............................ 14

2.2 .2 Numerical setup . . . . . . . . . . . . . . . . . 15

2.2.3 Time-domain impedance boundary condition . . . . . . . . . 16

2.2.4 Data analysis . . . . . . . . . . . . . . . . 17

2.2.5 Computational mesh . . . . . . . . . . . . . . . 17

2.3 Results and discussion . . . . . . . . . . . . . . . . . . . . . . . 18

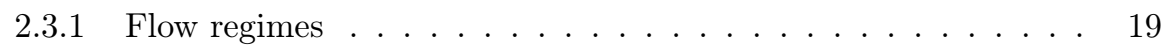

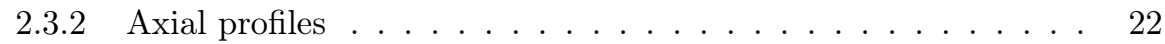

2.3.3 Jet pump performance . . . . . . . . . . . . . . . . . . 25

2.4 Conclusions . . . . . . . . . . . . . . . . . . . . . 28

3 Geometric parameter study $\quad 31$

3.1 Introduction . . . . . . . . . . . . . . . . . . . . . 31

3.2 Modeling . . . . . . . . . . . . . . . . . . . . . . . . . . . . . . . . . . .

3.2.1 Simulation of flow separation . . . . . . . . . . . . . . . . . 32

3.2 .2 Jet pump geometries . . . . . . . . . . . . . . . . . . . . . . . . . . . . . . . . 33

3.3 Results and discussion . . . . . . . . . . . . . . . . . . 35

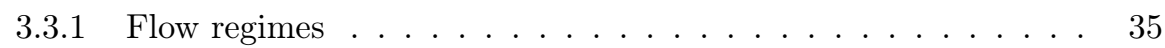

3.3.2 Jet pump performance . . . . . . . . . . . . . . . 37

3.3.3 Influence of waist curvature . . . . . . . . . . . . . . . . . . . . . . . . . . 41

3.4 Conclusions . . . . . . . . . . . . . . . . . . . . . . 46

4 Reducing flow separation in jet pumps 49

4.1 Introduction . . . . . . . . . . . . . . . . . 49 


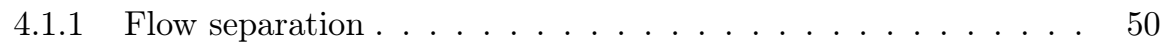

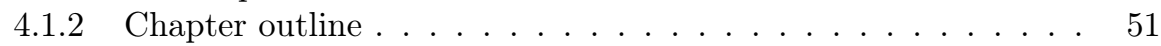

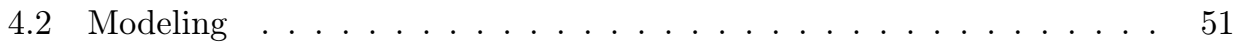

4.2.1 Computational mesh . . . . . . . . . . . . . . . . 52

4.2 Laminar flow . . . . . . . . . . . . . . . . . . . . . . . 52

4.3 Characterization of flow separation . . . . . . . . . . . 53

4.3.1 Boundary layer breakaway . . . . . . . . . . . . . 53

4.3.2 First flow reversal . . . . . . . . . . . . . . . . . 55

4.3.3 Effect of flow separation on performance . . . . . . . . . 56

4.4 Reduction of flow separation . . . . . . . . . . . . . . . 58

4.4.1 Increased transition length . . . . . . . . . . . . . . 58

4.4 .2 Compact designs . . . . . . . . . . . . . . . . 63

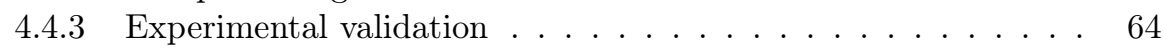

4.5 Conclusions . . . . . . . . . . . . . . . . . . . 65

5 Turbulence and flow separation $\quad 67$

5.1 Introduction . . . . . . . . . . . . . . . . . 67

5.2 Experimental setup . . . . . . . . . . . . . . . 68

5.2.1 Pressure measurement system . . . . . . . . . . . . . 69

5.2 Data analysis . . . . . . . . . . . . . . . . . 69

5.3 Jet pump performance . . . . . . . . . . . . . . . . . 70

5.4 Flow separation and vortex propagation . . . . . . . . . . . 73

5.4 Velocity time traces . . . . . . . . . . . . . . 75

5.4.2 Identification of flow separation and vortex propagation . . . . 76

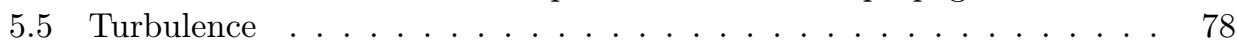

5.6 Conclusions . . . . . . . . . . . . . . . . . . . 81

6 Jet pumps with multiple orifices $\quad 83$

6.1 Introduction . . . . . . . . . . . . . . . . . . . 83

6.2 Experimental setup . . . . . . . . . . . . . . . . . . 84

6.3 Jet pump performance . . . . . . . . . . . . . . . . 85

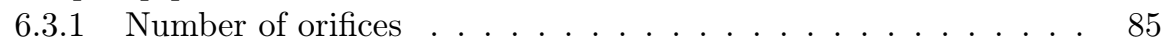

6.3 .2 Orifice spacing . . . . . . . . . . . . . . . . 90

6.4 Flow visualization . . . . . . . . . . . . . . . . . . . . . 91

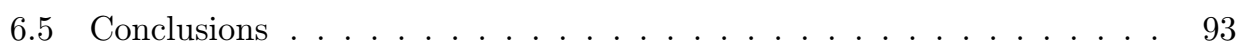

7 Conclusions $\quad 95$

7.1 Jet pump performance in laminar oscillatory flows . . . . . . . . . . . 95

7.2 Characterization and reduction of flow separation . . . . . . . . . . . 96

7.3 Influence of turbulence on the jet pump performance . . . . . . . . . . 97

7.4 Jet pumps with multiple orifices . . . . . . . . . . . . . . . . . . . 97

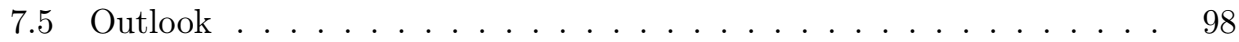

\section{Appendices}

A Time-domain impedance boundary condition 101

A.1 Introduction . . . . . . . . . . . . . . . . . . . . 101 
A.2 Theory and implementation . . . . . . . . . . . . . . . 102

A.2.1 Non-reflective boundary condition . . . . . . . . . . . . . . . 102

A.2.2 Time-domain impedance boundary condition . . . . . . . . . . 104

A.2.3 Implementation . . . . . . . . . . . . . . . . . . . . . . . . . . 104

A.3 One-dimensional validation . . . . . . . . . . . . . . . . . . . 104

A.4 Two-dimensional validation . . . . . . . . . . . . . . . . . 107

A.4.1 Low reduced frequency model . . . . . . . . . . . . . . . . . 107

A.4.2 Numerical setup . . . . . . . . . . . . . . . . . . . 108

A.4.3 Results ....................... 109

B Pressure measurement and calibration 111

B.1 Signal sampling . . . . . . . . . . . . . . . . . . . . 112

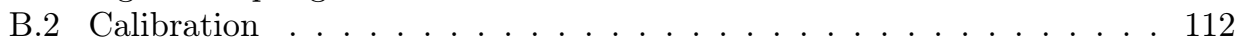

B.2.1 Sensor sensitivity . . . . . . . . . . . . . . . . . . 113

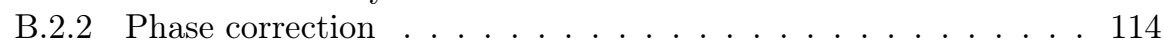

B.2.3 Linearity . . . . . . . . . . . . . . . . . . . . . 115

B.2.4 Stability and repeatability . . . . . . . . . 116

C Acoustic modeling of experimental setup 117

C.1 Introduction . . . . . . . . . . . . . . . . . . 117

C.2 Model ................................ 117

C.2.1 Low reduced frequency model . . . . . . . . . . . . . . . . . 118

C.2.2 Coupled COMSOL model . . . . . . . . . . . . . . . . . . . 119

C.2.3 From measured pressure to velocity amplitude . . . . . . . . . 119

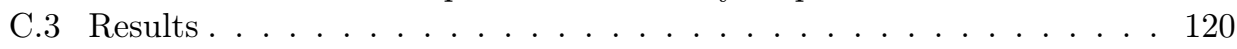

C.3.1 Effect of jet pump geometry . . . . . . . . . . . . . . . . 122

C.4 Discussion . . . . . . . . . . . . . . . . . . . . 123

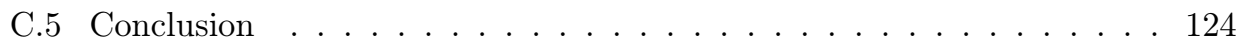

$\begin{array}{ll}\text { D Surface roughness } & \mathbf{1 2 5}\end{array}$

D.1 Introduction . . . . . . . . . . . . . . . . . . 125

D.2 Results. . . . . . . . . . . . . . . . . . 126

D.3 Conclusion . . . . . . . . . . . . . . . . . . 127

$\begin{array}{lr}\text { List of symbols } & 129\end{array}$

$\begin{array}{ll}\text { Bibliography } & 133\end{array}$

$\begin{array}{ll}\text { Curriculum vitae } & 141\end{array}$

$\begin{array}{ll}\text { Dankwoord } & 145\end{array}$ 


\section{Introduction}

Oscillatory fluid flows exhibit many features that can be very fascinating and are not found in steady flows. Probably the most well-known example is that of a smoke ring shown in Fig. 1.1a. A smoke ring is actually a rotating ring-shaped piece of smoke, also called a vortex ring. Vortex rings are formed when an oscillating or pulsating flow interacts with a ring-shaped structure, such as an orifice. A schematic representation of this process is shown in Fig. 1.1b. At the edge of the orifice, the flow separates (1) and rolls up into a vortex ring (2), which subsequently propagates away from the orifice (3). ${ }^{1}$ Similar vortical ring structures can be observed in nature from, for instance, dolphins blowing underwater bubble rings or during volcano eruptions. ${ }^{2-4}$ In all these situations, an oscillating or pulsating flow interacts with an orifice-like geometry.
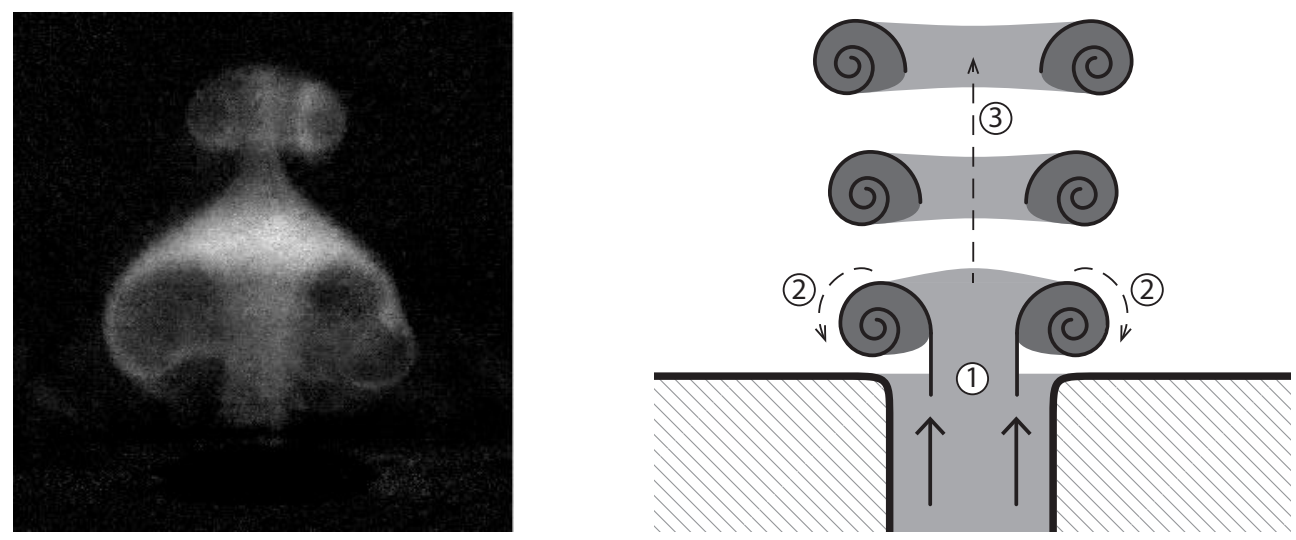

(a)

(b)

Figure 1.1: (a) Side-view of vortex rings emitted from a jet pump sample. (b) Schematic illustration of the vortex ring formation process from the exit of a smoke filled cavity. 
Vortex rings are not only suitable to astonish people, they also have useful applications in flow control. ${ }^{1}$ So-called synthetic jet actuators, driven by an oscillating membrane, generate trains of vortices. The expulsion of vortex rings results in a jet flow that can be used to control flow separation and turbulence, which has drawn attention from the aircraft industry. ${ }^{1,5}$ The generation of vortex rings is also associated with flow losses (i.e., pressure drop and energy dissipation), which are generally undesired. However, an application which actually makes use of these flow losses and where vortex rings play an important role, is a jet pump. This passive device can be used to generate a pressure difference in oscillatory flows and finds its application as a "pump" in thermoacoustic devices. ${ }^{6-8}$ Although it might sound odd at first, introducing flow losses in the right way is essential for certain thermoacoustic applications to operate and highly increases the efficiency of these devices. By unraveling the exact working of jet pumps, design guidelines for jet pumps in thermoacoustic devices can be developed, which is the aim of this study. Before explaining the motivation for using a jet pump in Section 1.2 and its working principle in Section 1.3, the application of thermoacoustic devices and the underlying thermoacoustic effect require some introduction.

\subsection{Thermoacoustics}

While acoustic waves are commonly considered to be only pressure and velocity oscillations, the density and temperature also vary proportional to the pressure perturbation. The reverse is also true: by imposing a temperature variation to a volume of gas, an acoustic wave can be amplified. This thermoacoustic effect was first described qualitatively in 1887 by what is now known as the Rayleigh criterion:

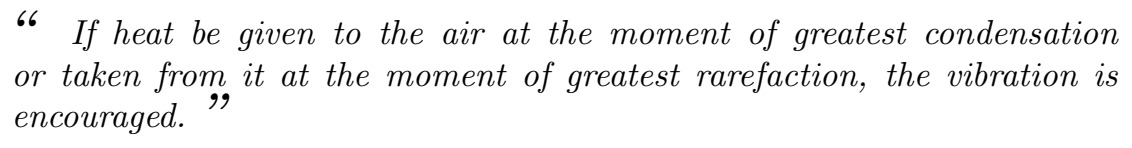
or taken from it at the moment of greatest rarefaction, the vibration is encouraged.

\section{- Lord Rayleigh ${ }^{9}$}

Temperature variations associated with acoustic waves are typically very small and often neglected. For example in human speech ( $40 \mathrm{~dB}$ to $60 \mathrm{~dB})$, the temperature oscillation is in the order of $10 \mu \mathrm{K}$. However, in applications where gas undergoes large temperature or pressure variations, the thermoacoustic effect can have both desired and undesired consequences. Some of these cases will be outlined in the following brief historical perspective.

Carl Sondhauss was among the first to describe the thermoacoustic effect, which occurred when glass blowers would blow a bulb at the end of a narrow tube. ${ }^{10}$ The gas inside the tube undergoes a temperature variation and a spontaneous acoustic oscillation can occur emitting sound. A similar system is the "Rijke tube", which is nowadays a common lecture demonstration of the thermoacoustic effect and was first developed by Pieter Rijke in 1859. ${ }^{11}$ In an open tube with a hot wire gauze a standing acoustic wave is enforced using natural convection. In gas turbines, the same thermoacoustic effect can have a devastating effect on the system. ${ }^{12}$ Thermoacoustic oscillations can also be initiated by combustion instabilities. When these oscillations 


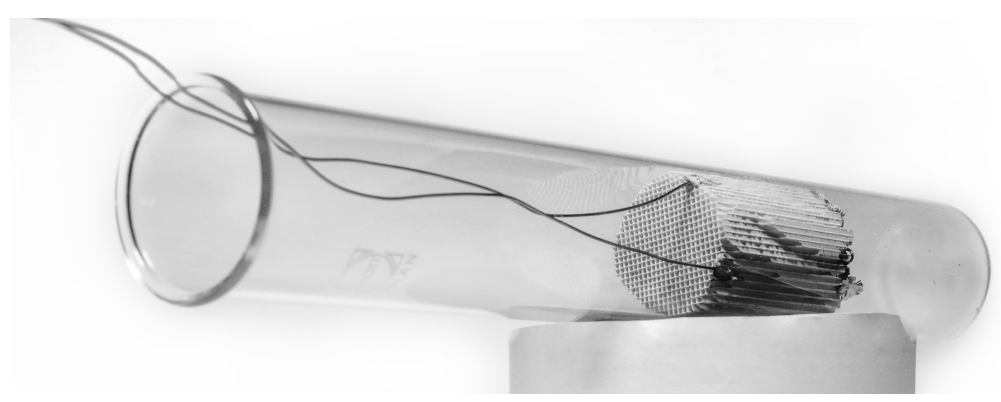

Figure 1.2: Assembled acoustic laser demo device consisting of a glass tube, a ceramic stack, and a heater wire located at the backside of the stack.

couple with structural modes, high amplitude oscillations that can damage or destroy systems can be reached. However, when properly designed, the thermoacoustic effect can also be used for energy conversion purposes. The acoustic power generated is a form of mechanical power and can be converted into electricity. ${ }^{13,14}$

\subsubsection{Thermoacoustic devices}

The conversion of heat into acoustic power and vice versa designates thermoacoustics as a potential working principle for heat engines (converting heat into acoustic power), or heat pumps and refrigerators (converting acoustic power into heating or refrigeration). These thermoacoustic devices are an interesting alternative to conventional heat engines or refrigerators due to the lack of moving parts in the hot region and the relatively low temperature difference required to operate. Furthermore, in contrast to conventional cooling cycles no harmful greenhouse gases are involved. Mostly helium, argon or air are used as a working fluid. ${ }^{15}$

A major step towards the design of thermoacoustic devices was the development of a linear thermoacoustic theory by Nikolaus Rott. ${ }^{16,17}$ However, it was not until the 1980's that the thermoacoustic effect was employed for developing practical applications, when advances were made at the Los Alamos National Laboratory. ${ }^{18}$ Initially, most work focused on the development of devices based on a standing wave phasing. The main difference with the early systems of Sondhauss and Rijke, ${ }^{10,11}$ is the application of a porous material (a "stack") to enhance the heat transfer to the working fluid. Probably the most simple standing wave thermoacoustic engine is the "acoustic laser" demo kit, which is shown in Fig. 1.2 and was developed in the Thermoacoustic Refrigeration group at Penn State University. ${ }^{19}$ A glass tube, a piece of porous ceramic material, and an electric heater wire is all that is needed to generate monotone sound emitted at about $130 \mathrm{~dB}$.

Despite its simplicity, standing wave thermoacoustic devices have one disadvantage. In a standing wave an imperfect thermal contact in the stack is required to obtain the correct phasing between the acoustic wave and heat transfer for the Rayleigh criterion to be satisfied. The imperfect thermal contact intrinsically leads to low theoretical 


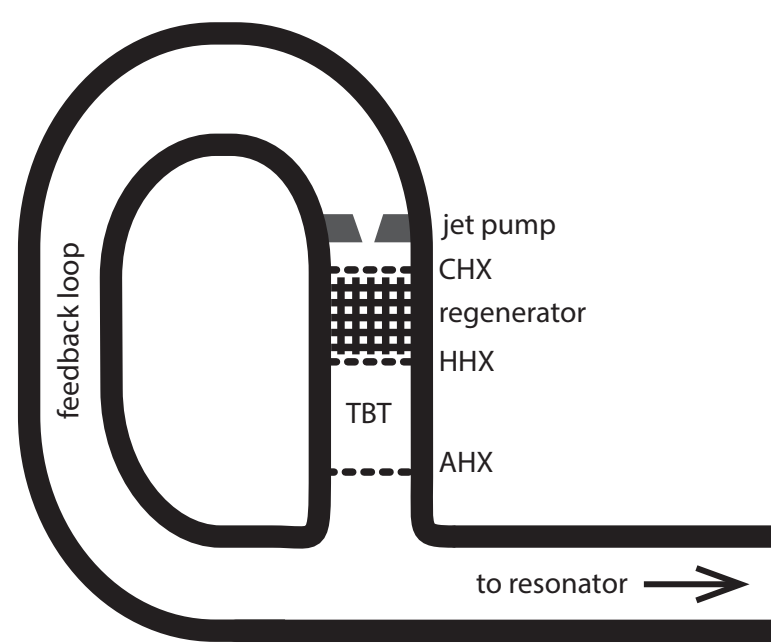

(a)

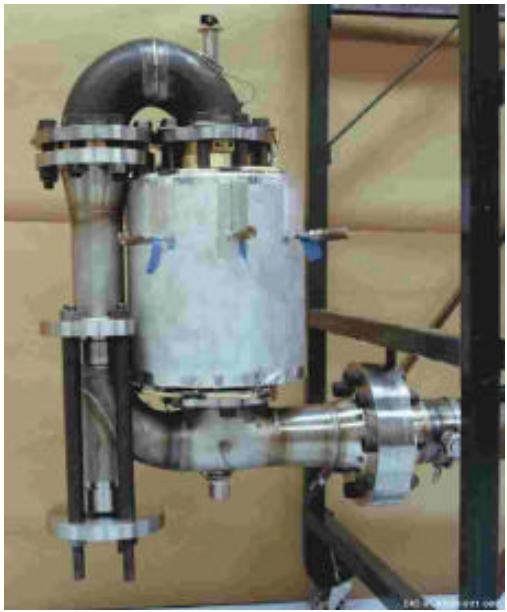

(b)

Figure 1.3: (a) Illustration of a traveling wave thermoacoustic engine topology designed by Backhaus and Swift. ${ }^{7}$ An acoustic wave is amplified due to the temperature difference across the regenerator. The temperature difference is maintained by the cold heat exchanger (CHX) and the hot heat exchanger (HHX). The secondary ambient heat exchanger (AHX) ensures a constant mean temperature in the rest of the engine and is thermally insulated from the hot heat exchanger by the thermal buffer tube (TBT). (b) Photograph of the same engine from Wollan et al. ${ }^{24}$

efficiencies. Ceperley realized that by using traveling waves (i.e., pressure and velocity oscillating in phase) this deficiency can be overcome. ${ }^{20} \mathrm{~A}$ reversible Stirling cycle is the underlying thermodynamic cycle and imperfect thermal contact is no longer a requirement in the traveling wave thermoacoustic devices. The stack can now be replaced by a porous structure with much smaller pores and enhanced heat transfer, which is called the "regenerator". Hence, much higher theoretical efficiencies can be obtained compared to standing wave thermoacoustic devices.

A major breakthrough in thermoacoustic technology was made when Backhaus and Swift, ${ }^{7}$ and at about the same time De Blok, ${ }^{21}$ applied the traveling wave concept in the design of their engines. The engine of Backhaus and Swift reached a thermal-toacoustic efficiency of $30 \% .{ }^{22}$ Their system is shown schematically in Fig. 1.3a and has been exemplary for many traveling wave thermoacoustic systems developed to date. The core of the engine is formed by the regenerator, enclosed by a hot- and cold- heat exchanger to create the temperature difference required to amplify the acoustic wave. A feedback loop is used to realize the traveling wave phasing and along the resonator an acoustic load can be installed to extract acoustic power from the system. This acoustic power can subsequently be converted to electricity, ${ }^{13,14}$ or used to drive a coupled thermoacoustic system for heat pumping or refrigeration purposes. ${ }^{23}$ 


\subsubsection{Application areas}

Thermoacoustic devices have been constructed for various applications and different heat sources. Several solar powered thermoacoustic engines or refrigerators have been developed. ${ }^{23,25-27}$ Due to the low temperature difference required for operation, waste heat recovery is an interesting field of application for thermoacoustics. ${ }^{25,28-31}$ Motivated by the absence of moving parts and relatively loose tolerances compared to other heat engines, applications where either robustness or long-term reliability is important have been investigated. For example, outer space applications for cooling or electricity production. ${ }^{13,32-35}$ For electricity production in rural areas, robustness and cheap fabrication costs are essential. The potential of thermoacoustic technology for this goal has been shown in several projects. ${ }^{36-39}$ A field where thermoacoustics finds numerous applications is refrigeration. Both domestic systems ${ }^{40,41}$ as well as cryogenic devices are developed, such as for gas liquefaction. ${ }^{24,42}$ Other applications include gas separation and remote sensing technologies. ${ }^{43-47}$ More examples of thermoacoustic systems can be found in the review articles of Swift, Garrett and Jin. ${ }^{48-50}$

\subsection{Gedeon streaming}

Despite the high theoretical efficiencies, the traveling wave thermoacoustic configuration has one major disadvantage: due to the closed-loop geometry (see Fig. 1.3a) a time-averaged mass flow, known as "Gedeon streaming", can occur. ${ }^{51}$ Using a perturbation expansion of density $\rho$ and volume flow rate $U$, the time-averaged mass flow $\dot{m}_{2}$ can be written as ${ }^{15,51}$

$$
\dot{m}_{2}=\frac{1}{2} \Re\left(\widetilde{\rho}_{1} U_{1}\right)+\rho_{0} U_{2}
$$

This type of acoustic streaming leads to undesired convective heat transport that has a detrimental effect on the efficiency of closed-loop thermoacoustic devices. The associated heat loss is given $b^{7}$

$$
\dot{Q}=\dot{m}_{2} C_{p}\left(T_{h}-T_{c}\right)
$$

with $T_{h}$ and $T_{c}$ the temperatures at the hot and cold heat exchanger in Fig. 1.3a, respectively. As an indication, when no measures are taken to ensure $\dot{m}_{2}=0$, the heat loss due to Gedeon streaming can reach up to 2.5 times the total delivered acoustic power. ${ }^{7}$ Control of Gedeon streaming is therefore crucial to achieve efficient operation in closed-loop thermoacoustic devices.

The first term on the right hand side of Eq. 1.1 is proportional to the transported acoustic power and must be nonzero in traveling wave thermoacoustic devices. To cancel the Gedeon streaming, two different strategies can be applied: physically blocking the flow path for the Gedeon streaming, or canceling the time-averaged mass flow $\dot{m}_{2}$ by imposing a counteracting flow $U_{2}$ such that Eq. 1.1 equals zero. ${ }^{6}$ 

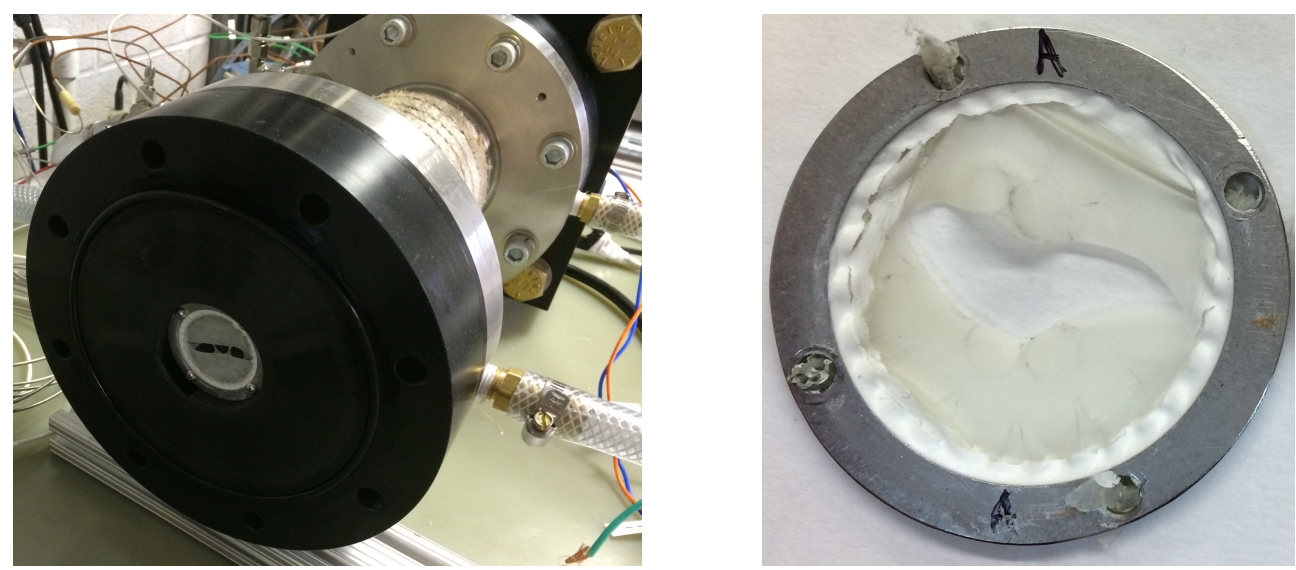

(a)

(b)

Figure 1.4: Membrane in a compact traveling wave thermoacoustic engine (a) torn after operation at $D r \approx 0.05$, (b) shows a close-up of the membrane after use. ${ }^{52}$

Blocking the flow path for the Gedeon streaming can be achieved by installing a membrane. Applied correctly, the membrane is a very simple solution as it provides a physical barrier that makes it impossible for there to exist a nonzero mass flux within the closed-loop. Because the membrane is acoustically transparent, it dissipates negligible acoustic power. Although successful operation has been reported using a membrane, ${ }^{28}$ this method is a potential threat to the long-term reliability of thermoacoustic engines. ${ }^{6}$ Recent experiments have confirmed that these membranes are susceptible to tear when operating at large acoustic amplitudes. ${ }^{52}$ Fig. 1.4 shows the result after operating at a pressure amplitude of approximately $5 \%$ of the mean pressure.

\subsection{Jet pumps}

As an alternative to using membranes, Gedeon streaming can be suppressed by imposing a counteracting mean flow. Swift et al. proposed making use of an asymmetry in hydrodynamic end effects to generate a time-averaged pressure drop. ${ }^{6}$ A geometry that will create such a time-averaged pressure drop in an oscillating flow is called a jet pump. The time-averaged pressure drop that the jet pump has to fulfill for $\dot{m}_{2}=0$, can be estimated by calculating the pressure difference across the regenerator of a traveling wave thermoacoustic device. Similarly following Eq. 1.1,

$$
U_{2}=-\frac{1}{2} \Re\left(\widetilde{\rho}_{1} U_{1}\right) / \rho_{0},
$$

which can be rewritten in terms of acoustic power to $U_{2}=-\dot{E}_{2} / p_{0}$. Assuming a regenerator of stacked screens, the resulting pressure gradient can be estimated using 


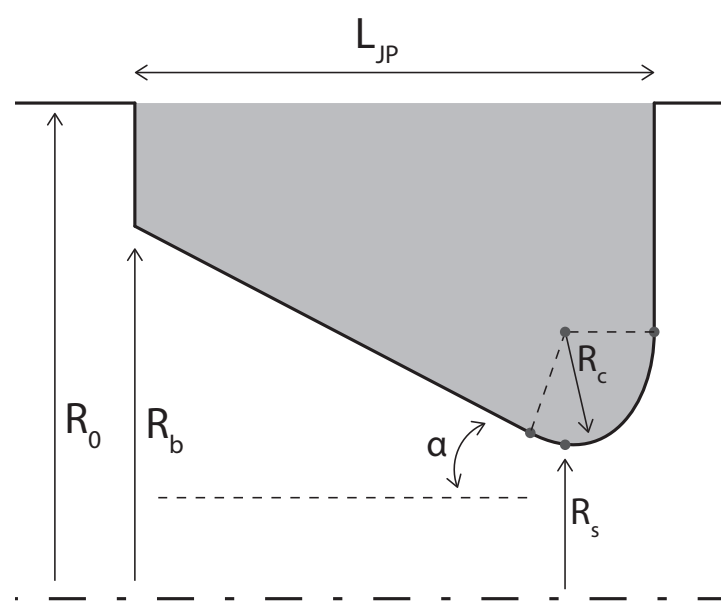

(a)

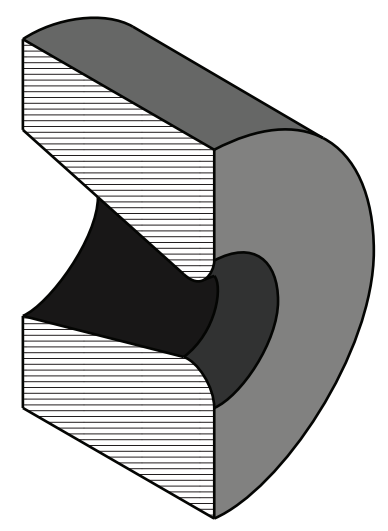

(b)

Figure 1.5: (a) Jet pump with parameters that define its geometry (not to scale). Bottom dashed line indicates center line, top solid line indicates outer tube wall. (b) Isometric representation of $\alpha=15^{\circ}$ jet pump sample.

theory presented by Swift et al. and Kays and London as ${ }^{6,53}$

$$
\Delta p_{2, r e g} \approx \frac{6}{A r_{h}^{2} p_{0}} \int_{0}^{L_{r e g}} \mu_{0}(x) \dot{E}_{2}(x) d x,
$$

with $A$ the cross-sectional area and $r_{h}$ the hydraulic diameter of the regenerator. The dynamic viscosity $\mu_{0}$ is spatially dependent due to the imposed temperature gradient across the regenerator. By balancing the time-averaged pressure drop across the jet pump with Eq. 1.4, Gedeon streaming can be suppressed.

A typical conical jet pump geometry is shown schematically in Fig. 1.5. The two openings both have a different radius: $R_{b}$ for the big opening (left side) and $R_{s}$ for the small opening. Due to the asymmetric geometry, a difference exists in the hydrodynamic end effects between the two flow directions in an oscillatory flow. These different hydrodynamic end effects result in a time-averaged pressure drop across the jet pump. Together with the jet pump length, $L_{J P}$, the jet pump taper angle $\alpha$ is defined. Furthermore, at the small opening a curvature $R_{c}$ is applied to further increase the asymmetry in the hydrodynamic end effects compared to a sharp contraction. A smooth contraction leads to less pressure loss in the leftward flow direction, therewith increasing the total time-averaged pressure drop.

Several jet pump geometries have been evaluated in literature. Swift et al. used a block with four tapered holes having a taper angle of $3.2^{\circ} .^{6}$ In the traveling wave thermoacoustic engine of Backhaus and Swift, an adjustable jet pump was used with two rectangular slits. ${ }^{7}$ The plate distance at the big opening was fixed whereas the small opening could be varied. A different jet pump geometry is examined by Biwa et al. by using a straight tube narrowing. ${ }^{54}$ The asymmetry in this case is generated by 
a difference in the tube termination only: at one side the narrow tube connects to a cone-shaped transition to the outer tube while at the other side the narrow tube protrudes into the outer tube.

All these jet pumps have a considerable length in order to have a "gentle" taper angle to avoid possible flow separation. This type of taper together with the required difference in cross-sectional areas (defined by radii $R_{b}$ and $R_{s}$ in Fig. 1.5) to obtain the desired time-averaged pressure drop results in a considerable segment size that has to fit inside the thermoacoustic device. To be able to manufacture compact thermoacoustic devices, a more compact jet pump design is desired. The size of a jet pump can be reduced by employing multiple parallel orifices, also referred to as a jet plate. This reduces the jet pump length while the total cross-sectional areas and the taper angle remain unchanged. One record of a jet plate with a large number of holes is the work of Haberbusch et al., where a total of 109 holes is used. ${ }^{34,55}$ Wilcox and Spoor have proposed a similar compact jet plate, which is designed in light of the current research project. ${ }^{56} \mathrm{~A}$ jet plate with 16 holes was used with a total length of less than $20 \mathrm{~mm}$.

\subsubsection{Quasi-steady approximation}

Despite the proven effectiveness of jet pumps, there is a lack of understanding with respect to the exact fluid dynamics that lead to the observed pressure drop. Current criteria for the design of a jet pump assume that the flow at any point in time has little "memory" of its past history, which is often referred to as the Iguchi-hypothesis. ${ }^{57}$ This allows the acoustic behavior to be based on a quasi-steady approximation using minor loss coefficients reported for steady pipe flow. ${ }^{6}$

The pressure drop generated by an abrupt pipe transition in steady flow can be calculated using

$$
\Delta p_{m l}=\frac{1}{2} K \rho u^{2},
$$

where $K$ is the minor loss coefficient which depends on geometry and flow direction, $\rho$ is the fluid density and $u$ is the fluid velocity. For an abrupt expansion, $K=K_{\exp }$ and can be estimated using the Borda-Carnot equation, ${ }^{58}$

$$
K_{\text {exp }}=\left(1-\frac{A_{s}}{A_{0}}\right)^{2},
$$

where $A_{s}$ is the cross-sectional area before the expansion and $A_{0}$ is the cross-sectional area right after the expansion. Note that these values assume a uniform velocity profile. Non-uniform velocity profiles will result in larger minor loss coefficient values for expansion. ${ }^{59}$

For a contraction, the steady flow minor loss coefficient is dependent upon the dimensionless curvature of the transition, $R_{c} / D$, where $R_{c}$ is the radius of curvature and $D$ is the diameter of the opening. ${ }^{58}$ For a sharp contraction (i.e. $R_{c} / D=0$ ), $K_{\text {con }}=0.5$ but this reduces to $K_{c o n}=0.04$ for $R_{c} / D \geq 0.15$. This reduced minor loss coefficient 
is the motivation for applying a smooth contraction at a jet pump's small opening to enhance the time-averaged pressure drop.

Under the assumption that the Iguchi-hypothesis is applicable and that the minor loss coefficients have the same values in oscillatory flow as they do in steady flow, a quasi-steady model has been formulated by Backhaus and Swift to calculate the time-averaged pressure drop across a jet pump, ${ }^{7}$

$$
\Delta p_{2, J P}=\frac{1}{8} \rho_{0}\left|u_{1, J P}\right|^{2}\left[\left(K_{e x p, s}-K_{c o n, s}\right)+\left(\frac{A_{s}}{A_{b}}\right)^{2}\left(K_{c o n, b}-K_{e x p, b}\right)\right],
$$

where $\left|u_{1, J P}\right|$ is the velocity amplitude at the small exit of the jet pump. The subscripts $s$ and $b$ indicate the small and big opening of the jet pump, respectively.

Although this time-averaged pressure drop can be exploited to cancel Gedeon streaming and improve the efficiency of a looped thermoacoustic device, this approach is not without penalty. Adding a jet pump results in additional dissipation of acoustic power. With the instantaneous acoustic power dissipation given by $\Delta p_{J P}(t) U_{J P}(t)$, and under the same previous assumptions, the time-averaged acoustic power dissipation across a jet pump is ${ }^{7}$

$$
\Delta \dot{E}_{J P}=\frac{\rho_{0}\left|u_{1, J P}\right|^{3} A_{s}}{3 \pi}\left[\left(K_{e x p, s}+K_{c o n, s}\right)+\left(\frac{A_{s}}{A_{b}}\right)^{2}\left(K_{c o n, b}+K_{e x p, b}\right)\right] .
$$

An optimal jet pump should establish the required amount of time-averaged pressure drop to cancel any Gedeon streaming with minimal acoustic power dissipation. According to the quasi-steady approximation, this requires maximizing the difference in minor losses due to contraction and expansion while at the same time minimizing the sum of the minor loss coefficients. Designs such as the one presented in Fig. 1.5 have been based on this analysis.

Qualitative evidence exists which supports the current analysis, but quantitative agreement between the theory and experiments remains poor. ${ }^{7,60}$ While the accuracy of this approach is yet unknown, it is assumed valid for large displacement amplitudes in relation to the jet pump dimensions. ${ }^{7}$ Moreover, when using minor loss coefficients for steady expansion and contraction, the effect of the jet pump taper angle or the jet pump length is not included in the current theory while it is observed to have an important effect on the jet pump pressure drop. ${ }^{60,61}$

\subsubsection{Literature review}

Previous studies related to jet pumps for thermoacoustic applications include mainly applied work; only a few studies on the actual flow physics have been published to date. Petculescu and Wilen measured the pressure drop for a series of jet pump geometries in a standing wave experimental apparatus. ${ }^{60}$ They then derived minor loss coefficients based on the measured pressure and the velocity in the jet pump waist, which was estimated using an acoustic network model. A difference between 
the measured and theoretical minor loss coefficients is reported, especially for the diverging flow direction. However, for the investigated geometries - up to a taper angle of $10^{\circ}$ - good agreement between the performed steady flow and oscillating flow experiments is obtained. An increase in the taper angle is shown to have a negative effect on the time-averaged pressure drop.

Although studies on jet pump flow physics are scarce, parallels can be drawn between the flow in jet pumps and that in several other geometries. The flow from the small to the big opening shows similarities with diffusers. ${ }^{62,63}$ Certain flow features occurring when the flow exits from the small jet pump opening, such as the aforementioned vortex rings, are also observed from oscillatory flows in nozzles, abrupt expansions, orifices and synthetic jet applications. ${ }^{5,64-68}$

Smith and Swift have experimentally studied oscillatory flow through a nozzle with constant diameter, simulating one end of a jet pump. ${ }^{64}$ In their work, a nozzle is connected to open space, establishing a non-confined jet. A parametric study on the time-averaged pressure drop and the acoustic power dissipation is performed, identifying some of the dimensionless quantities which describe the flow phenomena: the dimensionless stroke length, the dimensionless curvature and the acoustic Reynolds number. It is concluded that "extensive numerical studies" are required for a further understanding of the minor loss phenomena to control streaming.

Computational studies related to jet pumps mainly include the work of Boluriaan and Morris. ${ }^{69,70}$ In two studies, the minor losses due to a single diameter transition under standing wave conditions are simulated using a two-dimensional computational fluid dynamics (CFD) model. The standing wave is generated by either applying an oscillatory body force ("shaking" the domain) or by using an oscillatory line source inside the domain. Axial pressure and velocity profiles are presented and the effect of jetting and vortex shedding on the flow field is described. The time-averaged pressure drop across the transition is found to be a factor of three higher than the quasi-steady solution. In a separate study, a jet pump geometry is investigated using a similar CFD model. ${ }^{71}$ In this case, a combination of two line sources with a non-reflecting boundary condition on either side is used to generate a traveling wave inside the domain. The flow field is calculated for a single jet pump geometry and wave amplitude. More recently, Tang et al. investigated the performance of jet pumps numerically, ${ }^{72}$ but assumed a priori the quasi-steady approximation to be valid by modeling the flow as two separate steady flows. The negative effect of flow separation on the jet pump performance was identified which is in line with the current work.

\subsection{Thesis outline}

The current research focuses on predicting the jet pump performance as a function of its geometry and relating this to flow features occurring. The quasi-steady approximation introduced in Section 1.3.1 serves as a starting point, but upon reducing the jet pump length the assumptions on which the theory is based are void.

Five different studies have been carried out and are presented as separate chapters 
in this thesis. In Chapter 2, a computational fluid dynamics model of a conical jet pump is introduced. Using this model, different flow regimes are identified and linked to the jet pump performance in terms of its time-averaged pressure drop and acoustic power dissipation. The CFD model is further used in Chapter 3 for a geometric parameter study to investigate how the jet pump taper angle, small opening size, and edge curvature affect the flow field and performance.

From these two studies, flow separation inside the jet pump at high taper angles or large wave amplitudes is identified as a major issue that has a negative effect on the jet pump effectiveness. Chapter 4 focuses on further characterizing the flow separation using the aforementioned CFD model. A design alteration is proposed that can avoid the occurrence of flow separation and results in a more robust and effective jet pump. The flow separation is further identified experimentally in Chapter 5 with a focus on turbulent oscillatory flows. Hot-wire anemometry is used to detect the onset of flow separation and the level of turbulence.

Where the work in Chapters $2-5$ discusses jet pumps with a single tapered hole, the behavior of using multiple parallel orifices is investigated in Chapter 6. Different jet pump samples having 1 to 16 holes are used and the time-averaged pressure drop and acoustic power dissipation is measured experimentally. The contribution of the hole-to-hole spacing versus the miniaturization of the individual holes is separated. Flow visualization using smoke particles and high-speed image acquisition allows to study the interaction of adjacent vortex rings.

Chapter 7 summarizes the conclusions from the individual chapters and aims to provide an overlook of the current work, as well as an outlook for future research. 


\section{Numerical investigation on the jet pump flow regimes in laminar oscillatory flows}

A two-dimensional computational fluid dynamics model is used to predict the oscillatory flow through a tapered cylindrical tube section (jet pump) placed in a larger outer tube. The performance of two jet pump geometries with different taper angles is investigated. A specific time-domain impedance boundary condition is implemented in order to simulate traveling acoustic wave conditions. It is shown that by scaling the acoustic displacement amplitude to the jet pump dimensions, similar minor losses are observed independent of the jet pump geometry. Four different flow regimes are distinguished and the observed flow phenomena are related to the jet pump performance. The simulated jet pump performance is compared to an existing quasi-steady approximation which is shown to only be valid for small displacement amplitudes compared to the jet pump length.

\subsection{Introduction}

In this chapter, the oscillatory flow in the vicinity of a jet pump is investigated using a CFD model which is described in Section 2.2. Using this CFD model, the performance of two jet pump geometries with different taper angles are studied. Four different flow regimes are described (Section 2.3.1) and subsequently linked to the observed jet pump performance. The time-averaged pressure drop and acoustic power dissipation are scaled to relate the behavior of the two different taper angles and a comparison with the quasi-steady approximation is made (Section 2.3.3).

The precursor of the study presented in this chapter is a preliminary study, ${ }^{61}$ where the effect of the jet pump taper angle on the time-averaged pressure drop is inves-

Adapted from J. P. Oosterhuis, S. Bühler, D. Wilcox, and T. H. van der Meer. "A numerical investigation on the vortex formation and flow separation of the oscillatory flow in jet pumps." $J$. Acoust. Soc. Am., 137(4):1722-1731, 2015. doi:10.1121/1.4916279 


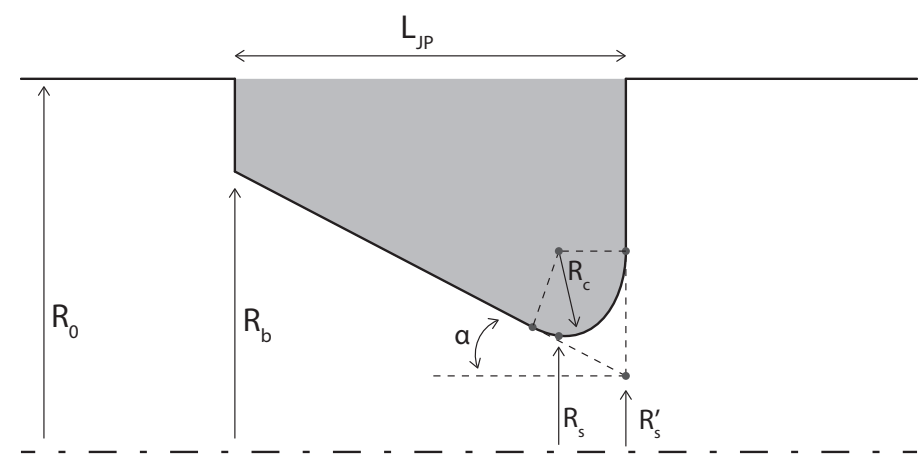

Figure 2.1: Jet pump with parameters that define the geometry (not to scale). Virtual point $R_{s}^{\prime}$ represents small radius without curvature and is used for jet pump length calculation. Bottom dashed line indicates centerline, top solid line indicates tube wall.

tigated numerically and compared against the experimental work of Petculescu and Wilen. ${ }^{60}$ A clear decrease in time-averaged pressure drop is observed at higher taper angles, which is one of the motivations for the work presented here.

\subsection{Modeling}

An axisymmetric CFD model is developed using the commercial software package ANSYS CFX version 14.5, ${ }^{74}$ which has been used successfully in the simulation of various (thermo)acoustic applications. ${ }^{75-77}$ The jet pump is placed in an outer tube to study the influence of the jet pump geometry on the flow field. Boundary conditions are applied to simulate a traveling wave inside the computational domain; these are discussed in Sections 2.2.2 and 2.2.3. In all cases, air at a mean temperature of $T_{0}=300 \mathrm{~K}$ and a mean pressure of $p_{0}=1 \mathrm{~atm}$ is used as the working fluid. Three different driving frequencies are investigated: $50 \mathrm{~Hz}, 100 \mathrm{~Hz}$ and $200 \mathrm{~Hz}$.

\subsubsection{Geometry}

The jet pump geometry is shown in Fig. 2.1 and is defined using a reduced number of parameters: the radius of the big exit $R_{b}$, the effective radius of the small exit (the jet pump "waist") $R_{s}$, the taper half-angle $\alpha$ and the radius of curvature at the small exit of the jet pump $R_{c}$. Based on these parameters, the other parameters can be calculated. The total jet pump length $L_{J P}$ is

$$
L_{J P}=\frac{R_{b}-R_{s}^{\prime}}{\tan \alpha}
$$

where $R_{s}^{\prime}$ is the small radius of the jet pump without any curvature applied,

$$
R_{s}^{\prime}=R_{s}-R_{c}\left(\frac{\sin \alpha+1}{\cos \alpha}-1\right) .
$$


Table 2.1: Jet pump length $L_{J P}$ for applied taper angles $\alpha$.
Table 2.2: Dimensions of simulated jet pump geometries.

\begin{tabular}{ll}
\hline$\alpha$ & $L_{J P}$ \\
\hline $7^{\circ}$ & $70.5 \mathrm{~mm}$ \\
$15^{\circ}$ & $35.5 \mathrm{~mm}$ \\
\hline
\end{tabular}

\begin{tabular}{ll}
\hline$R_{0}$ & $30 \mathrm{~mm}$ \\
$R_{b}$ & $15 \mathrm{~mm}$ \\
$R_{s}$ & $7 \mathrm{~mm}$ \\
$R_{c}$ & $5 \mathrm{~mm}$ \\
\hline
\end{tabular}

In addition to the jet pump region, the computational domain consists of a section of the outer tube on both sides of the jet pump with a radius of $R_{0}=30 \mathrm{~mm}$ and a length of $L_{0}=500 \mathrm{~mm}$ each for the cases where $f=100 \mathrm{~Hz}$. The influence of the length of this section on the jet pump performance and vortex propagation characteristics has been verified by comparing with results from simulations using $L_{0}=100 \mathrm{~mm}$ and no significant difference was observed. Although a shorter outer tube section will lead to a reduced computational time, the longer length is used in order to study the resulting flow field on both sides of the jet pump in detail. For the other two driving frequencies $(50 \mathrm{~Hz}$ and $200 \mathrm{~Hz}), L_{0}$ is scaled relative to the acoustic wavelength to avoid the jet pump being placed at a velocity node.

Two different taper angles, $7^{\circ}$ and $15^{\circ}$, are analyzed by changing the jet pump length. The corresponding jet pump lengths are shown in Table 2.1. All the other geometrical parameters remain the same and are listed in Table 2.2. The dimensionless curvature is $R_{c} / D_{s}=0.36$ for both geometries, which is well above the limit for a "smooth" contraction. Hence, according to steady flow literature, ${ }^{58} K_{\text {con }}=0.04$. The ratio between the small and big cross-sectional area is $R_{s}^{2} / R_{b}^{2}=0.22$ for both geometries. Because the cross-sectional area of both jet pump openings is kept constant, one would expect an identical pressure drop and acoustic power dissipation based on the quasisteady approximation (Sec. 1.3.1). Moreover, the term $\left(A_{s} / A_{b}\right)^{2}$ is small such that the minor losses due to the small opening of the jet pump are expected to predominantly determine the time-averaged pressure drop and acoustic power dissipation.

\subsubsection{Numerical setup}

Within the described computational domain, the unsteady, fully compressible NavierStokes equations are solved. The ideal gas law is used as an equation of state whereas the energy transport is described using the total energy equation including viscous work terms. ${ }^{78}$ No additional turbulence modeling is applied as all presented results fall within the laminar regime (see Section 2.3.3). The governing equations are discretized in space using a high resolution advection scheme and discretized in time using a second order backward Euler scheme. Each wave period is discretized using 1000 time-steps which yields a time-step size of $\Delta t=1 \cdot 10^{-5} \mathrm{~s}$ for $f=100 \mathrm{~Hz}$. For each simulation case, a total of $N_{p}=10$ wave periods are simulated. With a typical computational mesh, the total single core computational time is about 40 hours on an Intel Core i7 CPU. 


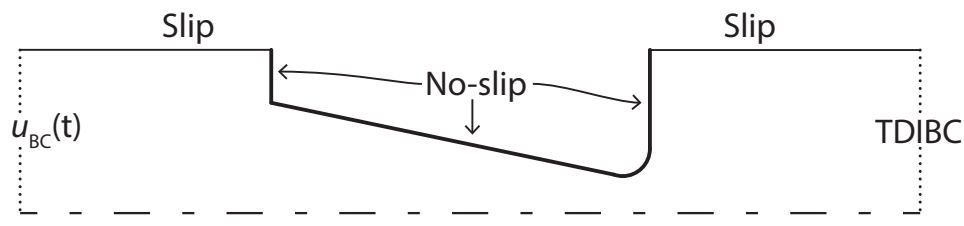

Figure 2.2: Schematic representation of numerical setup with boundary conditions.

In order to perform an axisymmetric simulation in ANSYS CFX, a computational mesh which extends one element in the azimuthal direction is required and symmetry boundary conditions are applied on the originating faces normal to the azimuthal direction. On the radial boundary of the outer tube (at $r=R_{0}$ ), a slip adiabatic wall boundary condition is used as the pipe losses in this part of the domain are currently not of interest. To correctly simulate the minor losses in the jet pump, a no-slip adiabatic wall boundary condition is used at the walls of the jet pump. The used boundary conditions are shown schematically in Fig. 2.2 .

To generate an acoustic wave, a velocity boundary condition is used at $x=0$ which oscillates in time according to $u(t)=u_{1} \sin (2 \pi f t)$ with $u_{1}$ a defined velocity amplitude. On the right boundary of the computational domain, at $x=L$, a dedicated time-domain impedance boundary condition is applied with a specified reflection coefficient of $|\mathcal{R}|=0$, as described in Section 2.2.3. This ensures free propagation of the acoustic wave without any additional reflections being introduced into the computational domain. ${ }^{79}$ The combination of the velocity boundary condition and the time-domain impedance boundary condition results in a time-averaged volume flow that is on average less than $0.5 \%$ of the acoustic volume flow rate.

\subsubsection{Time-domain impedance boundary condition}

A specific time-domain impedance boundary condition has been implemented in ANSYS CFX based on the work of Polifke et al. ${ }^{80,81}$ This approach ultimately defines the pressure $p(t)$ on the boundary. The applied pressure is based on wave information from inside the domain at a previous time-step which is sampled at a distance $\Delta x=50 \mathrm{~mm}$ from the boundary. Moreover, an external perturbation can be introduced on the boundary such that any complex reflection coefficient can be specified. However, in this study only a non-reflective situation $(\mathcal{R} \mid=0)$ is considered. The measured reflection coefficient typically ranges from $1 \%$ to $2 \%$. Further details about the exact implementation and validation can be found in the work of Van der Poel which has been carried out as part of the current research. ${ }^{79}$ Additionally, the simulated flow field in a tube without a jet pump is compared with an analytic wave propagation model ${ }^{82}$ and excellent agreement is obtained. An overview of the performed validation study is given in Appendix A. 


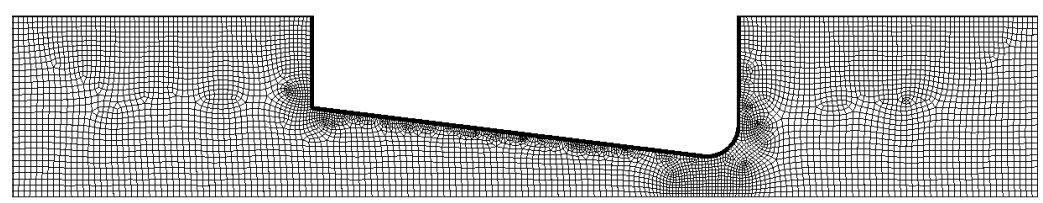

Figure 2.3: Computational mesh, close-up near jet pump.

\subsubsection{Data analysis}

From the CFD results, a transient solution field for all flow variables is obtained. In order to obtain the complex amplitudes (denoted with the subscript "1"), a pointwise discrete Fourier term is calculated for the specified wave frequency using data from the last five simulated wave periods. The jet pump velocity amplitude $\left|u_{1, J P}\right|$ is calculated using an area-weighted average of the velocity amplitude at the local grid points in the smallest opening of the jet pump (see Fig. 2.1). The time-averaged variables (denoted with the subscript "2") are calculated by averaging the time-series solution over an integer number of wave periods, thus eliminating all first order effects. Note that for the time-averaged streaming velocity field a density-weighted average is applied,

$$
\mathbf{u}_{2}=\frac{\langle\rho \mathbf{u}\rangle}{\langle\rho\rangle}
$$

where $\langle\ldots\rangle$ indicates time-averaging.

\subsubsection{Computational mesh}

The resolution of the computational mesh is defined based on the maximum element size in various regions of the domain. A maximum element size inside the jet pump region of $1 \mathrm{~mm}$ is used, which is refined up to a maximum size of $0.5 \mathrm{~mm}$ near the jet pump waist as is visible in Fig. 2.3. Moreover, to be able to accurately resolve the flow separation, a refinement in the viscous boundary layer is applied such that a minimum of 10 elements reside within one viscous penetration depth distance $\delta_{\nu}=\sqrt{2 \mu / \omega \rho}$ from the jet pump wall. At a distance of $50 \mathrm{~mm}$ away from the jet pump, a transition to a structured mesh is applied to allow for uncoupling of the gradients in the $x$ and $r$ directions and consequently for the use of a large aspect ratio as the gradients far away from the jet pump are much larger in the radial direction than they are in the axial direction. The axial element size grows towards the axial boundaries up to a maximum element size of $10 \mathrm{~mm}$ which is sufficient for solving the acoustic wave propagation. In the radial direction, a maximum element size of $1 \mathrm{~mm}$ is used throughout the outer tube. For the current geometry, this yields a total mesh size of 36236 nodes.

In order to validate the computational mesh, the results of three different mesh resolutions are compared based on several key outcome quantities: the dimensionless time-averaged pressure drop $\left(\Delta p_{2}^{*}\right.$, Eq. 2.10), the dimensionless acoustic power dissipation $\left(\Delta \dot{E}_{2}^{*}\right.$, Eq. 2.11) and the propagation distance of the vortex street after ten 
Table 2.3: Specified element sizes for three different meshes used for validation purposes. For all other results presented, the medium mesh is used.

\begin{tabular}{lllll}
\hline \multirow{2}{*}{ Mesh } & \multicolumn{4}{c}{ max. element size } \\
& $N_{e l, B L}$ & jet pump jet pump & outer tube \\
& region & waist & \\
\hline Coarse & 5 & $2 \mathrm{~mm}$ & $1 \mathrm{~mm}$ & $10 \mathrm{~mm}$ \\
Medium & 10 & $1 \mathrm{~mm}$ & $0.5 \mathrm{~mm}$ & $10 \mathrm{~mm}$ \\
Fine & 20 & $0.5 \mathrm{~mm}$ & $0.25 \mathrm{~mm}$ & $10 \mathrm{~mm}$ \\
\hline
\end{tabular}

Table 2.4: Results of mesh validation study for a jet pump geometry having a taper angle of $\alpha=7^{\circ}$. The jet pump waist velocity is $\left|u_{1, J P}\right|=15.3 \mathrm{~m} / \mathrm{s}$, the driving frequency is $f=100 \mathrm{~Hz}$.

\begin{tabular}{lllll}
\hline Mesh & $N_{\text {nodes }}$ & $\Delta p_{2}^{*}$ & $\Delta \dot{E}_{2}^{*}$ & $\ell_{p}$ \\
\hline Coarse & 18442 & 0.40 & 0.58 & $0.81 \mathrm{~m}$ \\
Medium & 36236 & 0.84 & 0.86 & $0.92 \mathrm{~m}$ \\
Fine & 84618 & 0.86 & 0.97 & $0.96 \mathrm{~m}$ \\
\hline
\end{tabular}

wave periods, $\ell_{p}$. The specified element sizes for the three different meshes are listed in Table 2.3. In Table. 2.4 the total number of nodes is shown. They increase by approximately a factor of two between the subsequent mesh refinements.

Table. 2.4 shows the results for the three different meshes using an intermediate wave amplitude $\left(u_{1}=0.8 \mathrm{~m} / \mathrm{s}\right.$ which yields $\left.\left|u_{1, J P}\right|=15.3 \mathrm{~m} / \mathrm{s}\right)$ and a $7^{\circ}$ taper angle geometry, which is representative for the other simulated cases. The driving frequency is set to $100 \mathrm{~Hz}$. A clear deviation is visible for all outcome quantities between the coarse mesh and the other two meshes while the results between the medium and fine mesh are comparable. The dimensionless pressure drop and acoustic power dissipation obtained with the medium mesh, show a difference of $2.4 \%$ and $11.1 \%$ with the fine mesh, respectively. The vortex propagation distance deviates $3.5 \%$ with respect to the fine mesh. Hence, it was decided to use the medium mesh resolution for all future simulations.

\subsection{Results and discussion}

The described computational model has been used to investigate a range of wave amplitudes with the two described jet pump geometries $\left(\alpha=7^{\circ}\right.$ and $\left.15^{\circ}\right)$. It will be shown that the jet pump performance can be scaled based on the acoustic displacement amplitude with respect to the jet pump dimensions. Defining the acoustic displacement amplitude in the jet pump waist under the assumption of a sinusoidal jet pump velocity as

$$
\xi_{1, J P}=\frac{\left|u_{1, J P}\right|}{2 \pi f}
$$


the two Keulegan-Carpenter numbers can be defined based on the jet pump length $L_{J P}$ and waist diameter $D_{s}$, respectively,

$$
\begin{aligned}
K C_{L} & =\frac{\xi_{1, J P}}{L_{J P}}, \\
K C_{D} & =\frac{\xi_{1, J P}}{D_{s}} .
\end{aligned}
$$

Similar Keulegan-Carpenter numbers are used by Aben in the identification of the oscillatory flow behind a thermoacoustic stack. ${ }^{75}$ Following Smith and Swift, ${ }^{64} K C_{D}$ is similar to the dimensionless stroke length $L_{0} / h$ and can be rewritten to $R e_{D} / S^{2}$ where $R e_{D}$ is an acoustic Reynolds number based on diameter and $S=\sqrt{\omega D^{2} / \nu}$ the Stokes number. ${ }^{62,64,68} K C_{L}$ is one of the suggested additional dimensionless parameters that may affect the results. By investigating two jet pumps of different lengths, it will be shown that $K C_{L}$ is of high relevance to scale the jet pump performance properly.

Different observed flow regimes will be distinguished and the corresponding flow fields will be described in Section 2.3.1. Typical axial profiles of pressure, velocity and acoustic power will be described and used to define the jet pump performance. Finally, the time-averaged pressure drop and acoustic power dissipation will be scaled and shown as a function of the two Keulegan-Carpenter numbers in Section 2.3.3. In this way, the jet pump performance will be related to the observed flow phenomena and includes the influence of the jet pump taper angle.

\subsubsection{Flow regimes}

Independent of the jet pump geometry or frequency, four different flow regimes can be distinguished. Examples of these flow regimes are shown in Fig. 2.4 for the $7^{\circ}$ taper angle jet pump and a driving frequency of $f=100 \mathrm{~Hz}$. The top graph of each figure shows the instantaneous vorticity field at the last simulated time-step $t_{\max }=0.1 \mathrm{~s}$. The centers of the propagating vortex rings can be identified as local maxima in the instantaneous vorticity field. The bottom graph of each figure shows the timeaveraged velocity field $u_{2}$. The black line denotes the location of zero streaming velocity. Figure 2.5 shows the axial velocity over the radius inside the jet pump. The different lines are separated $\varphi=\pi / 2$ in time. Each figure represents a different flow regime which corresponds to the flow regimes shown in Fig. 2.4.

In all simulated cases, a vortex pair is formed on either side of the jet pump. However, for low amplitudes the vortex pairs are not shed and merely oscillate locally with the acoustic field. This results in a zero time-averaged pressure drop and negligible acoustic power dissipation. An example of this flow regime is shown in Fig. 2.4a where on either side of the jet pump a small vortex can be observed. The corresponding velocity profiles inside the jet pump are shown in Fig. 2.5a, the profiles are identical but opposite during the forward and backward flow direction representing a pure harmonic oscillation. The influence of the viscous boundary layer is visible but further away from the boundary a constant velocity is observed. 


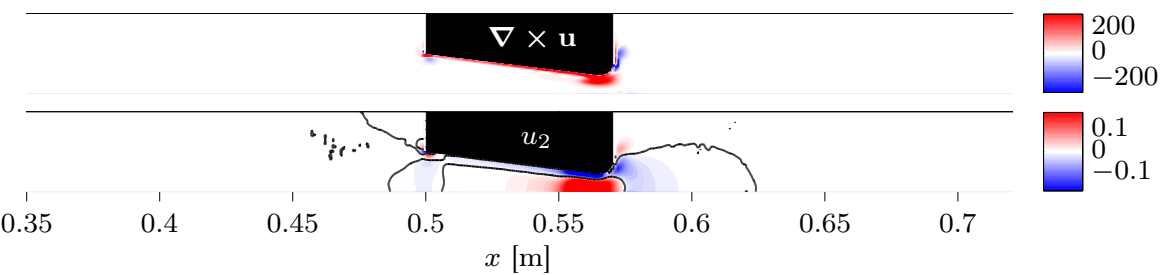

(a) Oscillatory vortex pair on both sides, no jetting observed. $K C_{L}=0.09$, $K C_{D}=0.46, \Delta p_{2}^{*}=0.04$.

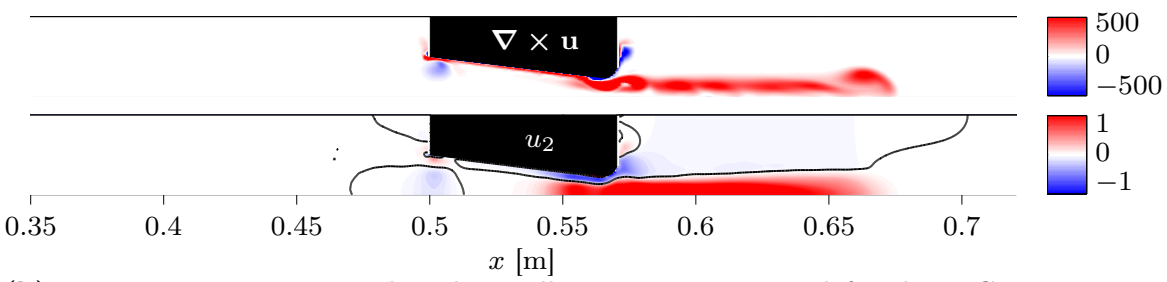

(b) Propagating vortex to right side, oscillating vortex pair on left side. $K C_{L}=0.18$, $K C_{D}=0.92, \Delta p_{2}^{*}=0.40$.

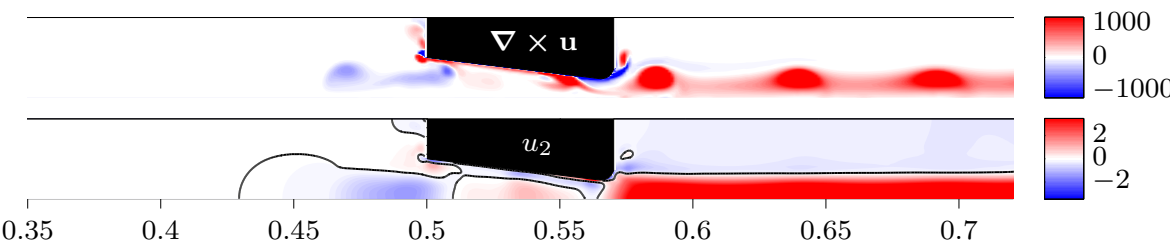

$x[\mathrm{~m}]$

(c) Propagating vortex on both sides. $K C_{L}=0.35, K C_{D}=1.74, \Delta p_{2}^{*}=0.84$.

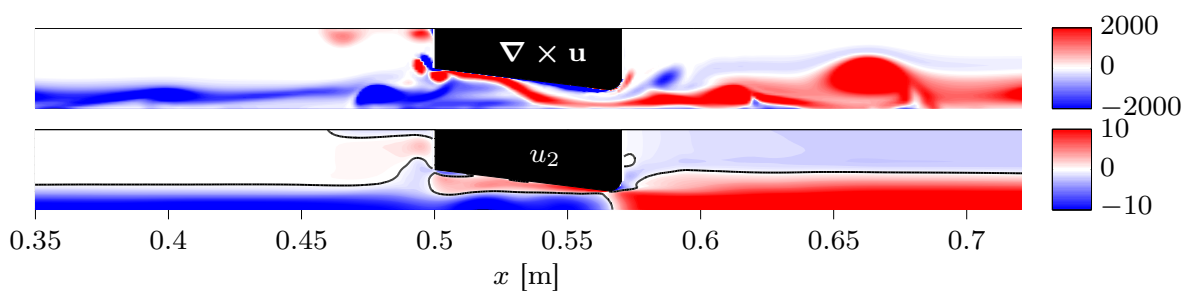

(d) Left propagating vortex from waist of jet pump, flow separation inside the jet pump occurs. $K C_{L}=1.17, K C_{D}=5.90, \Delta p_{2}^{*}=0.46$.

Figure 2.4: Four different flow regimes are distinguished based on the Keulegan-Carpenter numbers $K C_{L}$ and $K C_{D}$ using the instantaneous vorticity fields $\nabla \times \mathbf{u}[1 / \mathrm{s}]$ at $t=t_{\max }$ (top) and streaming velocity fields $u_{2}[\mathrm{~m} / \mathrm{s}]$ (bottom) around the jet pump for the $\alpha=7^{\circ}$ geometry, $f=100 \mathrm{~Hz}$. Black line in streaming velocity field indicates transition from positive to negative velocity. 


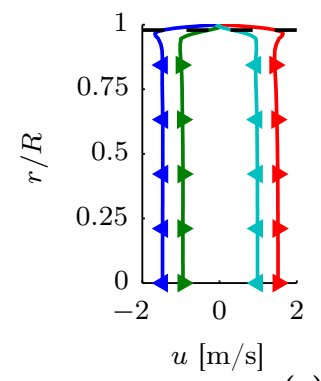

(a)

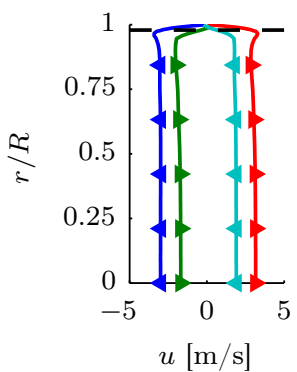

(b)

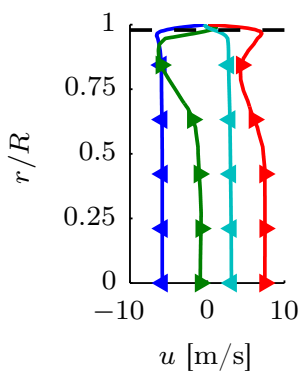

(c)

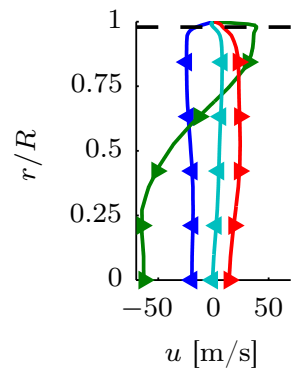

(d)

Figure 2.5: Axial velocity $u$ over the radius at four time instances during the last wave period $\left(\varphi=0^{\circ}, 90^{\circ}, 180^{\circ}\right.$ and $\left.270^{\circ}\right)$ halfway inside the jet pump $\left(x=L_{0}+L_{J P} / 2\right)$. Figures (a) $-(\mathbf{d})$ correspond to the four different flow regimes distinguished in Fig. 2.4. Arrows indicate acceleration and deceleration: - for $\partial u / \partial t>0$ and $\varangle$ for $\partial u / \partial t<0 . r / R=1$ denotes the jet pump wall and $r / R=0$ is the centerline location. The dashed line indicates the thickness of the viscous boundary layer $\left(\delta_{\nu} / R\right)$ at the jet pump wall.

If the displacement amplitude is larger than the radius of one of the jet pump openings, the vortex pair on the corresponding side is shed and propagation starts. Hence, for $K C_{D}>0.5$ vortex propagation on the right side of the jet pump can be observed. An example of this flow regime is shown in Fig. 2.4b where $K C_{D}=0.92$. In the streaming velocity field (bottom graph), a steady jet in the positive $x$-direction can be observed. The occurrence of vortex shedding corresponds well to an increase in the time-averaged pressure drop. The velocity profiles inside the jet pump are shown in Fig. 2.5b and are comparable to the first flow regime where a harmonic oscillation is observed.

Figure 2.4c shows an intermediate flow regime where vortex propagation to the left side of the jet pump can be observed in addition to the right-sided propagation. However, the flow field is still rather asymmetric on both sides of the jet pump which results in a high time-averaged pressure drop. The vortex propagation speed $u_{v}$ is strongly dependent on the velocity amplitude in the jet pump waist. Comparing this flow regime to the previous, the wave amplitude and correspondingly the vortex propagation speed has increased. This results in vortices clearly separated from each other. In the streaming velocity field, a recirculation zone inside the jet pump can be observed which is caused by a difference in the velocity profile during the accelerating and decelerating phase. Figure $2.5 \mathrm{c}$ shows the velocity profile inside the jet pump at four different time instances. When the fluid is accelerating $(\partial u / \partial t>0$, indicated

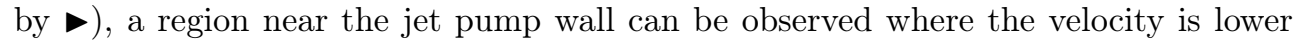
compared to the bulk velocity, regardless of the direction of the bulk flow. This is initiated when the negative bulk velocity is at its maximum and the acceleration changes sign. During the remainder of the acceleration phase, the velocity near the jet pump wall (but outside the viscous boundary layer) "lags" the bulk flow. When the fluid starts decelerating (indicated by ४), a pure acoustic velocity profile is again observed. This difference in velocity profiles leads to a time-averaged recirculation inside the jet pump. 
The fourth flow regime is observed when the displacement amplitude is larger than the jet pump length $\left(K C_{L}>1\right)$. An example of this case is shown in Fig. 2.4d. Vortices are now displaced from the right jet pump tip through the jet pump to the left, resulting in an additional steady jet in the negative $x$-direction. During the other half of the acoustic period, vortices shed from the left jet pump tip are displaced through the jet pump to the right and propagate in the positive $x$-direction, contributing to the existing steady jet on this side of the jet pump. These vortex rings are smaller and propagate with a lower speed because they are shed at a location where the velocity amplitude is lower than in the jet pump waist. After some distance (outside the shown region), the smaller rings merge with the larger vortex rings originating from the jet pump waist.

The existing jet through the jet pump causes time-averaged flow separation inside the jet pump which is visible in the bottom graph of Fig. 2.4d. In contrast with the previous flow regimes, now a positive streaming velocity exists close to the jet pump wall. Examining the instantaneous velocity profiles inside the jet pump in Fig. 2.5d gives more insight into the flow separation process. When the flow starts accelerating during the backward flow phase, the local shear stress at the jet pump wall, $\left.\mu \frac{\partial u}{\partial r}\right|_{r=R}$ becomes zero and the flow separation is initiated (line marked with - and negative centerline velocity). After the flow reversal, the flow becomes uni-directional again for the remainder of the wave period. This is independent of the sign of $\partial u / \partial t$. Although the radius of curvature meets the steady flow criterion for a "smooth" contraction $\left(R_{c} / D_{s}=0.36>0.15\right)$, it is expected that the curvature plays an important role in the flow separation process. ${ }^{66}$ This is further investigated in Chapter 3.

\subsubsection{Axial profiles}

Before describing the relation between the jet pump performance and the jet pump waist velocity, the results for a typical simulation case are described for the $7^{\circ}$ taper angle jet pump geometry with a driving frequency of $f=100 \mathrm{~Hz}$. These results correspond to the flow field shown in Fig. 2.4c where a steady jet to the right side of the jet pump exists and vortex propagation to the left side of the jet pump has just started. On the left boundary condition, a far field velocity amplitude of $u_{1}=$ $0.8 \mathrm{~m} / \mathrm{s}$ is specified resulting in a velocity amplitude in the jet pump waist of $\left|u_{1, J P}\right|=$ $15.3 \mathrm{~m} / \mathrm{s}$.

Figure 2.6a shows the velocity amplitude and the time-averaged velocity profile along the $x$-axis. The area-averaged velocity amplitude (dashed gray line) shows nearly incompressible behavior where the velocity is inversely proportional to the crosssectional area. The volume flow rate $U_{1}$ to the right of the jet pump is constant to within $0.7 \%$. The irregularities in the velocity amplitude at the centerline (solid gray line) to the right of the jet pump are caused by the vortex shedding. A positive timeaveraged centerline velocity (solid black line) to the right of the jet pump indicates a steady jet being formed from the jet opening. Note that the jet has only propagated a distance of $\ell_{p}=0.35 \mathrm{~m}$ from the jet pump. Furthermore, the steady jet is balanced 

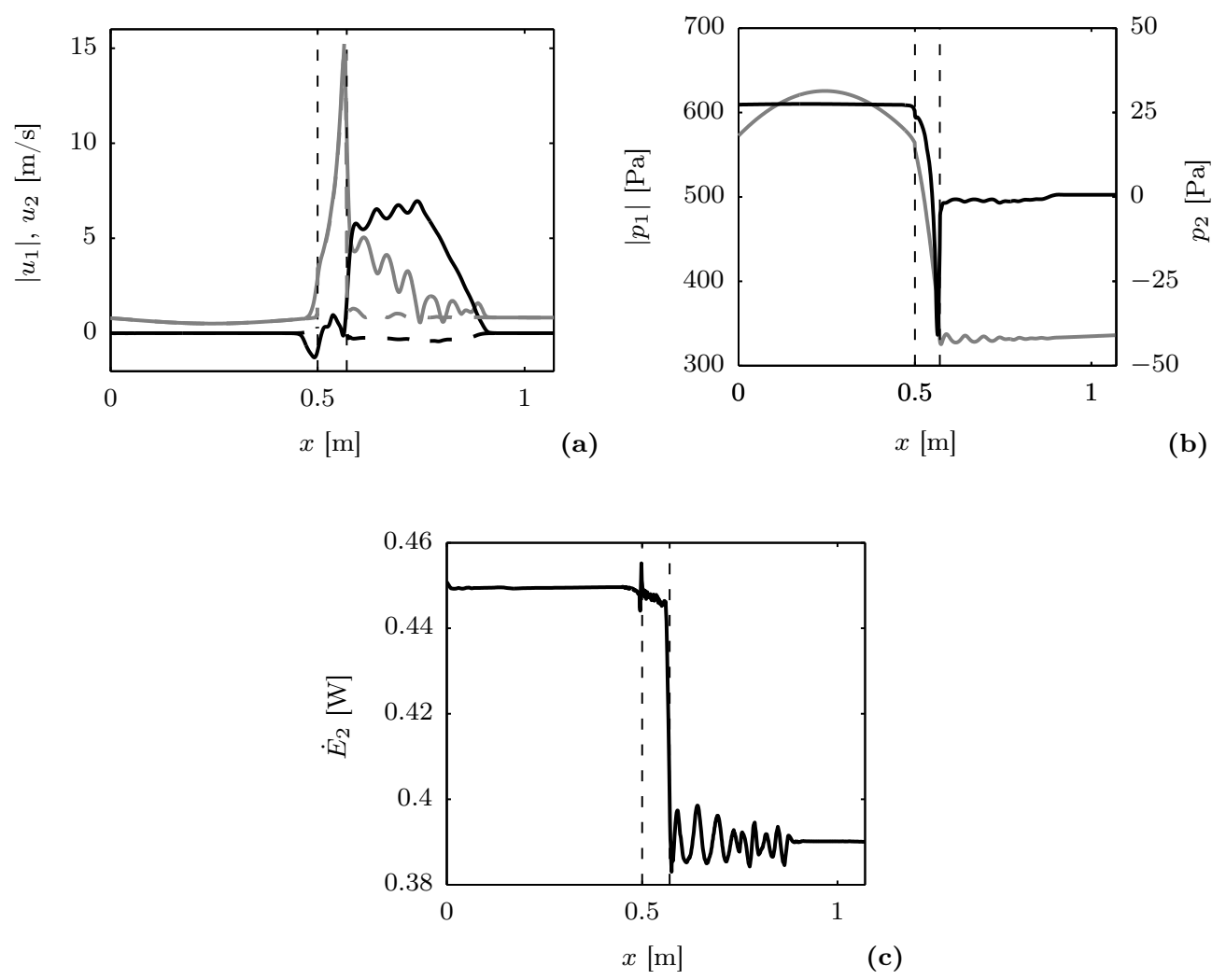

Figure 2.6: (a) Velocity amplitude $\left|u_{1}\right|$ at $r=0$ (gray, solid) and area-averaged over the cross-section (gray, dashed). Black lines show streaming velocity $u_{2}$ at $r=0$ (solid) and at $r=\frac{2}{3} R_{0}$ (dashed). (b) Area-averaged pressure amplitude $\left|p_{1}\right|$ (gray, left axis) and time-averaged pressure $p_{2}$ (black, right axis). (c) Acoustic power $\dot{E}_{2}$. All plotted along $x$-axis using a jet pump taper angle of $\alpha=7^{\circ}$. Jet pump waist velocity amplitude is $\left|u_{1, J P}\right|=15.3 \mathrm{~m} / \mathrm{s}$ and $f=100 \mathrm{~Hz}$, corresponding to the flow fields in Fig. 2.4c. Vertical dashed lines indicate the exits of the jet pump. 
by a mean flow in the opposite direction closer to the tube wall (dashed black line) to ensure a zero mean mass flux over the cross-section.

Figure 2.6b shows both the pressure amplitude (gray line, left axis) and the timeaveraged pressure (black line, right axis). The constant pressure amplitude to the right of the jet pump indicates that a traveling wave exists in this part of the domain. On the left side of the jet pump a standing wave component is present due to reflection of the acoustic wave off the jet pump surface. This is confirmed by calculating the reflection coefficient. On the right side $\left|\mathscr{R}_{R}\right|=1.16 \%$ while on the left side $\left|\mathscr{R}_{L}\right|=$ $49.9 \%$. The time-averaged pressure in Fig. 2.6b shows a clear drop between left and right of the jet pump which is one of the main measures of performance of the jet pump. While one could simply subtract the time-averaged pressure $p_{2}$ at two locations on either side of the jet pump, this would lead to inconsistent results due to the influence of vortex shedding on the time-averaged pressure profile. This is visible in Fig. 2.6b and becomes more dominant at higher wave amplitudes. Because the vortex propagation speed is much lower than the speed of sound, time-averaging over a multiple of the acoustic period does not remove the contribution of the vortex propagation to the pressure field. Alternatively, a spatially average of $p_{2}(x)$ on either side of the jet pump is calculated with specific averaging intervals starting at a distance of $2 \cdot \xi_{1, J P}$ from the jet pump up to the total vortex propagation distance $\ell_{p}$. When no vortex street is present on the corresponding side of the jet pump, the averaging is carried out up to the axial extremities of the domain. The two resulting spatial averages, left and right of the jet pump, are then subtracted yielding the time-averaged pressure drop $\Delta p_{2}$ across the jet pump. For the case shown in Fig. 2.6, a total pressure drop of $\Delta p_{2}=28.8 \mathrm{~Pa}$ is generated.

Figure 2.6c shows the axial profile of the acoustic power $\dot{E}_{2}$. The acoustic power is determined by the pressure and velocity fields and can be calculated either by direct time integration of the transient solution or by using the calculated complex wave amplitudes. In the first method all resolved higher order effects will be included while in the latter only the first order acoustics is taken into account. While the integration method will provide the most complete solution, the method based on first order variables is most relevant for thermoacoustic applications as typically only the power transported by the first harmonic will contribute to a device's efficiency and any conversion to higher harmonics are considered losses. ${ }^{15}$ Using the calculated amplitudes of pressure $p_{1}$ and volume flow rate $U_{1}$, the acoustic power is defined as

$$
\dot{E}_{2}(x)=\frac{1}{2} \Re\left[\widetilde{p}_{1}(x) U_{1}(x)\right]
$$

where $\widetilde{p}_{1}(x)$ is the complex conjugate of the pressure amplitude area-averaged over the local cross section. The acoustic power dissipation across the jet pump $\Delta \dot{E}_{2}$ is determined in a similar manner as the time-averaged pressure drop. For the current case this yields $\Delta \dot{E}_{2}=58.8 \mathrm{~mW}$. 

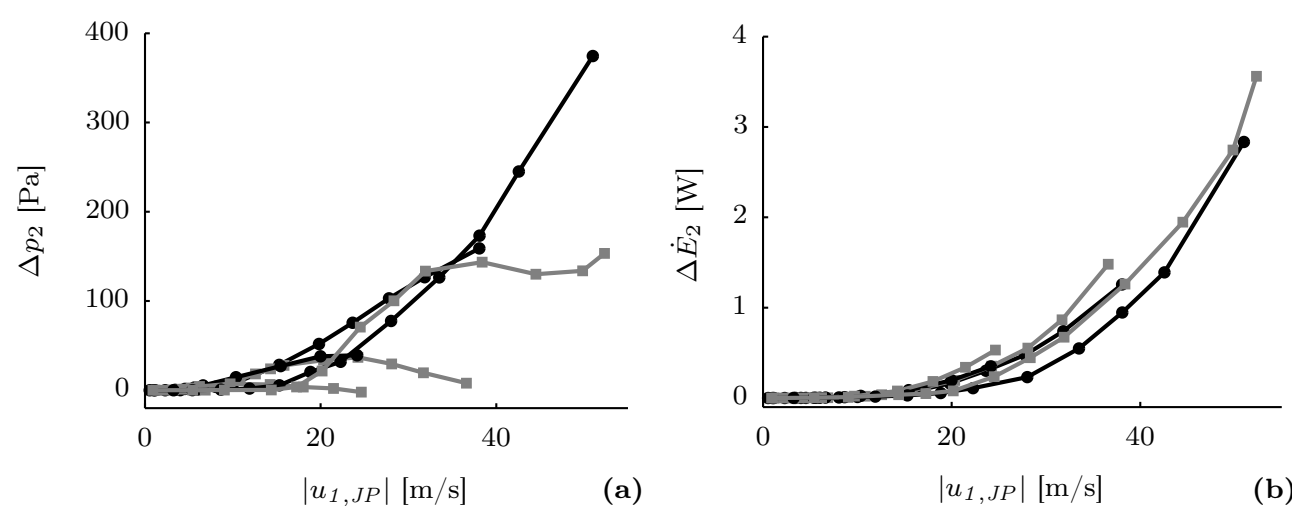

Figure 2.7: (a) Time-averaged pressure drop and (b) acoustic power dissipation for two different jet pump geometries as a function of jet pump waist velocity amplitude, $\alpha=7^{\circ}(\mathbf{\bullet})$ and $\alpha=15^{\circ}(\square)$. Three different frequencies included: $50 \mathrm{~Hz}, 100 \mathrm{~Hz}$ and $200 \mathrm{~Hz}$.

\subsubsection{Jet pump performance}

By varying the far field velocity amplitude, the relation between the jet pump performance and waist velocity is studied. The jet pump waist velocity amplitude ranges from $0.5 \mathrm{~m} / \mathrm{s}$ to $52 \mathrm{~m} / \mathrm{s}$ which corresponds to acoustic displacement amplitudes from $0.4 \mathrm{~mm}$ to $78 \mathrm{~mm}$. From the velocity amplitude, the acoustic Reynolds number is defined as

$$
R e=\frac{\left|u_{1}\right| \delta_{\nu} \rho_{0}}{\mu_{0}} .
$$

Ohmi and Iguchi derived a critical Reynolds number for the transition to turbulence in oscillating pipe flow which can be rewritten to ${ }^{83,84}$

$$
R e_{c}=305\left(\frac{D}{\delta_{\nu}}\right)^{\frac{1}{7}}
$$

The maximum acoustic Reynolds number in the computational domain occurs at the location of the jet pump waist. For the simulated cases presented in the following, this maximum value falls below the critical Reynolds number which verifies the laminar assumption. Table 2.5 shows an overview of the investigated Reynolds numbers and the corresponding critical values.

The time-averaged pressure drop and acoustic power dissipation for the two different jet pump taper angles and three different frequencies are shown in Fig. 2.7. The acoustic power dissipation is similar for both geometries and increases approximately with the cube of $\left|u_{1, J P}\right|$ as was predicted based on the quasi-steady model (Eq. 1.8 in Sec. 1.3.1). However, for the time-averaged pressure drop a large difference can be observed which is a consequence of the change in taper angle or frequency. Where the quasi-steady model predicts a quadratic relation between $\Delta p_{2}$ and $\left|u_{1, J P}\right|$ (Eq. 1.7), this is clearly not the case over the entire range of velocity amplitude for the simulations. For low amplitudes, a nearly quadratic increase is observed for both geometries. 
Table 2.5: Acoustic Reynolds numbers in the jet pump waist and critical Reynolds numbers for the simulated cases.

\begin{tabular}{llll}
\hline$\alpha$ & $f$ & $\max (R e)$ & $R e_{c}$ \\
\hline $7^{\circ}$ & $50 \mathrm{~Hz}$ & 486 & 524 \\
$15^{\circ}$ & $50 \mathrm{~Hz}$ & 496 & 524 \\
$7^{\circ}$ & $100 \mathrm{~Hz}$ & 542 & 551 \\
$15^{\circ}$ & $100 \mathrm{~Hz}$ & 521 & 551 \\
$7^{\circ}$ & $200 \mathrm{~Hz}$ & 513 & 579 \\
$15^{\circ}$ & $200 \mathrm{~Hz}$ & 526 & 579 \\
\hline
\end{tabular}

However, for the $15^{\circ}$ taper angle the pressure drop stagnates and eventually decreases at higher velocity amplitudes. Also, the $7^{\circ}$ taper angle shows a deviation from the theoretical pressure drop profile and stagnates, but this happens at much higher velocity amplitudes compared to the $15^{\circ}$ geometry.

The results at $100 \mathrm{~Hz}$ have been compared against preliminary experimental results that have been achieved using identical jet pump geometries in a traveling wave experimental setup at a driving frequency of $113 \mathrm{~Hz}$. The measured time-averaged pressure drop shows the same behavior as the simulation results for both jet pump geometries. The maximum deviation is less than $20 \%$ in the region where the measured timeaveraged pressure drop is significant (for $\Delta p_{2}>10 \mathrm{~Pa}$ ). The experimental results are further discussed in Chapters 5-6.

The effect of the jet pump geometry and frequency on the time-averaged pressure drop can be explained by scaling the velocity amplitude using the Keulegan-Carpenter numbers based on either the jet pump length or the jet pump waist diameter (Eq. 2.5 and Eq. 2.6, respectively). Moreover, the pressure drop and acoustic power dissipation are scaled according to 64

$$
\begin{gathered}
\Delta p_{2}^{*}=\frac{8 \Delta p_{2}}{\rho_{0}\left|u_{1, J P}\right|^{2}}, \\
\Delta \dot{E}_{2}^{*}=\frac{3 \pi \Delta \dot{E}_{2}}{\rho_{0} \pi R_{s}^{2}\left|u_{1, J P}\right|^{3}},
\end{gathered}
$$

where $\Delta p_{2}^{*}$ would represent the difference in minor loss coefficients between the two flow directions assuming the quasi-steady theory (Eq. 1.7) and $\Delta \dot{E}_{2}^{*}$ would represent the summation of the minor loss coefficients assuming Eq. 1.8 to be valid.

The onset of the jet pump working is found to be determined by the KeuleganCarpenter number based on the jet pump waist diameter, $K C_{D}$, and is shown in Fig. 2.8 for the two investigated taper angles and three different driving frequencies. No time-averaged pressure drop is measured for low values $\left(K C_{D}<0.5\right)$. This corresponds to the flow regime described in Fig. 2.4a where there are no minor loss inducing flow phenomena observed. In the low amplitude regime, the effect of the jet pump taper angle is negligible and both geometries follow the same line. However, at higher amplitudes the curves become deviant and the effect of jet pump taper angle 


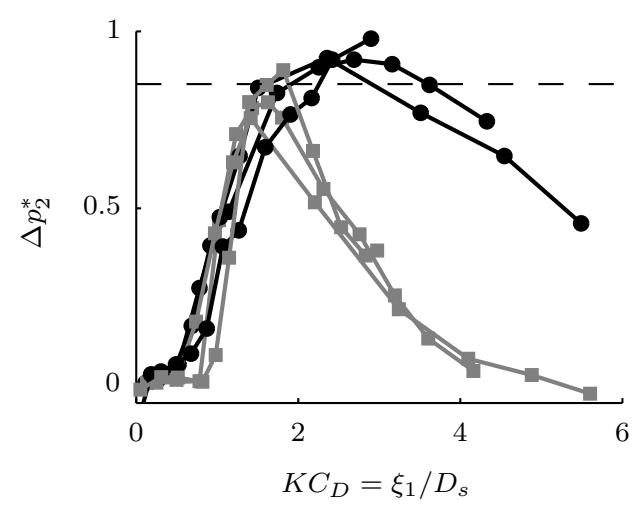

Figure 2.8: Dimensionless pressure drop $\Delta p_{2}^{*}$ as a function of $K C_{D}$ for two different jet pump geometries: $\alpha=7^{\circ}(\mathbf{O})$ and $\alpha=15^{\circ}(\square)$ at $50 \mathrm{~Hz}, 100 \mathrm{~Hz}$ and $200 \mathrm{~Hz}$. Dashed line indicates quasi-steady approximation.
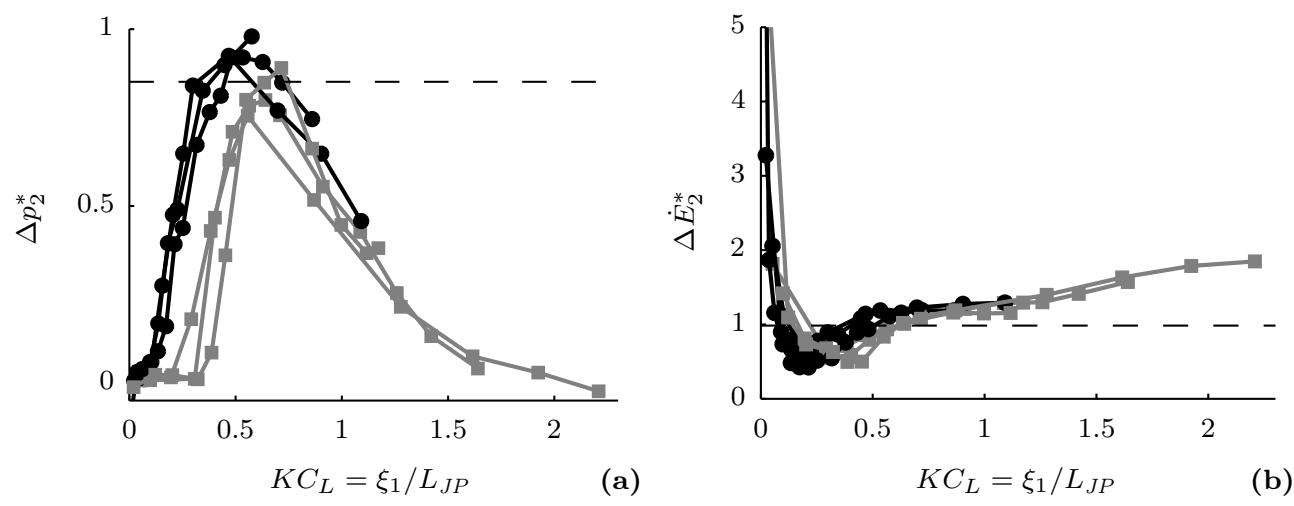

Figure 2.9: (a) Dimensionless pressure drop and (b) dimensionless acoustic power dissipation as a function of $K C_{L}$ for two different jet pump geometries: $\alpha=7^{\circ}(\bullet)$ and $\alpha=15^{\circ}(\square)$ at $50 \mathrm{~Hz}$, $100 \mathrm{~Hz}$ and $200 \mathrm{~Hz}$. Dashed lines indicate quasi-steady approximation.

becomes apparent which is well accounted for by using $K C_{L}$ rather than $K C_{D}$. Note that the effect of frequency is well accounted for by using the acoustic displacement amplitude as a scaling parameter rather than the velocity amplitude.

Figure 2.9 shows the dimensionless quantities $\Delta p_{2}^{*}$ and $\Delta \dot{E}_{2}^{*}$ as a function of $K C_{L}$. Both jet pump taper angles show the same trend indicating that the jet pump performance is directly related to the jet pump length. A maximum in the dimensionless pressure drop is visible around $K C_{L} \approx 0.5$ for both geometries. As soon as the displacement amplitude becomes large with respect to the jet pump length, the total minor loss coefficient begins to decrease again. This corresponds well to the observed asymmetry in the flow fields as discussed in Section 2.3.1. At $K C_{L} \approx 0.5$ the flow field is highly asymmetric between both sides of the jet pump (Fig.2.4c) while for $K C_{L}>1$ a large part of the asymmetry is lost and flow separation occurs. The time-averaged separated flow reduces the "effective" diameter of the big opening (Fig. 2.4d). This 
will consequently lead to more symmetric minor losses and hence to a decrease in the time-averaged pressure drop while the acoustic power dissipation will still increase. In this regime, the quasi-steady approximation is not valid anymore and an adjustment is required.

Comparing the obtained maximum value of $\Delta p_{2}^{*}$ with the quasi-steady approximation (dashed line) in Fig. 2.9a, a close match is observed for both taper angles. This suggests the quasi-steady approximation can be used to predict the time-averaged pressure drop but only for the optimal situation where $K C_{L} \approx 0.5$. The acoustic power dissipation is predicted well for all cases by the quasi-steady model as shown in Fig. 2.9b. The assumed cubic relation between $\Delta \dot{E}_{2}$ and $\left|u_{1, J P}\right|$ is confirmed by the fairly constant value of $\Delta \dot{E}_{2}^{*}$, especially for $0.5<K C_{L}<1.5$. In this region, the jet pump acts as a pure acoustic resistance and its behavior is not affected by a change in flow regime.

\subsection{Conclusions}

A computational fluid dynamics model is successfully used to simulate the oscillatory flow through two different jet pump geometries under traveling wave conditions. The relation between the obtained time-averaged pressure drop and the acoustic displacement amplitude is investigated together with the acoustic power dissipation.

Four different flow regimes are distinguished based on the Keulegan-Carpenter numbers and the observed flow phenomena are related to the jet pump performance. For $K C_{D}<0.5$ no vortex shedding is observed, resulting in a negligible pressure drop. At $K C_{L} \approx 0.5$, the highly asymmetric flow field leads to a substantial pressure drop and a steady jet to the right side of the jet pump is observed. The measured jet pump performance in this regime corresponds well with the quasi-steady approximation.

When $K C_{L}>1$, vortices are shed through the jet pump to the left resulting in an additional jet to the left side of the jet pump and flow separation in the jet pump is observed. Reducing the asymmetry of the flow field consequently leads to a decay in the time-averaged pressure drop but increases the acoustic power dissipation. In this flow regime, an adjustment to the quasi-steady approximation is required to reliably predict the jet pump performance.

Several additional geometric parameters have not been considered in this chapter and additional research is required in order to distinguish more precisely between the different flow phenomena. This should provide insight into whether the decay in $\Delta p_{2}^{*}$ for high values of $K C_{L}$ is solely caused by the jet pump length or by the jet pump taper angle as was postulated in previous work. ${ }^{61}$ Moreover, the influence of the radius of curvature on the jet pump performance is not investigated here. The radius of curvature is expected to have an effect on the flow separation inside the jet pump and consequently on the overall performance.

A first step towards a better understanding of the physics behind jet pumps is made 
but more attention is yet required to reliably predict a jet pump's performance. A thorough numerical parameter study on the various (two-dimensional) geometric parameters together with experimental research on three-dimensional geometry variations are the next steps to be undertaken to provide insight into the jet pump scaling problem. 


\section{Geometric parameter study on the laminar jet pump performance}

The oscillatory flow through jet pumps is characterized by a numerical parameter study. The two-dimensional axisymmetric computational fluid dynamics model introduced in Chapter 2 is used to calculate the performance of a large number of conical jet pump geometries in terms of time-averaged pressure drop and acoustic power dissipation. The investigated geometrical parameters include the jet pump length, taper angle, waist diameter and waist curvature. In correspondence with the findings of Chapter 2, four flow regimes are observed which characterize the jet pump performance and dimensionless parameters are introduced to scale the performance of the various jet pump geometries. Based on the scaling parameters, an optimum operation region is defined and design guidelines are proposed which can be directly used for future jet pump design.

\subsection{Introduction}

Although some jet pump measurements are available in literature (see Section 1.3.2), a systematic parameter study that directly relates variations in wave amplitude and geometry to a jet pump's performance has not yet been addressed. A first step towards the investigation of a jet pump's performance in oscillatory flows has been made in Chapter 2 by scaling the jet pump geometry using two different Keulegan-Carpenter numbers and correlating this to the jet pump performance. ${ }^{73}$

Albeit the described flow regimes were distinguished using the proposed scaling, the data set was too limited to determine whether the observed flow separation (Fig. 2.4d

Adapted from J. P. Oosterhuis, S. Bühler, D. Wilcox, and T. H. van der Meer. "Jet pumps for thermoacoustic applications: design guidelines based on a numerical parameter study." J. Acoust. Soc. Am., 138(4):1991-2002, 2015. doi:10.1121/1.4929937 
on page 20) was geometrically initiated by an increase in the taper angle or by a decrease in the jet pump length. In this chapter, a parameter study is performed using the same computational fluid dynamics (CFD) model that was introduced in Chapter 2 to further identify the relation between the four different flow regimes and the jet pump geometry. The influence of various geometric parameters including the jet pump taper angle, length, curvature and waist diameter on the jet pump performance is investigated. Based on the presented results, the existing scaling parameters are further extended. Furthermore, a comparison with the quasi-steady model is provided to determine under what conditions the approximation is applicable. Based on this parameter study, design guidelines for future jet pump design are proposed.

After a brief description of the used CFD model in Section 3.2, the various investigated jet pump geometries are introduced in Section 3.2.2. The resulting flow regimes will be distinguished in Section 3.3.1 and subsequently linked to the jet pump performance in Section 3.3.2. Finally, the influence of the jet pump curvature is investigated in Section 3.3.3 before drawing final conclusions on the design guidelines in Section 3.4.

\subsection{Modeling}

An axisymmetric CFD model is used which has been introduced in Chapter 2 on page 14. The jet pump samples are placed in an outer tube which has a radius of $R_{0}=30 \mathrm{~mm}$. No-slip adiabatic wall boundary conditions are imposed at the jet pump walls while the walls of the outer tube are modeled using a slip adiabatic boundary condition. The acoustic wave is generated using an oscillatory velocity boundary condition at the left boundary and the acoustic waves can leave the domain freely without reflections using a dedicated time-domain impedance boundary condition on the right side of the domain (see Sec. 2.2.3 and Appendix. A).

\subsubsection{Simulation of flow separation}

The choice for an axisymmetric model to simulate flow separation might need some additional explanation as in planar diffusers the flow separation can be asymmetric. ${ }^{86,87}$ A clear distinction should be made between flow separation in planar and conical diffusers. The steady flow separation in conical diffusers has been investigated previously and no visible asymmetry of the flow was reported in laminar conditions. ${ }^{63}$ Furthermore, the oscillatory nature of the flow prevents the flow from developing asymmetric instabilities, even in planar diffuser geometries. ${ }^{62,88}$ These observations motivate the applicability of an axisymmetric model for the current situation of pure oscillatory flow through a conical geometry.

In addition to the mesh validation performed in previous work, ${ }^{73}$ the accuracy of the employed computational mesh has been investigated specifically for the prediction of flow separation. The jet pump geometry with a taper angle of $\alpha=15^{\circ}$ (No. 4 in Table 3.2) has been used at a frequency of $100 \mathrm{~Hz}$ and at a wave amplitude where 


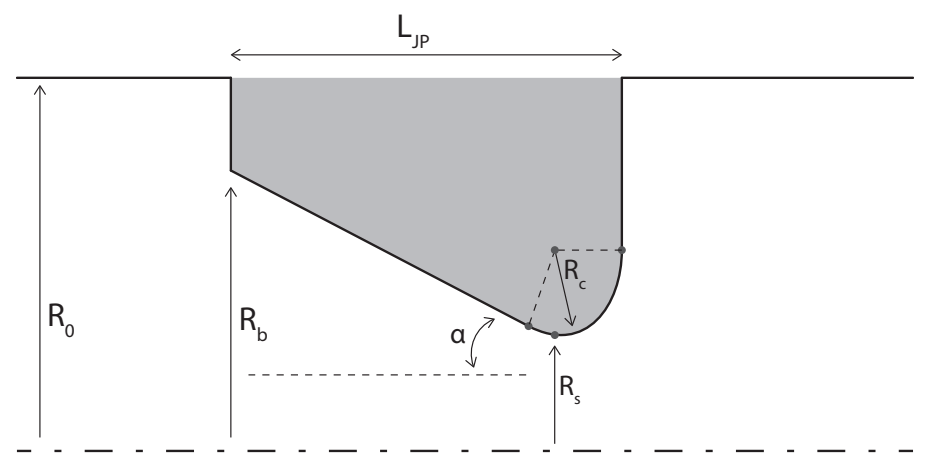

Table 3.1: Dimensions of the reference jet pump geometry.

Figure 3.1: Jet pump with parameters that define the geometry (not to scale). Bottom dashed line indicates center line, top solid line indicates outer tube wall.

\begin{tabular}{ll}
\hline$R_{0}$ & $30 \mathrm{~mm}$ \\
$R_{b}$ & $15 \mathrm{~mm}$ \\
$R_{s}$ & $7 \mathrm{~mm}$ \\
$R_{c}$ & $5 \mathrm{~mm}$ \\
$L_{J P}$ & $70.5 \mathrm{~mm}$ \\
$\alpha$ & $7^{\circ}$ \\
\hline
\end{tabular}

flow separation is expected $\left(K C_{\alpha}=0.85\right.$, Eq. 3.2). The mesh size in the jet pump region is in two steps refined from $1 \mathrm{~mm}$ to $0.5 \mathrm{~mm}$ and $0.25 \mathrm{~mm}$. At the same time, the mesh size in the jet pump waist region is refined from $0.5 \mathrm{~mm}$ to $0.2 \mathrm{~mm}$ and $0.1 \mathrm{~mm}$. Additionally, the effect of a mesh refinement in the viscous boundary layer is investigated by increasing the number of elements from 10 to 20 . The difference in the dimensionless pressure drop (Eq. 3.5) and dimensionless acoustic power dissipation (Eq. 3.6) between the investigated meshes is less than 0.04 and 0.05 , respectively. Furthermore, both the time and location where the flow first separates do not vary more than $\Delta t / T=8 \cdot 10^{-3}$ and $\Delta x=0.4 \mathrm{~mm}$ between the different mesh resolutions.

\subsubsection{Jet pump geometries}

The described computational model is used to investigate a large variety of conical jet pump geometries and wave amplitudes. By varying either the jet pump length, the size of one of the two openings or the local curvature at the jet pump waist, 19 different geometries are considered. The different investigated geometries are all variations based on one reference geometry (denoted by "ref" in all tables). The reference geometry is a jet pump with a $7^{\circ}$ taper angle and identical to the geometry presented in Chapter 2. All its dimensions are denoted in Table 3.1 and correspond to the parameters indicated in Fig. 3.1.

Varying the jet pump taper angle while keeping the waist diameter constant can be achieved by either changing the jet pump length $L_{J P}$ or changing the radius of the big opening $R_{b}$. Because the jet pump length is not taken into account in the quasi-steady approximation (Section 1.3.1), the predicted jet pump performance is independent of the jet pump length. In a similar way, the radius of the big opening is only a weak parameter in the quasi-steady approximation as long as $R_{b} \gg R_{s}$. Both approaches are considered here to see what actually influences the jet pump performance: the distance between the two openings $\left(L_{J P}\right)$ or the taper angle of the jet pump inner 
Table 3.2: Dimensions of jet pump geometries with varied taper angles $\alpha$ by either changing the jet pump length or the big radius. Constant radius of the jet pump waist $R_{s}=7.0 \mathrm{~mm}$ and constant curvature of the small opening $R_{c}=5.0 \mathrm{~mm}$.

\begin{tabular}{lclll}
\hline No. & symbol & $\alpha$ & $L_{J P}$ & $R_{b}$ \\
\hline 1 & $\bullet$ & $3^{\circ}$ & $157.8 \mathrm{~mm}$ & $15.0 \mathrm{~mm}$ \\
2 & $\mathbf{\square}$ & $5^{\circ}$ & $96.7 \mathrm{~mm}$ & $15.0 \mathrm{~mm}$ \\
ref & $\times$ & $7^{\circ}$ & $70.5 \mathrm{~mm}$ & $15.0 \mathrm{~mm}$ \\
3 & $\boldsymbol{\nabla}$ & $10^{\circ}$ & $50.8 \mathrm{~mm}$ & $15.0 \mathrm{~mm}$ \\
4 & $\bullet$ & $15^{\circ}$ & $35.5 \mathrm{~mm}$ & $15.0 \mathrm{~mm}$ \\
5 & $\bullet$ & $20^{\circ}$ & $27.9 \mathrm{~mm}$ & $15.0 \mathrm{~mm}$ \\
\hline 6 & $\bullet$ & $3^{\circ}$ & $70.5 \mathrm{~mm}$ & $10.4 \mathrm{~mm}$ \\
7 & $\mathbf{\square}$ & $5^{\circ}$ & $70.5 \mathrm{~mm}$ & $12.7 \mathrm{~mm}$ \\
8 & $\boldsymbol{\nabla}$ & $10^{\circ}$ & $70.5 \mathrm{~mm}$ & $18.5 \mathrm{~mm}$ \\
9 & $\bullet$ & $15^{\circ}$ & $70.5 \mathrm{~mm}$ & $24.4 \mathrm{~mm}$ \\
\hline
\end{tabular}

Table 3.3: Dimensions of jet pump geometries with varied waist radius $R_{s}$. Constant taper angle $\alpha=7^{\circ}$ and jet pump length $L_{J P}=70.5 \mathrm{~mm}$.

\begin{tabular}{llll}
\hline No. & $R_{s}$ & $R_{b}$ & $R_{c}$ \\
\hline 10 & $3.0 \mathrm{~mm}$ & $11.4 \mathrm{~mm}$ & $2.1 \mathrm{~mm}$ \\
11 & $5.0 \mathrm{~mm}$ & $13.2 \mathrm{~mm}$ & $3.6 \mathrm{~mm}$ \\
ref & $7.0 \mathrm{~mm}$ & $15.0 \mathrm{~mm}$ & $5.0 \mathrm{~mm}$ \\
12 & $9.0 \mathrm{~mm}$ & $16.8 \mathrm{~mm}$ & $6.4 \mathrm{~mm}$ \\
13 & $11.0 \mathrm{~mm}$ & $18.6 \mathrm{~mm}$ & $7.9 \mathrm{~mm}$ \\
\hline
\end{tabular}

surface $(\alpha)$. Table 3.2 shows the dimensions of the investigated geometries where the taper angle is changed with respect to the reference case by either varying the jet pump length (cases 1-5) or by varying the big radius (cases $6-9$ ). The jet pump waist radius and curvature are kept constant at $R_{s}=7 \mathrm{~mm}$ and $R_{c}=5 \mathrm{~mm}$, respectively. This results in a dimensionless curvature of $\chi=R_{c} / D_{s}=0.36$ which is considered a "smooth" contraction. ${ }^{58}$ Correspondingly, the contraction minor loss coefficient at the jet pump waist $\left(K_{c o n, s}\right.$ in Eq. 1.7) is expected to be negligible which will enhance the overall time-averaged pressure drop.

In a different set of simulations, both the taper angle and the jet pump length are fixed while the jet pump waist diameter is changed (cases 10-13). Furthermore, the jet pump curvature is adjusted to maintain the same dimensionless curvature. The dimensions of this set of geometries are shown in Table 3.3.

In the following, the observed flow regimes and jet pump performance will be discussed for the cases where either the jet pump length or the size of one of the two openings is varied. In Section 3.3.3, the effect of the jet pump waist curvature will be investigated. 


\subsection{Results and discussion}

For each geometry, various wave amplitudes and consequently, various displacement amplitudes are simulated. The wave amplitude is varied by specifying different values of the velocity amplitude at the left boundary condition. It is important to ensure that the simulated wave amplitudes fall within the laminar regime as URANS turbulence models, especially in combination with wall functions, are known to lose their validity for relaminarizing and separating flows which is an important part of the current data set. ${ }^{86,89,90}$ Other approaches to model turbulence such as large eddy simulation (LES) or direct numerical simulation (DNS) are currently not feasible for a parameter study of the size presented here. In order to determine what wave amplitudes fall within the laminar regime, the critical Reynolds number derived by Ohmi and Iguchi for oscillatory pipe flow is used, ${ }^{83,84}$

$$
R e_{c}=305\left(\frac{D}{\delta_{\nu}}\right)^{\frac{1}{7}}
$$

where the Reynolds number is defined based on the viscous penetration depth: $R e=$ $\left|u_{1}\right| \delta_{\nu} \rho_{0} / \mu_{0}$. Normally, the velocity amplitude is directly proportional to the frequency for a given displacement amplitude, but the velocity amplitude where a transition to turbulence occurs $\left(R e=R e_{c}\right)$ scales with $f^{4 / 7}$. In Chapter 2 , it was concluded that the jet pump performance scales with the acoustic displacement amplitude instead of the jet pump velocity amplitude. Consequently, by lowering the driving frequency all displacement amplitudes of interest are investigated. The majority of the presented simulations are carried out with $f=100 \mathrm{~Hz}$ while some additional simulations are using frequencies of $10 \mathrm{~Hz}, 20 \mathrm{~Hz}, 30 \mathrm{~Hz}, 50 \mathrm{~Hz}$ and $200 \mathrm{~Hz}$. All data presented in this chapter represents a total of 197 simulations having a total single core computational time of 391 days on an Intel Core i7 CPU.

From the simulated jet pump performance, a scaling parameter is introduced which best aligns the jet pump performance for the various investigated geometries and correctly incorporates the effect of the jet pump taper angle,

$$
K C_{\alpha}=\frac{\xi_{1}}{D_{s}} \cdot \alpha
$$

This scaling parameter is essentially the Keulegan-Carpenter number (Eq. 2.6) multiplied by the taper angle (in radians). Note that this is different from the scaling parameter $K C_{L}$ that was postulated in Chapter 2. The results in the following section will show that it is the taper angle initiating flow separation rather than the length of the jet pump by comparing results from jet pump geometries No. 1-4 to geometries No. 6-9.

\subsubsection{Flow regimes}

Because the jet pump performance is found to be a strong function of the flow regime, the observed flow regimes will be discussed first. By studying the transient vorticity 

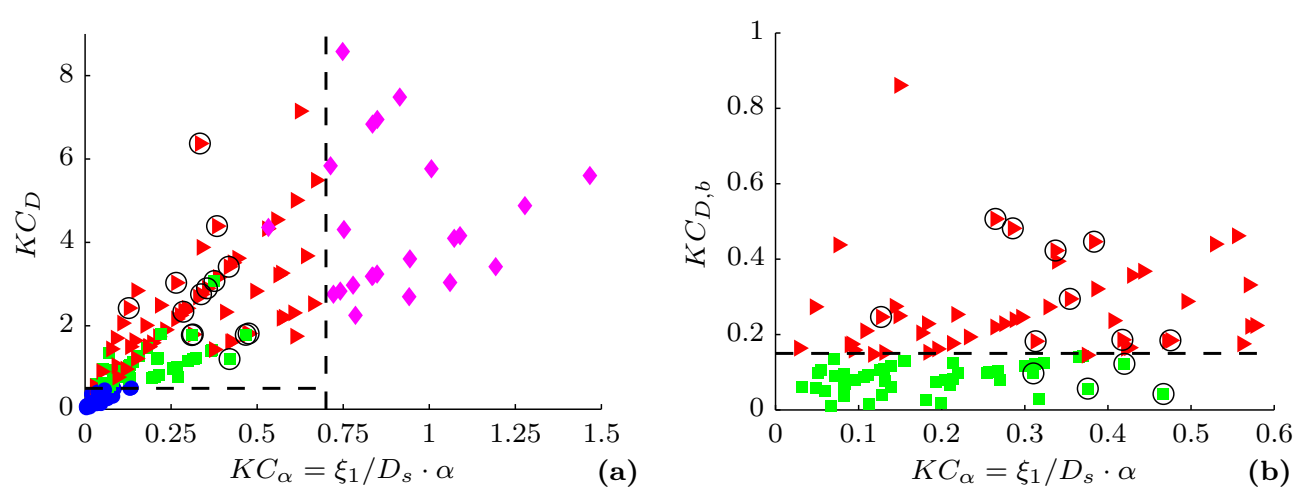

Figure 3.2: Observed flow regimes using jet pump geometries with varied taper angle and waist diameter. (a) All four flow regimes with $K C_{D}$ on the vertical axis. (b) Flow regimes with one-sided and two-sided vortex propagation shown against local Keulegan-Carpenter number at the big jet pump opening, $K C_{D, b}$. The different flow regimes are distinguished using different symbols: - for oscillatory vortices, $\square$ for right-side vortex propagation only, $\boldsymbol{\nabla}$ for two-sided vortex propagation and $\diamond$ for flow separation. The dashed lines represent the determined bounds of the flow regimes and the open circles indicate the points where the maximum $\Delta p_{2}^{*}$ was found for a specific jet pump geometry.

field and streaming velocity field of all simulations, a differentiation is made between the four flow regimes that have been illustrated in Fig. 2.4 on page 20. The flow regimes that have been observed using various wave amplitudes and jet pump geometries are all presented in the $\left(K C_{\alpha}, K C_{D}\right)$ space in Fig. 3.2a. As such, the horizontal axis includes the taper angle while the vertical axis represents the Keulegan-Carpenter number without the contribution of the taper angle. The different symbols represent the different flow regimes: circles for oscillatory vortex pairs, squares for right-sided vortex propagation, triangles for two-sided vortex propagation and diamonds for flow separation and alternating vortex shedding. The open circles indicate the position where the maximum dimensionless pressure drop was achieved with a specific jet pump geometry.

Two flow regimes are easily distinguished in Fig. 3.2a. For small values of the Keulegan-Carpenter number, $K C_{D}<0.5$, no vortex shedding is observed. This is indicated by the horizontal dashed line in Fig. 3.2a. In this regime, no asymmetry in minor losses occurs and consequently the time-averaged pressure drop is zero. The transition from this flow regime to the situation where vortex shedding is first observed is independent of the taper angle and occurs when $K C_{D}>0.5$. Above this value the flow field becomes asymmetric resulting in a positive time-averaged pressure drop. This is in line with the work of Holman et al. on synthetic jets where a formation criterion for vortex shedding is derived of the form $K C_{D}=R e_{D} / S^{2}>C$ where $C$ is a constant depending on the geometry of the orifice. ${ }^{68}$

In Fig. 3.2a the flow separation regime (diamonds) can be distinguished from the other three flow regimes and occurs when $K C_{\alpha}>0.7$ (dashed vertical line). Because this transition is clearly determined by the scaling parameter as introduced in Eq. 3.2, the taper angle plays an important role in the initiation of the flow separation regime. 
This flow regime is dominated by vortices being shed from the jet pump waist in alternating directions. This results in the flow field again becoming more and more symmetric. Despite the interesting flow phenomena occurring in this flow regime, ${ }^{73}$ it is of little practical interest when designing jet pumps as the achieved jet pump effectiveness is low. Consequently, the area of interest for jet pump applications is bounded to $K C_{D}>0.5$ and $K C_{\alpha}<0.7$.

Inside this area, two flow regimes occur which cannot solely be separated based on $K C_{D}$ or $K C_{\alpha}$. Whether vortex shedding from the big jet pump opening occurs, is found to be dependent on the local displacement amplitude at the position of the big opening $\xi_{1, b}$. A Keulegan-Carpenter number at the big jet pump opening is estimated using

$$
K C_{D, b}=\frac{\xi_{1} \frac{A_{s}}{A_{b}}}{D_{b}}=\xi_{1} \frac{R_{s}^{2}}{2 R_{b}^{3}} .
$$

Note that $\xi_{1}$ is still the local displacement amplitude at the jet pump waist. Figure $3.2 \mathrm{~b}$ shows the flow regimes where either one-sided or two-sided vortex propagation occurs with $K C_{D, b}$ instead of $K C_{D}$ on the vertical axis. Only the range $0<K C_{\alpha}<0.7$ is shown, omitting the flow separation regime. The two-sided vortex propagation is first observed when $K C_{D, b}>0.15$. This is at a different value than where vortex propagation at the jet pump waist was first recognized $\left(K C_{D}>0.5\right)$, which is an effect of the local dimensionless curvature $\chi$. The observed transition at $K C_{D, b}=0.15$ very nicely corresponds to the value found experimentally for a sharpedged axisymmetric orifice. ${ }^{68}$ The effect of the jet pump curvature will be further investigated in Section 3.3.3.

\subsubsection{Jet pump performance}

Ideally, a jet pump should impose the desired time-averaged pressure drop while having minimum acoustic power dissipation. Thus, the jet pump performance can be determined using two quantities: the time-averaged pressure drop $\Delta p_{2}$ and the acoustic power dissipation $\Delta \dot{E}_{2}$, both a function of the jet pump waist velocity amplitude $\left|u_{1, J P}\right|$. The time-averaged pressure drop over the jet pump is calculated from the simulation results by a subtraction of the mean pressure fields on either side of the jet pump. In this way the influence of the vortex propagation distance on the time-averaged pressure drop is eliminated. ${ }^{73}$

The acoustic power is calculated using first order quantities as ${ }^{15}$

$$
\dot{E}_{2}(x)=\frac{1}{2} \Re\left[\tilde{p}_{1}(x) U_{1}(x)\right]
$$

with $\tilde{p}_{1}(x)$ the complex conjugate of the pressure amplitude area-averaged over the local cross section and $U_{1}(x)$ the complex volume flow rate. The dissipation of acoustic power across the jet pump, $\Delta \dot{E}_{2}$, is determined in a similar manner as the timeaveraged pressure drop. 
To better understand the relation between these two quantities, a scaling is applied according to the work of Smith and Swift, ${ }^{64}$

$$
\begin{gathered}
\Delta p_{2}^{*}=\frac{8 \Delta p_{2}}{\rho_{0}\left|u_{1, J P}\right|^{2}}, \\
\Delta \dot{E}_{2}^{*}=\frac{3 \pi \Delta \dot{E}_{2}}{\rho_{0} \pi R_{s}^{2}\left|u_{1, J P}\right|^{3}} .
\end{gathered}
$$

Note that subject to the quasi-steady approximation, $\Delta p_{2}^{*}$ would represent the difference in minor loss coefficients between the two flow directions while $\Delta \dot{E}_{2}^{*}$ would represent the summation of the minor loss coefficients (i.e. the last terms in Eq. 1.7 and 1.8 on page 9 , respectively). Using these two dimensionless quantities, a jet pump effectiveness can be defined as ${ }^{64}$

$$
\eta=\frac{\Delta p_{2}^{*}}{\Delta \dot{E}_{2}^{*}} .
$$

Optimal jet pump performance is achieved when $\eta$ is maximized. These three performance characteristics are calculated for each simulation case. In the following, they will be shown as a function of the scaling parameters introduced previously to study the effect of geometry changes on the jet pump performance.

\section{Time-averaged pressure drop}

Figure $3.3 \mathrm{a}$ shows the dimensionless pressure drop $\Delta p_{2}^{*}$ as a function $K C_{\alpha}$. The symbols represent different taper angles, obtained by either changing the jet pump length or the big radius, each depicted in Table 3.2. The results with a varied waist diameter are all plotted using crosses $(\times)$, representing their taper angle of $\alpha=7^{\circ}$. The maximum dimensionless pressure drop for all cases is achieved between $0.2<$ $K C_{\alpha}<0.5$ which fits well within the determined range of the flow regimes where vortex shedding, but no flow separation, occurs. This emphasizes the direct relation between the flow field asymmetry and the time-averaged pressure drop.

A decay in $\Delta p_{2}^{*}$ is observed for higher values of $K C_{\alpha}$. The range where flow separation was observed coincides with this decay in dimensionless pressure drop. The decay follows the same trend for all jet pump geometries and is properly scaled using the adjusted Keulegan-Carpenter number, which shows the dependency of the flow separation occurrence on the taper angle. Moreover, a corresponding decay in $\Delta p_{2}^{*}$ as a function of $K C_{\alpha}$ is observed in preliminary experimental results that have been obtained at $R e<R e_{c}$ using the $15^{\circ}$ jet pump geometry in an oscillatory flow facility. ${ }^{75,91}$ By performing hot-wire anemometry near the big jet pump opening the existence of an outward directed vortex street was revealed when $K C_{\alpha}>0.7$, confirming the observations from the current simulations. A detailed discussion of the experimental results is deferred to Chapter 5 .

In the published data of Petculescu and Wilen however, the decay in dimensionless pressure drop is less severe. ${ }^{60}$ Their values of $\Delta p_{2}^{*}$ typically stabilize between 0.20 

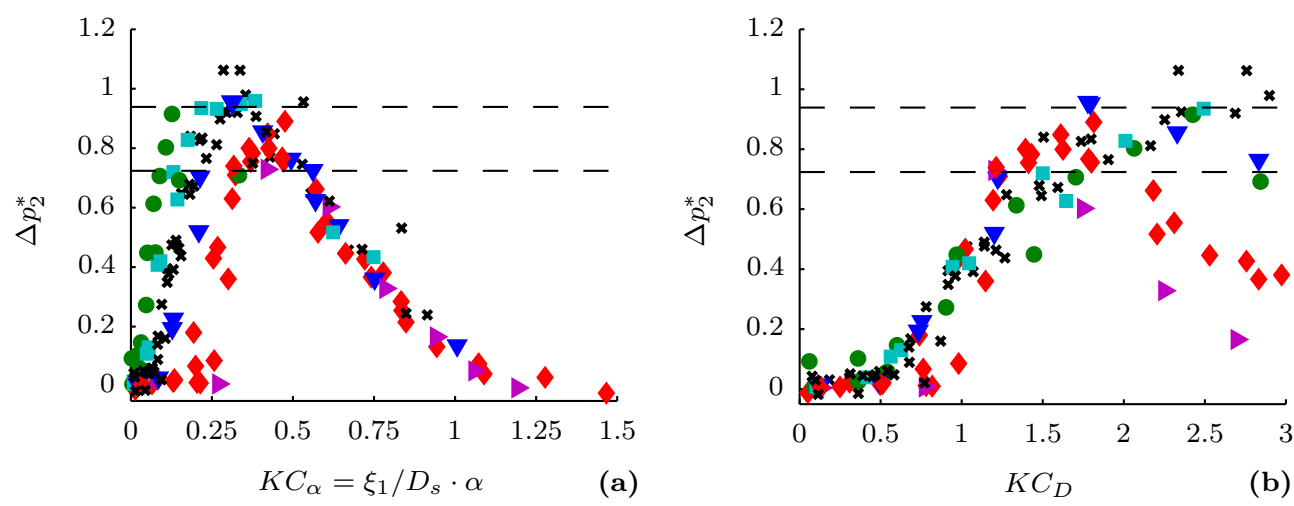

Figure 3.3: Dimensionless pressure drop using jet pump geometries with varied taper angle (Table 3.2) or waist diameter (Table 3.3) as a function of (a) $K C_{\alpha}$ and (b) $K C_{D}$ for $K C_{D}<3$. The symbols represent different taper angles as listed in Table 3.2. The thin dashed lines indicate the upper and lower values of $\Delta p_{2}^{*}$ from the quasi-steady approximation (Eq. 1.7) for the various geometries.

and 0.50 at high values of $K C_{\alpha}$, depending on the taper angle used, and do not decrease to zero. Two possible explanations will be discussed: their small waist diameter compared to the viscous penetration depth and a transition to turbulence. In the geometries of Petculescu and Wilen, $D_{s} / \delta_{\nu} \approx 8.3$ while for the current geometries $D_{s} / \delta_{\nu}$ ranges from 27 to 98 . When the waist diameter is on the order of the viscous penetration depth, the occurrence of flow separation, and consequently the dimensionless pressure drop at $\xi_{1} / D_{s} \cdot \alpha>0.7$, is most likely affected due to viscous effects dominating the flow and preventing the flow from separating. Alternatively, turbulence can affect the decay in $\Delta p_{2}^{*}$ when the turbulent boundary layer prevents the flow from separating. ${ }^{92}$ An investigation of the effect of turbulence on the oscillatory flow in jet pumps is presented in Chapter 5 .

The adjusted Keulegan-Carpenter number, $K C_{\alpha}$, provides consistent scaling of the dimensionless pressure drop in the decaying part of the curve shown in Fig. 3.3a. However, a large spread from the reference geometry is observed for low values of the adjusted Keulegan-Carpenter number $\left(K C_{\alpha}<0.2\right)$. Figure 3.3b shows the dimensionless pressure drop as a function of $K C_{D}$ which better scales the increase of $\Delta p_{2}^{*}$ towards its maximum. This shows that the initial increase of $\Delta p_{2}^{*}$ towards its maximum is not dependent on the taper angle at all. A clear similarity in this graph is the range $K C_{D}<0.5$ where the pressure drop is negligible, corresponding to the flow regime with oscillatory vortices shown in Fig. 3.2a. At larger values of $K C_{D}$, the effect of the taper angle becomes apparent and the results of the different geometries deviate from each other.

\section{Acoustic power dissipation}

By studying the dimensionless acoustic power dissipation, it is observed that the jet pump taper angle has less effect on this performance indicator compared to the 


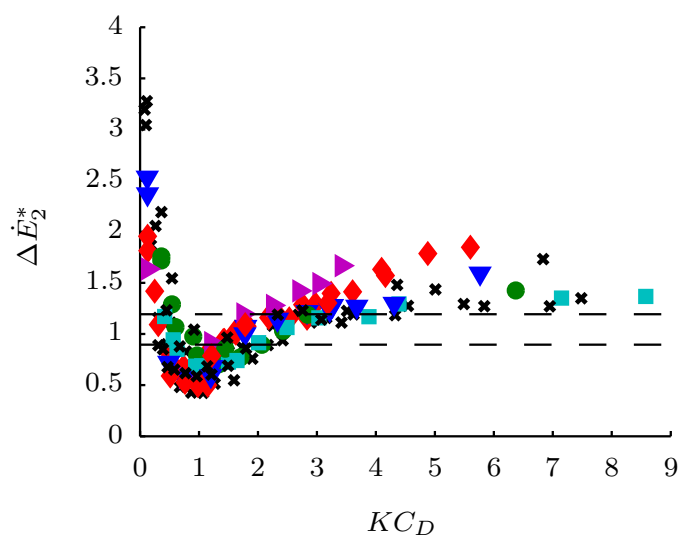

Figure 3.4: Dimensionless acoustic power dissipation using jet pump geometries with varied taper angle (Table 3.2) or waist diameter (Table 3.3). Symbols in accordance with Fig. 3.3. The thin dashed lines indicate upper and lower values of the predicted acoustic power dissipation according to the quasi-steady approximation (Eq. 1.8).

pressure drop. Instead, the dimensionless acoustic power dissipation $\left(\Delta \dot{E}_{2}^{*}\right)$ shows good similarity between the different jet pump geometries when scaled using $K C_{D}$. Figure 3.4 shows the dimensionless acoustic power dissipation as a function of the Keulegan-Carpenter number based on the jet pump waist diameter, $K C_{D}$. The symbols correspond to those in Fig. 3.3. A common minimum occurs around $K C_{D} \approx 1$ while at higher amplitudes $\left(K C_{D}>3\right) \Delta \dot{E}_{2}^{*}$ only slightly increases. Although the taper angle has no effect on the location of the minimum dimensionless acoustic power dissipation, the slight increase of $\Delta \dot{E}_{2}^{*}$ at higher amplitudes is easily observed for higher taper angles $\left(15^{\circ}, \downarrow\right.$ and $\left.20^{\circ}, \boldsymbol{}\right)$.

\section{Comparison to quasi-steady model}

The obtained simulation results can all be compared to the quasi-steady model proposed by Backhaus and Swift. ${ }^{7}$ In this model, the values of $\Delta p_{2}^{*}$ and $\Delta \dot{E}_{2}^{*}$ are assumed to be amplitude independent and the only significant variation in the quasi-steady model occurs by changing the waist diameter. In Fig. 3.3-3.4 the upper and lower bounds of these quasi-steady approximated values are indicated by the thin dashed lines. It becomes clear that the quasi-steady approximation is a good approach to estimate the time-averaged pressure drop of a jet pump when operating in the optimum ranges of $K C_{\alpha}$ and $K C_{D}$. Outside of these ranges however, the actual performance will deviate significantly from the predicted values. The simulated acoustic power dissipation is especially in good agreement for the lower taper angles and $K C_{D}>2$. For high taper angles $\left(15^{\circ}\right.$, and $\left.20^{\circ}, \boldsymbol{}\right)$ however, a deviation from the quasi-steady model is observed at $K C_{D}>2$. On average the quasi-steady values show considerable correspondence to the measured dimensionless acoustic power dissipation. 


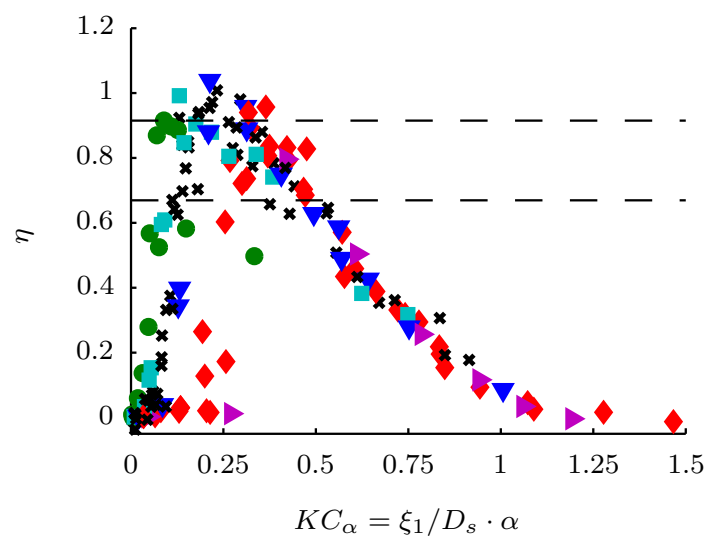

Figure 3.5: Jet pump effectiveness for geometries with varied taper angle (Table 3.2) or waist diameter (Table 3.3). Symbols in accordance with Fig. 3.3.

\section{Jet pump effectiveness}

The two jet pump performance characteristics, dimensionless pressure drop and acoustic power dissipation, can be combined into one effectiveness parameter $\eta$ as defined in Eq. 3.7. Figure 3.5 shows the jet pump effectiveness for all geometries with varied taper angle or waist diameter. The highest jet pump effectiveness is again obtained between $0.2<K C_{\alpha}<0.5$. Note that this does not strictly coincide with the minimum in the acoustic power dissipation (at $K C_{D} \approx 1$ ). However, as long as $K C_{D}>0.5$, the variations in dimensionless pressure drop have a larger influence on the jet pump effectiveness than the relative small changes in the dimensionless acoustic power dissipation.

Summarizing the results so far, it can be concluded that for laminar oscillatory air flows the optimum jet pump performance is achieved between $0.2<K C_{\alpha}<0.5$ under the condition that $K C_{D}>0.5$. The combination of these two criteria imposes a limit on the maximum taper angle which maximizes the dimensionless pressure drop. Substitution of the two criteria, yields $\alpha<23^{\circ}$ in order to prevent overlapping of the range of optimum jet pump performance with the range where no pressure drop is measured. The optimum jet pump performance criterion does well coincide with the region where either right-sided or two-sided vortex shedding is observed (indicated with and in Fig. 3.2a). It is only in this optimum region that the measured dimensionless pressure drop will approach values as predicted by the quasi-steady model.

\subsubsection{Influence of waist curvature}

In the previous discussion where the influences of the jet pump taper angle and waist diameter were investigated, the radius of curvature at the jet pump waist was kept 
Table 3.4: Dimensions of jet pump geometries with varied radius of curvature $R_{c}$. Constant taper angle $\alpha=7^{\circ}$. Horizontal line separates cases at $\chi=0.15$.

\begin{tabular}{llll}
\hline No. & $R_{c}$ & $\chi$ & \\
\hline 14 & $0.0 \mathrm{~mm}$ & 0 & "sharp" \\
15 & $1.0 \mathrm{~mm}$ & 0.08 & \\
\hline 16 & $2.5 \mathrm{~mm}$ & 0.19 & \\
$r e f$ & $5.0 \mathrm{~mm}$ & 0.36 & "smooth" \\
17 & $7.0 \mathrm{~mm}$ & 0.48 & \\
18 & $10.0 \mathrm{~mm}$ & 0.65 & \\
\hline
\end{tabular}

constant. In steady flow theory, the minor loss coefficient for contraction is dependent on the dimensionless radius of curvature, ${ }^{58} \chi=R_{c} / D_{s}$. In order to maximize the time-averaged pressure drop while minimizing the acoustic power dissipation, it is desirable to minimize the contraction minor loss coefficient (following the quasi-steady approximation from Section 1.3.1) and as such, apply a smooth curvature at the jet pump waist. For a "smooth" contraction $(\chi>0.15)$ the theoretical minor loss coefficient is $K_{\text {con }}=0.04$ which increases up to $K_{\text {con }}=0.50$ for a sharp contraction. ${ }^{58}$ For all the jet pump geometries shown so far, a curvature of $\chi=0.36$ was applied. In the current section, the jet pump curvature will be varied to study both its effect on the jet pump performance as well as on the occurring flow phenomena.

Six different dimensionless curvatures have been investigated, ranging from $\chi=0$ to 0.65. Aside from the radius of curvature $R_{c}$, the jet pump geometry is equal to the reference geometry found in Table 3.1. An overview of the studied geometries with variable curvature is shown in Table 3.4.

\section{Jet pump performance}

When comparing the measured dimensionless pressure drop using these jet pump samples, it is found that an additional contribution to the Keulegan-Carpenter number is required to properly scale the results from different curvatures. Figure 3.6a shows the dimensionless pressure drop as a function of $K C_{D}$ for the geometries with varied curvature. The investigated curvatures are indicated by different symbols depicted in the corresponding legend. The crosses $(x)$ represent the reference geometry where $\chi=0.36$. The shape of the dimensionless pressure drop curve is found to be inversely proportional to the dimensionless curvature, especially for low values of $\chi$. For large curvatures, the effect on the dimensionless pressure drop becomes less apparent. Holman et al. studied the effect of edge curvature on the onset of a synthetic jet. ${ }^{68}$ A formation criterion of the form $K C_{D}>C \cdot(1+\chi)^{p}$ was derived based on the vorticity flux through the jet. The applicability of this criterion to jet pumps with variable curvature is investigated in the following. It is observed that especially the initial increase in $\Delta p_{2}^{*}$, corresponding to the onset of vortex shedding, can be properly scaled using $K C_{D} / C(1+\chi)^{p}$ with $p=4$. Given that the onset of vortex shedding for 

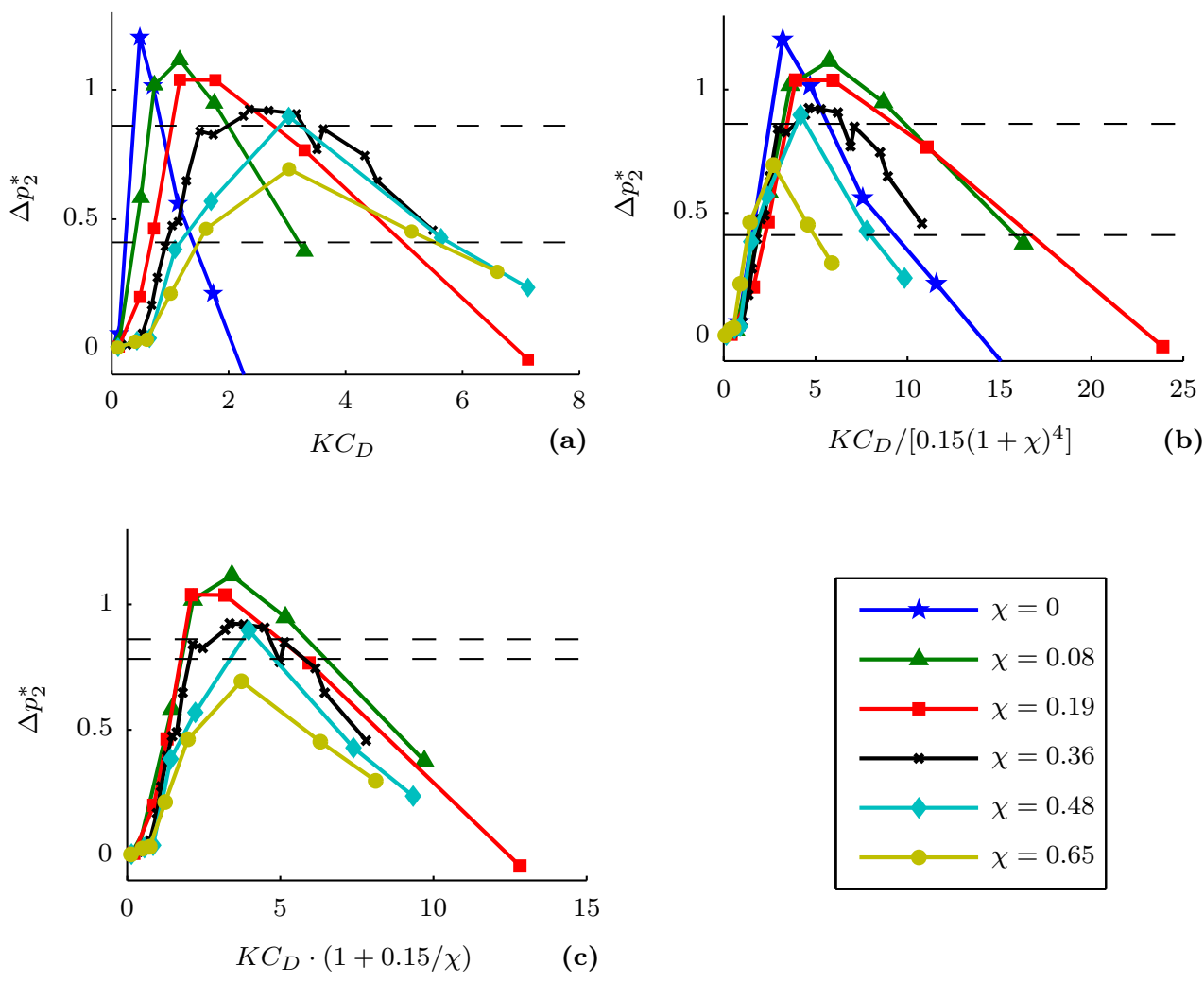

Figure 3.6: Dimensionless pressure drop using jet pump geometries with varied waist curvature as a function of three different scaling parameters: (a) $K C_{D}$, (b) $K C_{D} / 0.15(1+\chi)^{4}$ and (c) $K C_{D} \cdot(1+0.15 / \chi)$. The different symbols correspond to dimensionless curvatures ranging from $\chi=0$ to 0.65 . The dashed black lines indicate the upper and lower values from the quasi-steady approximation for the various values of $\chi$. 

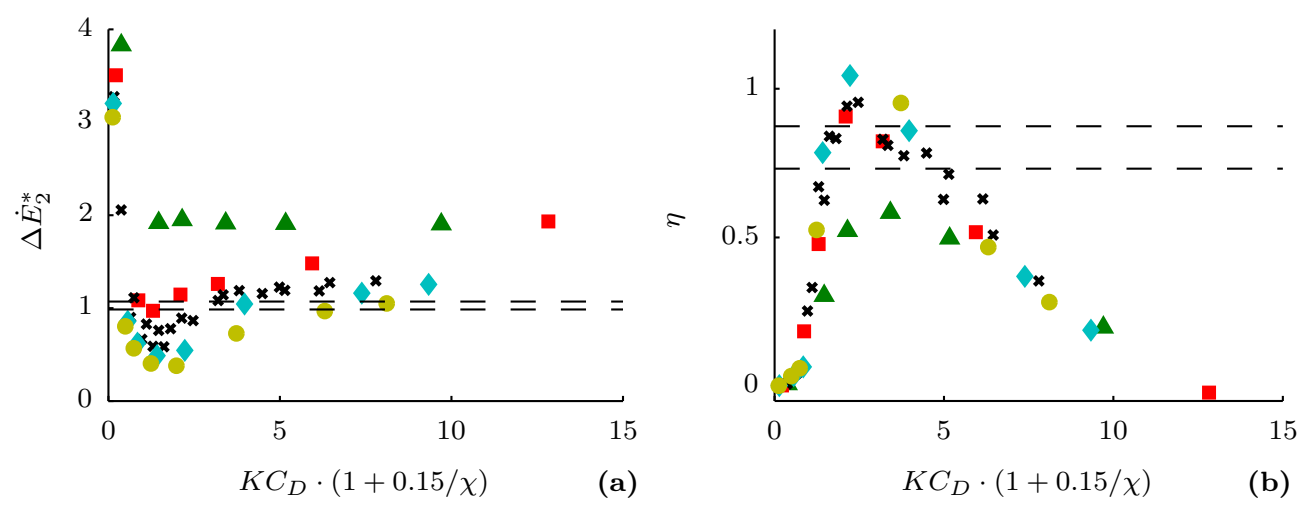

Figure 3.7: (a) Dimensionless acoustic power dissipation and (b) jet pump effectiveness using jet pump geometries with varied waist curvature and scaled using $K C_{D} \cdot(1+0.15 / \chi)$. The different symbols correspond to dimensionless curvatures ranging from $\chi=0.08$ to 0.65 as indicated in the legend in Fig. 3.6. The dashed black lines indicate the upper and lower values from the quasi-steady approximation for the various values of $\chi$.

a jet pump geometry with $\chi=0.36$ is found at $K C_{D}=0.5$, the additional scaling parameter $C=0.15$ can be estimated. Figure $3.6 \mathrm{~b}$ shows the dimensionless pressure drop as a function of the adjusted scaling parameter. While the initial increase in $\Delta p_{2}^{*}$ for the various curvatures all collapse to a single curve, the decay in $\Delta p_{2}^{*}$ due to the occurrence of flow separation cannot properly be accounted for by any scaling of the form $K C_{D} / C(1+\chi)^{p}$.

To overcome this, an alternative scaling strategy for $\chi$ is proposed of the form $K C_{D} \cdot(1+a / \chi)$. Using a least-squares approach, the fitting parameter $a=0.15$ is determined. Note that $0.15 / \chi$ represents the fraction of the dimensionless curvature with respect to a "smooth" contraction. Figure 3.6c shows again the dimensionless pressure drop data, but now plotted against $K C_{D} \cdot(1+0.15 / \chi)$. The case with no curvature $(\chi=0)$ does not appear in this figure as it will lead to infinite values on the horizontal axis. Both the maxima of the dimensionless pressure drop as well as the decay at higher amplitudes are well aligned using this alternative scaling approach.

What is remarkable is that the maximum measured dimensionless pressure drop decreases with increasing curvature, which is in contrast with the steady flow theory where the minor loss coefficient for contraction is expected to fall until $\chi>0.15$. The maximum achieved dimensionless pressure drop ranges from $\Delta p_{2}^{*}=1.20$ for a sharp contraction $(\chi=0)$ to $\Delta p_{2}^{*}=0.69$ for $\chi=0.65$ in an almost linear manner. Furthermore, the effect of curvature on the dimensionless pressure drop is not only reversed, but the variation is larger than what is predicted by the quasi-steady model (dashed lines). An explanation of this behavior is the effect of curvature on the expansion phase, which is typically neglected in a quasi-steady approximation of the jet pump performance. Not only will the minor loss coefficient for contraction at the jet pump waist decrease, but so does the minor loss coefficient for expansion. This was first experimentally determined by Smith under steady flow conditions. ${ }^{93} \mathrm{~A}$ decrease in 


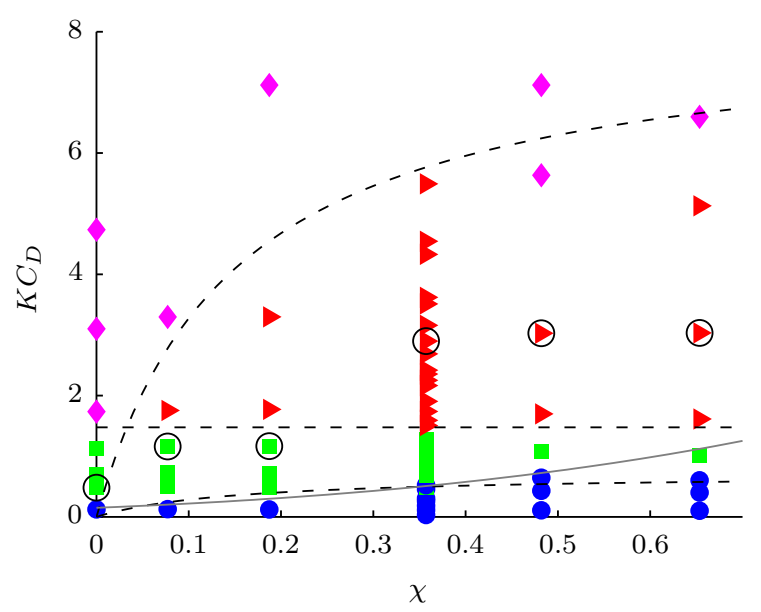

Figure 3.8: Distribution of the different flow regimes in the $\left(\chi, K C_{D}\right)$ space for jet pump geometries with varied curvature (Table 3.4) and a $7^{\circ}$ taper angle. Symbols are in accordance with Fig. 3.2 with the open circles showing the position where $\Delta p_{2}^{*}$ is maximum for each investigated curvature. The dashed lines represent the bounds of the different flow regimes determined according to Eq. 3.8-3.10 and the gray solid line represents the onset of vortex shedding according to Eq. 3.11.

the expansion minor loss coefficient at the jet pump waist will lead to a decrease in the dimensionless pressure drop according to Eq. 1.7.

Additionally, this is reflected in the measured dimensionless acoustic power dissipation, which is shown in Fig. 3.7a. The minimum achieved dimensionless acoustic power dissipation decreases more than six times between $\chi=0$ (not shown) and $\chi=0.65$. A similar effect has been reported when rounding the orifice of an synthetic jet. ${ }^{67}$ Combining the effect the curvature has on both the pressure drop and acoustic power dissipation using the jet pump effectiveness, shows that a higher dimensionless curvature leads to a better jet pump performance. This is depicted in Fig. 3.7b where the jet pump effectiveness for five investigated curvatures is shown.

\section{Flow regimes}

Similar to what was presented in Section 3.3.1, the observed jet pump behavior can be explained by studying the flow field and distinguishing between the four different flow regimes. Figure 3.8 shows the distribution of the flow regimes in the $\left(\chi, K C_{D}\right)$ space. The four flow regimes are represented by different symbols in accordance to the symbols used in Fig. 3.2. A clear influence of the dimensionless curvature is visible on the value of $K C_{D}$ where vortex shedding is first observed (transition from $\bullet$ to $\mathbf{\square}$ ) as well as on the value where flow separation is initiated (transition from $\boldsymbol{\downarrow}$ to $\downarrow$ ). The boundary between one-sided and two-sided vortex shedding (transition from $\mathbf{\square}$ to is not determined by the jet pump waist curvature at all. As the local curvature at the big opening is not changed, no dependency on $\chi$ is to be expected. 
By substituting the original defined flow regime bounds from Section 3.3.1 in the adjusted scaling parameter $K C_{D} \cdot(1+0.15 / \chi)$, the bounds between the flow regimes (indicated by symbols as used in Fig. 3.2 and Fig. 3.8) can be represented in the ( $\chi$, $\left.K C_{D}\right)$ space:

$$
\begin{aligned}
\text { - } \rightarrow: K C_{D} & =0.7\left(1+\frac{0.15}{\chi}\right)^{-1}, \\
\mathbf{\square} \rightarrow: K C_{D} & =0.15 \frac{R_{b}^{3}}{R_{s}^{3}}, \\
\text { \ } \rightarrow: K C_{D} & =\frac{1}{\alpha}\left(1+\frac{0.15}{\chi}\right)^{-1},
\end{aligned}
$$

where the first expression corresponds to $K C_{D}=0.5$, the second expression corresponds to $K C_{D, b}=0.15$ and the last expression corresponds to $K C_{\alpha}=0.7$ for the cases where $\chi=0.36$. The bounds are shown in Fig. 3.8 by the dashed lines. The bottom curve represents the initiation of vortex shedding (Eq. 3.8) and the upper curve the transition to flow separation (Eq. 3.10), both a function of $\chi$. The horizontal dashed line in Fig. 3.8 indicates the boundary between one-sided and two-sided vortex shedding.

Based on the data available the determined bounds do separate the flow regimes depicted in Fig. 3.8 well, although the defined onset of vortex shedding is subject to discussion. Given the fact that the initial increase in $\Delta p_{2}^{*}$ caused by the onset of vortex shedding was well described by adopting a scaling of the form proposed by Holman et al. ${ }^{68}$ a flow regime bound based on the latter could be more appropriate:

$$
\text { - } \rightarrow \mathbf{\square}: K C_{D}=0.15(1+\chi)^{4} .
$$

This is represented in Fig. 3.8 by a gray solid line and does indeed separate the two flow regimes. It should be noted that the data available is not sufficient to determine whether Eq. 3.8 or Eq. 3.11 is more appropriate.

Nevertheless, Eq. 3.11 predicts vortex shedding for $\chi=0$ at $K C_{D}>0.15$ which is also the formation criterion observed experimentally for a sharp edged synthetic jet. ${ }^{68}$ This exactly describes the difference in the transitional Keulegan-Carpenter number, observed in Section 3.3.1, between vortex shedding from the jet pump waist $\left(K C_{D, s}>0.5\right)$ and vortex shedding from the big jet pump opening $\left(K C_{D, b}>0.15\right)$. A lower dimensionless curvature leads to a lower value of $K C_{D}$ where vortex shedding is initiated. Consequently, vortex shedding at the sharp edged big opening will take place at a lower value of the local Keulegan-Carpenter number than the vortex shedding from the jet pump waist.

\subsection{Conclusions}

A CFD based parameter study is performed to investigate the influence of various geometric parameters on the performance of jet pumps for thermoacoustic applications. 
A total of 19 different jet pump geometries are considered and for each geometry a number of wave amplitudes are chosen, resulting in a total of 197 simulations. In correspondence with previous work, four flow regimes are distinguished and separated in a fixed variable space. ${ }^{73}$ This space spans the jet pump taper angle $\alpha$, the dimensionless curvature $\chi$ and the Keulegan-Carpenter number $K C_{D}$. At a certain value of $K C_{D}$, single-sided vortex propagation from the jet pump waist is initiated and the flow field becomes asymmetric between the left and right side of the jet pump. This onset is found at $K C_{D}=0.5$ for a dimensionless curvature of $\chi=0.36$. Two different expressions are proposed to account for the effect of $\chi$, but more data is required to decisively choose one of the two. At some point, flow separation inside the jet pump can be distinguished and vortices are shed from the jet pump waist in alternating directions leading to a flow field that is more symmetric. This transition is found to be strongly dependent on the jet pump taper angle and occurs when $K C_{D}>\frac{1}{\alpha}(1+0.15 / \chi)^{-1}$.

The amount of asymmetry in the flow field has a direct consequence on the measured time-averaged pressure drop. When no vortex shedding occurs, no dimensionless timeaveraged pressure drop is observed. Furthermore, as soon as the flow separation is observed, the dimensionless pressure drop has already decayed significantly compared to the maximum value. For all the performed simulations, the maximum jet pump effectiveness is observed when either single-sided or two-sided vortex propagation occurs. Consequently, the practical operation area of jet pumps with $D_{s} / \delta_{\nu} \gg 1$ and $R e<R e_{c}$ is bound between the onset of vortex shedding from the jet pump waist and the occurrence of flow separation. This can be used as a guideline for future jet pump design.

The design considerations presented in this chapter, are supposed to provide better insight into the validity of the quasi-steady approximation that is widely used for the design of jet pumps for thermoacoustic applications. The quasi-steady approximation is in most cases an idealized representation of the jet pump performance and is valid only in a small operation area. Outside this region, the actual jet pump performance is expected to be significantly lower and a correction using the presented results is advised.

Although the current parameter study represents a wide range of jet pump geometries, it is restricted to jet pumps with a single, linear tapered hole. As such, this work can serve as a basis for further jet pump geometry optimization in terms of effectiveness, compactness and robustness. Extensions to multiple holes or different shaped jet pump walls might affect the jet pump performance and the occurrence of flow separation. Furthermore, the influence of turbulence on the flow separation requires further attention. If the flow separation regime could be shifted to higher Keulegan-Carpenter numbers without a decrease in effectiveness, the operation area of a jet pump can be extended, greatly enhancing its robustness. 


\section{carran 4}

\section{Reducing flow separation in jet pumps}

Inside a jet pump, flow separation in the diverging flow direction can counteract the time-averaged pressure drop. In this chapter, the characteristics of flow separation in jet pumps are identified and coupled with the observed jet pump performance. Furthermore, it is shown that the onset of flow separation can be shifted to larger displacement amplitudes by designs that have a smoother transition between the small opening and the tapered surface of the jet pump. These design alterations also reduce the duration of separated flow, resulting in more effective and robust jet pumps. To make the proposed jet pump designs more compact without reducing their performance, the minimum big opening radius that can be implemented before the local minor losses have an influence on the jet pump performance is investigated. To validate the numerical results, they are compared with experimental results for one of the proposed jet pump designs.

\subsection{Introduction}

In the previous two chapters, Chapter 2-3, it has been shown that the accuracy of the quasi-steady approximation to predict a jet pump's performance is limited and that factors other than the expansion and contraction minor losses play an important part. ${ }^{73,85}$ Possibly, the most compelling example of this is the effect of the taper angle on the jet pump performance. Petculescu and Wilen did already show experimentally that a larger taper angle increases the minor losses for flow in the diverging jet pump direction. ${ }^{60}$ This will result in a significant performance drop due to the smaller timeaveraged pressure drop and the higher time-averaged acoustic power dissipation. They suggest that the dependency on the taper angle is caused by a shift in the axial point, and therewith the local radius, at which flow separation occurs. However, they could

Adapted from M. A. G. Timmer, J. P. Oosterhuis, S. Bühler, D. Wilcox, and T. H. van der Meer. "Characterization and reduction of flow separation in jet pumps for laminar oscillatory flows." $J$. Acoust. Soc. Am., 139(1):193-203, 2016. doi:10.1121/1.4939490 
not provide clear evidence of the existence of flow separation with their experimental setup.

In the presented numerical study (Ch. 2-3), the time-averaged velocity fields inside several jet pump geometries have been examined, and it was concluded from the axial streaming field that flow separation had indeed occurred. The separated flow was only observed above certain displacement amplitudes and its onset was found at lower displacement amplitudes for larger taper angles. The study also showed that a severe decrease in jet pump performance started at approximately the same displacement amplitude as the onset of flow separation. It was therefore suggested that the latter is the cause of the problematic performance drop observed above certain displacement amplitudes.

Next to axial streaming resulting from flow separation, it is necessary to examine the flow field in the vicinity of the separation in further detail. This will provide more insight into the influence of flow separation on the performance of jet pumps, which can subsequently be used to improve jet pump designs. In the following, a clear definition of separation in oscillatory flows is sought to serve as a basis for this investigation.

\subsubsection{Flow separation}

In an element with increasing cross-sectional area, such as the diverging direction of a jet pump, the boundary layer can separate due to the adverse pressure gradient overcoming the kinetic energy of the flow. ${ }^{95}$ In steady flow, the separation is often characterized by vanishing wall shear stresses and flow reversal at the most upstream point. Downstream of this point the flow is separated, resulting in recirculation and a wake of disturbed flow that generates minor losses. However, Despard and Miller have experimentally shown that flow reversal in an oscillatory flow occurs periodically at all surface points in the adverse pressure gradient regime, thus also upstream of the wake formation. ${ }^{96}$ They stress that the steady flow characterization of flow separation is thus unsuitable for oscillatory flow, and they propose to characterize flow separation with the surface point that first experiences flow reversal during the period of oscillation. This point can uniquely be determined and wake formation was found to invariably begin in the immediate neighborhood of this point. Simpson agreed that "it is too narrow a view" to characterize flow separation with vanishing surface shear stresses and flow reversal in oscillatory flow, since flow reversal can occur without the separation of the boundary layer. ${ }^{97} \mathrm{He}$ emphasizes that separation must mean the complete breakdown or "breakaway" of the boundary layer, which subsequently has a significant interaction with the bulk flow. It therefore seems necessary to extend the characterization of Despard and Miller with a measure that identifies the breakaway of the boundary layer. This could be done with the use of the time-averaged velocity fields inside the jet pump, as was shown in Chapter 2.

King and Smith performed experiments with a purely oscillatory flow in a diffuser to investigate flow separation and minor losses for different operating conditions and diffuser angles. ${ }^{62}$ They state that the minor losses are mainly due to random motions 
generated after the flow separates, and that it is therefore reasonable to assume that a longer duration of flow separation over a larger spatial extent results in larger minor losses. Due to the analogous adverse pressure gradient in a diffuser and a jet pump, their results show that one should aim to shorten the duration and spatial extent of flow separation in the diverging direction of a jet pump to increase its performance. King and Smith have shown that this can be accomplished by decreasing the taper angle of the geometry and by operating at either a higher Reynolds number for a given displacement amplitude or a lower displacement amplitude for a given Reynolds number. ${ }^{62}$

Schlichting had a more general view when he stated that the flow separation point is highly dependent on the adverse pressure gradient in the boundary layer, and that this pressure is imposed by the pressure of the bulk flow. ${ }^{92}$ This demonstrates that it is possible to control flow separation by geometrical variations that alter the adverse pressure gradient of the bulk flow. An example of this is the aforementioned decrease of the taper angle, but this might lead to undesirably long jet pumps. To increase the performance of jet pumps while keeping them compact, it therefore seems most effective to aim at reducing the adverse pressure gradient only in the immediate vicinity of the point of flow separation.

\subsubsection{Chapter outline}

In this chapter the flow separation in the adverse pressure gradient regime of jet pumps is investigated using a computational fluid dynamics model, which will be repeated briefly in Sec. 4.2. In Sec. 4.3 a measure to characterize flow separation in jet pumps is proposed and subsequently used to examine flow separation and its effect on the jet pump performance. In Sec. 4.4.1, jet pump designs that aim to suppress flow separation and increase the jet pump performance are investigated. Subsequently, partly due to the increased length of the proposed designs, the extent to which jet pumps can be made more compact without reducing their performance is investigated in Sec. 4.4.2. Finally, an experimental validation of the CFD model for one of the proposed jet pump designs is given in Sec. 4.4.3.

\subsection{Modeling}

The computational fluid dynamics model used in the present chapter is largely the same as the model used previously in Chapters 2-3, a complete description can be found in Sec. 2.2. In the current chapter, the experimental validation (Sec. 4.4.3) is performed at an acoustic frequency of $80 \mathrm{~Hz}$, while all other simulations are performed at an acoustic frequency of $20 \mathrm{~Hz}$. For the latter, this results in a time-step of $\Delta t=5 \cdot 10^{-5} \mathrm{~s}$. A schematic of the computational domain with the applied boundary conditions is given in Fig. 4.1. Note that the used jet pump geometry is different from the previous investigations, this will be further described in Sec. 4.3. 


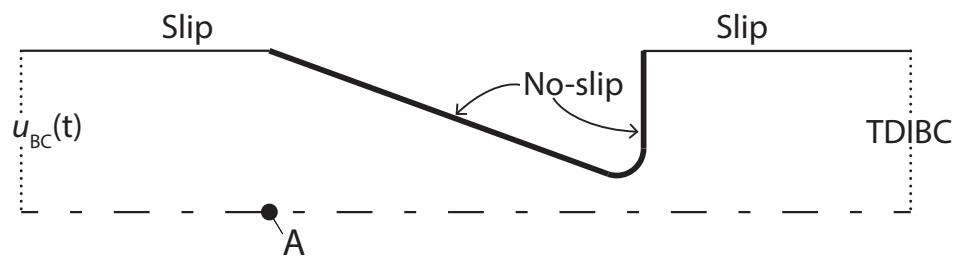

Figure 4.1: Schematic of the computational domain with the applied boundary conditions (not to scale). Point A represents the position where the time-averaged velocity $u_{2, L}$ is measured to detect flow separation (Sec. 4.3.1). Dotted lines at the left and right hand side depict the local boundary conditions and the bottom dashed line represents the symmetry axis.

Table 4.1: Dimensions of the reference geometry. Nomenclature according to Fig. 1.5.

\begin{tabular}{ll}
\hline$L_{J P}$ & $91.5 \mathrm{~mm}$ \\
$R_{0}$ & $30 \mathrm{~mm}$ \\
$R_{b}$ & $30 \mathrm{~mm}$ \\
$R_{s}$ & $7 \mathrm{~mm}$ \\
$R_{c}$ & $5 \mathrm{~mm}$ \\
$\alpha$ & $15^{\circ}$ \\
\hline
\end{tabular}

\subsubsection{Computational mesh}

The resolution of the computational mesh is similar to the meshes used in Ch. 2-3 and only briefly discussed here. Within a region of $50 \mathrm{~mm}$ from the jet pump, the mesh is unstructured and consists of triangular and quadrilateral elements with a maximum size of $1 \mathrm{~mm}$. A local refinement of $0.5 \mathrm{~mm}$ is used near the jet pump waist and at the transition from the no-slip to the slip boundary condition at the left hand side of the jet pump. Furthermore, an inflation layer is applied such that a minimum of 15 elements is present within a distance of $2 \pi \delta_{v}$ from the jet pump wall. In the remaining part of the domain, a structured mesh with only quadrilateral elements is constructed. In the radial direction the mesh size is fixed at $1 \mathrm{~mm}$, whereas in the axial direction the mesh size increases from $1 \mathrm{~mm}$ near the jet pump section up to $10 \mathrm{~mm}$ at the boundaries of the domain. The typical mesh size is 40000 nodes for the geometries presented in this chapter.

\subsubsection{Laminar flow}

The modeling of turbulence continues to be a major issue in predicting flow separation as was explained in Section 3.3. ${ }^{86}$ Turbulence models, such as the wide variety of lowReynolds number $k-\varepsilon$ models, fail to reproduce the near-wall flow characteristics in detail. ${ }^{90}$ Therefore, it is ensured in this work that the flow remains laminar within the entire computational domain so that no turbulence modeling is needed. For all results presented in this work, the acoustic Reynolds number in the jet pump waist is kept smaller than the critical Reynolds number. This verifies that the flow remains within 


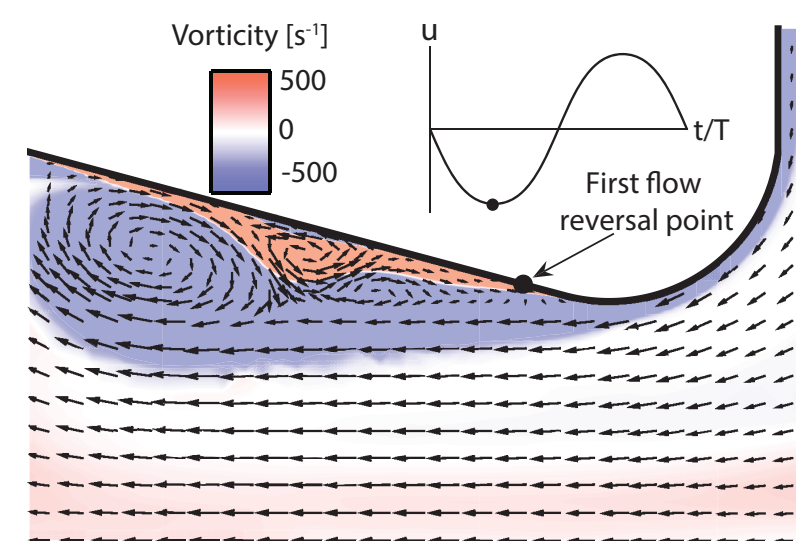

Figure 4.2: Vector plot of the velocity field for a section of the reference geometry where $t / T=0.25$ and $K C_{D}=3.5$. The colors depict the vorticity field.

the laminar regime, and thus no additional turbulence modeling is needed.

\subsection{Characterization of flow separation}

To characterize and investigate the separation of flow in the diverging direction of jet pumps, a reference geometry slightly different from the designs in Chapters $2-3$ is used (see Fig. 4.1). Table 4.1 lists the geometrical parameters for the reference geometry. Note that the big opening is eliminated, i.e. $R_{b}=R_{0}$, such that the tapered surface of the jet pump reaches the outer tube. This is done to independently investigate the separation of flow and the extent to which jet pumps can be made more compact (Sec. 4.4.2). This also negates the effect of vortex generation at the big opening from the flow separation analysis.

First, a measure to determine the breakaway of the boundary layer is specified using the numerical results of the reference geometry. This is followed with an analysis of the time and axial point at which flow reversal first occurs along the jet pump wall. Both results are then used to investigate the influence of flow separation on the jet pump performance.

\subsubsection{Boundary layer breakaway}

To characterize flow separation by the breakaway of the boundary layer, we focus on the half-period in which the bulk flow in the jet pump waist is moving leftward $(0<t / T<0.5)$. Here, a period of time $T$ is defined to start when the bulk flow velocity $u$ at the jet pump waist is zero, and the flow starts moving leftward. To identify the boundary layer breakaway, we first look at the flow in the vicinity of the jet pump wall. It is observed in all investigated cases that the flow in the boundary layer changes direction, while the bulk flow is still leftward. Thus, the boundary layer 
is leading the bulk flow in phase. An example where the reversed flow has already occurred is given in Fig. 4.2. Here, the velocity field at the end of the leftward acceleration $(t / T=0.25)$ is shown for $K C_{D}=3.5$. At this Keulegan-Carpenter number, the first flow reversal occurred at $t / T \approx 0.08$. This initially reversed flow has been observed to move rightward along the wall until it reached the contracting bulk flow at the jet pump waist. At this point, the reversed flow was prohibited from moving any further and it was forced to move downwards and recirculate into the bulk flow. This recirculation continues up to $t / T=0.5$, and induces a stream of vortices that propagate leftward with the bulk flow. These vortices and the resulting wake formation can be seen at the instant in time depicted in Fig. 4.2, and are found to be present for all cases investigated in this work.

Two distinct cases of wake formation as a function of displacement amplitude can be identified. For $K C_{D} \leq 1.7$, the induced vortices propagate only slightly towards the left, after which the bulk flow changes direction. The vorticity remains present for some time, but diminishes during the half-period of rightward bulk flow. This only results in a deviation from a purely oscillatory flow in the direct vicinity of the jet pump waist. In the rest of the flow field, no significant disturbances are caused by this vorticity. For $K C_{D}>1.7$, the vorticity does not diminish completely during the rightward bulk flow. This causes time-averaged streaming inside the jet pump, significantly disturbing the purely sinusoidal flow. The latter case is characterized as flow separation due to the large disturbance of the bulk flow caused by the breakaway of the boundary layer. It can be identified by a negative time-averaged velocity at the symmetry axis and a positive time-averaged velocity inside the rest of the jet pump, as shown in Chapter 2. The transition between the two cases occurs when the vorticity induced by the flow reversal remains present during the rightward acceleration of the bulk flow $(0.5<t / T<0.75)$. If the vorticity is still present after $t / T=0.75$, the flow at the symmetry axis is found to remain predominantly negative and flow separation has occurred. As a measure for the latter, we state that flow separation has occurred if

$$
\frac{u_{2, L}}{\left|u_{1, F F}\right|} \leq-1 \text {, }
$$

where $u_{2, L}$ is the time-averaged axial velocity at the point just left of the big jet pump opening, as depicted by point A in Fig. 4.1. $\left|u_{1, F F}\right|$ is the amplitude of the far field oscillatory flow at the left hand side of the jet pump. For undisturbed oscillatory flow, this velocity amplitude is approximately equal in the entire outer tube to the left of the jet pump. Equation 4.1 therefore states that flow separation has occurred if there is a negative streaming velocity at the symmetry axis that is bigger than the local amplitude of the oscillatory flow. It is found that the ratio of Eq. 4.1 is slightly positive if no flow separation occurs and is significantly less than -1 if flow separation does occur. It therefore seems to be an appropriate measure to identify the breakaway of the boundary layer and the resulting flow separation. 


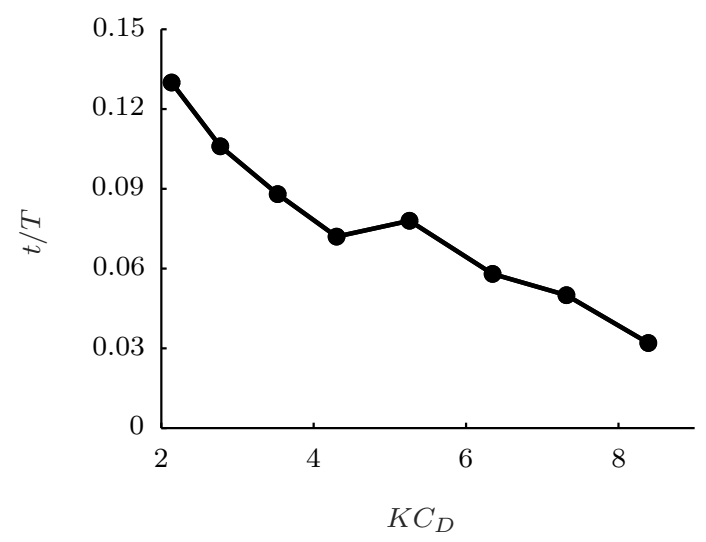

Figure 4.3: Time at which flow reversal was first found as a function of $K C_{D}$. Only the cases with flow separation for the reference geometry are depicted.

\subsubsection{First flow reversal}

So far, it has been shown that flow reversal along the jet pump wall occurs during leftward bulk flow. Despard and Miller state that the time and axial point at which this flow reversal first occurs is important to characterize flow separation. ${ }^{96}$ Therefore, an algorithm is written that searches for the point along the jet pump wall where the flow changes sign during negative bulk flow $(0<t / T<0.5)$. The axial point and time at which this occurs are averaged over the last five wave periods.

It is found that the axial point of first flow reversal is situated $1.9 \mathrm{~mm}$ left of the jet pump waist, where the local radius is $7.4 \mathrm{~mm}$ (see Fig. 4.2). This result is found to be independent of the displacement amplitude and oscillation frequency, which was also reported by Despard and Miller in their experimental work. ${ }^{96}$ The leftward wake formation starts in the immediate vicinity of the first flow reversal point, although this is hard to clarify for the reference geometry as this point is close to the jet pump waist. This will become clearer in Sec. 4.4.1, where geometries are presented that shift the first flow reversal point away from the jet pump waist.

The time at which flow reversal first occurs is depicted in Fig. 4.3 as a function of $K C_{D}$ for the cases with flow separation. For increasing displacement amplitude, the first occurrence of flow reversal shifts to earlier on in the period. Following King and Smith, it is therefore reasonable to state that the longer duration of flow separation for increasing displacement amplitudes generates more minor losses in the leftward direction. ${ }^{62}$ In turn, this will reduce the time-averaged pressure drop across the jet pump when compared with the situation where no flow separation would occur. To investigate the impact of reducing the time of first flow reversal on the jet pump performance, the dimensionless pressure drop in the flow separation regime is examined in the following section. 


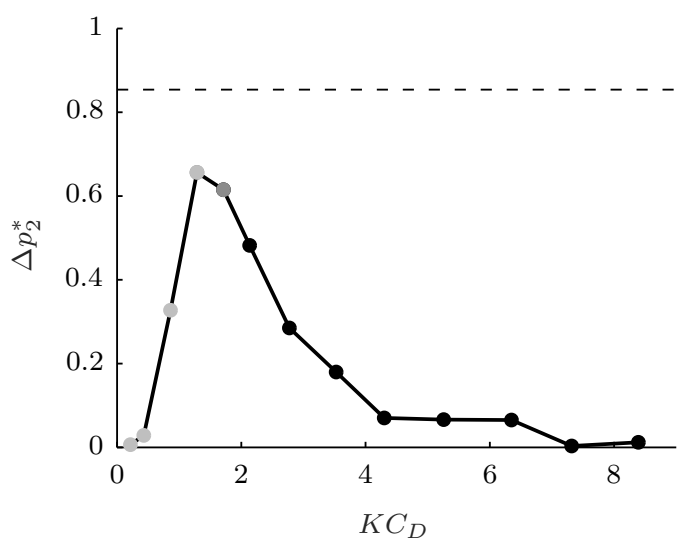

Figure 4.4: Dimensionless pressure drop as a function of $K C_{D}$ for the reference geometry. Three flow regimes are shown; no flow separation (light gray markers), partial flow separation (dark gray marker), and full flow separation (black markers). The dashed black line represents the quasi-steady approximation.

\subsubsection{Effect of flow separation on performance}

Now that flow separation has been characterized in terms of the breakaway of the boundary layer and flow reversal, its influence on the jet pump performance can be investigated. The dimensionless pressure drop as a function of $K C_{D}$ for the reference geometry is depicted in Fig. 4.4. For $K C_{D}<0.5$, there is no significant dimensionless pressure drop. Thereafter it starts increasing rapidly for larger displacement amplitudes, up to a maximum at $K C_{D}=1.3$. After this maximum, a severe decay in the dimensionless pressure drop for increasing displacement amplitudes occurs. Therefore, there is only a small range of displacement amplitudes in which the jet pump effectively produces the required pressure drop to cancel Gedeon streaming. Moreover, even if the jet pump is operated around the point of maximum performance, there is a large sensitivity of the performance on small changes in the operating conditions. To suppress Gedeon streaming, it can therefore be difficult to induce the correct amount of time-averaged pressure drop with this jet pump design. In the search for more robust jet pump designs, which do not have a small range of effective performance, it is important to understand why the dimensionless pressure drop reduces so severely above certain displacement amplitudes.

The black markers in Fig. 4.4 depict the dimensionless pressure drop of the cases where flow separation according to Eq. 4.1 was found. All cases are situated in the declining part of the curve of the dimensionless pressure drop, i.e. after the maximum at $K C_{D}=1.3$. This shows that there is a direct relation between flow separation and a decrease in jet pump performance. For increasing displacement amplitude, the negative effect of flow separation on the dimensionless pressure drop is increasing. As described in the previous section, this can be attributed to flow reversal occurring earlier on in the period (see Fig. 4.3), which induces more minor losses that reduce the dimensionless pressure drop. 


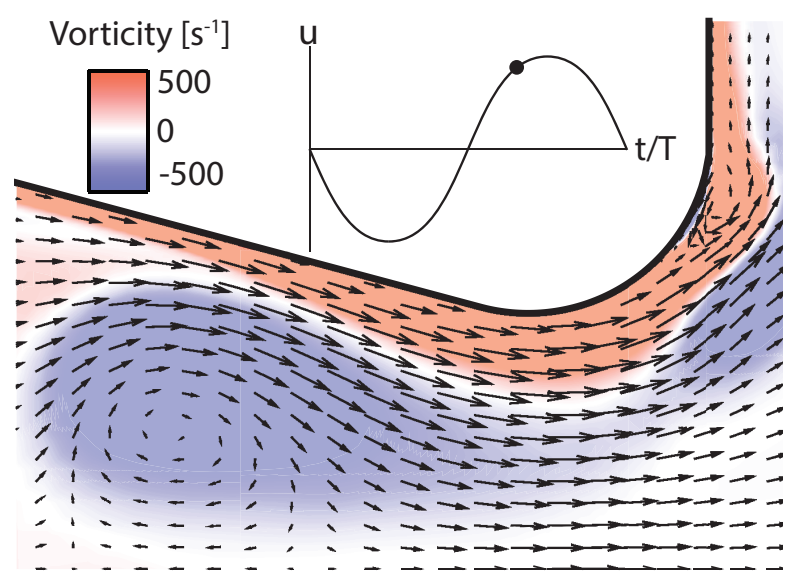

Figure 4.5: Vector plot of the velocity field for a section of the reference geometry where $t / T=0.65$ and $K C_{D}=1.7$. The colors depict the vorticity field.

The light gray markers in Fig. 4.4 show the cases were no flow separation is present and the dimensionless pressure drop rises with increasing $K C_{D}$. The dimensionless pressure drop does not reach the value of the quasi-steady approximation (dashed black line). This can be attributed to flow separation, recalling that only expansion and contraction minor losses at the jet pump openings are included in the quasisteady approximation. The onset of flow separation occurs at $K C_{D}=2.1$, which is such a low Keulegan-Carpenter number that the dimensionless pressure drop can not increase up to the value that it could without flow separation. To acquire a higher maximum dimensionless pressure drop, the onset of flow separation should therefore be shifted to a higher displacement amplitude, as attempted in Sec. 4.4.1.

So far, the decreasing dimensionless pressure drop has been attributed to flow separation, but why the dimensionless pressure drop decreases before the onset of flow separation according to Eq. 4.1 has not been explained. First of all, it should be clear that a decay of the dimensionless pressure drop does not represent a decay of the time-averaged pressure drop; it means that the latter increases in a manner less than quadratic. We thus seek to explain why the time-averaged pressure drop changes from increasing more than quadratically (light gray markers in Fig. 4.4) to less than quadratically (dark gray marker in Fig. 4.4). The velocity field of the first case that experiences a decrease in the dimensionless pressure drop is shown during the rightward bulk flow in Fig. 4.5. At the depicted instant in time $(t / T=0.65)$, the generation of the rightward expansion minor losses has just started. This can be seen by the flow reversal and vorticity along the right hand side of the jet pump wall. Additionally, the vorticity induced during the leftward bulk flow is still present just to the left of the jet pump waist. This vorticity will diminish before $t / T=0.75$, and will not cause time-averaged streaming inside the jet pump. However, it does prevail up to the time where the generation of the expansion minor losses in the rightward direction starts. Therefore, the vorticity reduces the strength of the rightward bulk flow during this period, and therewith the minor losses in the rightward direction 
that are predominantly responsible for the time-averaged pressure drop across the jet pump. In contrast, for the cases with an increasing dimensionless pressure drop $\left(K C_{D} \leq 1.3\right)$, the vorticity is completely diminished during the time at which the expansion minor losses start. This shows that the interaction between the two causes a decrease in the dimensionless pressure drop for displacement amplitudes even lower than those where flow separation is present. This situation is referred to as partial flow separation, since there is a short interaction of the induced vorticity with the bulk flow, but no time-averaged streaming inside the jet pump occurs.

The negative effect on the time-averaged pressure drop for the partial flow separation is also present for the case of full flow separation. Due to the non-diminishing vorticity and resulting negative streaming at the symmetry axis, the effect will even be larger. Therefore, next to the wake formation that increases the minor losses in the leftward direction, flow separation also decreases the minor losses in the rightward direction. This shows another incentive to reduce flow separation in an effort to increase the performance of jet pumps.

\subsection{Reduction of flow separation}

In the preceding section it is shown that the point of first flow reversal is close to the jet pump waist for the reference geometry. In an effort to reduce the negative effects of flow separation on the jet pump performance, we propose design alterations that reduce the adverse pressure gradient in the vicinity of this point.

\subsubsection{Increased transition length}

A reduced adverse pressure gradient near the point of first flow reversal is ensured by creating a longer and smoother transition from the jet pump waist to the tapered surface. For the reference geometry, this transition is done by a circle trajectory with the same radius as the radius of curvature of the small opening. For the adjusted designs, the transition radius $R_{t}$ is increased while keeping the radius of curvature $R_{c}$ of the small jet pump opening constant. An example of such a design is depicted in Fig. 4.6, where $R_{t}=28 \mathrm{~mm}, L_{J P}=94.5 \mathrm{~mm}$, and the other parameters are the same as the reference geometry. When compared with the reference geometry $\left(R_{t}=5 \mathrm{~mm}\right.$ and $L_{J P}=91.5 \mathrm{~mm}$ ), this design has an increased length $\Delta L_{J P}$ of $3 \mathrm{~mm}$ due to the elongated transition length. By further varying $R_{t}$, different geometries are designed that are characterized by the extra transition length $\Delta L_{J P}$ they introduce with respect to the reference case. Six different geometries are examined: $\Delta L_{J P}=3 \mathrm{~mm}, 5 \mathrm{~mm}$, $10 \mathrm{~mm}, 20 \mathrm{~mm}, 30 \mathrm{~mm}$ and $50 \mathrm{~mm}$.

The performance of the adjusted jet pump geometries and the reference geometry is depicted in Fig. 4.7 in terms of the (a) dimensionless pressure drop and (b) acoustic power dissipation. All geometries show a very similar increase of the dimensionless pressure drop for increasing displacement amplitude up to $K C_{D}=1.3$. At this 


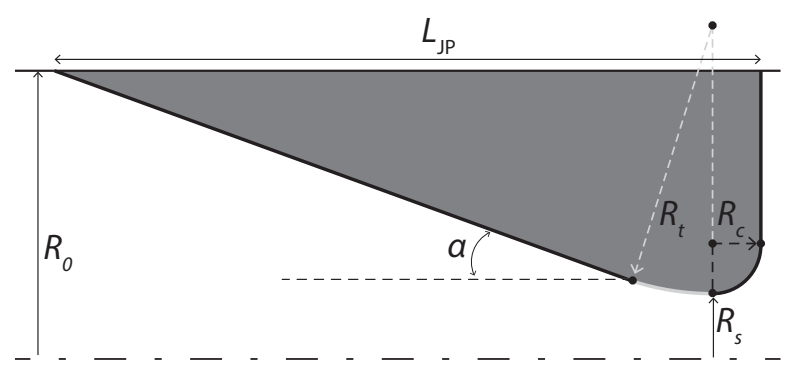

Figure 4.6: Axisymmetric representation of the adjusted jet pump with parameters that define the geometry. Bottom dashed line indicates center line, top solid line indicates outer tube wall. $R_{t}$ is varied to acquire different geometries.

Keulegan-Carpenter number, the displacement amplitude up to which no flow separation is present (light gray markers) is reached for the reference geometry. Here a maximum dimensionless pressure drop of $\Delta p_{2}^{*}=0.66$ is acquired. For the cases with an increased transition length, the region of no flow separation (light gray markers) is extended to a larger displacement amplitude compared with the reference geometry. Therefore, the dimensionless pressure drop continues to increase, resulting in an improvement of the maximum dimensionless pressure drop up to $\Delta p_{2}^{*}=0.91$ for $\Delta L_{J P}=50 \mathrm{~mm}$. It is worth noting that for the geometries with the largest transition lengths, the flow separation is postponed to such a displacement amplitude that the dimensionless pressure drop even reaches the value predicted by the quasi-steady approximation (dashed black line). Eventually, just as for the reference geometry, the dimensionless pressure drop decreases again for all geometries with increasing displacement amplitude. However, for a larger $\Delta L_{J P}$ the decrease of the dimensionless pressure drop is significantly reduced compared with a smaller transition length. This results in a broader range of displacement amplitudes where the dimensionless pressure drop is near its maximum for increasing $\Delta L_{J P}$. Therefore, a larger transition length shows a positive influence on the maximum dimensionless pressure drop as well as the robustness of the jet pump.

In Fig. 4.7a, it can be seen that the initial increase of the dimensionless pressure drop up to a maximum occurs for all geometries in the regime where no flow separation is present (light gray markers). The regime of partial flow separation (dark gray markers) is first found at displacement amplitudes just above the maximum dimensionless pressure drop. This is followed by full flow separation (black markers) and a decrease of the dimensionless pressure drop. These results show that for increased transition lengths, the different flow regimes occur at the same characteristic points of the dimensionless pressure drop curve as for the reference geometry. This confirms the proposed characterization of flow separation and its influence on the jet pump performance.

So far, we have only examined the dimensionless pressure drop, but the dimensionless acoustic power dissipation is also an important measure to quantify the jet pump 


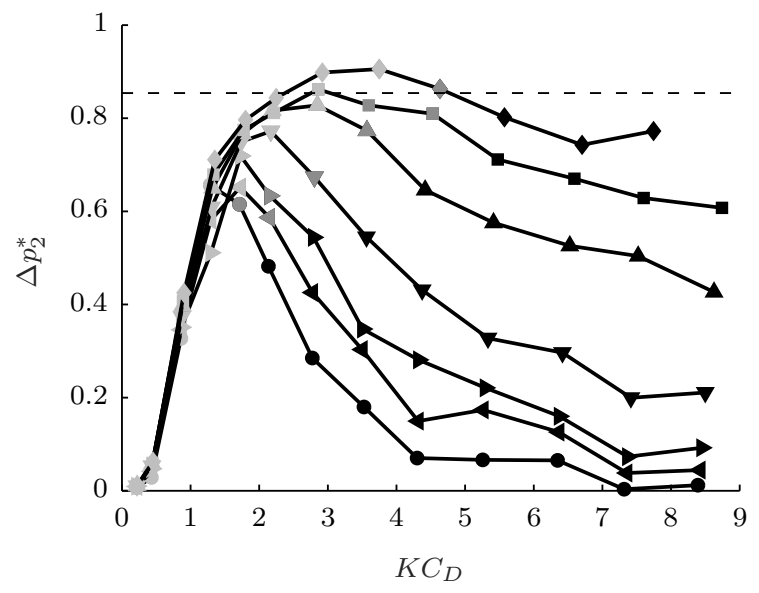

(a)

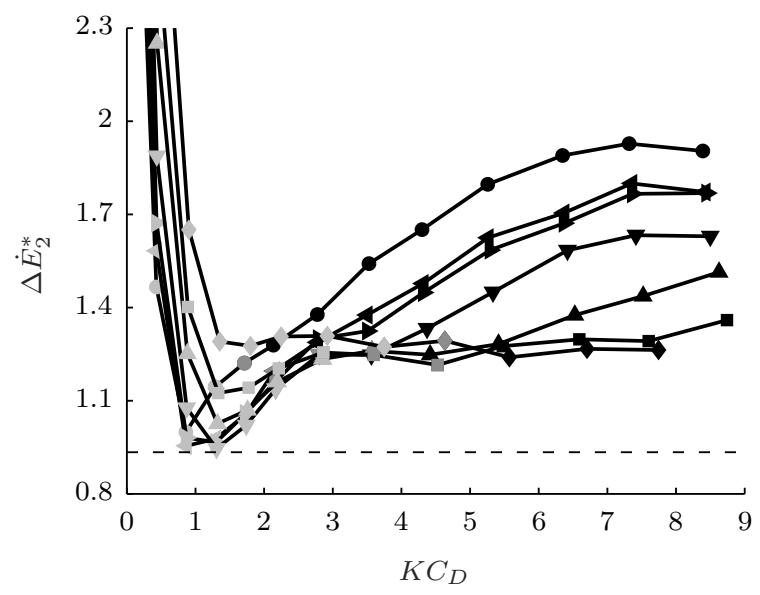

(b)

Figure 4.7: (a) Dimensionless pressure drop and (b) acoustic power dissipation as a function of $K C_{D}$ for the transition lengths: $\Delta L_{J P}=0 \mathrm{~mm}\left(\bullet\right.$, reference), $\Delta L_{J P}=3 \mathrm{~mm}(\bullet), \Delta L_{J P}=5 \mathrm{~mm}$ $(\bullet), \Delta L_{J P}=10 \mathrm{~mm}(\boldsymbol{\nabla}), \Delta L_{J P}=20 \mathrm{~mm}(\boldsymbol{\Delta}), \Delta L_{J P}=30 \mathrm{~mm}(\boldsymbol{\nabla})$, and $\Delta L_{J P}=50 \mathrm{~mm}(\bullet)$. Three flow regimes are depicted: no flow separation (light gray markers), partial flow separation (dark gray markers), and full flow separation (black markers). The dashed black line represents the quasi-steady approximation. 


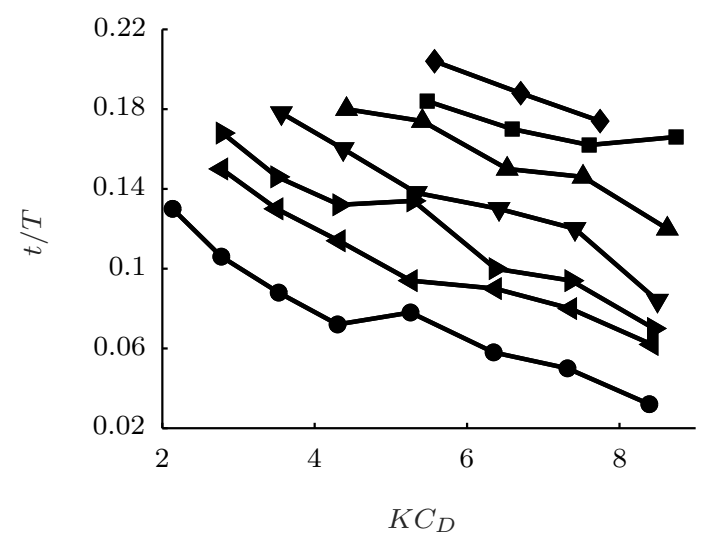

Figure 4.8: Time at which flow reversal was first found as a function of $K C_{D}$. Only the cases with flow separation are depicted. Symbols indicate the transition lengths: $\Delta L_{J P}=0 \mathrm{~mm}(\bullet$, reference), $\Delta L_{J P}=3 \mathrm{~mm}(\boldsymbol{\triangleleft}), \Delta L_{J P}=5 \mathrm{~mm}(\boldsymbol{\vee}), \Delta L_{J P}=10 \mathrm{~mm}(\boldsymbol{\nabla}), \Delta L_{J P}=20 \mathrm{~mm}(\boldsymbol{\Delta})$, $\Delta L_{J P}=30 \mathrm{~mm}(\boldsymbol{\square})$, and $\Delta L_{J P}=50 \mathrm{~mm}(\bullet)$.

performance. An effective jet pump will have a high pressure drop at the cost of a low acoustic power dissipation. In Fig. $4.7 \mathrm{~b}$ it can be seen that all geometries have an asymptotically high $\Delta \dot{E}_{2}^{*}$ for displacement amplitudes that approach zero. This is caused by the scaling of the acoustic power dissipation with the velocity amplitude cubed, which is very small for these displacement amplitudes. The dimensionless acoustic power dissipation then drops quickly with increasing displacement amplitude. In the regime without flow separation (light gray markers), the cases with a larger transition length have more acoustic power dissipation. This can be explained by the increased jet pump length in the vicinity of the jet pump waist, where the velocity amplitude is the largest and therewith the most viscous losses occur. For increasing $K C_{D}$, flow separation (black markers) sets in causing the dimensionless acoustic power dissipation to increase significantly, especially for the cases with the smallest $\Delta L_{J P}$. As a result of this, a larger transition length is favorable for high displacement amplitudes in the flow separation regime, due to the reduced acoustic power dissipation. This can be explained by the reduction of the minor losses generated by flow separation compared with a case that has a smaller transition length. This same reason also ensured a larger dimensionless pressure drop for a higher $\Delta L_{J P}$ in the flow separation regime (black markers in Fig. 4.7a).

To investigate why the minor losses induced by flow separation are reduced for increased transition lengths, the first point of flow reversal is identified for the cases where flow separation occurs. The time at which the first flow reversal is found is depicted in Fig. 4.8 as a function of $K C_{D}$. All geometries show a decrease in the time of first flow reversal with increasing displacement amplitude, which will cause more minor losses in the leftward direction. This concurs with the reduction of the dimensionless pressure drop and the increase of the dimensionless acoustic power dissipation for an increasing displacement amplitude. For all displacement amplitudes in the flow separation regime, an increase in $\Delta L_{J P}$ leads to a delay in the time of first flow rever- 


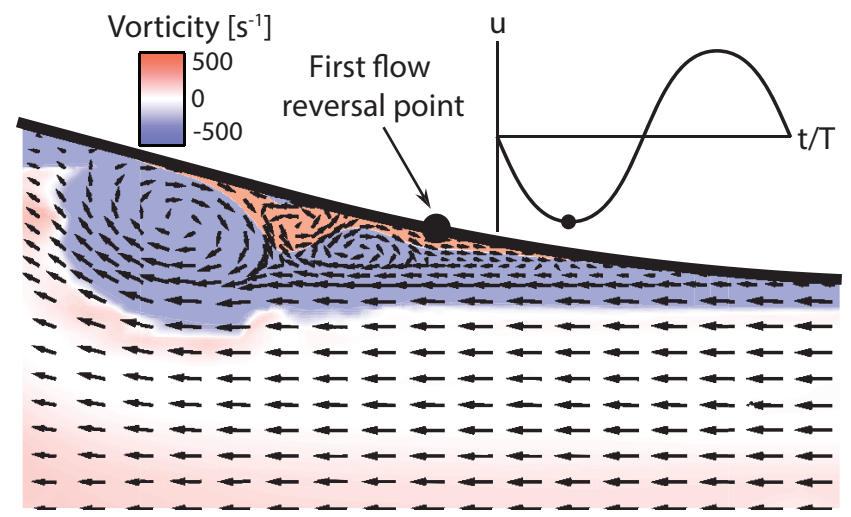

Figure 4.9: Vector plot of the velocity field in the vicinity of the jet pump waist for the geometry with $\Delta L_{J P}=10 \mathrm{~mm}$, where $t / T=0.25$ and $K C_{D}=7.4$. The colors depict the vorticity field.

sal. Therefore, by increasing the transition length, the minor losses generated by the wake formation of flow separation are reduced. This explains why the dimensionless pressure drop is higher and the dimensionless acoustic power dissipation is lower for larger transition lengths in the flow separation regime. Next to that, it can be seen that the onset of flow separation occurs at a higher displacement amplitude for a larger transition length. This ensures that the maximum dimensionless pressure drop is increased and shifted to higher values of $K C_{D}$, as can be seen in Fig. 4.7a.

The spatial point along the wall where flow reversal first occurs is found to shift away from the jet pump waist for an increasing transition length. For all cases, the leftward wake formation caused by the reversed flow was found to begin in the immediate vicinity of this point. An example of this is shown in Fig. 4.9, for the case where $\Delta L_{J P}=10 \mathrm{~mm}$ and $t / T=0.25$. Here a leftward propagating wake is formed from the point of first flow reversal, while the flow to the right of this point remains calm and does not generate any significant minor losses.

As previously mentioned, the point of first flow reversal is shifted away from the jet pump waist. However, due to the different slopes of the geometries in the vicinity of the jet pump waist, it is more meaningful to look at the local radius $R_{\text {sep }}$ at which flow reversal first occurs. For a specific displacement amplitude and frequency, the local radius will determine the local fluid velocity. This will in turn determine the magnitude of the minor losses of the leftward wake formation. Figure 4.10 depicts $R_{\text {sep }}$ as a function of $\Delta L_{J P}$, where the error bars indicate the standard deviation of $R_{s e p}$ for different displacement amplitudes at a given $\Delta L_{J P}$. For the reference case $R_{s e p}=$ $7.4 \mathrm{~mm}$, which is very close to the radius at the jet pump waist $\left(R_{s}=7.0 \mathrm{~mm}\right)$, and hence large minor losses are generated. By elongating the transition length, the local radius of flow reversal can be increased to $R_{s e p} \approx 8.5 \mathrm{~mm}$ for $\Delta L_{J P}=5 \mathrm{~mm}$. Further increasing the transition length has no significant effect on $R_{\text {sep }}$ for the geometries that are investigated in this work. Nevertheless, the increase of $R_{s e p}$ will reduce the minor losses generated by the wake formation when compared with the reference geometry, therewith increasing the jet pump performance. 


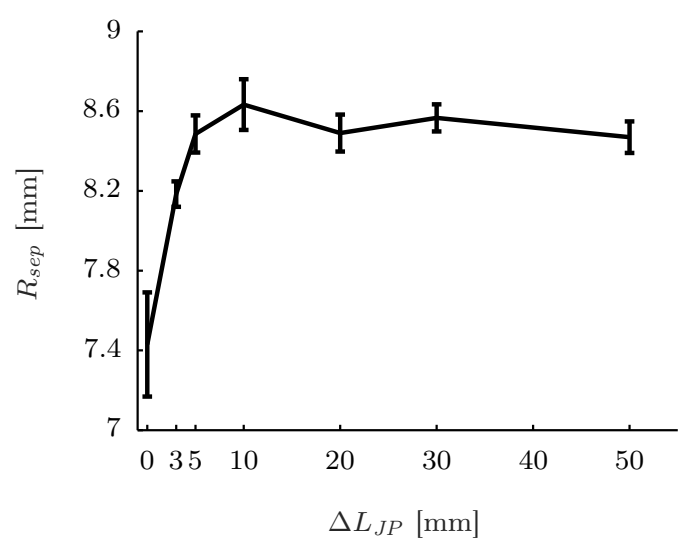

Figure 4.10: Local radius $R_{s e p}$ at which flow reversal is first found as a function of $\Delta L_{J P}$. Error bars indicate the standard deviation of $R_{\text {sep }}$ for different displacement amplitudes at a given $\Delta L_{J P}$.

\subsubsection{Compact designs}

In Sec. 4.4.1 it is shown that by increasing the transition length between the small jet pump opening and the tapered surface, both the effectiveness and robustness of the jet pump are significantly increased. The disadvantage of improving the performance in this manner is the increased jet pump length, which prohibits the design of compact thermoacoustic devices. So far we have investigated designs that have a tapered surface up to the outer tube radius (Fig. 4.6), while jet pumps can be made more compact by cutting the tapered surface at a specific radius $R_{b}$ (as shown in Fig. 1.5). According to the quasi-steady approximation, the minor losses generated at the big opening will decrease the jet pump performance. However, they are estimated to be a factor $\left(R_{s} / R_{b}\right)^{4}$ less than those at the small opening. Therefore, the extent to which the radius of the big opening, and therewith the total jet pump length, can be reduced before a significant performance drop occurs is investigated. This is done for the geometry with $\Delta L_{J P}=20 \mathrm{~mm}$ as this design is not much longer than the reference geometry, but still shows a great performance improvement (see Fig. 4.7). The total length of the geometry with $\Delta L_{J P}=20 \mathrm{~mm}$ is $L_{J P}=111.5 \mathrm{~mm}$. The performance of six geometries with a reduced length are compared with this geometry, namely: $L_{J P}=63.5 \mathrm{~mm}, 49.5 \mathrm{~mm}, 45.5 \mathrm{~mm}, 40.5 \mathrm{~mm}, 35.5 \mathrm{~mm}$ and $25.5 \mathrm{~mm}$. The corresponding big hole radii are $R_{b}=17.1 \mathrm{~mm}, 13.4 \mathrm{~mm}, 12.3 \mathrm{~mm}, 11.1 \mathrm{~mm}, 10.0 \mathrm{~mm}$ and $8.3 \mathrm{~mm}$, respectively. Note that the radius of the small opening, $R_{s}$, is $7 \mathrm{~mm}$ for all geometries.

In Fig. 4.11 the dimensionless pressure drop is shown as a function of $R_{b}$ for several Keulegan-Carpenter numbers. The jet pump performance is depicted for values of $K C_{D}$ in the no flow separation regime $\left(K C_{D}=1.3\right.$ and $\left.K C_{D}=2.8\right)$, the partial flow separation regime $\left(K C_{D}=3.6\right)$, and the full flow separation regime $\left(K C_{D}=5.4\right)$. It can be seen that there is no difference in the trend for the various values of $K C_{D}$. Therefore, reducing the big hole radius has a similar effect in each flow regime. At all Keulegan-Carpenter numbers, no performance drop is present for a small reduction 


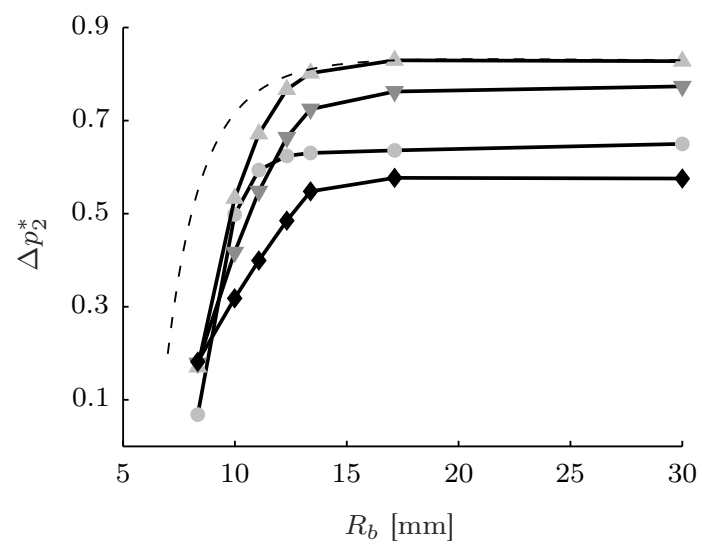

Figure 4.11: Dimensionless pressure drop as a function of $R_{b}$. Lines indicate a constant $K C_{D}$ for the values: $K C_{D}=1.3(\odot), K C_{D}=2.8(\Delta), K C_{D}=3.6(\nabla)$, and $K C_{D}=5.4(\diamond)$. Dashed line represents the quasi-steady approximation.

of the big hole radius. For these large radii, the quasi-steady approximation is an excellent approximation of the maximum pressure drop (at $K C_{D}=2.8$ ). For $R_{b} \lesssim$ $13 \mathrm{~mm}$, the minor losses generated at the big hole become too large, resulting in a significant reduction of $\Delta p_{2}^{*}$ (see Fig. 4.11) and increase of $\Delta \dot{E}_{2}^{*}$. When using the expansion minor loss coefficient for steady flow (see Eq. 1.6 on page 8), the quasisteady approximation underestimates the performance drop due to the big hole size reduction. The transition in performance at $R_{b} \lesssim 13 \mathrm{~mm}$ was found at the same big hole radius for the reference geometry $\left(\Delta L_{J P}=0 \mathrm{~mm}\right)$, and can therefore be used as a general result for all geometries presented in this work.

Despite the reduced performance for a geometry with a big hole radius that approaches the small hole radius, the length of jet pumps can still be significantly decreased. As an example, for the cases with $\Delta L_{J P}=20 \mathrm{~mm}$ (Fig. 4.11), the geometry with $L_{J P}=49.5 \mathrm{~mm}$ has the same performance as the geometry with $L_{J P}=111.5 \mathrm{~mm}$. This shows that the jet pumps, as proposed in section 4.4.1, can be made much more compact without reducing their performance.

\subsubsection{Experimental validation}

To validate the numerical results presented in this work, the jet pump design with $\Delta L_{J P}=20 \mathrm{~mm}, R_{b}=13.4 \mathrm{~mm}$, and $L_{J P}=49.5 \mathrm{~mm}$ is manufactured using a $3 \mathrm{D}$ printing method. The performance of the jet pump is investigated in the experimental setup that is previously used by Aben. ${ }^{75}$ The jet pump is situated in a tube with two pressure sensors on either side of the jet pump. An acoustic wave with a frequency of $80 \mathrm{~Hz}$ is generated by a loudspeaker. At each displacement amplitude, the mean pressure and the pressure amplitude are determined by averaging over $60 \mathrm{~s}$. For accuracy, the data where the standard deviation of the pressure is larger than $10 \%$ of the mean pressure has been left out. From the remaining pressure data in 

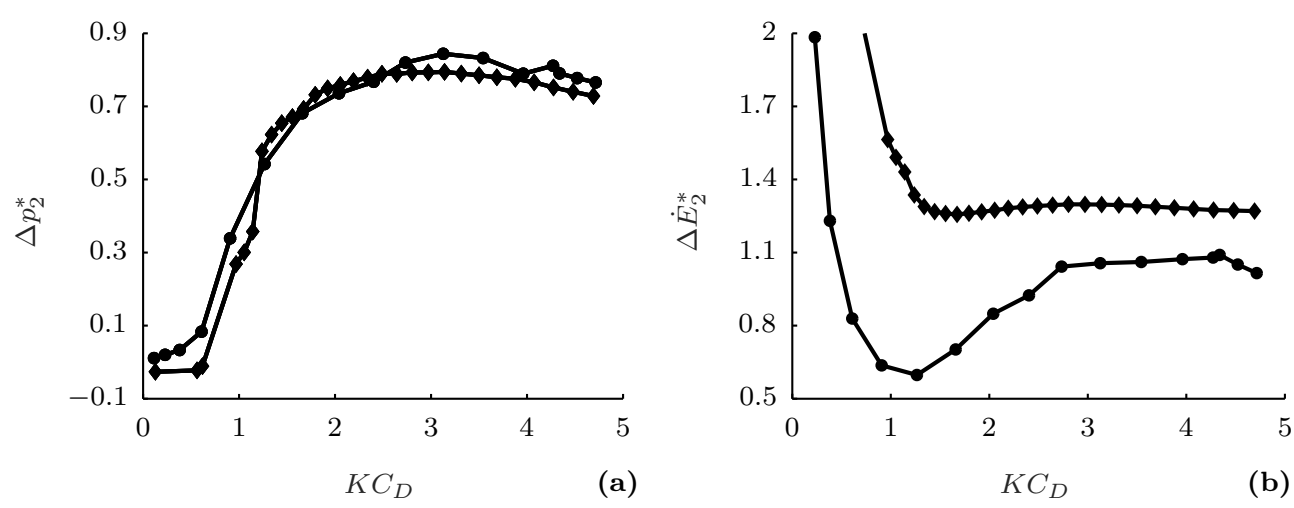

Figure 4.12: (a) Dimensionless pressure drop and (b) acoustic power dissipation as a function of $K C_{D}$ for the numerical simulations (-) and experimental investigation $(\bullet)$ at $80 \mathrm{~Hz}$. Experimental data where the standard deviation of the pressure is larger than $10 \%$ of the mean pressure has been omitted.

the laminar regime, the values for $K C_{D}, \Delta p_{2}^{*}$, and $\Delta \dot{E}_{2}^{*}$ are calculated. A more detailed experimental study on flow separation as well as an extensive description of the experimental setup is presented in Chapter 5 .

Figure 4.12 shows the (a) dimensionless pressure drop and (b) acoustic power dissipation for the experimental and numerical results as a function of $K C_{D}$. For all values of $K C_{D}$, there is a good correspondence between the experimentally and numerically determined dimensionless pressure drop. The initial increase, the maximum, and the following decrease of $\Delta p_{2}^{*}$ are all very similar. This shows that the numerical model is able to accurately predict the dimensionless pressure drop.

The dimensionless acoustic power dissipation (Fig. 4.12b) for the experiments is significantly higher compared with the numerical results for the entire range of $K C_{D}$. However, the trends in $\Delta \dot{E}_{2}^{*}$ are found to be the same. In separate experiments the effect of the surface roughness on the jet pump performance has been investigated (see Appendix D), and it is found that it has a significant effect on $\Delta \dot{E}_{2}^{*}$. The difference is large enough to explain the discrepancy between the experiments with the $3 \mathrm{D}$ printed jet pump and the numerical results, for which surface roughness is not accounted for. This, combined with the good prediction of the dimensionless pressure drop (Fig. 4.12a), establishes enough confidence in the validity of the numerical results presented in this work. Furthermore, the good correspondence shows that the proposed jet pump designs with an increased transition length not only have an improved performance numerically, but also experimentally.

\subsection{Conclusions}

A computational fluid dynamics model is used to investigate the separation of oscillatory flows in jet pumps. A decrease in jet pump performance is shown to be the 
direct result of separation in the diverging flow direction. The time in the period at which flow reversal first occurs reduces for an increasing displacement amplitude. This further degrades the jet pump performance evidenced by a decreasing $\Delta p_{2}^{*}$ as well as an increasing $\Delta \dot{E}_{2}^{*}$.

By increasing the transition length between the small jet pump opening and the tapered surface, the onset of flow separation is shifted to higher displacement amplitudes compared with the reference geometry. This results in an increase of the maximum dimensionless pressure drop from $\Delta p_{2}^{*}=0.66$ for the reference geometry up to $\Delta p_{2}^{*}=0.91$ for an increased transition length of $\Delta L_{J P}=50 \mathrm{~mm}$. Furthermore, the time at which flow reversal first occurs is delayed and the first flow reversal point is shifted to a location with a larger local radius. This reduces the minor losses of the leftward wake formation in the flow separation regime, therewith increasing $\Delta p_{2}^{*}$ and reducing $\Delta \dot{E}_{2}^{*}$. Increasing the transition length of the reference geometry therefore ensures a more robust and effective jet pump design.

The designs with an increased transition length have more acoustic power dissipation in the regime without flow separation due to their elongated geometry. Next to that, the increased length prohibits the design of compact thermoacoustic devices. Therefore, it is shown that these jet pump designs can be made more compact by reducing the radius of the big opening without degrading the jet pump performance. To further increase the compactness, jet pumps with multiple holes or a larger taper angle should be investigated.

The results of the numerical simulations correspond well with the experimental investigation. Nevertheless, all simulations are performed in the laminar regime, since no suitable turbulence model for oscillatory flows exists. To include the effect that turbulence will have on flow separation and the jet pump performance, future work should focus on developing and validating a relatively low-cost turbulence model that can be used in turbulent oscillatory flow. Alternatively, the reduction of flow separation and its effect on the jet pump performance can be investigated experimentally in the turbulent regime. 


\section{Experimental investigation on turbulence and flow separation in jet pumps}

The effect of flow separation and turbulence on the performance of a jet pump in oscillatory flows is investigated. An experimental setup is used to measure the timeaveraged pressure drop as well as the acoustic power dissipation across two different jet pump geometries in a pure oscillatory flow. The results are compared against numerical results where flow separation was found to have a negative effect on the jet pump performance in a laminar flow. Using hot-wire anemometry the onset of flow separation is determined experimentally and the applicability of a critical Reynolds number for oscillatory pipe flows is confirmed for jet pump applications. It is found that turbulence can lead to a reduction of flow separation and hence, to an improvement in jet pump performance compared to laminar oscillatory flows.

\subsection{Introduction}

In the numerical study presented in Chapters $2-3$, four different flow regimes as a function of the jet pump geometry and wave amplitude were determined and it is shown that the applicability of the quasi-steady approximation in laminar oscillatory flows is limited. Due to the taper of the jet pump's through-hole, the flow in the leftward direction can separate from the jet pump wall, resulting in a rightward timeaveraged velocity close to the jet pump wall and a leftward time-averaged velocity at the centerline. The flow separation leads to a significant decrease in the time-averaged pressure drop and to a large deviation from the quasi-steady approximation. The

Adapted from J. P. Oosterhuis, A. A. Verbeek, S. Bühler, D. Wilcox, and T. H. van der Meer. "Flow separation and turbulence in jet pumps for thermoacoustic applications." Flow Turbul. Combust., under review 
onset of flow separation coincides with vortices propagating through the jet pump and is found to be dependent on the Keulegan-Carpenter number, which is based on the diameter of the jet pump waist $\left(D_{s}=2 \cdot R_{s}\right.$ in Fig. 1.5) and the jet pump taper angle $\alpha$ (in radians),

$$
K C_{\alpha}=\frac{\xi_{1}}{D_{s}} \alpha .
$$

For a jet pump geometry with a "smooth" waist $\left(R_{c} / D_{s} \approx 0.36\right)$ and a linear tapered hole, clear flow separation was observed for $K C_{\alpha}>0.7$ and the jet pump performance was significantly reduced. This is in line with the findings of King and Smith on oscillatory flow separation in a two-dimensional diffuser. ${ }^{62}$ They observed that the higher the displacement amplitude, the earlier in the acoustic cycle the flow separates. This results in a larger time-averaged pressure drop in the diverging direction. An increase in the diffuser angle also results in flow separation occurring earlier in the acoustic cycle and in larger minor losses. Furthermore, they found the acoustic Reynolds number to have an impact on the flow separation. Both the time-averaged pressure drop and acoustic power dissipation reduced with increasing Reynolds number, which is an important motivation for the current investigation.

In order to design effective and robust jet pumps, it is important to predict the occurrence of flow separation due to its degrading effect on a jet pump's performance. An important step to achieve this has been presented in Chapter 4 by modifying the wall shape of the jet pump's through-hole. However, all the numerical results presented so far were limited to laminar oscillatory flows in conical jet pumps. In the current chapter the study is extended to the experimental domain to investigate the influence of turbulence and flow separation in conical jet pumps. After a description of the experimental setup in Sec. 5.2, the jet pump performance in terms of the time-averaged pressure drop and acoustic power dissipation is measured (Sec. 5.3). It will be shown that there exists a difference in jet pump performance between the laminar and turbulent regime. Subsequently, hot-wire anemometry is used to further characterize the turbulence and occurrence of flow separation in two different jet pump geometries (Sec. 5.4-5.5).

\subsection{Experimental setup}

The experimental setup is shown schematically in Fig. 5.1 and is, except for the measurement system, the setup previously used by Aben. ${ }^{75}$ On the left side, a loudspeaker (JBL W16GTi) is mounted with a cylindrical back volume; both are structurally decoupled from the rest of the setup by a membrane. A sinusoid signal generated by a computer sound card is amplified using a $2 \mathrm{~kW}$ audio amplifier (Behringer EP2000). The acoustic wave propagates through a horn to the tube section which has an inner diameter of $60 \mathrm{~mm}$ and a total length of $1.2 \mathrm{~m}$. The jet pump is mounted in a $400 \mathrm{~mm}$ long transparent PMMA section of the tube. Vibration of the jet pump samples with respect to the outer tube housing has been ruled out by using high-speed camera visualization at $1000 \mathrm{fps}$ to determine the mutual displacements of the two 


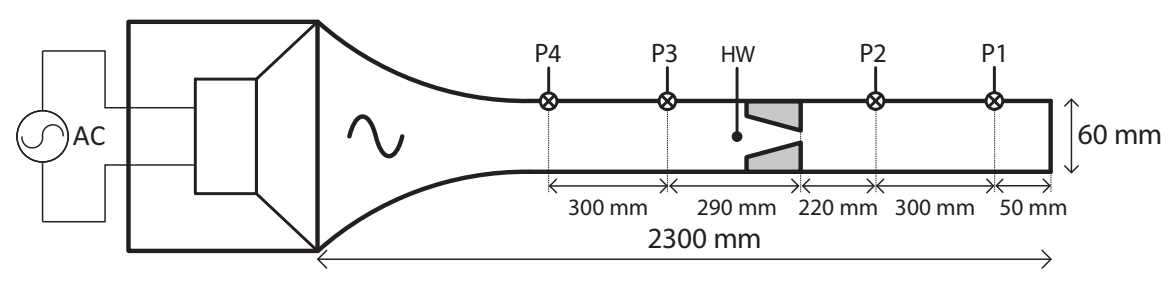

Figure 5.1: Schematic of the experimental setup with the pressure sensors (P1-P4), hot-wire probe (HW) and the jet pump sample. Dimensions not to scale.

parts. The setup is filled with air at ambient conditions. The effect of wave phasing (i.e., standing wave or traveling wave) on the jet pump performance has been investigated previously and no significant differences were observed. ${ }^{91}$ To achieve the maximal acoustic amplitude with the current setup, a closed termination is used in all presented experiments.

\subsubsection{Pressure measurement system}

In order to quantify the jet pump performance, four piezo-resistive differential pressure sensors (Honeywell 26PCAFA6D) are mounted flush with the tube wall. On either side of the jet pump two pressure sensors are located with a mutual distance of $300 \mathrm{~mm}$ (see Fig. 5.1). After amplification, the sensor signals are acquired using a NI-6250 data acquisition device at a sampling frequency of $f_{s}=20 \mathrm{kHz}$ and a sampling time of $T_{s}=1 \mathrm{~s}$. The pressure sensors are dynamically calibrated to a pre-calibrated Kulite XTE-190M pressure sensor in a frequency and pressure amplitude range of $20 \mathrm{~Hz}$ to $150 \mathrm{~Hz}$ and $100 \mathrm{~Pa}$ to $2500 \mathrm{~Pa}$, respectively. The calibration setup consists of a closed tube with a loudspeaker (Monacor SP-60/8) at one end, which is used to generate an acoustic field. At the other side of the tube, the Kulite reference sensor and an uncalibrated Honeywell sensor are mounted flush with the end flange. This dynamic calibration procedure yields a typical averaged sensor sensitivity over the full calibration range of $1 \mathrm{mV} / \mathrm{Pa}$. The standard deviation in the sensitivity is less than $1 \mu \mathrm{V} / \mathrm{Pa}$ across the calibration range. The phase accuracy is determined in the same calibration procedure. A constant time delay is observed yielding a mutual phase difference between the four sensors of less than $0.32^{\circ}$ at $100 \mathrm{~Hz}$. This difference is taken into account as a measurement error. The linearity of the Honeywell pressure sensors is measured using a static water column calibration up to $2500 \mathrm{~Pa}$. The maximum error due to this non-linearity is $\pm 0.10 \%$ of the full-scale range. More details on the pressure measurement system are available in Appendix B.

\subsubsection{Data analysis}

After digitally phase-locking the acquired pressure signals, the pressure amplitude $p_{1}$ is calculated from the discrete Fourier transform at the corresponding driving frequency. The time-averaged pressure $p_{2}$ at each sensor is calculated by averaging 
Table 5.1: Dimensions of jet pump samples. Nomenclature according to Fig. 1.5.

\begin{tabular}{cccccc}
\hline sample & $\alpha$ & $L_{J P}$ & $R_{b}$ & $R_{s}$ & $R_{c}$ \\
\hline 1 & $7^{\circ}$ & $70.5 \mathrm{~mm}$ & $15.0 \mathrm{~mm}$ & $7.0 \mathrm{~mm}$ & $5.0 \mathrm{~mm}$ \\
2 & $15^{\circ}$ & $35.5 \mathrm{~mm}$ & $15.0 \mathrm{~mm}$ & $7.0 \mathrm{~mm}$ & $5.0 \mathrm{~mm}$ \\
\hline
\end{tabular}

the signal over an integer number of wave periods. This removes the contribution of the acoustic wave from the signal. The time-averaged pressure drop over a jet pump sample, $\Delta p_{2}$, is given by the difference in $p_{2}$ from sensors 2 and 3 (see Fig. 5.1).

In order to determine the velocity amplitude in the jet pump waist $u_{1, J P}$ in a noninvasive way, a two-dimensional linear acoustic model of the setup is employed. By relating the calculated velocity amplitude in the jet pump waist to the pressure amplitude at one of the sensor locations, a linear conversion factor between pressure and velocity is determined from the acoustic model. This conversion factor is only dependent on the driving frequency and the position of the jet pump in the setup. A comparison with non-linear, laminar CFD results confirmed that this approach is accurate to within $5 \%$ when the pressure field to the right of the small jet pump opening is used as a reference (i.e., sensor 1 or 2 in Fig. 5.1). This method is further explained in Appendix C.

Furthermore, the measured pressure amplitudes are used to calculate the acoustic power $\dot{E}_{2}$ on either side of the jet pump for which the method of Fusco et al. is used. ${ }^{99}$ By taking the difference between the acoustic power on either side of the jet pump and correcting for dissipative effects in the tube segments, the contribution of the jet pump to the acoustic power dissipation $\Delta \dot{E}_{2}$ is found.

\subsection{Jet pump performance}

Two jet pump samples are investigated, each having a different taper angle. The dimensions are identical to the geometries used in Chapter 2 and are shown in Table 5.1. The samples are manufactured from a Nylon polymer (PA 2200) using a $3 \mathrm{D}$ laser sintering rapid prototyping process and polished. The surface roughness is measured and ranges from $R_{A}=8.5 \mu \mathrm{m}$ to $12 \mu \mathrm{m}$.

Following the quasi-steady approximation (Section 1.3.1), the time-averaged pressure drop across the jet pump is expected to scale with $\left|u_{1, J P}\right|^{2}$ while $\Delta \dot{E}_{2}$ scales with $\left|u_{1, J P}\right|^{3}$. As such, the measured time-averaged pressure drop $\Delta p_{2}$ and acoustic power dissipation $\Delta \dot{E}_{2}$ are normalized according to ${ }^{64}$

$$
\begin{aligned}
\Delta p_{2}^{*} & =\frac{8 \Delta p_{2}}{\rho_{0}\left|u_{1, J P}\right|^{2}}, \\
\Delta \dot{E}_{2}^{*} & =\frac{3 \pi \Delta \dot{E}_{2}}{\rho_{0} \pi R_{s}^{2}\left|u_{1, J P}\right|^{3}},
\end{aligned}
$$




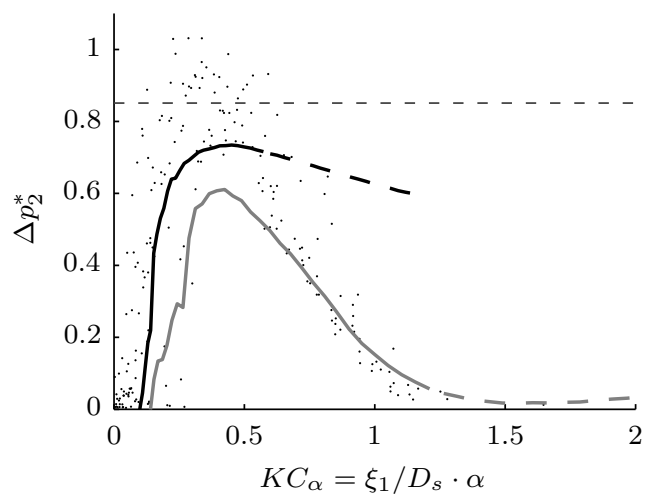

Figure 5.2: Dimensionless pressure drop measured experimentally at $80 \mathrm{~Hz}$ for two jet pump samples: $7^{\circ}$ taper angle jet pump (black line) and $15^{\circ}$ jet pump (gray line). Dots represent numerical results from jet pump geometries with taper angles ranging from $3^{\circ}$ to $20^{\circ}$ and driven at frequencies ranging from $10 \mathrm{~Hz}$ to $200 \mathrm{~Hz}$ reproduced from Chapter 3 . Experimental results where $R e>R e_{c}$ are shown by a dashed line. The horizontal dashed line indicates the predicted performance from the quasi-steady approximation. ${ }^{7}$

with $\Delta p_{2}^{*}$ representing the difference in minor loss coefficients between the backward and forward flow direction and $\Delta \dot{E}_{2}^{*}$ representing the summation of minor loss coefficients, assuming the quasi-steady approximation to be valid. By examining these normalized quantities, any effect flow separation and turbulence have on the jet pump performance becomes more readily visible.

For each jet pump sample, a sweep is executed over the wave amplitude by increasing the audio volume in 50 consecutive steps. At each setpoint, the pressure is recorded for 60 time traces of $1 \mathrm{~s}$ each, and the outcome variables (see Section 5.2) are subsequently averaged. Figure 5.2 shows the dimensionless time-averaged pressure drop as a function of the Keulegan-Carpenter number $K C_{\alpha}$ defined in Eq. 5.1. The lines represent experimental results obtained at $80 \mathrm{~Hz}$ using the $7^{\circ}$ taper angle jet pump (upper black line) and using the $15^{\circ}$ taper angle jet pump (lower gray line). The dots represent numerical results from Chapter 3 using a large variety of jet pump geometries with a taper angle ranging from $3^{\circ}$ to $20^{\circ}$ and simulated at frequencies ranging from $10 \mathrm{~Hz}$ to $200 \mathrm{~Hz}$. All the numerical results show an increase in $\Delta p_{2}^{*}$ at low values of $K C_{\alpha}$. This is a result of minor losses caused by the vortex shedding from the small jet pump opening. As soon as the flow starts to separate from the inside jet pump wall, the dimensionless time-averaged pressure drop stagnates, then drops rapidly when full flow separation without reattachment is observed for $K C_{\alpha}>0.7$. The experimental results (lines in Fig. 5.2) show a similar increase and maximum in $\Delta p_{2}^{*}$. However, the pressure drop tends to stabilize for higher values of $K C_{\alpha}$ and higher Reynolds numbers. Relating this observation to the flow regimes distinguished in the numerical study, the difference in $\Delta p_{2}^{*}$ for $K C_{\alpha}>0.7$ suggests a reduction of flow separation at high Reynolds numbers, especially in the case of the $7^{\circ}$ jet pump sample. A major phenomenon that can explain the hypothesized reduction of flow separation is the occurrence of turbulence. Hence, we define an acoustic Reynolds number based on the viscous penetration depth $\delta_{\nu}=\sqrt{2 \nu / \omega}$ with $\nu$ being the kinematic viscosity, 


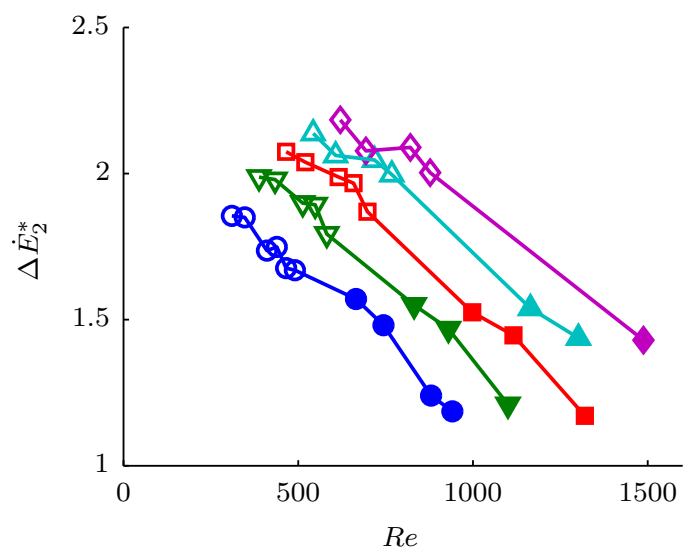

Figure 5.3: Dimensionless acoustic power dissipation measured from two jet pump samples as a function of the acoustic Reynolds number. Each curve represents results at a fixed Keulegan-Carpenter number: $K C_{\alpha}=1.0(\bullet), 1.25(\nabla), 1.5(\square), 1.75(\Delta)$ and $2.0(\bullet)$. The different measurement points are obtained by varying the jet pump geometry (closed symbols for $\alpha=7^{\circ}$, open symbols for $\alpha=15^{\circ}$ ) and driving frequency from $40 \mathrm{~Hz}$ to $100 \mathrm{~Hz}$

$$
R e=\frac{\left|u_{1, J P}\right| \delta_{\nu}}{\nu}
$$

Its critical value for oscillatory pipe flows is defined as ${ }^{83}$

$$
R e_{c}=305\left(\frac{D}{\delta_{\nu}}\right)^{\frac{1}{7}}
$$

In Fig. 5.2, the dashed parts of the curves represent results where $R e>R e_{c}$ and a transition to turbulence can be expected. It is remarkable that in the turbulent regime, little additional decay in $\Delta p_{2}^{*}$ is observed. This suggests a reduction of flow separation and corresponds to the findings of King and Smith on the oscillatory flow in a diffuser. ${ }^{62}$

The effect of flow separation on the jet pump performance is further emphasized by studying the dimensionless acoustic power dissipation (Eq. 5.3). By using two different taper angles and varying the driving frequency, the ratio between $K C_{\alpha}$ and $R e$ is influenced. Hence, the relation between the tendency to flow separation $\left(K C_{\alpha}\right)$ and the momentum of the fluid $(R e)$ can be studied. Figure 5.3 shows the dimensionless acoustic power dissipation as a function of the Reynolds number for various values of $K C_{\alpha}$ in the regime where flow separation can occur. For a given value of $K C_{\alpha}$, the dimensionless acoustic power dissipation decreases with the Reynolds number. Hence, an increase in Reynolds number leads to a reduction of the energy dissipated in the jet pump, which can only be understood if less energy dissipating flow features, such as flow separation, are present. A higher Reynolds number results in the boundary layer better withstanding the adverse pressure gradient that ultimately causes the flow to separate. ${ }^{62}$ The described observations will be further discussed in the next section, supported by velocity measurements close to the jet pump. 


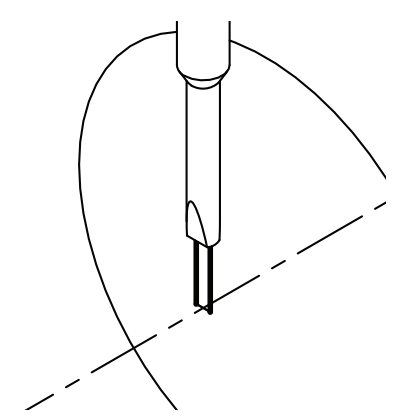

Figure 5.4: Orientation of the Dantec hot-wire probe mounted just outside the big opening of the jet pump (isometric view). The actual hot-wire is situated between the two prongs. The dashed line indicates the jet pump centerline.

\subsection{Flow separation and vortex propagation}

From the numerical investigation in Chapters 2-3 it became clear that the existence of flow separation has a negative effect on a jet pump's performance. Furthermore, in Chapter 4 the onset of flow separation has been shown to coincide with vortices propagating leftward from the jet pump waist through the jet pump during one half of the acoustic period. As such, the occurrence of flow separation can be identified by capturing the leftward propagating vortex. The latter is performed by using hot-wire anemometry to measure the local velocity just outside the jet pump's big opening. A single hot-wire probe is mounted at the centerline, an axial distance of $5 \mathrm{~mm}$ from the jet pump (indicated by "HW" in Fig. 5.1 and shown in detail in Fig. 5.4). The probe is oriented such that the plane spanned by the wire and the wire-prong is perpendicular to the wave propagation direction to minimize the intrusiveness of the hot-wire probe on the flow. A calibration is performed under the same hot-wire orientation using a calibration nozzle in steady flow. ${ }^{100}$ Velocities between $1.8 \mathrm{~m} / \mathrm{s}$ to $40 \mathrm{~m} / \mathrm{s}$ have been calibrated against the pressure drop over the calibration nozzle, which is measured using a water column with a resolution of $1 \mathrm{~Pa}$. This yields an uncertainty in the velocity of less than $5 \%$ for velocities higher than $4 \mathrm{~m} / \mathrm{s}$. The accuracy of the hot-wire measurements is verified by comparing the velocity amplitude with the calculated jet pump waist velocity amplitude (see Section 5.2.2). Assuming incompressible expansion through the jet pump, the velocity amplitude at the hot-wire location is estimated. For operating conditions where no flow separation is expected $\left(K C_{\alpha}<0.7\right)$, the difference is less than $0.5 \mathrm{~m} / \mathrm{s}$. It must be noted that the static calibration method is suitable for velocity amplitude measurements, while errors might occur in measuring velocities around flow reversal. ${ }^{101,102}$ This is considered acceptable for the current purpose of hot-wire measurements. The platinum coated tungsten hotwire has a diameter of $5 \mu \mathrm{m}$, a length of $0.73 \mathrm{~mm}$ and is used in combination with a Dantec 90C10 Constant Temperature Anemometer (CTA) module. ${ }^{100}$ The bandwidth is $75 \mathrm{kHz}$ as was determined using the internal square wave test of the CTA module. The hot-wire signal is captured on a separate system using a NI-9215A BNC data acquisition system at a sampling frequency of $f_{s}=20 \mathrm{kHz}$ and a sampling time of 


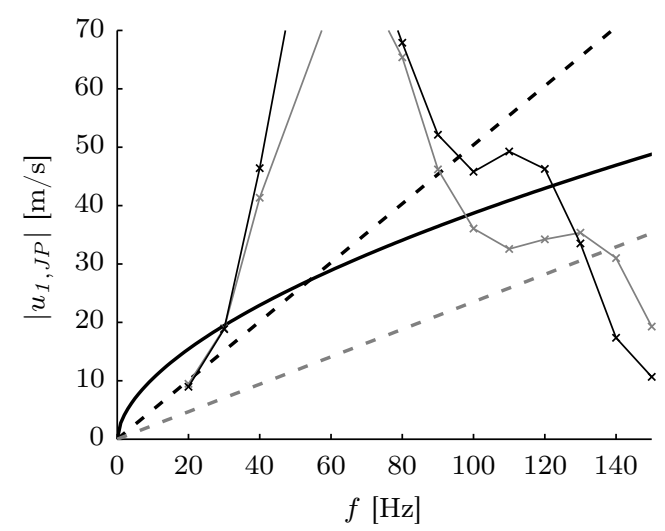

Figure 5.5: Theoretical boundaries of flow regimes as a function of the driving frequency and jet pump velocity amplitude. Black solid line indicates $R e=R e_{c}$, dashed lines indicate $K C_{\alpha}=0.7$ for $\alpha=7^{\circ}$ jet pump (black) and $\alpha=15^{\circ}$ jet pump (gray). Thin lines indicate jet pump velocity amplitude at maximal audio signal amplification attainable with the experimental setup using $\alpha=7^{\circ}$ (black) and $\alpha=15^{\circ}$ (gray) jet pump.

$T_{s}=60 \mathrm{~s}$ for each setpoint. Note that with this single hot-wire configuration no distinction can be made between flow to the left and flow to the right. This means that a pure, harmonic velocity oscillation will lead to a signal shape corresponding to a rectified sine wave and, consequently, to a peak in the frequency spectrum at twice the driving frequency. Any streaming occurring might lead to a shift in the velocity signal, resulting in a signal shape different from the rectified sine wave. The method used to identify the flow separation regardless of the velocity signal shape is described in Section 5.4.2.

Measurements are carried out with the two jet pump samples described in Section 5.3 at three different driving frequencies, $f=40 \mathrm{~Hz}, 80 \mathrm{~Hz}$ and $100 \mathrm{~Hz}$, over the full range of wave amplitudes achievable with the experimental setup. The driving frequency and jet pump geometry have an influence on the velocity amplitude where either flow separation $\left(K C_{\alpha}>0.7\right)$ or turbulence $\left(R e>R e_{c}\right)$ can be expected. Figure 5.5 shows the theoretical boundary between laminar and turbulent flow (thick solid line). The onset of flow separation is shown by the dashed lines. For the $15^{\circ}$ jet pump (lower gray line) the onset of flow separation is expected at lower amplitudes than the transition to turbulence for the investigated frequency range. The two lines cross for the $7^{\circ}$ jet pump at a frequency of $54 \mathrm{~Hz}$. Given the maximum achievable jet pump velocity amplitude with the current experimental setup as a function of the driving frequency (thin lines), the turbulent regime can be reached when driving the setup at a frequency between $40 \mathrm{~Hz}$ to $100 \mathrm{~Hz}$. Hence, this frequency range is chosen for the characterization of the onset of both flow separation and turbulence in the two different jet pump samples. 


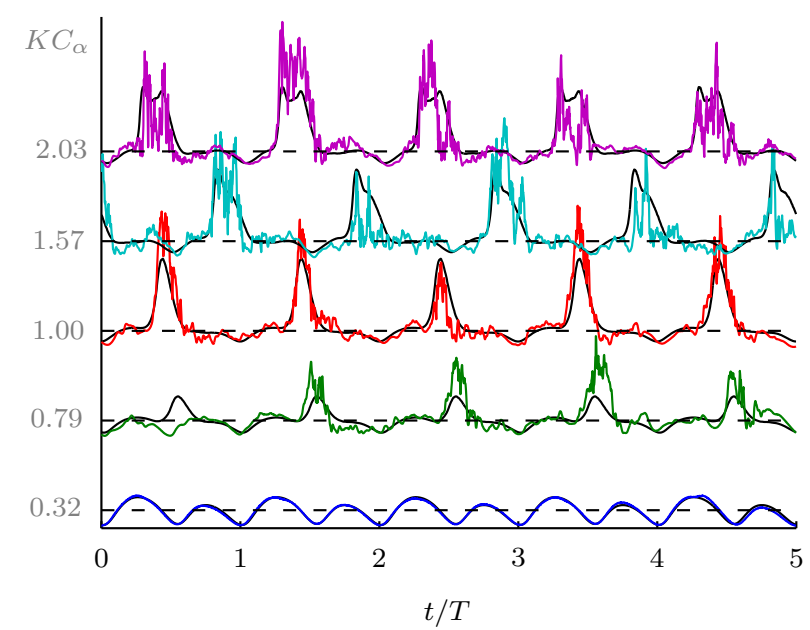

Figure 5.6: Velocity recordings during five consecutive wave periods for $\alpha=15^{\circ}$ jet pump, $f=40 \mathrm{~Hz}$. Traces shown at $K C_{\alpha}=0.32,0.79,1.00,1.57$ and 2.03 corresponding to $R e=98,243$, 311, 487 and 632, respectively. Lines are vertically displaced and normalized by the median of the phase-averaged velocity (Eq. 5.7) to enhance readability. Black solid lines represent phase-averaged velocity, five times repeated in time.

\subsubsection{Velocity time traces}

A sweep over wave amplitude, equal to the jet pump performance measurements carried out in Section 5.3, is executed for each jet pump sample and driving frequency. The velocity signal is recorded for $60 \mathrm{~s}$ per setpoint. This results in 2400 to 6000 wave periods captured per setpoint, depending on the frequency. Figure 5.6 shows the velocity signal for five consecutive periods at various values of $K C_{\alpha}$ for the $15^{\circ}$ taper angle jet pump driven at $40 \mathrm{~Hz}$. From all the recorded wave periods, a phase-averaged velocity is calculated,

$$
\langle u\rangle(\tau)=\frac{1}{N_{p}} \sum_{i=1}^{N_{p}} u(\tau+(i-1) \cdot T),
$$

with $T=1 / f$ the wave period, $\tau$ a relative time ranging from 0 to $T$ and $N_{p}$ the total number of wave periods recorded. The phase-averaged velocity, which is still a function of the relative time $\tau$ or equivalently the wave phasing $\varphi$, is shown by the overlaying black solid lines in Fig. 5.6. The bottom velocity trace at $K C_{\alpha}=0.32$ shows a clean acoustic profile. Hardly any high-frequency perturbations are observed, which is reasonable given that $R e \ll R e_{c}$. The signal has a typical rectified sine shape due to the directional ambiguity of the velocity derived from the hot-wire signal. When the wave amplitude is increased to $K C_{\alpha}=0.79$, a periodic burst in the velocity is observed. This periodic burst is also visible at higher wave amplitudes $\left(K C_{\alpha}=1.0,1.57\right.$ and 2.03 in Fig. 5.6). Furthermore, the amount of high-frequency perturbations increases when the Reynolds number increases. In Section 5.5 these effects will be further quantified. 


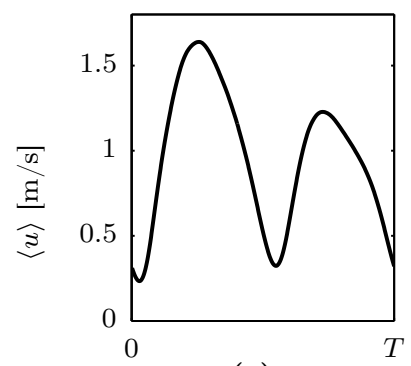

(a)

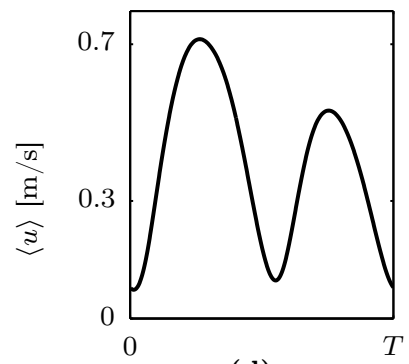

(d)

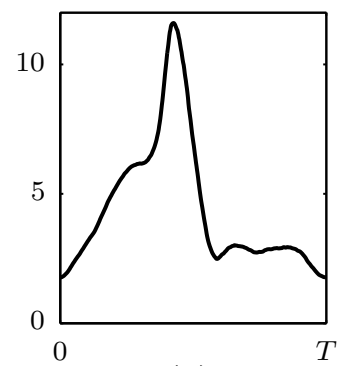

(b)

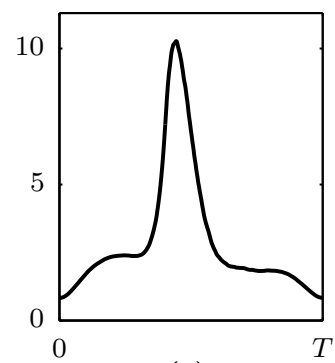

(e)

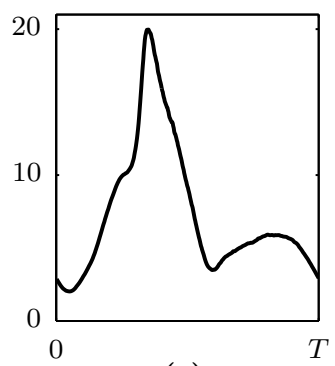

(c)

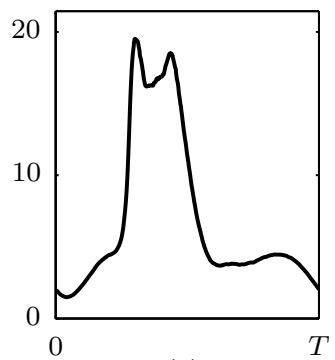

(f)

Figure 5.7: Typical shapes of the phase-averaged velocity $\langle u\rangle$ with $K C_{\alpha}$ increasing from left to right: $(\mathbf{a} \& \mathbf{d})$ pure acoustic profile $K C_{\alpha} \approx 0.3 ;(\mathbf{b} \& \mathbf{e})$ flow separation, single peak, $K C_{\alpha} \approx 1.0$ and (c \& f) flow separation with two sharp peaks, $K C_{\alpha} \approx 2.0$. Results shown for the $\alpha=7^{\circ}$ jet pump (top row, $(\mathbf{a}-\mathbf{c})$ ) and the $\alpha=15^{\circ}$ jet pump (bottom row, $(\mathbf{d}-\mathbf{f})$ ). Frequency is $40 \mathrm{~Hz}$.

\subsubsection{Identification of flow separation and vortex propagation}

To identify the onset of flow separation and the related leftward vortex propagation, the recorded phase-averaged velocity profile is examined. When a vortex passes the hot-wire probe, a periodic burst in the signal is expected due to the temporal high absolute velocity. This is confirmed from CFD simulations where a periodic peak in the velocity signal just outside the jet pump is observed when flow separation occurs.

From the hot-wire measurements, three general shapes of the phase-averaged velocity profile are distinguished and shown in Fig. 5.7 for the $7^{\circ}$ taper angle jet pump (top row) and $15^{\circ}$ taper angle jet pump (bottom row) at $f=40 \mathrm{~Hz}$. At low amplitudes $\left(K C_{\alpha} \ll 0.7\right.$, Fig. $\left.5.7 \mathrm{~d}\right),\langle u\rangle$ has a harmonic shape at twice the driving frequency due to the directional ambiguity of the hot-wire signal. When the amplitude is increased, a separate peak starts to appear in the velocity profiles (Fig. 5.7e) and the velocity profile shows exactly the same features observed in numerical results when leftward vortex shedding and flow separation occurs. At even higher amplitudes $\left(K C_{\alpha}>1.8\right.$, Fig. 5.7f) two closely-spaced sharp peaks are measured. In numerical results this additional peak is also observed and linked to an interaction of the emerging vortex with weak vortex rings that are generated from the edge of the large jet pump opening. For cases with similar $K C_{\alpha}$ but a larger Reynolds number (i.e., at higher frequencies or lower jet pump taper angles than $40 \mathrm{~Hz}$ and $15^{\circ}$ ) the secondary peak is less prominent 


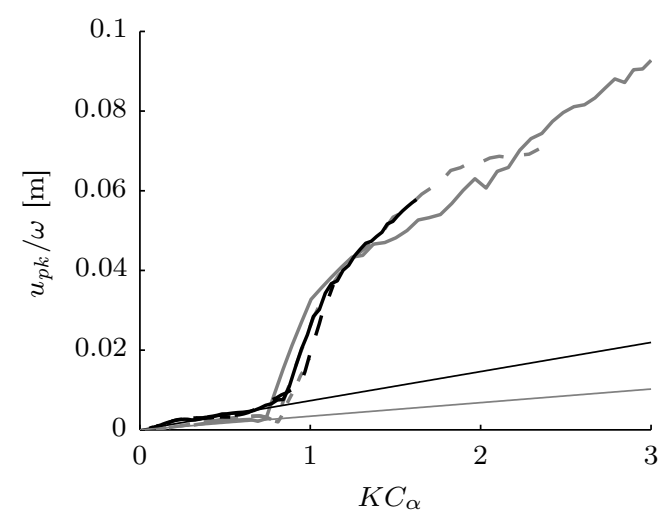

Figure 5.8: Peak height $u_{p k}$ in phase-averaged velocity profile (Eq. 5.8) scaled by the angular frequency $\omega$ and shown as a function of $K C_{\alpha}$. Black lines indicate $\alpha=7^{\circ}$ jet pump, gray lines indicate $\alpha=15^{\circ}$ jet pump. Line styles represent different frequencies: $40 \mathrm{~Hz}$ (solid), $80 \mathrm{~Hz}$ (dashed) and $100 \mathrm{~Hz}$ (dotted). The thin lines show the theoretical course of $u_{p k} / \omega$ in the case of a purely sinusoidal velocity without flow separation.

and probably damped by a higher turbulent intensity. This is visible in Fig. 5.7c for the $\alpha=7^{\circ}$ jet pump.

The height of the periodic peak in the phase-averaged velocity profile caused by leftward vortex propagation, will be used to identify the occurrence of flow separation. To calculate the height of this peak, first an appropriate baseline value from the phase-averaged velocity profile is defined to avoid any mean velocity from affecting the calculated height of the peak. To avoid the flow separation peak itself from influencing this baseline velocity, the median is used instead of the arithmetic mean,

$$
\overline{\langle u\rangle}=\operatorname{median}(\langle u\rangle) \text {. }
$$

Then, the peak height is defined as the distance between the maximum and baseline value of the phase-averaged velocity profile,

$$
u_{p k}=\max \langle u\rangle-\overline{\langle u\rangle}
$$

Figure 5.8 shows the calculated velocity peak as a function of $K C_{\alpha}$ for both jet pump samples and all driving frequencies. By dividing $u_{p k}$ by the (angular) frequency, the contribution of the frequency to the magnitude of the velocity peak is correctly accounted for and all cases collapse to a single curve with $u_{p k} / \omega$ representing an instantaneous displacement amplitude. The thin lines in Fig. 5.8 indicate the theoretical course of $u_{p k} / \omega$ if the flow would be purely oscillatory, i.e. when no flow separation or vortex shedding occurs. This corresponds to a pure sinusoid, assuming the volume flow rate to be equal at the hot-wire location and in the jet pump waist. The theoretical course of $u_{p k} / \omega$ is well approached by the measured values up to the point where the flow separation and leftward vortex propagation is initiated. In all cases, a clear increase in the peak is observed around $K C_{\alpha}=0.7$, which matches well with the onset of flow separation determined in Chapter 3. It becomes clear that 
the Keulegan-Carpenter number is indeed the parameter that determines the onset of flow separation and that the effect of the jet pump taper angle is nicely accounted for in $K C_{\alpha}$.

Using the magnitude of the velocity peak as a measure for the vorticity of the leftward propagating vortex, it can be concluded that the Reynolds number has no effect on the strength of the vortex generated. For a given $K C_{\alpha}$, the Reynolds number differs approximately by a factor of two between the $7^{\circ}$ and $15^{\circ}$ jet pump samples. As the curves in Fig. 5.8 overlay, it becomes clear that there is no effect of the Reynolds number on the height of the velocity peak. Alternatively, the propagation speed of the vortex is quantified by calculating the width of the velocity peak as the time that the phase-averaged velocity $\langle u\rangle$ exceeds its median value $\overline{\langle u\rangle}$ incremented by the standard deviation. For all cases investigated, the peak duration converges to $\Delta t_{p k} / T=0.3$ for $K C_{\alpha}>0.7$. Hence, no significant influence of the Reynolds number is observed, which is also widely reported in literature on vortex rings. ${ }^{4,68,103}$

This behavior might seem to be in contradiction with the measured jet pump performance introduced in Sec. 5.3 where $\Delta p_{2}^{*}$ showed a stabilizing tendency for $R e>R e_{c}$ and the acoustic power dissipation decreased as a function of the Reynolds number for a given value of $K C_{\alpha}$. However, it is important to realize that the peak in the velocity profile is caused by the leftward vortex propagation from the jet pump waist and not directly by the flow separation itself. Although the onset of these two flow phenomena does coincide, it has been discussed that only the flow separation significantly influences the occurring minor losses. ${ }^{62,73} \mathrm{~A}$ detailed investigation of the flow field inside the jet pump is required to directly reveal the behavior of the flow separation as a function of the Reynolds number.

\subsection{Turbulence}

Besides the leftward vortex propagation, the recorded hot-wire signals allow us to analyze the level of turbulence generated by the jet pump. Turbulence in oscillatory pipe flow has been studied extensively. ${ }^{62,83,104}$ In general, turbulence can be characterized by the acoustic Reynolds number (Eq. 5.4) if the tube diameter is sufficiently large $\left(R / \delta_{\nu}>10\right)$. However, the Reynolds number for the oscillatory flow through jet pumps is not uniquely defined because the velocity amplitude is not constant throughout the jet pump. So far we have assumed the jet pump waist, where the velocity amplitude is maximal, to be the point where turbulence is first generated. Depending on the displacement amplitude and the amount of turbulent mixing, the generated turbulent eddies will propagate to the hot-wire measurement location where they will be registered as velocity fluctuations. After calculating the periodic contribution to the velocity signal using the phase-averaged velocity (Eq. 5.6), the fluctuating part of the velocity is calculated from

$$
u^{\prime}(t)=u(t)-\langle u\rangle,
$$




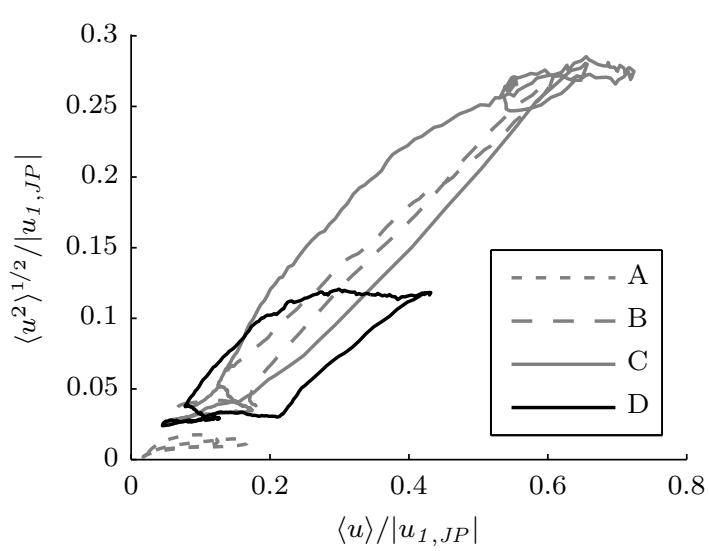

Figure 5.9: Phase-averaged standard deviation $\left\langle u^{2}\right\rangle^{1 / 2}$ plotted against phase-averaged velocity $\langle u\rangle$, both normalized by the velocity amplitude in the jet pump waist $\left|u_{1, J P}\right|$. Four different operating conditions shown as indicated in Table 5.2. In all cases $f=40 \mathrm{~Hz}$.
Table 5.2: Operating conditions corresponding to the different lines shown in Fig. 5.9

\begin{tabular}{cccc}
\hline line & $\alpha$ & $R e / R e_{c}$ & $K C_{\alpha}$ \\
\hline $\mathrm{A}$ & $15^{\circ}$ & 0.19 & 0.32 \\
$\mathrm{~B}$ & $15^{\circ}$ & 0.54 & 0.89 \\
$\mathrm{C}$ & $15^{\circ}$ & 1.86 & 3.10 \\
$\mathrm{D}$ & $7^{\circ}$ & 1.98 & 1.54 \\
\hline
\end{tabular}

and in a similar way as the phase-averaged velocity $\langle u\rangle$, the standard deviation as a function of the relative time $\tau$ is calculated using

$$
\left\langle u^{2}\right\rangle^{1 / 2}(\tau)=\sqrt{\frac{1}{N_{p}} \sum_{i=1}^{N_{p}}\{u(\tau+(i-1) \cdot T)-\langle u\rangle(\tau)\}^{2}} .
$$

There are two phenomena affecting the phase-averaged standard deviation. First, $\left\langle u^{2}\right\rangle^{1 / 2}$ increases as soon as the flow transitions to turbulence due to its random nature. ${ }^{105}$ The second phenomenon resulting in an increased phase-averaged standard deviation is the occurrence of leftward vortex propagation. It was already visible in the velocity traces in Fig. 5.6 that the peaks in the velocity signal, which have been linked to the existence of leftward vortex propagation, do not always occur exactly at the same phase and vary in strength from period to period. This also results in a strong increase in the phase-averaged standard deviation. Consequently, the phaseaveraged standard deviation by itself is not sufficient to uniquely identify the onset of turbulence in the current situation.

Nevertheless, there is one major difference in how the leftward vortex propagation and turbulence influence the phase-averaged standard deviation. This is illustrated in Fig. 5.9 by plotting the phase-averaged standard deviation against the phase-averaged velocity, both normalized using the jet pump waist velocity amplitude, and shown for various operating conditions. When only vortex propagation occurs (dashed gray line, $R e / R e_{c}=0.54$ and $K C_{\alpha}=0.89$ ), the peak in the phase-averaged velocity occurs simultaneously with an increased phase-averaged standard deviation. This leads to a very narrow loop where the increase and decrease in both quantities follow almost the same line. When the critical Reynolds number is exceeded (black solid line, $\left.R e / R e_{c}=1.98, K C_{\alpha}=1.54\right)$, the loop has a totally different shape. Following 

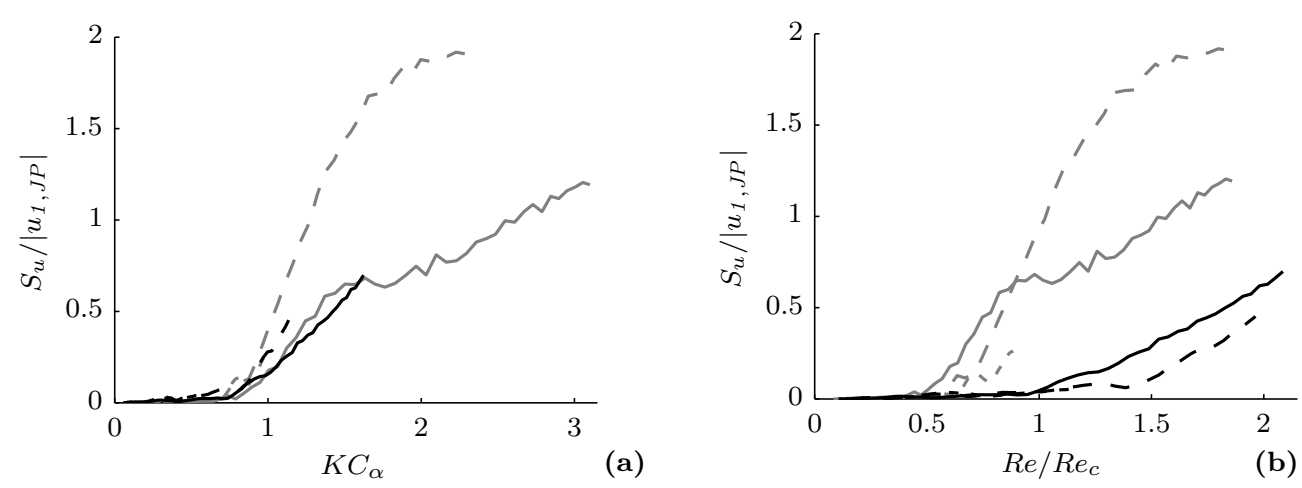

Figure 5.10: Area $S_{u}$ enclosed by $\left(\langle u\rangle,\left\langle u^{2}\right\rangle^{1 / 2}\right)$-loops (see Fig. 5.9), scaled with the jet pump waist velocity $\left|u_{1, J P}\right|$ and plotted against (a) $K C_{\alpha}$ and (b) $R e / R e_{c}$. Black lines indicate results using the $\alpha=7^{\circ}$ jet pump, gray lines indicate $\alpha=15^{\circ}$ jet pump. Line styles represent different frequencies: $40 \mathrm{~Hz}$ (solid), $80 \mathrm{~Hz}$ (dashed) and $100 \mathrm{~Hz}$ (dotted).

the black line in a counter-clockwise direction, the standard deviation initially stays low until a certain transition velocity is reached, then $\left\langle u^{2}\right\rangle^{1 / 2}$ rapidly increases until $\langle u\rangle$ reaches a maximum. When the phase-averaged velocity subsequently decreases, the standard deviation does not decrease immediately but lags with respect to the phase-averaged velocity. This is caused by the relaminarization of the flow which takes more time than the earlier transition to turbulence and occurs every period. ${ }^{106-108}$ The wide hysteresis loops are observed for all cases where $R e>R e_{c}$. The solid gray line in Fig. 5.9 represents a situation where both strong vortex propagation and turbulence occur for the $\alpha=15^{\circ}$ jet pump. This results in a wider shape compared to the laminar case (dashed gray line). For the situation where both $R e$ and $K C_{\alpha}$ are below their critical values (gray dotted line), no significant standard deviation is measured and due to the absence of flow separation the phase-averaged velocity stays low, even when normalized by the jet pump waist velocity amplitude. The cases shown are exemplary for all measured operating conditions, taking into account the flow regime boundaries defined by $R e$ and $K C_{\alpha}$.

To further quantify the effect that both vortex propagation and turbulence have on the phase-averaged standard deviation, the area enclosed by the loops in Fig. 5.9 is calculated. Figure 5.10 shows the enclosed area, $S_{u}$, for both jet pump samples (black and gray lines) and all frequencies (dotted, dashed and solid lines) as a function of $K C_{\alpha}$ (Fig. 5.10a) and $R e / R e_{c}$ (Fig. 5.10b). As soon as leftward vortex propagation and flow separation occur (from $K C_{\alpha}=0.7$ ), resulting in narrow-shaped loops in Fig. 5.9, a coherent increase in the enclosed area is observed (Fig. 5.10a). The effect of the turbulence on $S_{u}$ becomes visible in Fig. 5.10b. For $R e / R e_{c}>1$, the enclosed area eventually increases with roughly the same slope for all cases. This suggests that the enclosed area is proportional to the increase in Reynolds number due to the aforementioned hysteresis in $\left\langle u^{2}\right\rangle^{1 / 2}$. The fact that the different curves do not overlay one another and that the $15^{\circ}$ jet pump at $40 \mathrm{~Hz}$ (dashed gray line) has not reached a linear increase as a function of $R e / R e_{c}$ may be caused by the difference in 


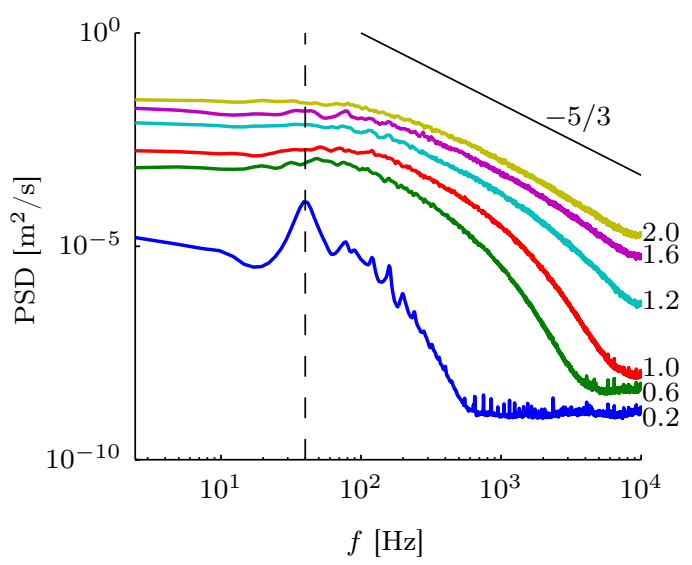

Figure 5.11: Power spectral density at various acoustic Reynolds numbers: $R e / R e_{c}=0.2,0.6$, $1.0,1.2,1.6$ and 2.0 (right axis), using the $\alpha=7^{\circ}$ jet pump driven at $f=40 \mathrm{~Hz}$ as indicated by vertical dashed line. Slope of Kolmogorov turbulent spectrum is illustrated by black solid line.

$K C_{\alpha}$ among the various cases and thus, a different influence of the leftward vortex propagation.

The applicability of the critical Reynolds number (Eq. 5.5) as a predictor for turbulence in the oscillatory flow in jet pumps is emphasized by investigating the frequency spectra. Figure 5.11 shows the power spectral density (PSD) using the $7^{\circ}$ jet pump driven at $40 \mathrm{~Hz}$. The PSD is calculated from the fluctuating part of the velocity signal $u^{\prime}$, which results in a frequency spectrum where the driving frequency and all its higher harmonics are not included. The method of Welch is used to calculate the PSD dividing the velocity signal in blocks of ten wave periods with $50 \%$ overlap each. ${ }^{109}$ After applying a Hamming window, the PSD is calculated by Fourier transforming the signal. The individual lines in Fig. 5.11 each represent a different Reynolds number, shown as a ratio to the critical Reynolds number. The spectra at low Reynolds numbers $\left(R e / R e_{c}<1\right)$ decay more rapidly at higher frequencies than the spectra at higher Reynolds numbers. As the Reynolds number exceeds its critical value, the spectra start following a $-5 / 3$ power law decay. Although the flow at the hot-wire location is far from uniform, the energy spectrum does correspond to a theoretical Kolmogorov spectrum for homogeneous isotropic turbulence. ${ }^{105}$ For the other cases investigated, the frequency spectra show a similar behavior as a function of $R e / R e_{c}$. This analysis underlines the applicability of a critical Reynolds number for oscillatory pipe flows (Eq. 5.5) to jet pumps.

\subsection{Conclusions}

The performance of two jet pump samples is determined experimentally in terms of the time-averaged pressure drop and the acoustic power dissipation. The results are compared with numerical results from Chapter 3. Good correspondence is found 
between numerically predicted jet pump performance and experimental results for Reynolds numbers in the laminar regime. However, in the experimental results the dimensionless time-averaged pressure drop stabilizes for Reynolds numbers larger than the critical Reynolds number. Furthermore, for a given Keulegan-Carpenter number $K C_{\alpha}$, the dimensionless acoustic power dissipation decreases as a function of the Reynolds number. Both these findings indicate that the negative effect flow separation has on the jet pump performance is reduced in the turbulent regime.

Hot-wire anemometry near the jet pump big opening is used to study the onset of flow separation and turbulence in the jet pump. The occurrence of vortex propagation through the jet pump and the related flow separation is identified from periodic peaks in the recorded velocity signal. The onset of flow separation is observed for $K C_{\alpha}>0.7$ for all jet pump samples and frequencies investigated. This is fully in line with numerical results.

Furthermore, we have shown that the Reynolds number calculated at the jet pump waist is a correct predictor for the occurrence of turbulence in the oscillatory flow in jet pumps. For $R e>R e_{c}$ the power spectral density follows the classical $-5 / 3$ Kolmogorov spectrum. Additionally, for $R e>R e_{c}$ a hysteresis in the phase-averaged standard deviation was found which is attributed to the periodic relaminarization of the flow taking more time than the transition to turbulence.

Although the measured jet pump performance together with the defined onset of flow separation and the transition to turbulence all strongly support the hypothesis that the flow separation is reduced at high Reynolds numbers, further research is required to decisively conclude this. Supported by literature on flow separation in steady flows, the pressure gradient along the jet pump wall is of interest to determine both the location and duration of the flow separation. Moreover, the effect of the wall roughness on both the flow separation as well as on the generation of turbulence is subject to future research.

A better understanding of the flow separation inside jet pumps is shown to be key in understanding and predicting the performance of jet pumps. Design adjustments that reduce the flow separation in jet pumps with high taper angles could improve the jet pump effectiveness while maintaining a compact design. 


\section{On the performance and flow characteristics of jet pumps with multiple orifices}

The design of compact thermoacoustic devices requires compact jet pump geometries, which can be realized by employing jet pumps with multiple orifices. In this chapter, the performance of jet pumps having 1 to 16 orifices is characterized experimentally in terms of the time-averaged pressure drop and acoustic power dissipation. Upon increasing the number of orifices, a significant decay in the jet pump performance is observed. Further analysis shows a relation between this performance decay and the diameter of the individual holes. Possible causes of this phenomenon are discussed. Flow visualization is used to study the differences in vortex ring interaction from adjacent jet pump orifices. The mutual orifice spacing is varied and the corresponding jet pump performance is measured. The orifice spacing is shown to have less effect on the jet pump performance compared to increasing the number of orifices.

\subsection{Introduction}

Traditional jet pump designs consist of one or several orifices that have a gentle taper angle to avoid possible flow separation. These gentle taper angles combined with the required difference in cross-sectional areas to obtain the desired time-averaged pressure drop, result in a relatively large component which then has to be integrated inside the thermoacoustic device. In order to manufacture compact thermoacoustic devices, a more compact jet pump design is desirable. Reducing the length of a jet pump can be achieved by moving the two openings closer to one another, increasing

Adapted from J. P. Oosterhuis, M. A. G. Timmer, S. Bühler, D. Wilcox, and T. H. van der Meer. "On the performance and flow characteristics of jet pumps with multiple orifices." J. Acoust. Soc. Am., under review 
its taper angle. However, in the preceding chapters it is shown that this can easily result in the flow separating from the jet pump inner wall. Flow separation inside the jet pump has a devastating effect on the jet pump performance and should be avoided.

Alternatively, the size of a jet pump can be reduced by employing multiple parallel orifices. This reduces the jet pump length while the total cross-sectional areas and the taper angle remain unchanged. One record of a jet pump with a large number of holes is the work of Haberbusch et al., employing a total of 109 holes. ${ }^{34,55}$ Wilcox and Spoor have proposed a similar approach, which is carried out in light of the current research project. ${ }^{56} \mathrm{~A}$ jet pump with 16 holes was used with a total length of less than $20 \mathrm{~mm}$. However, so far the effect this design approach has on a jet pump's performance is neither included in the current design methodology, nor investigated experimentally. The possible interaction of the flow through adjacent orifices has been discussed in literature on synthetic jet arrays. Watson et al. distinguished three different flow regimes based on orifice spacing in synthetic jet arrays: distinct vortex rings without interaction for well-spaced orifices, distinct formation of vortex rings that merge further downstream for an intermediate orifice spacing, and single vortex ring formation for orifices that are spaced very close together. ${ }^{111}$ Similar vortex ring merging has been observed by Smith and Glezer for rectangular synthetic jets spaced apart at 3.3 times the orifice width. ${ }^{112}$ However, the work on synthetic jet actuators focuses on maximizing the total vorticity generated and the consequence of the orifice spacing on the minor losses is, to the authors knowledge, not discussed so far.

In this chapter, the performance of various jet pump geometries having two different taper angles and five different orifice configurations is investigated experimentally. The effects that can be attributed to either a reduction in orifice diameter or to the interaction of the mutual orifices are investigated. Flow visualization is employed to qualitatively determine this interaction. After a brief description of the experimental setup in Section 6.2, the time-averaged pressure drop and acoustic power dissipation of the various jet pump samples are presented in Section 6.3.1. A separate analysis on the effect of the orifice spacing is discussed in Section 6.3.2. These results are further interpreted by the flow visualization discussed in Section 6.4.

\subsection{Experimental setup}

Two different configurations of the experimental setup are used: one for the jet pump performance measurements, and one for the flow visualization experiments (see Section 6.4). The experimental setup used for the jet pump performance measurements is identical to the setup described in Chapter 5 and illustrated in Fig. 6.1. An extensive description of the experimental methods can be found in Section 5.2 on page 68. Details on the pressure sensor calibration are available in Appendix B. Each data point presented in the results that follow is an average of 60 separately acquired samples of $1 \mathrm{~s}$ each. The standard deviation across these 60 samples is considered a measurement uncertainty and the propagation of error approach is used to calculate the resulting error on the derived quantities used in this chapter. ${ }^{113}$ Data points where the stan- 


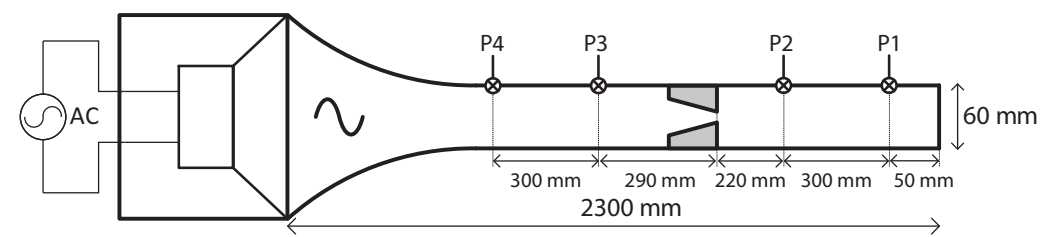

Figure 6.1: Schematic of experimental setup with pressure sensors (P1-P4) and single hole jet pump sample (marked gray). Not to scale.

dard deviation on the normalized pressure drop $\Delta p_{2}^{*}$ is larger than 0.02 are excluded from further analysis.

A direct, but non-intrusive measurement of the velocity amplitude inside the jet pump's small opening (jet pump "waist") is not feasible with the current experimental setup. Instead, the same acoustic model is used as was introduced in Section 5.2.2 and is described in Appendix C. Furthermore, conservation of mass dictates that an equal total cross-sectional area yields an equal velocity amplitude in each jet pump hole. This was confirmed experimentally for synthetic jets. ${ }^{111}$ Consequently, the linear conversion factor does not change for jet pump samples No. 1 to 8 (see Table 6.1) and sensor P2 in Fig. 6.1 is used as the reference sensor for the calculation of the velocity amplitude.

\subsection{Jet pump performance}

The jet pump performance in terms of the dimensionless pressure drop and acoustic power dissipation will be discussed for the different samples that have been investigated. To cover a wide range of $K C_{D}$, measurements are carried out at $40 \mathrm{~Hz}$ and $100 \mathrm{~Hz}$. At each driving frequency, the wave amplitude is swept over the full range attainable by the audio amplifier used.

\subsubsection{Number of orifices}

Eight samples have been investigated with two different taper angles $\left(7^{\circ}\right.$ and $\left.15^{\circ}\right)$ and five different orifice arrangements. The total cross-sectional area of both the small and big openings for each jet pump is kept constant throughout the geometry variations. Furthermore, the ratio of the jet pump curvature $R_{c}$ to the jet pump waist diameter $D_{s}$ is constant. This results in an equal predicted performance based on the quasisteady approximation. ${ }^{7}$ An overview of the relevant dimensions is shown in Table 6.1. The orifices in the jet pumps with 4,8 and 16 holes are oriented in a circular manner as shown in Fig. 6.2. The circular hole pattern diameter is adjusted to fit all orifices within the available outer $60 \mathrm{~mm}$ tube diameter. This results in a $\varnothing 30 \mathrm{~mm}$ hole pattern diameter for the jet pump having 4 holes, a $\varnothing 35 \mathrm{~mm}$ hole pattern diameter for the jet pump having 8 holes and two staggered hole pattern diameters of $\varnothing 20 \mathrm{~mm}$ and $\varnothing 40 \mathrm{~mm}$ for the jet pump with 16 holes. The samples are manufactured from a 
Table 6.1: Jet pump samples with varied number of holes (dimensions in mm unless otherwise stated). Nomenclature corresponds to Fig. 1.5 and $d_{h}$ is the minimal center-to-center distance between adjacent holes.

\begin{tabular}{rcccccccc}
\hline No. & symbol & $\alpha$ & \# orifices & $L_{J P}$ & $R_{s}$ & $R_{b}$ & $R_{c}$ & $d_{h}$ \\
\hline 1 & $\bullet$ & $7^{\circ}$ & 1 & 70.5 & 7.0 & 15.0 & 5.0 & - \\
2 & $\mathbf{\square}$ & $7^{\circ}$ & 2 & 49.8 & 4.9 & 10.6 & 3.5 & 30.0 \\
3 & $\boldsymbol{\nabla}$ & $7^{\circ}$ & 4 & 35.2 & 3.5 & 7.5 & 2.5 & 21.2 \\
4 & $\bullet$ & $7^{\circ}$ & 8 & 24.9 & 2.5 & 5.3 & 1.8 & 13.4 \\
5 & $\star$ & $7^{\circ}$ & 16 & 17.6 & 1.8 & 3.8 & 1.3 & 10.0 \\
6 & $\bigcirc$ & $15^{\circ}$ & 1 & 35.5 & 7.0 & 15.0 & 5.0 & - \\
7 & $\square$ & $15^{\circ}$ & 2 & 25.1 & 4.9 & 10.6 & 3.5 & 30.0 \\
8 & \multirow{2}{*}{} & $15^{\circ}$ & 16 & 8.8 & 1.8 & 3.8 & 1.3 & 10.0 \\
\hline
\end{tabular}

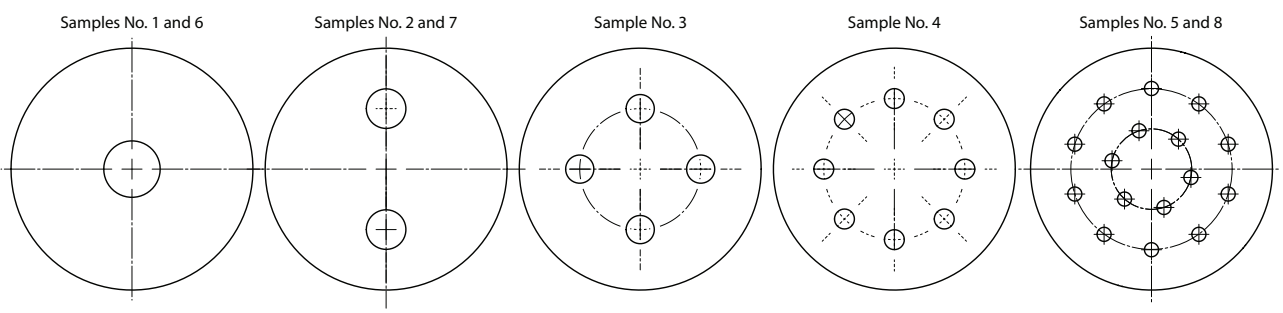

Figure 6.2: Orifice arrangement in jet pump samples No. 1 to 8 . View from small opening side of the jet pump. Dimensions of the individual orifice geometry are specified in Table 6.1.

Nylon polymer (PA 2200) using a 3D laser sintering rapid prototyping process and subsequently polished. This resulted in a typical surface roughness of $R_{A}=8.5 \mu \mathrm{m}$ to $12.0 \mu \mathrm{m}$.

Fig. 6.3a shows the dimensionless pressure drop achieved for the different jet pump samples as a function of $K C_{D}$. Each sample is depicted by a symbol as indicated in Table 6.1. The open symbols correspond to jet pumps with a $15^{\circ}$ taper angle and the closed symbols correspond to jet pumps having a $7^{\circ}$ taper angle. It has been shown that for laminar oscillatory flows the dimensionless pressure drop ultimately decreases to zero due to the occurrence of flow separation. ${ }^{85}$ However, we observe that all curves tend to converge to a constant $\Delta p_{2}^{*}$ for high values of $K C_{D}$. This has been attributed to the onset of turbulence, ${ }^{98}$ which is supported by the graph in Fig. $6.3 \mathrm{~b}$ where the stabilizing trend is only visible for $R e / R e_{c}>1$. A similar relation between flow separation and turbulence has been reported by King and Smith for the oscillating flow in a diffuser. ${ }^{62}$ The stabilizing effect of turbulent flow on the dimensionless pressure drop results in a clear difference in performance between the $7^{\circ}$ and $15^{\circ}$ jet pump samples, mainly due to a difference in the onset of flow separation which is discussed in more detail in the following.

The fact that the jet pump performance stabilizes for $R e>R e_{c}$ allows us to average the jet pump dimensionless pressure drop and dimensionless acoustic power dissipation for $R e>R e_{c}$ without losing significant details. Figure 6.4 shows $\Delta p_{2}^{*}$ averaged 


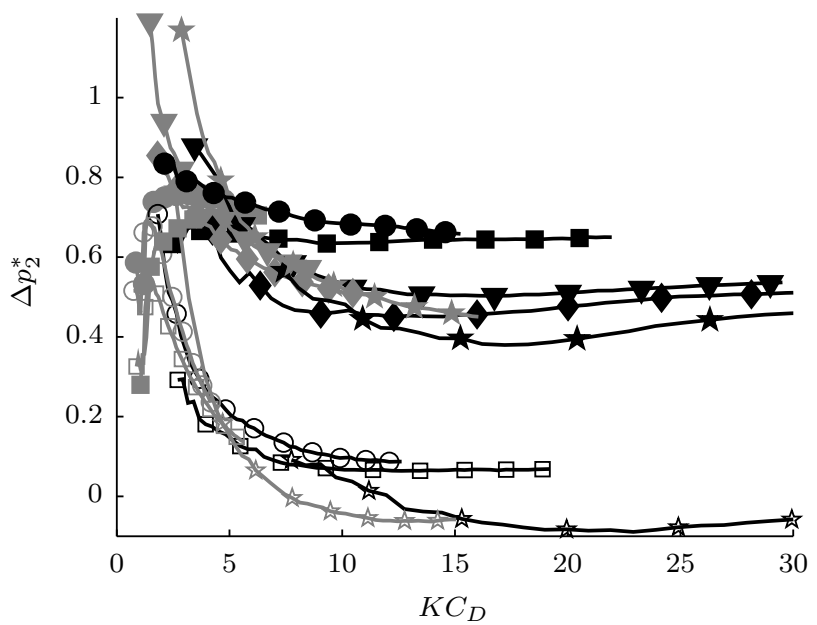

(a)

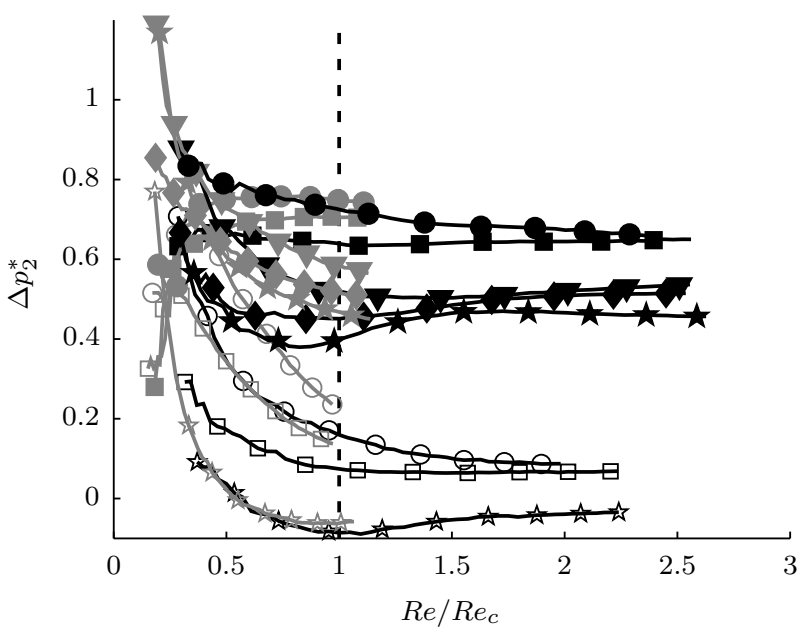

(b)

Figure 6.3: Dimensionless pressure drop as a function of $K C_{D}$ (a) and $R e / R e_{c}$ (b). Different marker types are used to indicate the number of holes as depicted in Table 6.1. For clarity, markers are shown for one fifth of the measured points. Gray corresponds to measurements at $f=100 \mathrm{~Hz}$, black to $f=40 \mathrm{~Hz}$. 


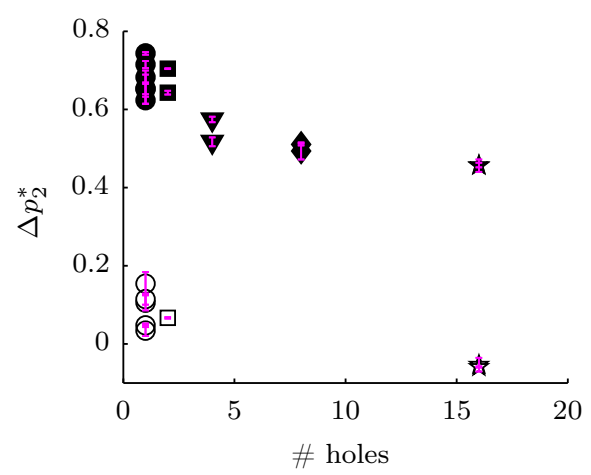

Figure 6.4: Dimensionless time-averaged pressure drop, averaged for $R e>R e_{c}$ and shown as a function of the number of holes in the jet pump sample. The marker types correspond to different jet pump samples (Table 6.1). Error bars indicate the standard deviation of averaging.

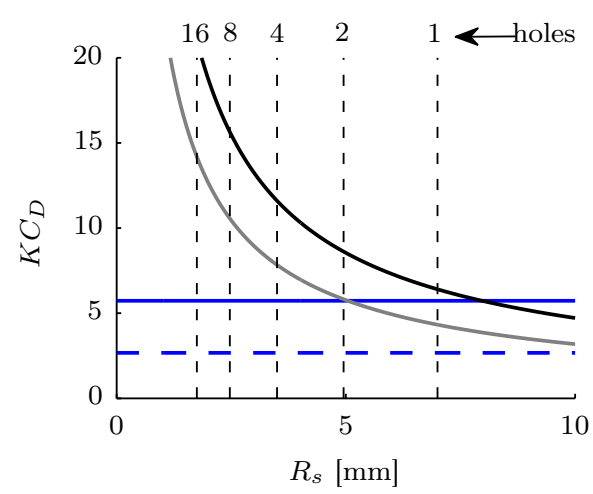

Figure 6.5: Theoretical onsets of flow separation and turbulence in the $\left(R_{s}, K C_{D}\right)$-plane. Curves represent $R e=R e_{c}$ for $f=40 \mathrm{~Hz}$ (black line) and $f=100 \mathrm{~Hz}$ (gray line). Blue lines indicate onset of flow separation at $K C_{\alpha}=0.7$ for a $7^{\circ}$ (solid line) and $15^{\circ}$ (dashed line) taper angle. Vertical thin dotted lines are placed at jet pump waist radii corresponding to the samples used in this study, the number of holes are indicated at the top.

over the full measurement range where $R e>R e_{c}$. The error bars represent the standard deviation across the averaging range. For the single hole jet pumps (samples 1 and 6), additional measurements have been performed at frequencies of $30 \mathrm{~Hz}, 50 \mathrm{~Hz}$, $80 \mathrm{~Hz}$ and $90 \mathrm{~Hz}$. From Fig. 6.4 it becomes clear that the jet pump taper angle does not uniquely determine a jet pump's performance. In other words, the effect of both the number of orifices as well as the frequency cannot be neglected. A clear decay in the dimensionless time-averaged pressure drop is observed for an increase in the number of orifices. In the following, we will examine possible physical explanations of this observation.

It is important to realize the implications of a change in the number of orifices on other parameters that can influence the jet pump's performance. Although the total cross-sectional area has not changed, increasing the number of orifices does inherently lead to a decrease in the diameter of the individual holes. Together with the frequency, this changes the velocity amplitude at which flow separation is first expected (determined by $K C_{\alpha}=0.7$ for the current jet pump curvature) as well as the onset of turbulence (determined by $R e_{c}$ ). ${ }^{85,98}$ This is illustrated in Fig. 6.5 showing the theoretical transitional $K C_{D}$ values for both the onset of turbulence (black and gray lines) and the onset of flow separation (blue lines) as a function of the size of the jet pump waist. Small jet pump holes, which in this study is associated with a large number of holes, leads to the onset of turbulence being shifted to large values of $K C_{D}$ while the onset of flow separation remains unaffected in terms of $K C_{D}$. The latter is independent of frequency or orifice size and depends only on the jet pump taper angle. 

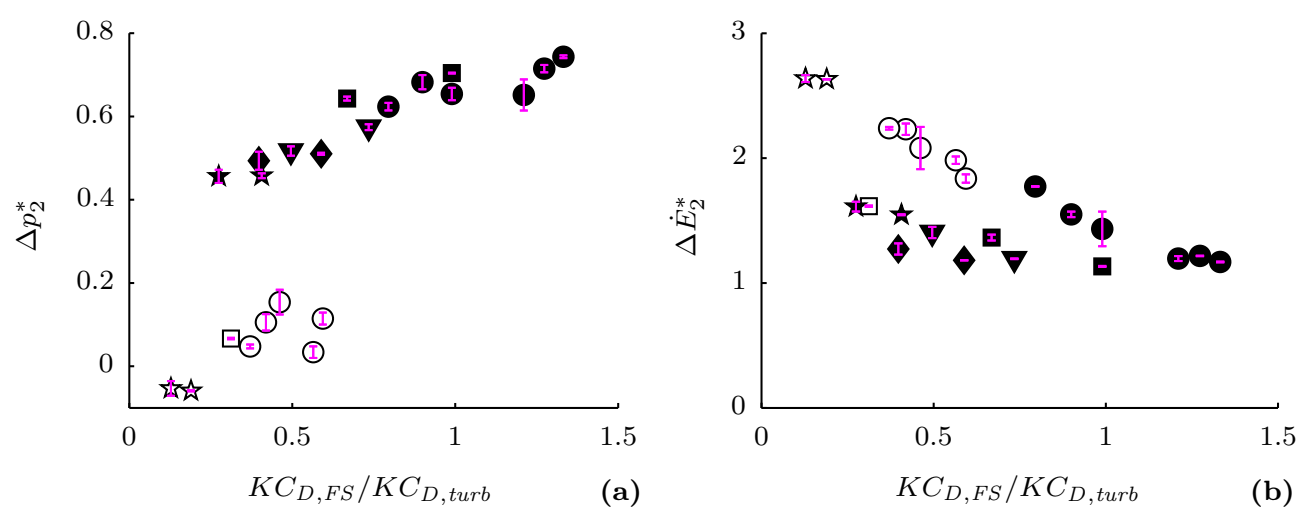

Figure 6.6: Averaged dimensionless pressure drop (a) and acoustic power dissipation (b) as a function of the $K C_{D}$ ratio defined in Eq. 6.1. The marker types correspond to different jet pump samples (Table 6.1). Error bars indicate the standard deviation of averaging.

Consequently, the onset of turbulence and flow separation can be related to each other using a ratio of the form

$$
K C_{D, F S} / K C_{D, t u r b}=\frac{K C_{D}\left(K C_{\alpha}=0.7\right)}{K C_{D}\left(R e=R e_{c}\right)} .
$$

Plotting the results from Fig. 6.4 as a function of this $K C_{D}$ ratio, yields the plot shown in Fig. 6.6a. The solid markers represent results using $7^{\circ}$ jet pump samples where an increasing trend in the turbulent averaged $\Delta p_{2}^{*}$ is visible. This shows that the larger the amplitude at which flow separation first occurs compared to the amplitude where turbulence begins to play a role, the higher the turbulent averaged dimensionless pressure drop becomes. For the $15^{\circ}$ jet pump samples this trend is less apparent. Nevertheless, the fact that the overall $\Delta p_{2}^{*}$ is lower is a direct consequence of flow separation in the diverging flow direction. ${ }^{85}$ Fig. $6.6 \mathrm{~b}$ shows the averaged dimensionless acoustic power dissipation plotted against the $K C_{D}$ ratio. In general, a slight decreasing trend is observed for an increasing $K C_{D}$ ratio. Furthermore, the mutual differences caused by a change in the number of holes (different symbols) are smaller than the differences caused by a change in the taper angle or frequency (equal symbols).

In addition to the influences of turbulence and flow separation on the jet pump performance, the velocity profile can change when the jet pump diameter becomes small compared to the viscous penetration depth $\delta_{\nu}$. This can affect the resulting minor loss coefficients as was analyzed by Wakeland and Keolian for cross-sectional area changes in oscillatory flows. ${ }^{59}$ As such, the parameter $R_{s} / \delta_{\nu}$ should also be taken into consideration. However, both $R_{s} / \delta_{\nu}$ and Eq. 6.1 are linearly dependent on the jet pump waist diameter which results in similar trends. Although the frequency dependency of both scaling parameters is different $\left(R_{s} / \delta_{\nu} \propto f^{1 / 2}\right.$, Eq. $\left.6.1 \propto f^{10 / 7}\right)$, the frequency range investigated is insufficient to draw conclusions about the individual contributions to the jet pump performance. 
Table 6.2: Jet pump samples with varied orifice spacing. Samples contain two holes with a size equal to jet pump sample No. $3\left(D_{s}=7.0 \mathrm{~mm}, \alpha=7^{\circ}\right.$, see Table 6.1$) . d_{h}$ is the center-to-center distance between adjacent holes.

\begin{tabular}{rcl}
\hline No. & $d_{h} / D_{s}$ & $d_{h}[\mathrm{~mm}]$ \\
\hline 9 & 2.7 & 18.9 \\
10 & 3.0 & 21.0 \\
11 & 3.5 & 24.5 \\
12 & 4.0 & 28.0 \\
13 & 5.0 & 35.0 \\
\hline
\end{tabular}

\subsubsection{Orifice spacing}

A complicating factor, is the possible interaction of the flow through adjacent jet pump orifices and the effect this has on the overall performance. For the design and application of synthetic jets a limiting spacing of $d_{h}>4 D_{\mathrm{s}}$ is found adequate to avoid interactions between adjacent vortex rings in order to maximize the overall circulation. ${ }^{111}$ For smaller $d_{h}$, the separate vortex rings start to merge and the overall circulation is reduced. However, the extent to which the vortex ring interaction influences the magnitude of the minor losses is not discussed. For the aforementioned jet pump samples, the spacing ranges from $2.7 D_{\mathrm{s}}$ to $3.1 D_{\mathrm{s}}$, which is just in the range for which vortex interaction can be expected. ${ }^{111}$ As the space available in the setup does not allow the placement of individual orifices further apart, five additional samples are manufactured to study the effect of the orifice spacing in detail. These five samples all contain two adjacent orifices with a sizing equal to jet pump sample No. 3 (see Table 6.1). The mutual spacing is varied between $d_{h}=2.7 D_{\mathrm{s}}, 3.0 D_{\mathrm{s}}, 3.5 D_{\mathrm{s}}$, $4.0 D_{\mathrm{s}}$ and $5.0 D_{\mathrm{s}}$. The total cross-sectional area is identical for these five samples $\left(A_{s}=77.0 \mathrm{~mm}^{2}\right)$ but two times smaller than the samples discussed in Section 6.3.1. The dimensions are listed in Table 6.2. The two orifices are separate parts that can be inserted and removed from the jet pump sample. Hence, the orifices are exactly identical for the different samples in order to eliminate possible influences of the manufacturing process and the orifice spacing is the only parameter that is changed.

The dimensionless time-averaged pressure drop and acoustic power dissipation measured using samples No. 9-13 are averaged in the turbulent regime. The resulting values are shown in Fig. 6.7 as a function of the scaled orifice spacing $d_{h} / D_{s}$ and deliberately plotted on a similar vertical range as the results presented in Sec. 6.3.1. Although slight differences in performance can be observed, the influence of the orifice spacing is much less apparent than the effect of increasing the number of orifices. Consequently, the performance differences discussed in the previous section can be fully attributed to the miniaturization of the individual orifices and the effect of the orifice spacing is negligible, at least in the range of $d_{h} / D_{s}$ investigated. 

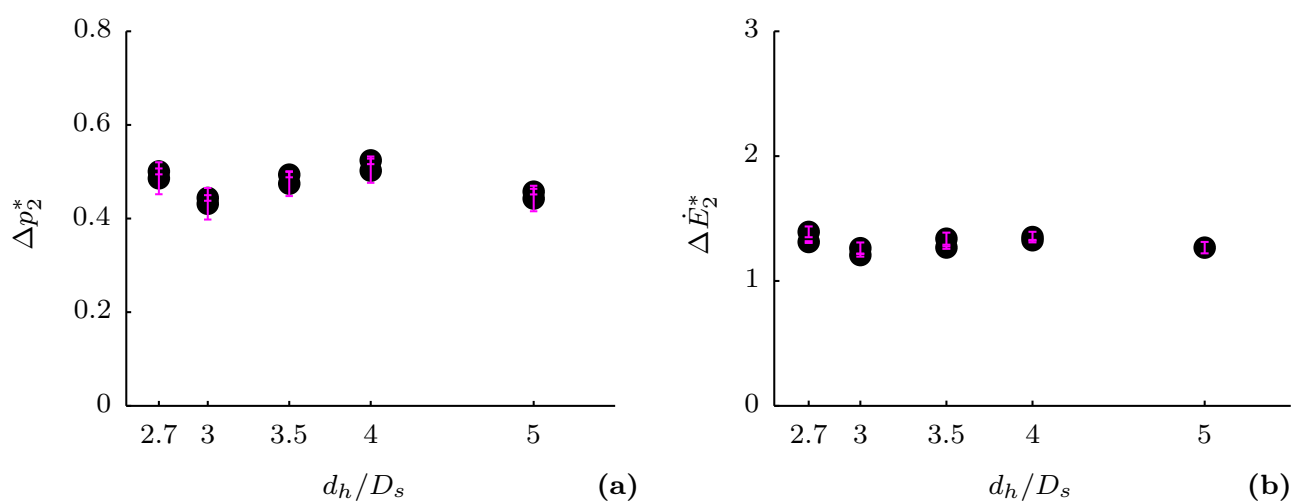

Figure 6.7: Averaged dimensionless pressure drop (a) and acoustic power dissipation (b) for various orifice spacing using samples No. 9-13. Error bars indicate the standard deviation of averaging.

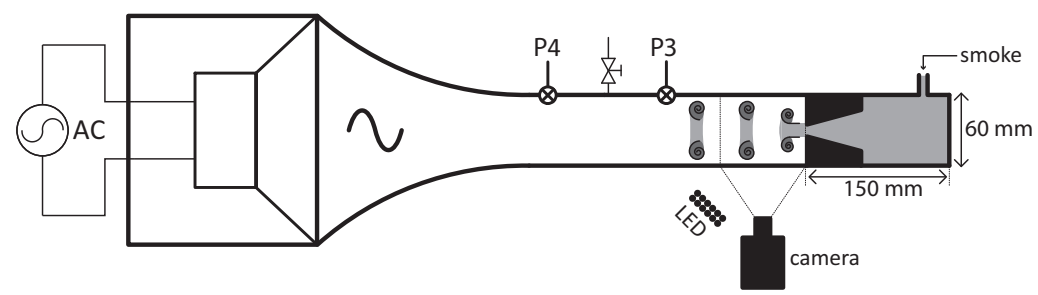

Figure 6.8: Schematic of experimental setup used for flow visualization purposes (not to scale). Compared with Fig. 6.1, the jet pump is installed in a reversed orientation and the tube length to the right of the jet pump is reduced.

\subsection{Flow visualization}

One of the questions that still remains, is how the flow field is influenced when the orifice spacing is varied. With the presented minor loss measurements and the literature on synthetic jets in mind, ${ }^{111,112}$ two possible answers are perceivable: either there are no differences at all in the vortex ring formation from the current jet pump samples, or the merging of vortex rings has no significant effect on the minor losses. The latter is the case, which is shown in the following using a flow visualization method.

The experimental setup configuration used for flow visualization purposes is illustrated in Fig. 6.8. Aerosol generated from a Dräger smoke tube is inserted in the experimental setup and acts as a tracer to visualize the air flow. For this purpose, the jet pump samples are placed in a reversed orientation in the setup (i.e., with the small opening facing the loudspeaker), and the tube length between the jet pump and the closed setup termination is reduced as shown in Fig. 6.8. This minimizes the volume to be filled with smoke and ultimately enhances the contrast of the images captured. The volume between the jet pump sample and the setup termination is continuously seeded with smoke. This generates a mean flow through the jet pump, which is coun- 


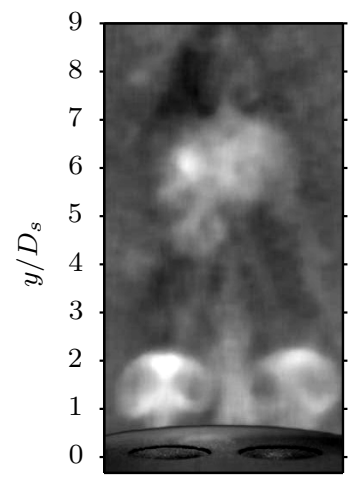

$t / T=0$

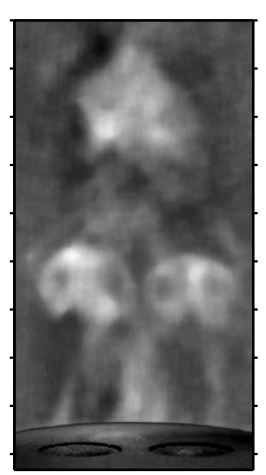

$t / T=0.4$

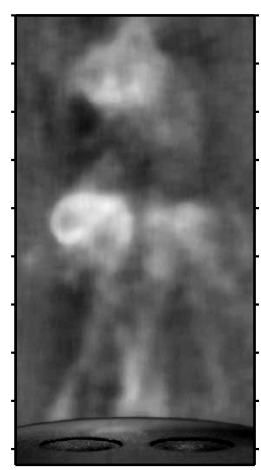

$t / T=0.6$

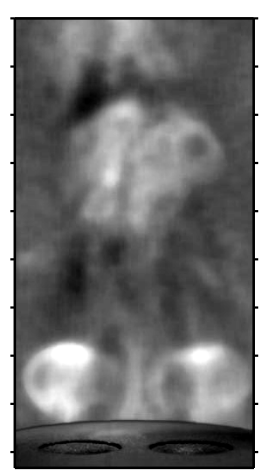

$t / T=1$

Figure 6.9: Visualization of vortex propagation from jet pump sample No. 9 with $d_{h} / D_{s}=2.7$ operated at $f=40 \mathrm{~Hz}$ and $K C_{D}=2$. At $t / T=0$, two vortex rings are formed and start propagating while moving towards each other $(t / T=0.4)$. The vortices ultimately merge $(t / T=0.6)$ and breakup in a less coherent structure $(t / T=1)$. Images are rotated $90^{\circ}$ with respect to Fig. 6.8 .

teracted by slightly pressurizing the volume at the other side of the jet pump. The flow exiting the small jet pump opening is captured using a Phantom v7.3 high-speed camera at $1000 \mathrm{fps}$, equipped with a Nikkor $40 \mathrm{~mm}$ macro lens. Using an aperture of $f / 3.6$, the estimated depth of field is $\pm 3.5 \mathrm{~mm}$ around the center plane of the jet pump. Illumination is provided by an LED panel placed at a $45^{\circ}$ horizontal angle to the test section as illustrated in Fig. 6.8. After recording, several post-processing steps are performed. The background (mean) image is subtracted, followed by applying a median filter for camera noise removal. Furthermore, the light intensity is normalized among the full range of images acquired to enhance the visibility of individual flow features.

Fig. 6.9 shows the evolution of vortex rings from jet pump sample No. 9 (with $d_{h} / D_{s}=$ 2.7) at $K C_{D}=2$ and a driving frequency of $40 \mathrm{~Hz}$. Note that the images are rotated $90^{\circ}$ with respect to Fig. 6.8. The jet pump sample is visible at the bottom of each image. At $t / T=0$, two separate vortex rings are formed from the individual jet pump holes and start propagating. While propagating, the vortex rings move towards each other (visible at $t / T=0.4$ ) and finally appear to merge to one vortex ring $(t / T=0.6)$. Soon after merging, the coherent vortex ring structure breaks apart and all that remains is a "blob" of smoke, which becomes indistinguishable from the smoke present in the setup.

Similar visualizations have been carried out for the other jet pump samples. Up to and including jet pump sample No. 11 (with $d_{h} / D_{s}=3.5$ ), the same flow regime is observed where the two vortex rings merge into one larger flow structure. However, for $d_{h} / D_{s}=4.0$ (sample No. 12), this is no longer the case and the vortex rings propagate freely without interaction. An example of this flow regime is shown in Fig. 6.10 at the same operating conditions as Fig. 6.9. This transition in the vortex flow regime corresponds well to the transitional $K C_{D}$ numbers found for synthetic jet 


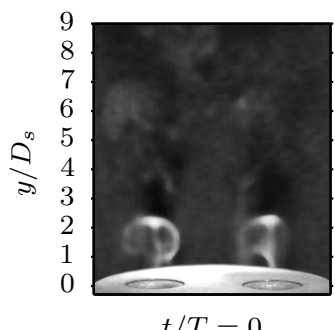

$t / T=0$

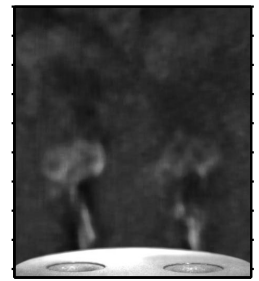

$t / T=0.4$

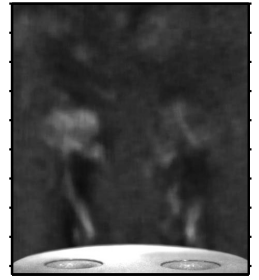

$t / T=0.6$

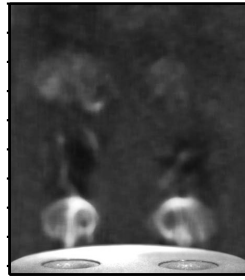

$t / T=1$

Figure 6.10: Visualization of vortex propagation from jet pump sample No. 12 with $d_{h} / D_{s}=4.0$ operated at $f=40 \mathrm{~Hz}$ and $K C_{D}=2$. At $t / T=0$, two vortex rings just emitted from jet pump and start propagating. No merging is observed during the full period and at $t / T=1$ a new set of vortex rings is visible. Images are rotated $90^{\circ}$ with respect to Fig. 6.8.

arrays. $^{111}$

The combination of flow visualization and pressure measurements shows that the merging of vortex rings does not have a significant effect on the minor losses occurring for orifice spacings as small as $d_{h} / D_{s}=2.7$. Whether minor losses are affected for $d_{h} / D_{s}<2.7$ cannot be evaluated with the current jet pump geometries and is left for future research. For $d_{h} / D_{s} \geq 2.7$, the losses generated at the jet pump waist remain approximately the same and no additional flow losses are induced when the two vortex rings merge. However, a point of discussion is the large difference between the values of $K C_{D}$ where the jet pump performance is quantified (Fig. 6.7) and where flow visualization is carried out. The current flow visualization method is not suitable for visualizing turbulent flows. However, we have carried out visualization at $K C_{D}=2$, 3 and 4 and no qualitative differences in the vortex merging were observed. The major difference between $K C_{D}=2$ and $K C_{D}=4$, is the point at which the vortex rings roll up after being expelled from the jet pump holes. That said, the vortex merging does not change noticeably. Furthermore, at $K C_{D}=2$ the differences in jet pump performance among the samples with varied orifice spacing is of the same order compared to the results shown in Fig. 6.7. This supports the deduced negligible effect of vortex merging on the overall jet pump performance.

\subsection{Conclusions}

For practical jet pump design, employing multiple parallel orifices is essential to achieve the required time-averaged pressure drop with a compact jet pump geometry. This study can serve as a first step towards a better understanding of the complications that might arise when using this jet pump design approach. The time-averaged pressure drop and acoustic power dissipation for jet pumps with 1 to 16 orifices and two different taper angles is measured. It has been shown that the dimensionless timeaveraged pressure drop $\Delta p_{2}^{*}$ decreases from 0.65 to 0.45 upon increasing the number of orifices. Rather than a direct effect of the number of holes, this performance drop can be attributed to a change in diameter of the individual orifices. A possible cause is 
the different relation between the onset of flow separation and the onset of turbulence when the orifice diameter is changed.

The effect of the mutual orifice spacing is investigated separately. Although differences in the vortex propagation are observed in the flow visualization, varying the orifice spacing between $2.7 \leq d_{h} / D_{s} \leq 5.0$ does not lead to a large change in the jet pump performance. Consequently, the merging of two vortex rings has no effect on minor losses for $d_{h} / D_{s} \geq 2.7$, which allows adjacent jet pump orifices to be placed close together. The performance penalty of employing multiple parallel orifices is most likely caused by the miniaturization of the individual orifices rather than by the interaction of the flows from adjacent orifices.

Although there is a significant performance decay between the 1 and 16 hole jet pumps, it is important to realize that this decay bears no relation to the low performance that is expected when the single hole jet pump size would be decreased by reducing its length, thus increasing the taper angle. ${ }^{85}$ An equal decrease in length which is realized in this study by employing 16 holes, would result in a single hole taper angle of $36^{\circ}$ for the current jet pump cross-sectional areas. This is way beyond a taper angle at which any practical jet pump performance is to be expected. ${ }^{85}$ Hence, designing jet pumps with multiple parallel orifices is the most promising approach for compact designs and the optimal compromise between jet pump performance and compactness should be evaluated for each particular design.

All in all, a clear effect of the number of holes on the jet pump performance is observed but more research is required to decisively pinpoint the physical phenomena that lead to this effect before any design guidelines can be formulated. 


\section{Conclusions}

The work presented in this thesis serves as a step towards a better understanding of the oscillatory flow phenomena in jet pumps with a focus on deriving practical design guidelines for thermoacoustic applications. After summarizing the main conclusions from the individual chapters in Sections 7.1-7.4, a more general outlook is provided in Section 7.5 summarizing the key results and discussing recommendations for further research.

\subsection{Jet pump performance in laminar oscillatory flows}

For laminar oscillatory flows, the jet pump performance is determined using an axisymmetric transient CFD model of a single hole jet pump. The dimensionless timeaveraged pressure drop and the dimensionless acoustic power dissipation across the jet pump are calculated for a range of jet pump geometries and wave amplitudes. The geometries span a range of taper angles, diameters and radii of curvature. The computational model is validated experimentally for laminar oscillatory flows using both a $7^{\circ}$ and $15^{\circ}$ taper angle jet pump.

Different flow regimes are distinguished and their impact on the jet pump performance is investigated. It is found that the occurrence of vortex rings propagating from the small hole of the jet pump correlates with an increase in the dimensionless timeaveraged pressure drop for all geometries considered. The onset of vortex shedding from the jet pump small hole is determined by the Keulegan-Carpenter number and occurs when $K C_{D}>0.5$ for jet pumps with a dimensionless waist curvature of $\chi=$ $R_{c} / D_{s}=0.36$. For sharp edges (i.e., $\chi=0$ ), this formation criterion reduces to $K C_{D}=0.15$, which corresponds to numbers reported for a sharp-edged synthetic jet. ${ }^{68}$ Two different expressions are proposed to include the effect of waist curvature in the formation criterion, but the data available is inconclusive to choose one or the other. An increase in curvature clearly results in the onset of flow separation (see Sec. 7.2) being shifted to higher displacement amplitudes. Nevertheless, a larger curvature also leads to a reduction in the expansion minor loss coefficient which is key in generating the time-averaged pressure drop. 
Similarly, the onset of vortex propagation from the jet pump big opening can be related to the local Keulegan-Carpenter number at that side of the jet pump. Because the big hole is generally sharp-edged, vortex rings are shed when $K C_{D, b}>0.15$. However, as the local velocities are low compared to the velocity at the jet pump waist, these vortex rings are much weaker and the related minor losses are negligible for the geometries considered in this study.

\subsection{Characterization and reduction of flow separation}

Next to the flow regimes determined by vortex shedding from either of the two jet pump openings, another flow regime is distinguished. This flow regime occurs when the flow separates from the inner jet pump wall during the diverging flow direction. The adverse pressure gradient in the diverging flow direction can result in flow reversal close to the jet pump wall. The contracting bulk flow at the jet pump waist subsequently forces the reversed flow to recirculate. This induces breakaway of the boundary layer and a stream of vortices to occur in the diverging flow direction. The wake formation process is associated with additional minor losses in the diverging flow direction, which counteracts the minor losses generated by the vortex propagation from the jet pump small hole and results in a significant decrease of the dimensionless time-averaged pressure drop. Furthermore, the overall acoustic power dissipation is increased by these additional minor losses. As such, the flow separation is highly undesirable for efficient jet pump operation and should be avoided.

In absence of turbulent flow effects, the onset of flow separation is fully determined by a combination of the dimensionless displacement amplitude $K C_{D}$ and the taper angle: $K C_{\alpha}=\xi_{1} / D_{s} \cdot \alpha$. For the jet pump geometries investigated with $\chi=0.36$, flow separation is found to occur for $K C_{\alpha}>0.7$. This onset is confirmed experimentally using hot-wire anemometry for jet pumps with a $7^{\circ}$ and $15^{\circ}$ taper angle.

A measure to avoid flow separation is the use of a low taper angle. However, this leads to a larger jet pump length, which is not always possible within the design space available. Hence, an alternative way to reduce the adverse pressure gradient in the vicinity of the flow separation point is proposed. A more smooth, circular transition between the jet pump waist and the linear tapered wall is introduced, that acts as a local reduction of the taper angle. Six different geometries are investigated, characterized by the extra transition length $\Delta L_{J P}$ introduced with respect to the reference case. The smooth transition results in a shift of the onset of flow separation towards higher values of $K C_{D}$. When using a smooth transition of $\Delta L_{J P}=20 \mathrm{~mm}$, the displacement amplitude at which the flow first separates is increased by more than a factor of two. Furthermore, the flow is separated for a shorter time each period and the location of the first flow reversal point is shifted to a larger local radius. This results in an increase in the jet pump performance as the losses due to the wake formation are reduced. Additionally, the range of $K C_{D}$ with effective jet pump performance is enlarged, which results in a more robust design. 


\subsection{Influence of turbulence on the jet pump performance}

While the jet pump performance in laminar oscillatory flows is mainly characterized numerically, an experimental investigation has been carried out to determine the effect turbulence can have on the performance of jet pumps. Using hot-wire anemometry the acoustic Reynolds number at the jet pump waist is shown to be a good predictor for the occurrence of turbulence in the oscillatory flow in jet pumps. As the Reynolds number approaches its critical value for oscillatory pipe flow, the power spectra start following a Kolmogorov $-5 / 3$ power law decay. Furthermore, for $R e>R e_{c}$ hysteresis is found in the phase-averaged standard deviation of the velocity. This can be explained by the onset of turbulence and the subsequent relaminarization that occurs every wave period.

Good correspondence is found between the simulated and measured dimensionless time-averaged pressure drop in the laminar regime. However, in the turbulent regime a different behavior is observed as the time-averaged pressure drop has the tendency to stabilize rather than to decrease continuously, as observed for laminar flow. Furthermore, for a fixed value of $K C_{\alpha}$ the dimensionless acoustic power dissipation shows a decaying trend as a function of $R e$. These two observations support the hypothesis that the negative effect flow separation has on the jet pump performance is reduced in the turbulent regime, which is in line with literature on flow separation in steady flows. ${ }^{92}$

\subsection{Jet pumps with multiple orifices}

In order to design compact jet pumps, the taper angle can be increased. However, this research shows that this can easily lead to flow separation and a reduced jet pump performance. An alternative approach to a more compact jet pump is distributing the total cross-sectional area over multiple smaller orifices. Configuring the single hole $7^{\circ}$ jet pump sample used in this research in a 16 holes design, reduces the total length from $70 \mathrm{~mm}$ to $8.8 \mathrm{~mm}$. However, the dimensionless pressure drop in the turbulent regime decreases from 0.65 to 0.45 while the dimensionless acoustic power dissipation more than doubles from 1.2 to 2.6 when comparing a single hole jet pump to a jet pump having 16 parallel orifices.

Flow visualization of vortex rings from adjacent jet pump orifices shows that depending on the orifice spacing, the adjacent vortex rings can merge into one larger flow structure. However, by varying merely the orifice spacing no significant change in jet pump performance is measured, which suggests that the merging of vortex rings has no effect on the minor losses in the range of orifice spacings investigated. Consequently, the measured performance decay for jet pumps with multiple orifices can be attributed to the smaller orifice diameter rather than to the interaction of flows from adjacent orifices. This can be understood as both the onset of turbulence (determined by $R e_{c}$ ) as well as the onset of flow separation (determined by $K C_{\alpha}$ ) are influenced by the individual orifices having a smaller diameter. This emphasizes the importance 
of turbulence and flow separation on a jet pump's performance.

\subsection{Outlook}

This research attempts to provide a better physical understanding of the oscillatory flow phenomena occurring in jet pumps for thermoacoustic applications. For the first time, flow separation is directly identified as a main source of performance loss in jet pumps. Especially in laminar oscillatory flows, the effect is severe for all jet pump taper angles investigated. In turbulent flows, the flow separation is slightly suppressed. However, still large performance penalties are measured for higher taper angles such as those required for compact jet pump applications. To overcome this, a design alteration is proposed to reduce flow separation and improve the effectiveness and robustness of jet pumps while still allowing for a compact design. Furthermore, the performance of jet pumps with multiple orifices is quantified. While distributing the cross-sectional area over multiple smaller orifices can lead to highly compact designs, this approach leads to a performance reduction compared to the single hole jet pumps. Although this is a significant effect, making a single hole jet pump more compact by just increasing its taper angle will lead to a much more severe performance drop. Designing jet pumps with multiple parallel orifices is the most promising approach for compact designs.

The surface roughness of the inner jet pump wall is not investigated in much detail in this work. A preliminary study (Appendix D) shows that differences in the jet pump performance can be expected as a result of a change in surface roughness. Further research should identify whether surface roughness is merely a parameter that needs to be controlled, or that smart local roughness adaptations can eventually become an additional degree of freedom in the design of jet pumps.

Another aspect that requires further attention, is the impact of a jet pump on the acoustic field inside a thermoacoustic device. Although previous research has shown that the impedance of jet pumps is highly non-linear, ${ }^{60}$ the direct effect of the oscillatory flow regimes on the acoustic quantities is not investigated and further research is required to examine this. Moreover, the impact of the jet pump flow on adjacent components in a thermoacoustic application is open to future research.

In addition to the applicability of the current research to jet pumps, the results presented in this work could possibly act as a guide to identify oscillatory flow problems in similar components such as diffusers, expansions, orifices or synthetic jet actuators. The numerical approach developed as well as the applied experimental methods are also suitable for these kinds of oscillatory flow applications. Similarities in results for diffusers, synthetic jets and jet pumps are observed, especially in the onset of flow phenomena such as vortex formation and flow separation. Drawing parallels between these applications is essential to yield a comprehensive knowledge of the underlying fluid dynamics. Understanding the impact a jet pump has on the system level together with the local flow phenomena identified in the current work, will result in more reliable design calculations for jet pumps in thermoacoustic applications. 


\section{APPENDICES}

A Time-domain impedance boundary condition $\quad 101$

A.1 Introduction . . . . . . . . . . . . . . . . . . . . . 101

A.2 Theory and implementation . . . . . . . . . . . . . . . 102

A.2.1 Non-reflective boundary condition . . . . . . . . . . . . . . . 102

A.2.2 Time-domain impedance boundary condition . . . . . . . . . . . . 104

A.2.3 Implementation . . . . . . . . . . . . . . . . . . . . . . . . . . . . . 104

A.3 One-dimensional validation . . . . . . . . . . . . . . . . . . . . . 104

A.4 Two-dimensional validation . . . . . . . . . . . . . . . . . 107

A.4.1 Low reduced frequency model . . . . . . . . . . . . . . 107

A.4.2 Numerical setup . . . . . . . . . . . . . . . . . . . . . . 108

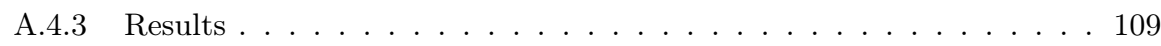

B Pressure measurement and calibration 111

B.1 Signal sampling . . . . . . . . . . . . . . . . . . . . . 112

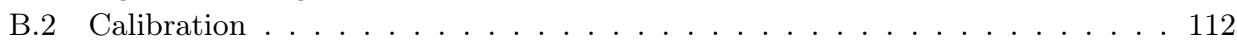

B.2.1 Sensor sensitivity . . . . . . . . . . . . . . . 113

B.2.2 Phase correction . . . . . . . . . . . . . . . . 1114

B.2.3 Linearity . . . . . . . . . . . . . . . . . . . . . 115

B.2.4 Stability and repeatability . . . . . . . . . . . . 116

C Acoustic modeling of experimental setup 117

C.1 Introduction . . . . . . . . . . . . . . . . . 117

C.2 Model . . . . . . . . . . . . . . . . . . . . . 117

C.2.1 Low reduced frequency model . . . . . . . . . . . . . . . . 118

C.2.2 Coupled COMSOL model . . . . . . . . . . . . . . . . . 119

C.2.3 From measured pressure to velocity amplitude . . . . . . . . . . . . . 119

C.3 Results . . . . . . . . . . . . . . . . . . . . 120

C.3.1 Effect of jet pump geometry . . . . . . . . . . . . . . 122

C.4 Discussion . . . . . . . . . . . . . . . . . . . 123

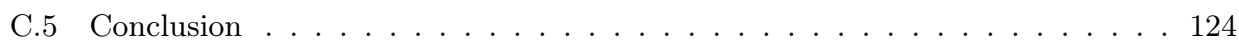

$\begin{array}{lr}\text { D Surface roughness } & 125\end{array}$

D.1 Introduction . . . . . . . . . . . . . . . . . . . . . 125

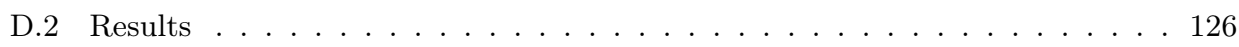

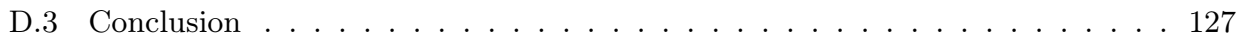




\section{APPENDIX}

\section{Time-domain impedance boundary condition}

\section{A.1 Introduction}

For the application to the jet pump simulations carried out in Chapters 2-4, a boundary condition has been developed that is capable of simulation traveling wave conditions in the computational domain. This filter-based time-domain impedance boundary condition is similar to the approach proposed by Polifke et al. ${ }^{80,81}$ and is implemented in ANSYS CFX (see Section A.2).

Although it has been found experimentally that the wave phasing does not play a major role in the jet pump performance, ${ }^{91}$ another motivating factor for the boundary condition development is the absorption of propagating flow structures such as vortex rings, which greatly reduces the time required to reach a steady periodic solution. Next to this, the presented boundary condition can also be used to model other components of a (thermo)acoustic system. By imposing the correct acoustic impedance at the boundaries, the flow in a single component can be evaluated without simulating the complete system.

In order to validate the implemented time-domain impedance boundary condition in ANSYS CFX, several cases of wave propagation have been simulated. First of all, one-dimensional wave propagation has been studied in Section A.3. The resulting reflection coefficient is compared to the specified reflection coefficient for different values of the pressure amplitude. Moreover, the drift of mean pressure over time has been calculated. In a second study (Section A.4), the two-dimensional wave propagation in a tube has been studied. The simulation results are compared to the low-reduced frequency wave propagation model. ${ }^{82}$ 


\section{A.2 Theory and implementation}

In this section, a brief description of the underlying theory and numerical implementation of the time-domain impedance boundary condition is provided. More details are available in the work of Van der Poel, which has been carried out as part of the current research. ${ }^{79}$ The complete implementation consists of several parts:

- A non-reflective part based on the locally one-dimensional inviscid (LODI) formulation of the Navier-Stokes equations.

- A linear relaxation method (LRM) to avoid stability issues.

- A plane-wave masking (PWM) term to reduce additional reflections introduced by the LRM.

- A time-domain impedance boundary condition (TDIBC) specific part.

ANSYS CFX also offers a non-reflecting boundary condition as an inbuilt "beta feature". ${ }^{114}$ However, preliminary simulations with jet pump geometries resulted in numerical instabilities when vortices reached the boundary condition. The plane wave masking approach implemented in the current time-domain impedance boundary condition has solved this issue.

\section{A.2.1 Non-reflective boundary condition}

The non-reflective part is based on the locally one-dimensional inviscid (LODI) formulation of the Navier-Stokes equations. The Navier-Stokes equations are rewritten in characteristic form and the transverse and viscous terms are dropped. This results in a system of equations describing the amplitude variations of the characteristic waves,

$$
\begin{aligned}
\frac{\partial \rho}{\partial t}+\frac{1}{c^{2}}\left(\mathcal{L}_{2}+\frac{1}{2}\left(\mathcal{L}_{5}+\mathcal{L}_{1}\right)\right) & =0, \\
\frac{\partial p}{\partial t}+\frac{1}{2}\left(\mathcal{L}_{5}+\mathcal{L}_{1}\right) & =0, \\
\frac{\partial u}{\partial t}+\frac{1}{2 \rho c}\left(\mathcal{L}_{5}-\mathcal{L}_{1}\right) & =0, \\
\frac{\partial v}{\partial t}+\mathcal{L}_{3} & =0, \\
\frac{\partial w}{\partial t}+\mathcal{L}_{4} & =0,
\end{aligned}
$$


from which $\mathcal{L}_{i}$ is defined as

$$
\begin{aligned}
\mathcal{L}_{1} & =\lambda_{1}\left(\frac{\partial p}{\partial x}-\rho c \frac{\partial u}{\partial x}\right) \\
\mathcal{L}_{2} & =\lambda_{2}\left(c^{2} \frac{\partial \rho}{\partial x}-\frac{\partial p}{\partial x}\right) \\
\mathcal{L}_{3} & =\lambda_{3} \frac{\partial v}{\partial x} \\
\mathcal{L}_{4} & =\lambda_{4} \frac{\partial w}{\partial x} \\
\mathcal{L}_{5} & =\lambda_{5}\left(\frac{\partial p}{\partial x}-\rho c \frac{\partial u}{\partial x}\right)
\end{aligned}
$$

with $c$ the local speed of sound. The eigenvalues or characteristic velocities are

$$
\begin{aligned}
& \lambda_{1}=u_{1}-c, \\
& \lambda_{2}=\lambda_{3}=\lambda_{4}=u_{1}, \\
& \lambda_{5}=u_{1}+c .
\end{aligned}
$$

$\mathcal{L}_{1}$ enters the domain at an outlet and needs to be specified while $\mathcal{L}_{2 \ldots 5}$ are calculated from the flow field. A fully non-reflective boundary condition means setting $\mathcal{L}_{1}=0$. However, this can lead to an ill-posed condition as no information about the the farfield pressure is able to propagate to the interior of the domain. A solution to this stability problem is to apply a linear relaxation method as described by Poinsot and Lele. ${ }^{115}$ This method effectively restores a deviation from the target far-field pressure $p_{\infty}$

$$
\mathcal{L}_{1}=K\left(p-p_{\infty}\right)
$$

However, this additional term will introduce reflections. The choice of the relaxation factor $K$ is a compromise between pressure drift and acoustic reflection and needs to be chosen with care. ${ }^{116}$ To reduce these additional reflections, plane-wave masking can be applied. ${ }^{117}$ This approach determines the amplitude of the characteristic wave $f$ at a sample plane inside the computational domain. By shifting $f$ in time and subtracting from the LRM term (Eq. A.14),

$$
\mathcal{L}_{1}=K\left(p-p_{\infty}-\rho_{0} c_{0} f\right)
$$

the acoustic pressure oscillation is "masked" and the reflections are greatly reduced while maintaining numerical stability. The amplitude of the characteristic wave $f$ is derived from the numerical solution by area-averaging the pressure and velocity at the sample plane and subtracting the known mean values $p_{0}$ and $u_{0}$,

$$
f=\frac{1}{2}\left(\frac{\overline{p-p_{0}}}{\rho_{0} c_{0}}+\overline{u-u_{0}}\right) .
$$




\section{A.2.2 Time-domain impedance boundary condition}

The non-reflective boundary condition introduced in Section A.2.1 can be extended to specify any desired complex reflection coefficient. ${ }^{81}$ An external perturbation $g^{*}$ is added to the existing equation for $\mathcal{L}_{1}$,

$$
\mathcal{L}_{1}=K\left(p-p_{\infty}-\rho_{0} c_{0}\left(f+g^{*}\right)\right)-\rho_{0} c_{0} \frac{\partial g^{*}}{\partial t} .
$$

The goal is to calculate this external perturbation $g^{*}$ such that it mimics exactly the desired complex reflection coefficient $\mathcal{R}(\omega)$. Given the definition of the reflection coefficient $\mathcal{R}(\omega)=g(\omega) / f(\omega)$, the backward traveling characteristic wave amplitude $g(\omega)=\mathcal{R}(\omega) f(\omega)$ is well defined in the frequency domain. To be able to arrive at an expression in the time-domain, a discrete Laplace transformation is applied and the reflection coefficient is approximated by a transfer function of the form ${ }^{81}$

$$
\mathcal{R}(z)=\frac{a_{0}+a_{1} z^{-1}+a_{2} z^{-2}+\cdots+a_{n} z^{-n}}{1-b_{1} z^{-1}+b_{2} z^{-2}+\cdots+b_{m} z^{-m}} .
$$

This finally yields an equation for the external perturbation, ${ }^{81}$

$$
g^{*}(t)=\sum_{i=0}^{n} a_{i} f(t-i \Delta t)-\sum_{j=1}^{m} b_{j} g(t-j \Delta t) .
$$

The filter coefficients $a_{i}$ and $b_{j}$ are determined from the specified $\mathcal{R}(\omega)$ using complex curve fitting algorithms. More details on the determination of the filter coefficients and their stability can be found in the work of Van der Poel. ${ }^{79}$ For all computational studies presented in Chapters 2-4, a frequency independent reflection coefficient of $\mathcal{R}=0$ is used (fully non-reflective). This strongly simplifies the situation and sets all filter coefficients to zero.

\section{A.2.3 Implementation}

The aforementioned methods are implemented in the CFD software package ANSYS CFX using several FORTRAN subroutines. Ultimately, the pressure at the boundary is imposed from Eq. A.2 where $\mathcal{L}_{1}$ is determined from Eq. A.17. A Crank-Nicolson scheme is used for time-integrating $\partial p / \partial t$ and $\mathcal{L}_{5}$ is determined from the sample plane inside the computational domain. More details on the data structure and memory management can be found in the work of Van der Poel. ${ }^{79}$

\section{A.3 One-dimensional validation}

A computational domain in ANSYS CFX with a length of $L=1 \mathrm{~m}$ has been used to investigate the performance of the time-domain impedance boundary condition in one-dimensional wave propagation. On the left and right side of the domain, a timedomain impedance boundary condition is used. An incoming wave is imposed by 
adding an external pressure perturbation with amplitude $\left|p_{1}\right|_{\text {spec }}$ to the time-domain impedance boundary condition on the left side of the domain. On the right side of the domain, a complex reflection coefficient is specified. A uniform computational mesh with 1000 elements in the wave propagation direction is used $(\Delta x=1 \mathrm{~mm})$ and a total of 10 wave periods is simulated using a time-step of $\Delta t=1 \cdot 10^{-5} \mathrm{~s}$. The last 5 wave periods are used to calculate the results shown. The wave sample planes for the time-domain impedance boundary condition are placed at a distance of $20 \mathrm{~mm}$ from the boundaries. Helium is used as a working fluid at a reference temperature of $T_{0}=298.15 \mathrm{~K}$ and a reference pressure of $p_{0}=1 \mathrm{~atm}$.

The deviation from the specified reflection coefficient has been calculated using

$$
\Delta R=R-R_{\text {spec }},
$$

where $\mathcal{R}$ is the resulting complex reflection coefficient at the right sided time-domain impedance boundary condition and is calculated using

$$
\mathcal{R}=\frac{p^{-}}{p^{+}}
$$

The amplitude of the forward traveling wave $p^{+}$and the backward traveling wave $p^{-}$ are calculated from

$$
\begin{aligned}
& p^{+}=\frac{1}{2}\left(p_{1}+\rho_{0} c_{0} u_{1}\right) \\
& p^{-}=\frac{1}{2}\left(p_{1}-\rho_{0} c_{0} u_{1}\right) .
\end{aligned}
$$

Two different cases have been simulated: one fully non-reflecting case $\left(\mathcal{R}_{\text {spec }}=0+0 i\right)$ and one case simulating a partially reflecting outlet $\left(\mathcal{R}_{\text {spec }}=0.5+0.5 i\right)$. For both cases, five different pressure amplitude levels have been simulated: $\left|p_{1}\right|=0.2 \mathrm{kPa}$, $2 \mathrm{kPa}, 4 \mathrm{kPa}, 8 \mathrm{kPa}$ and $16 \mathrm{kPa}$ at a constant driving frequency of $f=100 \mathrm{~Hz}$.

In Table A.1 the results of all one-dimensional simulations are listed. For the nonreflective case, the measured reflection coefficient at the right side of the domain remains low $(\mathcal{R}<1 \%$ ) up to a pressure drive ratio of $16 \%$. The drift of mean pressure after ten wave periods, $p_{2}$, shows a quadratic relation to the pressure amplitude $\left|p_{1}\right|$. Hence, $p_{2} /\left|p_{1}\right|$ increases linearly with $\left|p_{1}\right|$. This will lead to an error in the calculated second order pressure field, depending on the number of wave periods used to calculate this field. If, for example, the second order quantities are calculated using the last two periods of the simulation, the estimated error at a drive ratio of $\mathrm{Dr}=8 \%$ is $\varepsilon_{p_{2} / p_{1} \mid} \approx 8.74 \% \cdot \frac{2}{10}=1.75 \%$, which is considered acceptable. The results of the partially reflecting case show in general higher deviations from the specified reflection coefficient and higher pressure drift.

Besides the specified reflection coefficient, the influence of different other simulation parameters has been studied. Both simulations using air and helium as a working fluid have been carried out. Furthermore, the spatial discretization has been varied 


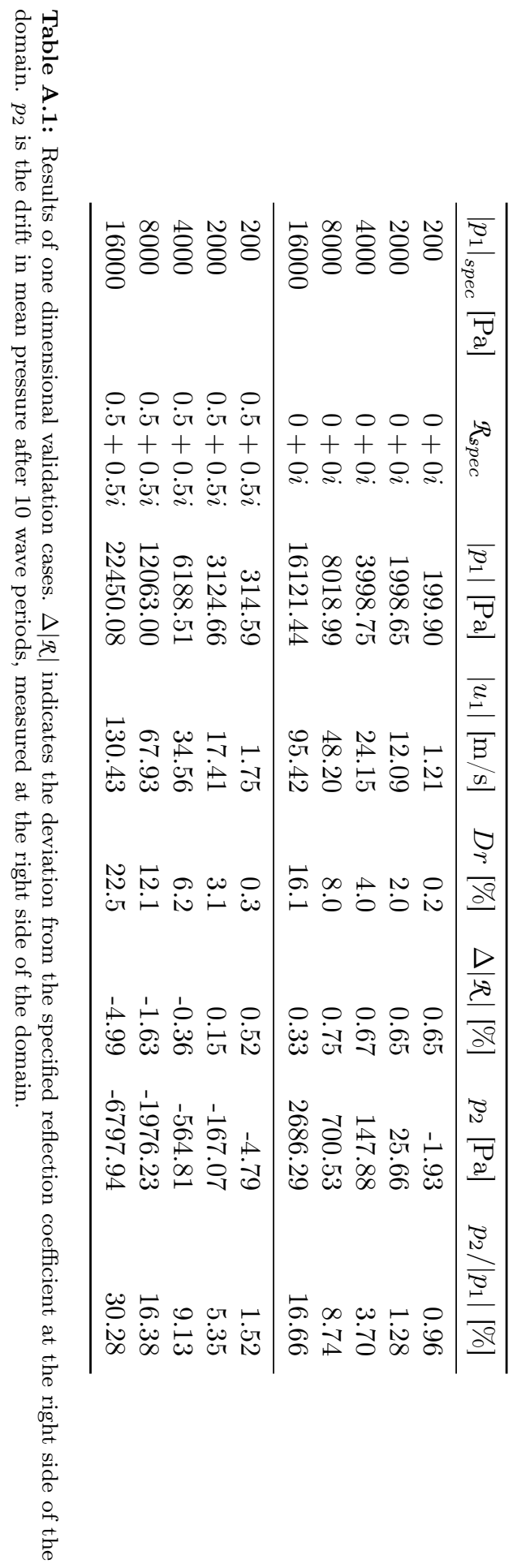


between $\Delta x=1 \mathrm{~mm}$ and $\Delta x=4 \mathrm{~mm}$ and the domain length between $L=1 \mathrm{~m}$ and $L=4 \mathrm{~m}$. In all cases, no significant effect on the simulation results was observed. Moving the location of the wave sample plane closer to the boundary does have a slight positive effect on $\Delta \mathcal{R}$. Changing from $400 \mathrm{~mm}$ to $20 \mathrm{~mm}$ leads to a decrease in $\Delta \mathcal{R}$ of approximately $0.3 \%$ for the non-reflecting case.

\section{A.4 Two-dimensional validation}

The low-reduced frequency model provides an analytic solution to the two-dimensional wave propagation inside a tube. ${ }^{82}$ By comparing this solution to the $2 \mathrm{D}$ axisymmetric simulation results of wave propagation inside a tube, the flow fields can be compared in detail and the accuracy of simulations using ANSYS CFX in combination with the implemented time-domain impedance boundary condition can be determined.

\section{A.4.1 Low reduced frequency model}

Starting from the Navier-Stokes equations, the low-reduced frequency solution can be derived upon making the following assumptions:

- Linear harmonic oscillations.

- Tube radius small in comparison with wavelength $\left(\omega R_{0} / c_{0} \ll 1\right)$.

- Tube long enough such that end effects are negligible.

- Radial velocity small compared to axial velocity $(v / u \ll 1)$.

- No steady flow.

The two-dimensional low-reduced frequency solution in dimensionless coordinates $\xi=$ $\omega x / c_{0}$ and $\eta=r / R_{0}$ is given by ${ }^{82}$

$$
\begin{aligned}
p_{1} / p_{0} & =A e^{\Gamma \xi}+B e^{-\Gamma \xi}, \\
u_{1} / c_{0} & =\frac{i \Gamma}{\gamma}\left[1-\frac{J_{0}\left(i^{3 / 2} \eta s\right)}{J_{0}\left(i^{3 / 2} s\right)}\right]\left[A e^{\Gamma \xi}+B e^{-\Gamma \xi}\right],
\end{aligned}
$$

with $p_{1}, u_{1} \in \mathbb{C}$ the pressure and velocity amplitudes and $p_{0}$ the specified reference pressure. $A$ and $B$ are boundary conditions to be defined (see Eq. A.28) and $s$ is the shear wave number determined by $s=R_{0} \sqrt{\rho_{0} \omega / \mu}$ and is equivalent to the Stokes number. The wave propagation constant $\Gamma$ is calculated using

$$
\Gamma=\sqrt{\frac{J_{0}\left(i^{3 / 2} s\right)}{J_{2}\left(i^{3 / 2} s\right)}} \sqrt{\frac{\gamma}{n}},
$$

with $n$ a polytropic constant given by

$$
n=\left[1+\frac{\gamma-1}{\gamma} \frac{J_{2}\left(i^{3 / 2} \sigma s\right)}{J_{0}\left(i^{3 / 2} \sigma s\right)}\right]^{-1}, \quad \text { and } \quad \sigma=\sqrt{P r} .
$$




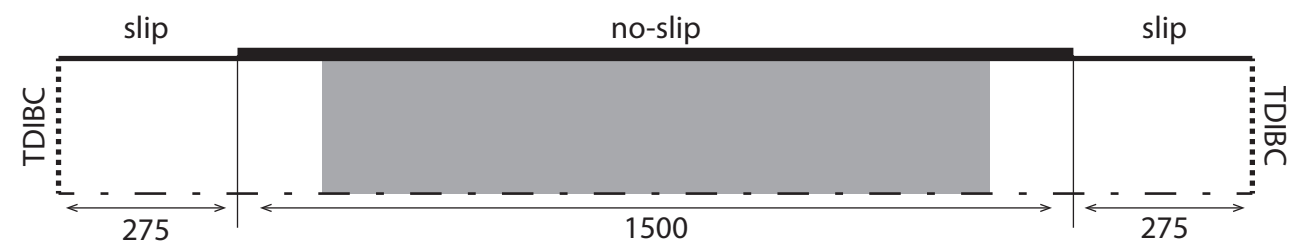

Figure A.1: Numerical setup for 2D validation of time-domain impedance boundary condition (not to scale). Thick black line indicates full region with no-slip wall boundary condition while the gray area is the region where the actual comparison is made between numerical results and results from the low reduced frequency model to avoid the influence of entrance effects.

\section{A.4.2 Numerical setup}

For the two-dimensional validation, a tube is simulated with on the left and right side time-domain impedance boundary conditions identical to what is stated in Section A.3. Because the theory behind the time-domain impedance boundary condition is based on one-dimensional adiabatic wave propagation, using a no-slip isothermal wall close to the time-domain impedance boundary conditions will lead to large errors as this will void the assumptions whereupon the time-domain impedance boundary condition is based. To overcome this, a slightly different configuration is used as illustrated in Fig. A.1. An adiabatic slip wall boundary condition with length $L_{\text {slip }}$ is used close to the time-domain impedance boundary conditions. In the center of the domain, an isothermal no-slip wall is imposed with length $L_{N S}=L-2 \cdot L_{\text {slip }}$. This results in a solution with one-dimensional wave propagation close to both time-domain impedance boundary conditions and, after a transition region, to results corresponding to $2 \mathrm{D}$ wave propagation in the center of the domain. It is in this last region where a comparison is made between the simulation results and the low-reduced frequency solution. The length of the simulated tube is $L=2050 \mathrm{~mm}$, the radius is $R_{0}=30 \mathrm{~mm}$. After a preliminary study it was decided to use a no-slip wall with a length of $L_{N S}=1500 \mathrm{~mm}$ centered in axial direction. This leads to slip walls on either side of the domain with a length of $L_{\text {slip }}=275 \mathrm{~mm}$ each.

In order to simulate axisymmetric conditions in ANSYS CFX, a 3D mesh needs to be generated which is extruded one element in azimuthal direction and symmetry boundary conditions are applied on the faces normal to the azimuth. For the current study, a computational mesh is used with $\Delta x=10 \mathrm{~mm}$. In radial direction, the mesh is refined towards the solid boundary such that a total number of $N_{B L}=10$ elements reside within one viscous penetration depth $\delta_{\nu}$ from the wall. Subsequently, the mesh nodes are placed using a geometric distribution towards the axis up to a maximum element size of $\max (\Delta r)=5 \mathrm{~mm}$. The wave sample plane for the timedomain impedance boundary conditions is placed at $50 \mathrm{~mm}$. The other conditions are similar to the one-dimensional validation case: 10 wave periods are simulated using a time-step of $\Delta t=1 \cdot 10^{-5} \mathrm{~s}$ and helium at STP conditions is used as a working fluid. All results are calculated using the last 5 simulated periods. Four different pressure amplitude levels have been simulated: $\left|p_{1}\right|=0.2 \mathrm{kPa}, 1 \mathrm{kPa}, 2 \mathrm{kPa}$ and $5 \mathrm{kPa}$ at a constant driving frequency of $f=100 \mathrm{~Hz}$. 
The transition from the slip wall to the no-slip wall leads to a local increase in the reflection coefficient and consequently a small standing wave component inside the no-slip region. Besides that, the entrance effects at both slip to no-slip transitions need to be taken into account when calculating the low-reduced frequency solution. To compensate for these effects, the low-reduced frequency solution is only calculated in a part of the no-slip region and the effective no-slip length (gray area in Fig. A.1) is reduced by $125 \mathrm{~mm}$ on either side. By calculating the boundary conditions $A$ and $B$ for the low-reduced frequency model from the local simulation results and solving the pressure equation at these compensated locations, the effect of the slip to noslip transition is correctly taken into account in the low-reduced frequency solution,

$$
\left[\begin{array}{cc}
1 & 1 \\
\exp \left(\Gamma \xi_{R}\right) & \exp \left(-\Gamma \xi_{R}\right)
\end{array}\right]\left\{\begin{array}{l}
A \\
B
\end{array}\right\}=\left\{\begin{array}{c}
\left(p_{1} / p_{0}\right)_{L} \\
\left(p_{1} / p_{0}\right)_{R}
\end{array}\right\},
$$

with $\xi_{L}=0$ substituted. The subscripts $L$ and $R$ determine the left and right side of the compensated no-slip wall region, respectively. The complex pressure amplitudes at these boundaries are determined from the numerical results using a discrete Fourier transform, identical to what is used in Chapters 2-4. All other physical parameters required for the calculation of the low-reduced frequency solution are derived from the initialization of the ANSYS CFX solver.

\section{A.4.3 Results}

In Figure A.2 the radial distribution of the axial velocity component $u$ is shown for four different time instances and at four different axial locations using a specified pressure amplitude of $\left|p_{1}\right|=5 \mathrm{kPa}$. It is clear that the simulation results do match with the low-reduced frequency solution at all locations and time instances shown. Note that only the region up to $10 \cdot \delta_{\nu}$ from the solid boundary is shown, outside this region the results do match as well. Similar results are obtained with lower pressure amplitudes.

In Figure A.3 the complex velocity and pressure amplitudes are compared against the low-reduced frequency solution. Because of the used method to determine the boundary conditions for the low-reduced model (see Eq. A.28), the left and right points of the pressure amplitude distribution will always match. The fact that the pressure amplitude distribution does match along the whole no-slip region, shows that the wave propagation constant $\Gamma$ does match with the low-reduced frequency solution and the time-domain impedance boundary condition does not cause any significant error in the wave propagation. The slight shift in the velocity magnitude $\left|u_{1}\right|$ is most likely caused by small deviations in the provided physical parameters.

For the accurate simulation of jet pump flow fields, it is important that the boundary conditions do not introduce any steady flow component. By calculating the timeaveraged volume flow rate $U_{2}$ at each cross-section, it was found that for all cases simulated this value is less than $0.1 \%$ of the acoustic volume flow rate $U_{1}$. 

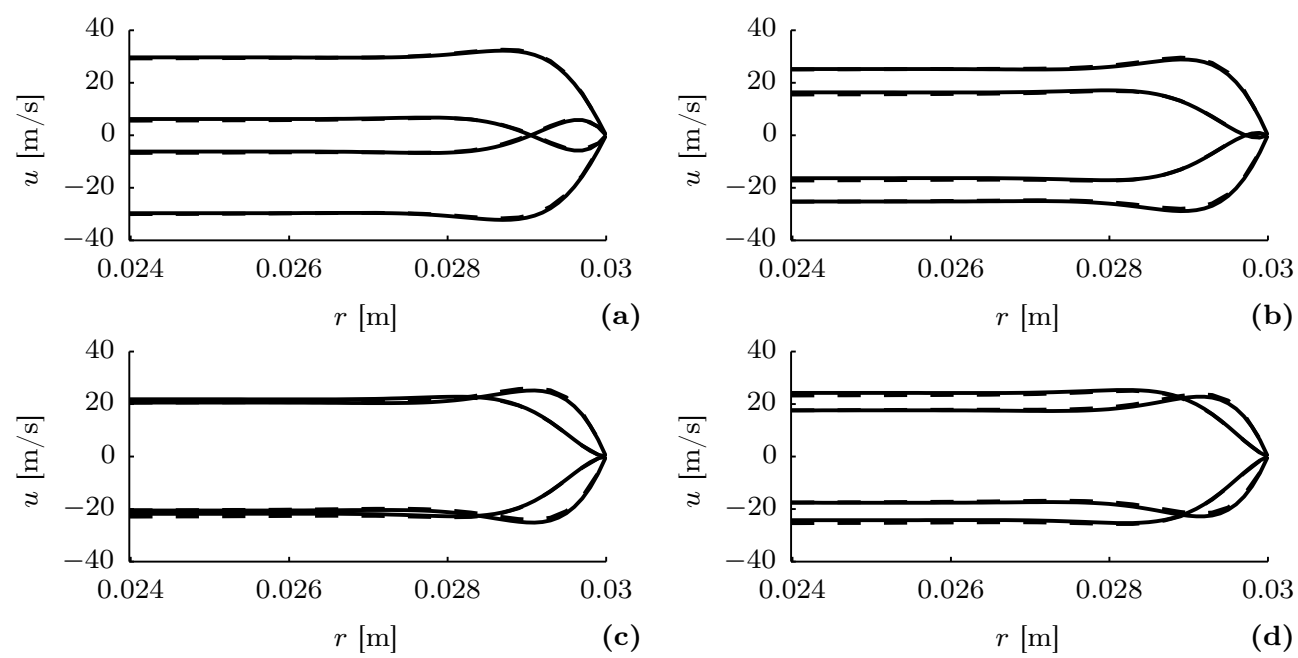

Figure A.2: Radial distribution of axial velocity component $u$ at four different axial locations: (a) $x=0.45 \mathrm{~m}$, (b) $x=1.025 \mathrm{~m}$, (c) $x=1.4 \mathrm{~m}$ and (d) $x=1.6 \mathrm{~m}$. Each graph contains four different time instances: $\varphi=0^{\circ}, 90^{\circ}, 180^{\circ}$ and $270^{\circ}$. Solid line indicates low-reduced frequency solution, dashed lines indicate simulation results. The graphs only show the region near the solid boundaries: $r=\left\langle R_{0}-10 \cdot \delta_{\nu}, R_{0}\right\rangle$. Specified pressure amplitude $\left|p_{1}\right|=5 \mathrm{kPa}$.
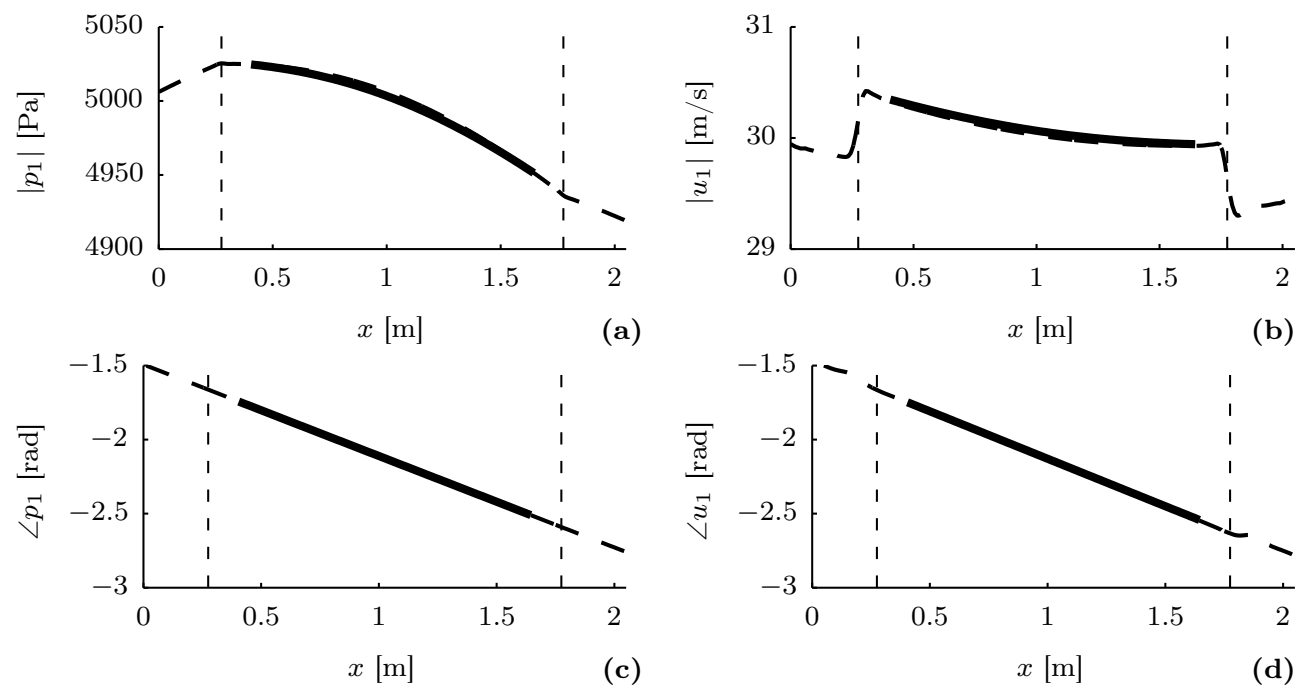

Figure A.3: (a) Pressure and (b) velocity amplitudes and (c- $\mathbf{d}$ ) phase angles against axial coordinate. Specified pressure amplitude $\left|p_{1}\right|=5 \mathrm{kPa}$. Solid line represents low-reduced frequency solution, dashed line indicates simulation results. The transition between the slip to the no-slip region is indicated with thin vertical lines. 


\section{Pressure measurement and calibration}

For the pressure measurements inside the experimental setup, piezoresistive differential pressure sensors (Honeywell 26PCAFA6D) are used. These sensors are mounted in the experimental setup using a custom 3D printed mount (see Figure B.1). This part is mounted flush with the inner wall of the setup and the curvature of the bottom surface matches the curvature of the tube to reduce the influence of these sensors on the flow field. The sensor membrane itself is situated approximately $17 \mathrm{~mm}$ inside the sensor housing and a $\varnothing 2.0 \mathrm{~mm}$ capillary is used to sample the pressure. The back-pressure port is exposed to the ambient pressure.

Smith and Swift investigated the effect of a capillary or pressure tap to sample the pressure. ${ }^{118}$ The authors observed streaming in the capillary and jetting at the capillary entrance which had a significant effect on their measurements of time-averaged pressure. To estimate this effect for the capillary inside the sensor housing of the Honeywell piezoresistive sensors, a two-dimensional CFD simulation has been carried out. The capillary is connected to a cavity which represents a part of the tube of the experimental setup. Oscillatory flow cases have been simulated at different pressure amplitudes up to $\left|p_{1}\right|=3 \mathrm{kPa}$. A very weak recirculation zone develops just inside the capillary and is observed for all different simulation cases. However, no jetting is observed as was reported by Smith and Swift at "Reynolds numbers much greater than unity", ${ }^{118}$ whereas the acoustic Reynolds numbers investigated range from 0.7

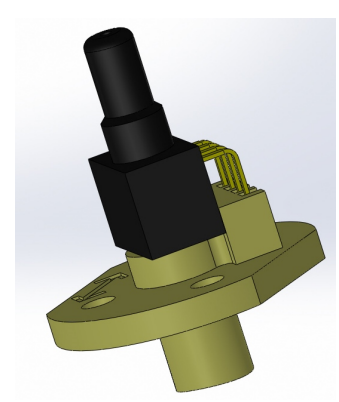

Figure B.1: Pressure sensor mount with sensor. 
to 22. By comparing the transient pressure signal from the CFD results in the center of the tube with that at the top of the capillary (where the sensor membrane is located), a negligible difference was found for the current pressure mount design. Hence, no measurement inaccuracies are expected to be introduced by the sensor mount design.

\section{B.1 Signal sampling}

The pressure sensor signal is amplified using a stabilized signal amplifier build around a TL052 op-amp. ${ }^{119}$ After setting the amplification factor and offset value to a fixed value (approximately $1 \mathrm{mV} / \mathrm{Pa}$ ), the sensors are calibrated using a dynamic pressure calibration setup (see Section B.2).

The pressure sensor signals are measured together with the loudspeaker voltage and current measurements using a National Instruments PCI-6250 data acquisition system. This device is capable to sample multi-channel signals up to $1.00 \mathrm{MS} / \mathrm{s}$ aggregate. Using four pressure sensors and two channels for voltage and current measurements, the maximum sample rate is $160 \mathrm{kS} / \mathrm{s}$. However, this maximum sample rate cannot be achieved due to the high source impedance of the loudspeaker voltage measurement $(>1 \mathrm{k} \Omega)$. Measuring this signal together with the (low impedance) pressure signals leads to ghosting: due to capacitive effects in the device multiplexer, signals on different channels are affected by each other when using a too high sample rate. Hence, the sample rate is reduced to $20 \mathrm{kS} / \mathrm{s}\left(f_{s}=20 \mathrm{kHz}\right)$ to avoid these effects and still obtain sufficient signal resolution.

As the data acquisition device uses one $\mathrm{AC} / \mathrm{DC}$ converter circuit for all channels, the signals are not sampled exactly simultaneous. There is a delay between the different channels, dependent on the sample rate. To compensate for this delay and synchronize the different signals in time, a LabVIEW procedure to align different waveforms is used. This ensures that the calculated phase difference between signals is exact and not dependent on the sampling rate.

After acquiring the pressure signals by the data acquisition system, the signals are further processed using a LabVIEW routine which is illustrated in Figure B.2. First of all, the signal is converted from a voltage to a pressure signal using the calibration values (see Section B.2) of the individual sensors. Then the acquired signal is Fourier transformed to obtain the complex amplitude at the specified driving frequency of the system and time-averaged to obtain the mean pressure. This procedure is repeated for every second of measurement data. For all results presented, 60 consecutive samples of $1 \mathrm{~s}$ are taken and subsequently averaged.

\section{B.2 Calibration}

The pressure sensors are calibrated against a reference sensor using a dynamic pressure calibration setup. This calibration setup consists of a loudspeaker (Monacor SP- 


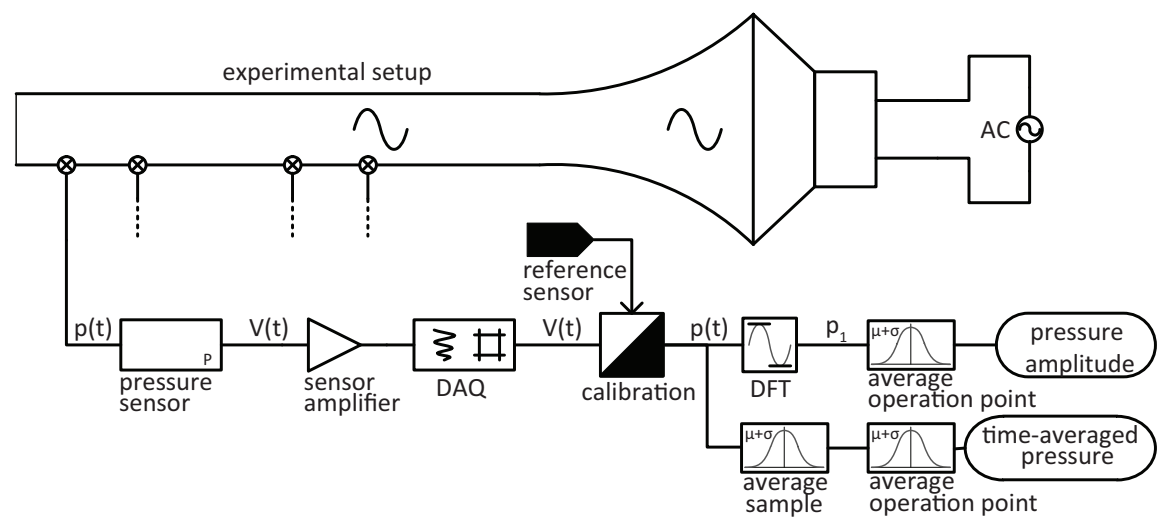

Figure B.2: Illustration of pressure measurement system. Consecutive blocks illustrate pressure sensor, sensor amplifier, data acquisition system and further data processing in LabVIEW routine.

$60 / 8$ ) that generates an acoustic wave inside a tube of $L=200 \mathrm{~mm}$. At the end of the tube three pressure sensors are mounted in plane: one reference sensor and two uncalibrated pressure sensors. The reference sensor is a calibrated Kulite XTE-190M pressure sensor. All sensors are calibrated using a sweep over frequency and pressure amplitude in a frequency range of $f=20 \mathrm{~Hz}$ to $150 \mathrm{~Hz}$ and a pressure amplitude range of $\left|p_{1}\right|=100 \mathrm{~Pa}$ to $2500 \mathrm{~Pa}$. For every setpoint (constant frequency and pressure amplitude), a set of 20 measurements is carried out. The data is acquired at a sample rate of $f_{s}=20 \mathrm{kHz}$ as described in Section B.1 and sampled for one second for every measurement.

From this calibration both the sensor sensitivity and the phase difference with the reference sensor are calculated. The sensor zero offset needs to be reset in situ before carrying out a measurement as it is observed that mounting and unmounting the sensors can lead to a slight change in zero offset caused by stressing the sensor membrane. Hence, it is not accurate to calibrate this zero offset in the calibration setup. The sensor sensitivity and phase correction are calculated in the following paragraphs. For both parameters outliers were observed when calibrating at a frequency of $f=50 \mathrm{~Hz}$ or $f=150 \mathrm{~Hz}$, probably due to interference with the electrical grid. Hence, the data sets at these frequencies are excluded from further processing.

\section{B.2.1 Sensor sensitivity}

By comparing the measured voltage amplitude from the uncalibrated sensor against the measured pressure amplitude from the reference sensor, the sensor sensitivity can be calculated,

$$
s=\frac{V_{\text {meas }}}{p_{1, \text { ref }}}[\mathrm{mV} / \mathrm{Pa}] .
$$

A linear least squares fit is applied to all sampled values to obtain the average sensor sensitivity. In Table B.1 the average sensor sensitivities for the four calibrated sensors 


\begin{tabular}{lllll}
\hline Sensor & \multicolumn{3}{l}{ Sensitivity $\bar{s} \pm \sigma[\mathrm{mV} / \mathrm{Pa}]$} & Time delay $\Delta t[\mathrm{~s}]$ \\
\hline P1 & 1.209 & \pm & $8.12 \cdot 10^{-4}$ & $1.69 \cdot 10^{-5} \mathrm{~s}$ \\
P2 & 0.907 & \pm & $7.64 \cdot 10^{-4}$ & $8.03 \cdot 10^{-6} \mathrm{~s}$ \\
P3 & 1.122 & \pm & $4.04 \cdot 10^{-4}$ & $1.39 \cdot 10^{-5} \mathrm{~s}$ \\
P4 & 1.091 & \pm & $5.32 \cdot 10^{-4}$ & $1.49 \cdot 10^{-5} \mathrm{~s}$ \\
\hline
\end{tabular}

Table B.1: Typical sensor sensitivity $s$ with standard deviation $\sigma$ and time delay $\Delta t$ for sensor P1-P4 (dated: 13-02-2015).

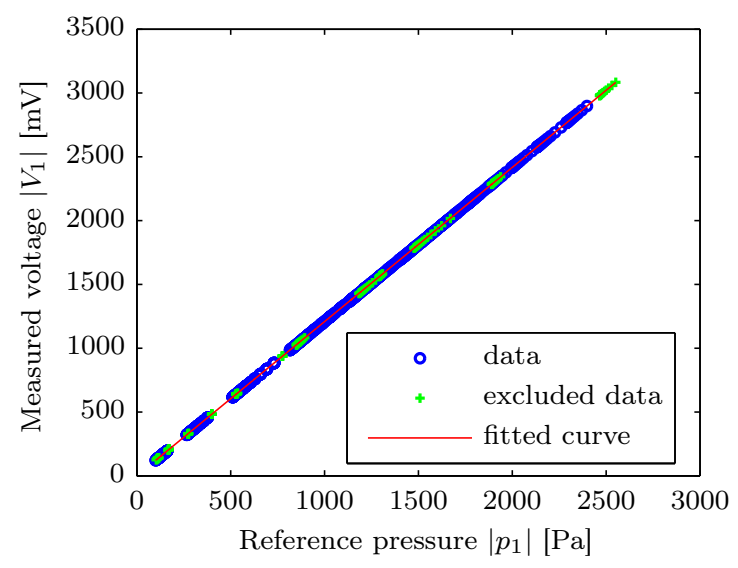

Figure B.3: Linear calibration curve for sensor P1 (dated: 13-02-2015).

are shown. A typical calibration curve is shown in Figure B.3. Note that due to the sensor stability these values differ from the exact values used in the results presented (see Sec. B.2.4).

\section{B.2.2 Phase correction}

As a second calibration parameter, the difference in phase between the uncalibrated sensor and reference sensor is calculated,

$$
\Delta \varphi=\varphi_{\text {ref }}-\varphi_{\text {meas }}[\mathrm{deg}] .
$$

For all sensors a clear linear relationship between phase difference and frequency is observed (see Figure B.4). This means there is a constant time delay present between the Kulite reference sensor and the Honeywell uncalibrated sensors,

$$
\Delta t=\frac{\Delta \varphi[\mathrm{rad}]}{\omega} .
$$

This time delay is estimated using a linear least squares fit and shown in Table B.1 for the four calibrated sensors. The largest difference in time delay is between sensors $\mathrm{P} 1$ and P2 and yields a phase error of $\Delta \varphi=0.32^{\circ}$ at $100 \mathrm{~Hz}$. Because the mutual 


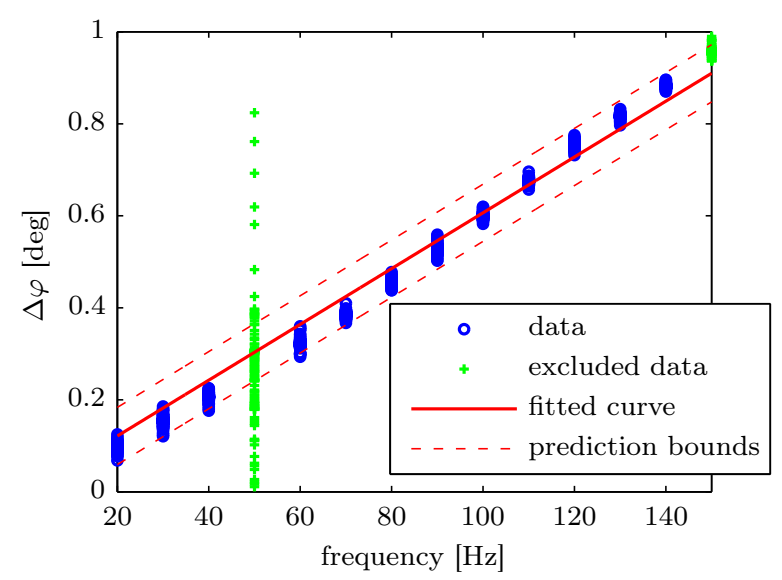

Figure B.4: Phase difference for sensor P1 with linear fit (dated: 13-02-2015).

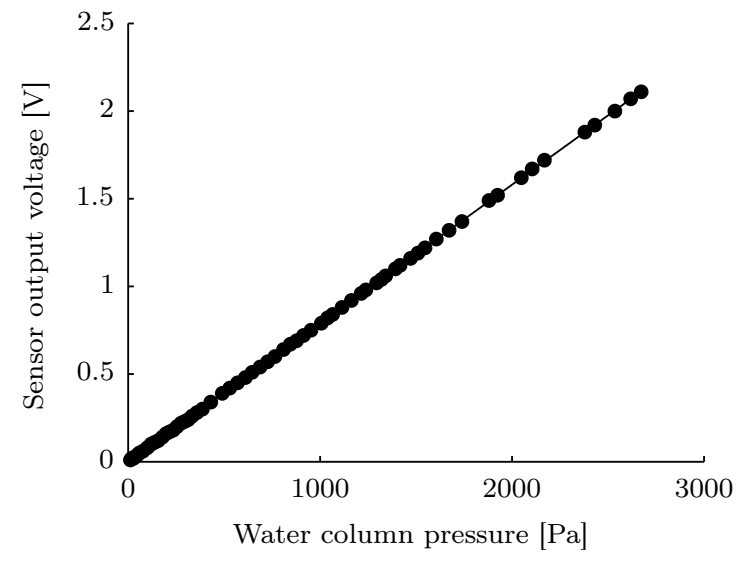

Figure B.5: Sensor linearity (sensor P1, dated: 02-12-2015). Line represents linear fit.

differences are so small, it is decided to not correct for this time delay in measurements and take it into account as a source of error.

\section{B.2.3 Linearity}

The linearity of the Honeywell piezoresistive sensors is investigated using a static calibration procedure. A pressure difference is generated using controlled flow through a hot-wire calibration nozzle. ${ }^{100}$ The pressure drop across the nozzle is measured simultaneously using a Honeywell pressure transducer and a water column with a resolution of $1 \mathrm{~Pa}$. The resulting relation between the measured water column pressure and the sensor output voltage is shown in Fig. B.5. The maximum difference with a linear fit is $\pm 0.1 \%$ of the full-scale range, which is considered acceptable and well within the sensor specification. 


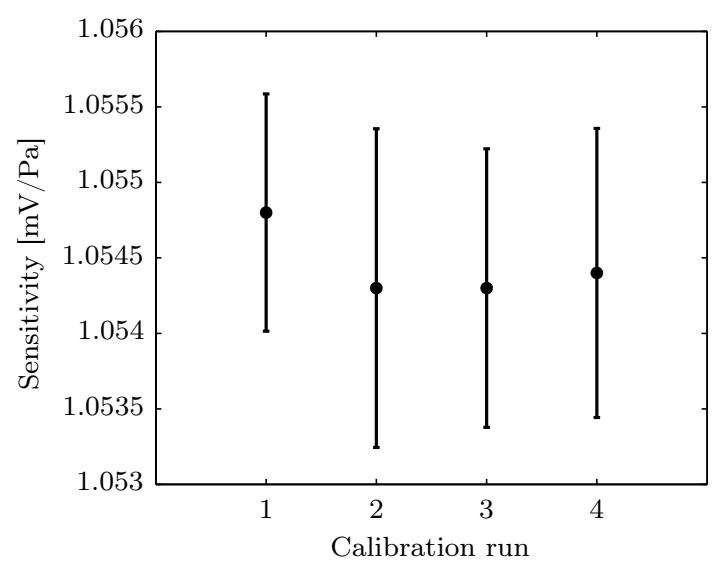

Figure B.6: Sensor sensitivity of four successive runs (sensor P1, dated: 10-01-2014). Error bars show $\pm \sigma$.

\section{B.2.4 Stability and repeatability}

To determine the stability of the Honeywell piezoresistive sensors, the dynamic calibration procedure has been repeated with an interval of approximately two months. A significant difference between the two measured sensor sensitivities is observed of $1.51 \%$. Hence, the pressure sensors are recalibrated on a regular basis before experiments are carried out.

The repeatability of the calibration procedure is quantified by performing multiple calibration runs after each other using the same set of sensors. In between each run, the sensors were removed from the calibration setup and repositioned and the measurement system was restarted. Figure B.6 shows the measured sensor sensitivities for the successive runs of one sensor. It is clear that all deviations in sensor sensitivity fall within one standard deviation distance from the measured values. This ensures the repeatability of the calibration procedure and sensors. 


\section{APPENDIX}

\section{Acoustic modeling of experimental setup}

\section{C.1 Introduction}

The velocity amplitude or acoustic displacement amplitude in the jet pump waist is one of the key parameters in determining the jet pump performance. However, in the thermoacoustic experimental setup no non-invasive velocity measurement can be performed at this location. In the following appendix, a linear acoustic model will be introduced that can be used to estimate the velocity amplitude in the jet pump waist based on the signal measured by the pressure sensors.

\section{C.2 Model}

To compute the relation between the velocity field inside the jet pump and the pressure at the sensor locations, a combination of acoustic models is used. The tube sections with constant cross-section left and right of the jet pump, are modeled using a one-dimensional low reduced frequency model. ${ }^{82,120}$ To calculate the acoustic field in the jet pump region, the thermoacoustic equations are solved in two dimensions using COMSOL Multiphysics v4.4 with the thermoacoustics or pressure acoustics module. ${ }^{121}$ In both models, air at $T_{0}=20^{\circ} \mathrm{C}$ and $p_{0}=1 \mathrm{~atm}$ is used as a fluid. Using this coupled approach, the computational load is significantly reduced compared to a COMSOL model of the full domain. This allows for the three-dimensional modeling of jet pump geometries with multiple holes, such as the ones discussed in Chapter 6.

Figure C.1 shows a schematic of the modeling approach. On the left boundary of the COMSOL model, an acoustic impedance is imposed from the low reduced frequency solution while on the right boundary of the COMSOL model a fixed pressure amplitude is specified. The pressure sensors are indicated with P1-P4. The horn 


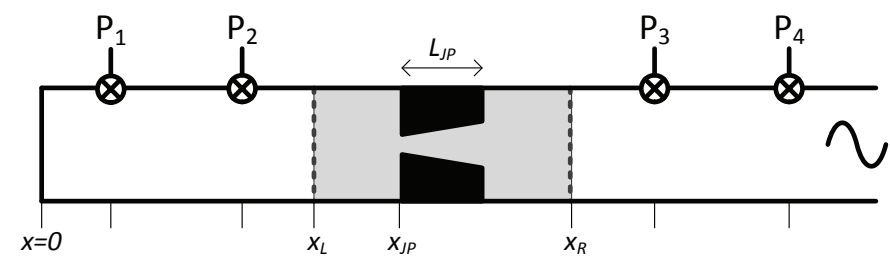

Figure C.1: Schematic of modeling the experimental setup in its standing wave configuration. The gray area indicates the computational domain bounded by the dashed lines at $x=x_{L}$ and $x=x_{R}$.

and loudspeaker section are located to the right and are not shown in the illustration.

\section{C.2.1 Low reduced frequency model}

The tube sections of the experimental setup are modeled using a one-dimensional low reduced frequency solution. The pressure and volume flow rate distribution in a tube are given by ${ }^{15}$

$$
\begin{aligned}
\frac{d p_{1}}{d x} & =-\frac{i \omega \rho_{0}}{\left(1-f_{\nu}\right) A} U_{1} \\
\frac{d U_{1}}{d x} & =-\frac{i A \omega}{\rho_{0} c_{0}^{2}}\left(1+(\gamma-1) f_{\kappa}\right) p_{1} .
\end{aligned}
$$

For a tube segment of length $\Delta x$, Eq. C.1-C.2 can be solved and are rewritten to a linear system of equations,

$$
\left(\begin{array}{c}
p_{1, \text { out }} \\
U_{1, \text { out }}
\end{array}\right)=[T]\left(\begin{array}{c}
p_{1, \text { in }} \\
U_{1, \text { in }}
\end{array}\right)
$$

where $U_{1}=u_{1} A$ is the volume flow rate and $[T]$ is a transfer matrix given by

$$
[T]=\left[\begin{array}{cc}
\cos (k \Delta x) & -\sin (k \Delta x) \frac{i \omega \rho_{0}}{\left(1-f_{\nu}\right) k A} \\
-\sin (k \Delta x) \frac{i\left(1-f_{\nu}\right) k A}{\omega \rho_{0}} & \cos (k \Delta x)
\end{array}\right] .
$$

In this transfer matrix, $f_{\nu}$ and $f_{\kappa}$ are the viscous and thermal thermoacoustic functions, respectively, and are dependent on the tube geometry. The complex wave number is defined as

$$
k=\frac{\omega}{c_{0}} \sqrt{\frac{1+(\gamma-1) f_{\kappa}}{1-f_{\nu}}} .
$$

Because the tube radius is large compared to the viscous and thermal penetration depths $(R / \delta>30)$, the boundary layer approximation is used to calculate the thermoacoustic functions. For a circular tube this yields

$$
\begin{aligned}
& f_{\nu}=(1-i) \delta_{\nu} / R \\
& f_{\kappa}=(1-i) \delta_{\kappa} / R
\end{aligned}
$$


In the following, the linear system in Eq. C.3 will be solved for the standing wave setup configuration with a closed end termination. The experimental setup with a closed end imposes a zero velocity boundary condition at the left side of the domain $(x=0$ in Figure C.1). Subsequently, the impedance at the left boundary of the COMSOL model $\left(x=x_{L}\right.$ in Figure C.1) can be specified. The system of equations (Eq. C.3) reduces to

$$
\left(\begin{array}{c}
p_{1, x=x_{L}} \\
U_{1, x=x_{L}}
\end{array}\right)=[T]_{L}\left(\begin{array}{c}
p_{1, x=0} \\
0
\end{array}\right) .
$$

where the locations $x=0$ and $x=x_{L}$ correspond to Figure C.1. Subsequently, the impedance at $x=x_{L}$ is given by

$$
Z_{L}=\frac{\left[T_{1,1}\right]_{L}}{\left[T_{1,2}\right]_{L}}=\frac{\cos (k \Delta x)}{-\sin (k \Delta x) \frac{i\left(1-f_{\nu}\right) k A}{\omega \rho_{0}}}=-\cot (k \Delta x) \frac{\omega \rho_{0}}{i\left(1-f_{\nu}\right) k A} .
$$

where $\Delta x=x_{L}$. The specific acoustic impedance at the boundary of the COMSOL model is defined as $z_{L}=-Z_{L} A$ to compensate for the boundary normal.

\section{C.2.2 Coupled COMSOL model}

The acoustic field in the jet pump region is computed using a two-dimensional axisymmetric acoustic simulation using COMSOL Multiphysics v4.4. ${ }^{121}$ Two different modeling approaches are investigated: either using a thermoacoustic model where the viscous boundary layer is fully resolved or applying a simplified pressure acoustics model where the viscous and thermal losses are estimated using the boundary layer approximation (Eq. C.6-C.7). The relation between the pressure amplitude at the sensor locations and the integrated velocity amplitude at the jet pump waist did not change significantly between the two models (see Fig. C.3 in Section C.3). As the computational load of the pressure acoustics model is substantially smaller than the thermoacoustics model, the former model is used for all further computations.

The computational domain consists of the jet pump and extends one jet pump length on either side of the jet pump as indicated in Figure C.1. On the outer wall of the computational domain, a sound hard wall boundary condition is imposed. The left boundary is defined by the specific impedance, $z_{L}=-Z_{L} A$, calculated in Section C.2.1. On the right boundary a fixed pressure amplitude is specified. As the acoustic model is linear, the actual amplitude does not matter and all other variables scale accordingly. A computational mesh with a maximum element size of $2 \mathrm{~mm}$ is used which is five times refined in the jet pump waist area. This resembles a total of 3600 elements for the jet pump sample with a $7^{\circ}$ taper angle.

\section{C.2.3 From measured pressure to velocity amplitude}

The described acoustic model can be used to estimate the velocity amplitude in the jet pump waist as a function of the pressure measured at the sensor locations. The linear 


\begin{tabular}{ll}
\hline Sensor & Position \\
\hline P1 & $50 \mathrm{~mm}$ \\
P2 & $350 \mathrm{~mm}$ \\
P3 & $860 \mathrm{~mm}$ \\
P4 & $1160 \mathrm{~mm}$ \\
\hline
\end{tabular}

Table C.1: Position of four pressure sensors with respect to the left side of the experimental setup $(x=0$ in Figure C.1).

conversion factor between the jet pump waist velocity amplitude and the pressure amplitude at any of the four sensors has the units of specific impedance,

$$
C_{i}=\frac{\left(p_{1}\right)_{P_{i}}}{u_{1, J P}},
$$

where $i$ denotes one of the four pressure sensors $\mathrm{P} 1-\mathrm{P} 4$. This conversion factor is a function of both frequency and jet pump position and is used in experiments to estimate the velocity amplitude in the jet pump waist while only the pressure amplitude at the sensor locations can be measured.

The velocity amplitude in the jet pump waist is directly derived from the solution of the COMSOL model by area-averaging the velocity amplitude over the waist radius. To calculate the pressure amplitudes at the specific sensor locations P1-P4, a similar approach as in Section C.2.1 is followed. The pressure amplitude at the sensor locations left of the jet pump can be calculated from the resulting pressure and velocity at the left boundary of the COMSOL domain, $p_{1, L}$ and $u_{1, L}$ by formulating a corresponding transfer matrix $[T]_{\left(P_{i} \rightarrow L\right)}$ where $\Delta x$ matches the distance from the sensor to the boundary of the COMSOL domain. Then the pressure follows from

$$
\left(p_{1}\right)_{P_{i}}=p_{1, L}\left[T_{2,2}\right]_{\left(P_{i} \rightarrow L\right)}-u_{1, L}\left[T_{1,2}\right]_{\left(P_{i} \rightarrow L\right)},
$$

where $i=1,2$ denotes the corresponding pressure sensor. For the pressure sensors on the right side of the jet pump, the pressure amplitude can be calculated from the pressure and velocity at the right boundary of the COMSOL domain, $p_{1, R}$ and $u_{1, R}$, with the corresponding transfer matrix $[T]_{\left(R \rightarrow P_{i}\right)}$. This yields

$$
\left(p_{1}\right)_{P_{i}}=p_{1, R}\left[T_{1,1}\right]_{\left(R \rightarrow P_{i}\right)}+u_{1, R}\left[T_{1,2}\right]_{\left(R \rightarrow P_{i}\right)},
$$

where $i=3,4$ denotes the corresponding pressure sensor. By substituting these calculated pressure amplitudes in Eq. C.10, the conversion factor based on either of the four pressure sensors is calculated.

\section{C.3 Results}

The COMSOL model coupled with the one-dimensional model is solved for a series of frequencies and jet pump locations. Furthermore, the influence of the jet pump 

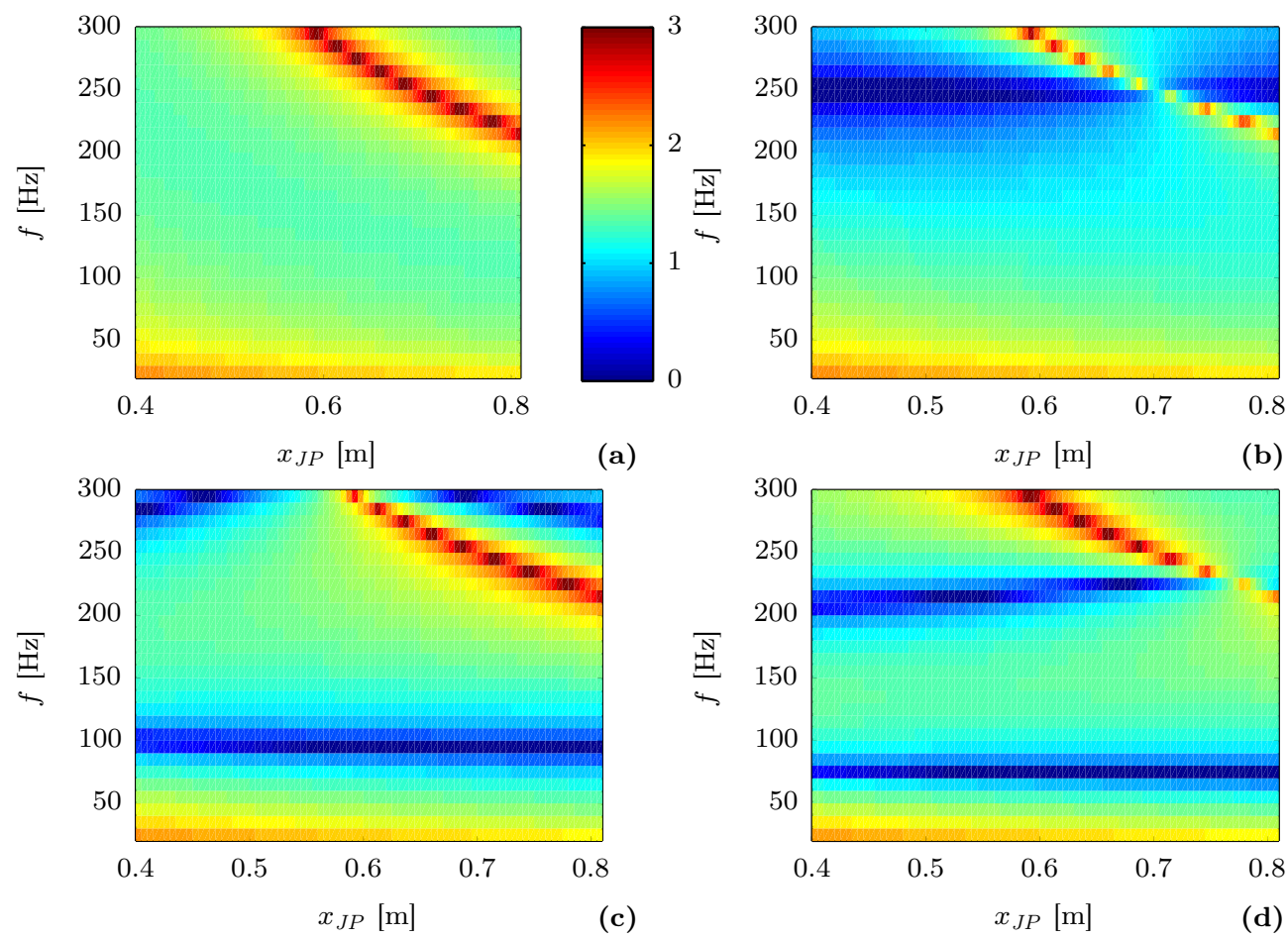

Figure C.2: $(\mathbf{a}-\mathbf{d})$ Conversion factor $C_{i}$ calculated based on the four different pressure sensors P1 - P4 as a function of the jet pump position $x_{J P}$ (horizontal axis) and frequency (vertical axis). Standing wave setup configuration and a jet pump sample with a single hole and $7^{\circ}$ taper angle used. Colors represent logarithm of conversion factor.

geometry on the conversion factor is investigated by changing the taper angle as well as the number of holes to cover all used jet pump samples. Results are presented for fixed sensor positions as indicated in Table C.1. With this sensor configuration, the jet pump can be moved along the length of its measurement section which has a length of $L=410 \mathrm{~mm}$ and is mounted at $x=400 \mathrm{~mm}$. As such, the jet pump location $x_{J P}$ is varied from $400 \mathrm{~mm}$ to $810 \mathrm{~mm}$. The driving frequency is varied from $20 \mathrm{~Hz}$ to $300 \mathrm{~Hz}$.

Figure C.2 shows the conversion factor calculated for the locations of the four different pressure sensors, the color indicates $\log _{10}\left(C_{i} \mid\right)$. The jet pump sample with a $7^{\circ}$ taper angle and a single hole is included. The horizontal axis shows the jet pump position and the vertical axis shows the driving frequency. From these figures it becomes clear for which configurations a high gradient in the conversion factor exists and consequently the calculation of the jet pump velocity amplitude $u_{1, J P}$ will be most likely prone to errors as small deviations in the frequency or the jet pump position will lead to large changes in the conversion factor. The red colored bands for example, indicate configurations where resonance occurs on either side of the jet pump which should be avoided to reduce the sensitivity of $u_{1, J P}$ to both frequency and jet pump 

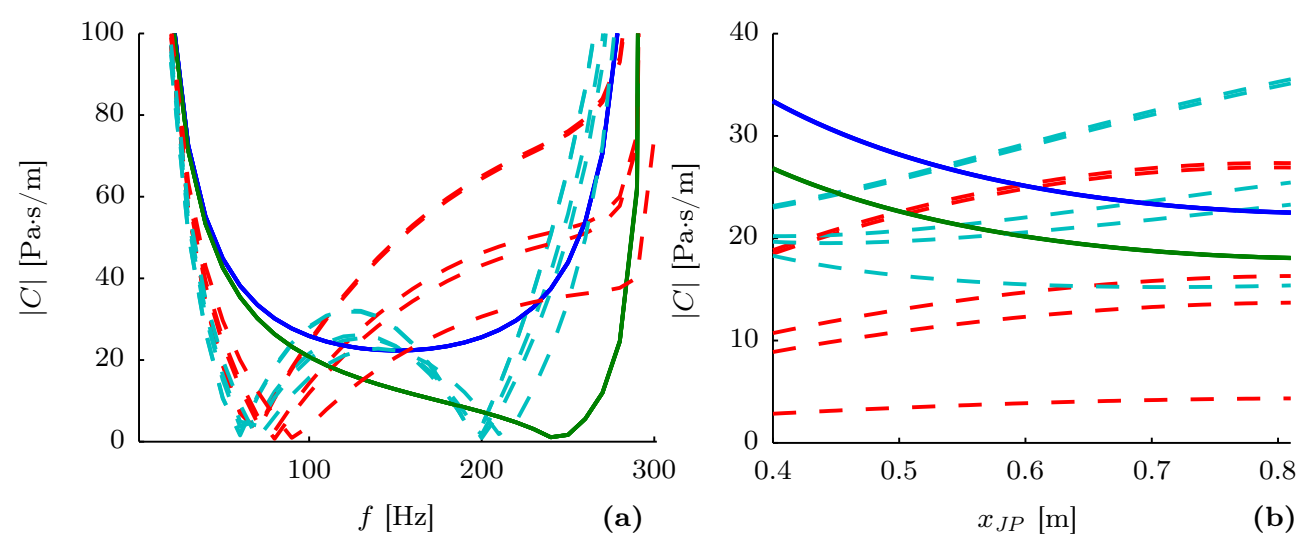

Figure C.3: Conversion factor based on the four different pressure sensor positions for four different jet pump samples ( $7^{\circ}$ samples with 1 and 4 holes and $15^{\circ}$ samples with 1 and 16 holes) as well as two different models (thermoacoustic model and pressure acoustics model) for the standing wave setup configuration. (a) Jet pump position fixed to $x_{J P}=572 \mathrm{~mm}$, variable frequency. (b) Frequency fixed to $f=100 \mathrm{~Hz}$, variable jet pump position. Blue and green solid lines show conversion factor based on P1 and P2, respectively. Red and cyan dashed lines show conversion factor based on P3 and P4 for the various samples and models.

location.

\section{C.3.1 Effect of jet pump geometry}

The influence of the jet pump geometry on the calculated conversion factor is investigated by both changing the taper angle as well as the number of holes. The taper angle is varied in the two-dimensional axisymmetric COMSOL model between $7^{\circ}$ and $15^{\circ}$. Varying the number of holes requires a fully three-dimensional COMSOL model which is implemented for the jet pump sample with four holes and a $7^{\circ}$ taper angle as well as for the jet pump sample with sixteen holes and a $15^{\circ}$ taper angle. A rotational symmetric slice of each geometry is simulated and symmetry boundary conditions are applied on the slice edges. A complete description of the geometry of the different jet pump samples can be found in Chapter 6 .

Figure C.3 shows the conversion factor as a function of frequency for all investigated geometries and the standing wave configuration with the jet pump position fixed to $572 \mathrm{~mm}$. Where a large spread in $\left|C_{3}\right|$ and $\left|C_{4}\right|$ is observed, the conversion factor based on P1 or P2 all overlay for the different geometries. The same is observed when studying the imaginary part of the conversion factor.

Both the taper angle as well as the number of holes do not influence the acoustic field on the small opening side of the jet pump (left side in Fig. C.1). Furthermore, as the total cross-sectional area of the jet pump waist is identical for all jet pump samples, the jet pump waist velocity is also identical among all samples. As a consequence, the conversion factor calculated from sensors $\mathrm{P} 1$ and $\mathrm{P} 2$ is not influenced by the jet pump sample while the conversion factor calculated from sensors P3 and P4 is sensitive to 

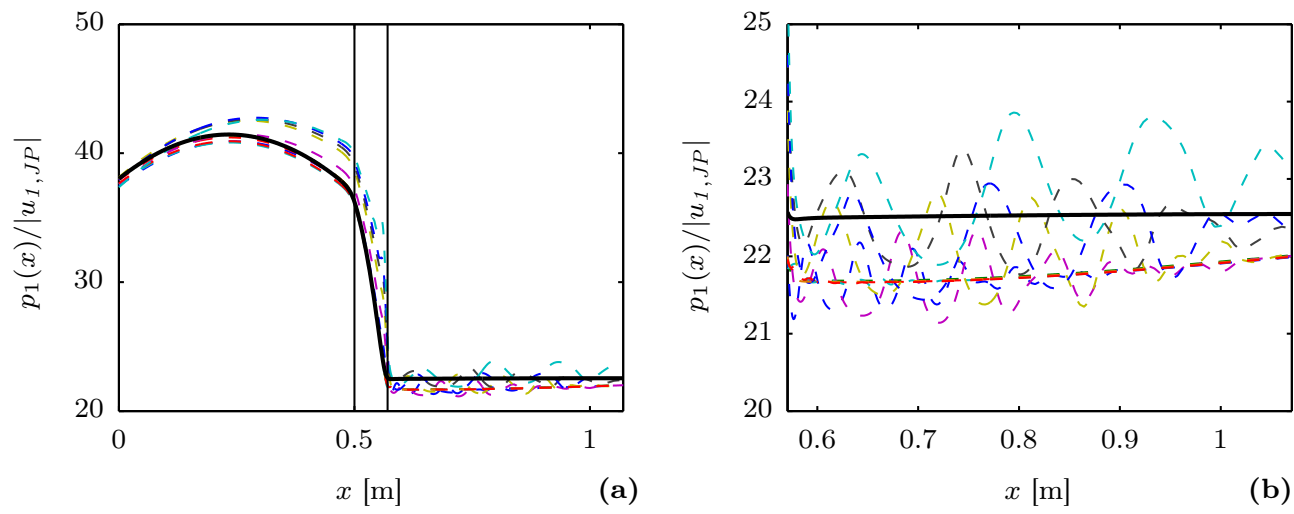

Figure C.4: Axial pressure amplitude $\left|p_{1}(x)\right|$ scaled with jet pump waist velocity $\left|u_{1, J P}\right|$ for the $7^{\circ}$ jet pump geometry and $f=100 \mathrm{~Hz}$. (a) Full computational domain and (b) close-up to the right side of the jet pump. Black line indicates acoustic model, colored lines represent results from various CFD simulations (see Chapter 2). Vertical thin lines indicate jet pump location.

a change in the jet pump geometry. Hence, in all experiments the jet pump waist velocity is based on either $C_{1}$ or $C_{2}$.

\section{C.4 Discussion}

One of the major assumptions in the acoustic model is its linearity. All higher order terms are neglected in the low reduced frequency approximation. Consequently, the time-averaged flow and pressure drop occurring due to the jet pump are not accounted for. In the following, an estimate of the accuracy of the acoustic model in predicting the jet pump waist velocity will be provided.

To investigate the influence of nonlinear effects on the calculated conversion factor $C_{i}$, the results from the acoustic modeling will be compared to (non-linear) CFD simulation results that were presented in Chapter 2-3. The acoustic model introduced in Section C.2 is adapted to match the setup of the CFD model. Instead of a coupled $1 \mathrm{D}$ and 2D model, now a fully two-dimensional model is employed and the boundary conditions are identical to what is specified in the CFD setup. On the left side of the domain ( $x=0$ in Fig. C.1) a velocity amplitude is specified and on the right side of the domain a pure traveling wave impedance is specified $\left(z_{R}=\rho_{0} c_{0}\right)$. The length of the tube sections on either side of the jet pump is set to $L_{i n}=500 \mathrm{~mm}$. Furthermore the jet pump orientation is reversed to comply with the CFD setup.

Figure C. 4 shows a comparison between the CFD results and results from the acoustic model. The pressure amplitude on the symmetry axis is scaled with the velocity amplitude in the jet pump waist representing the conversion factor for all $x$-locations, $|C(x)|$. Figure C.4a shows the full domain while in Fig. C.4b only the part to the right of the jet pump, upstream of the small jet pump opening, is shown. In Section C.3 it was explained that the conversion factor is most conveniently defined using the 
pressure amplitude from the domain after the small jet pump opening because of its independence on the jet pump geometry. When comparing the results from the acoustic model with the CFD results, it becomes clear that there is a maximum deviation of $3.8 \%$ in the region upstream of the small jet pump opening which is considered sufficiently accurate. The large spread in $|C(x)|$ for the various CFD cases, is due to strong vortex shedding. As there is only limited transient data available (five wave periods, $t=0.05 \mathrm{~s}$ ), the influence of the vortex shedding on the pressure field is not averaged out. In experiments the effect of vortex shedding on the pressure amplitude, and consequently on $C(x)$, is diminished due to a larger measurement time $(t=60 \mathrm{~s})$.

\section{C.5 Conclusion}

A combination of acoustic models is used to calculate a conversion factor from the pressure amplitude at the pressure sensor locations and the jet pump waist velocity. This conversion factor is shown to be independent of the jet pump geometry when based on a sensor that is located left to the jet pump $\left(x<x_{J P}\right)$. Lookup tables can be generated that provide the conversion factor as a function of driving frequency and jet pump position. Using this conversion factor, the jet pump waist velocity amplitude can be estimated based on the measured pressure amplitude. Operation points close to a resonance frequency of one of the tube sections should be avoided to reduce the sensitivity of the velocity amplitude estimation to small deviations in jet pump location or air conditions. 


\section{Effect of surface roughness on the jet pump performance}

\section{D.1 Introduction}

All experimental investigations in this research have been carried out using jet pump samples manufactured using a rapid prototyping laser sintering process and polished to a typical surface roughness ranging from $R_{A}=8.5 \mu \mathrm{m}$ to $12 \mu \mathrm{m}$. This is significantly higher than achieved with classical machining techniques where a surface roughness of $R_{A} \approx 1 \mu \mathrm{m}$ is common. Furthermore, in all CFD simulations performed in this work a perfect smooth surface is modeled. In order to estimate the effect the surface roughness can have on a jet pump's performance, experimental results obtained so far will be compared to results using a smooth metal jet pump sample.

An aluminum single hole jet pump sample with a $7^{\circ}$ taper angle is machined yielding a surface roughness of $R_{A}=1 \mu \mathrm{m}$. Measurements of time-averaged pressure drop and acoustic power dissipation at various frequencies and wave amplitudes have been carried out according to the methods described in Sec. 5.2. Figure D.1 shows a picture of both the jet pump samples investigated.

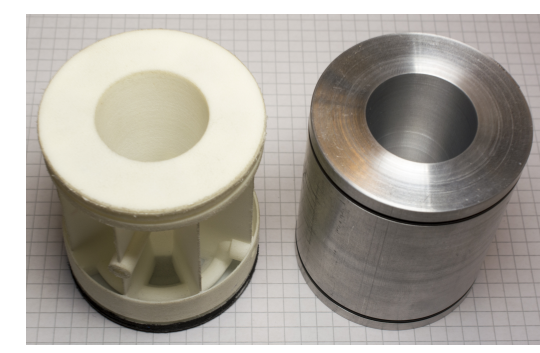

Figure D.1: Nylon (left) and aluminum (right) jet pump samples. 

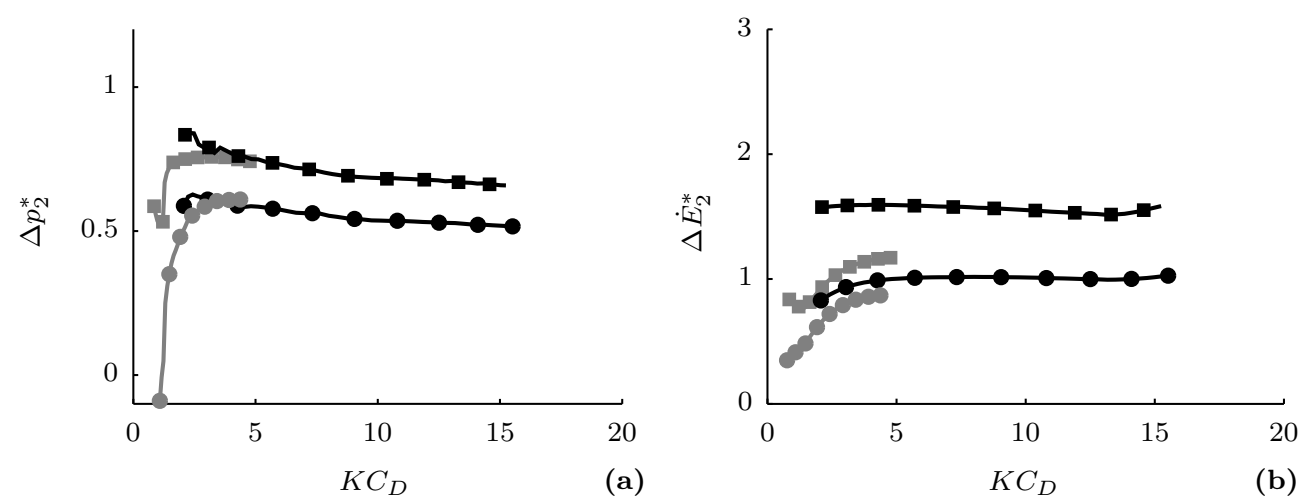

Figure D.2: (a) Dimensionless time-averaged pressure drop and (b) acoustic power dissipation measured using Nylon jet pump sample (ם) and aluminum jet pump sample (0) at $40 \mathrm{~Hz}$ (black) and $100 \mathrm{~Hz}$ (gray). Markers shown in one fifth of the measured points.

\section{D.2 Results}

Figure D.2 shows the dimensionless pressure drop and dimensionless acoustic power dissipation measured using two experimental samples: the original 3D printed Nylon sample (ם) and the aluminum sample $(\mathbf{\bullet})$. Both samples have an identical geometry, corresponding to the $7^{\circ}$ jet pump geometry introduced in Chapter 2. From Fig. D.2a it becomes clear that there is a constant difference in dimensionless pressure drop between the two different samples of $\Delta p_{2}^{*} \approx 0.15$. As the difference in pressure drop is already visible for low values of $K C_{D}$, it is not likely to be only caused by a difference in flow separation from the jet pump inner surface. Neither can the differences be fully attributed to a difference in the friction factor as for laminar flows the friction factor is not expected to be dependent on $R_{A} \cdot{ }^{15}$

Instead, the difference can possibly be associated with the minor losses generated by the expansion from the jet pump waist. It is conceivable that a rough surface results in more minor loss generation when the vortex rings roll up compared to a perfect smooth edge. ${ }^{122,123}$ The latter is backed up by observations from the dimensionless acoustic power dissipation (Fig. D.2b). Also for this performance indicator the smooth aluminum sample (0) shows a significant and consequent lower value than the 3D printed sample (ם). The difference is especially large at $40 \mathrm{~Hz}$ where the Reynolds number is lower for a given value of $K C_{D}$ compared to the $100 \mathrm{~Hz}$ case. The effect the Reynolds number has on the acoustic power dissipation is further discussed in Section 5.3. The acoustic power dissipation measured using the aluminum jet pump sample much better resembles the values obtained from CFD simulations, which can explain the differences experienced in the experimental validation in Section 4.4.3.

In the turbulent regime $\left(R e>R e_{c}\right)$, the values of both $\Delta p_{2}^{*}$ and $\Delta \dot{E}_{2}^{*}$ approach a constant value and can be averaged. From these averaged values, a total minor loss coefficient for both flow directions can be derived assuming the quasi-steady 


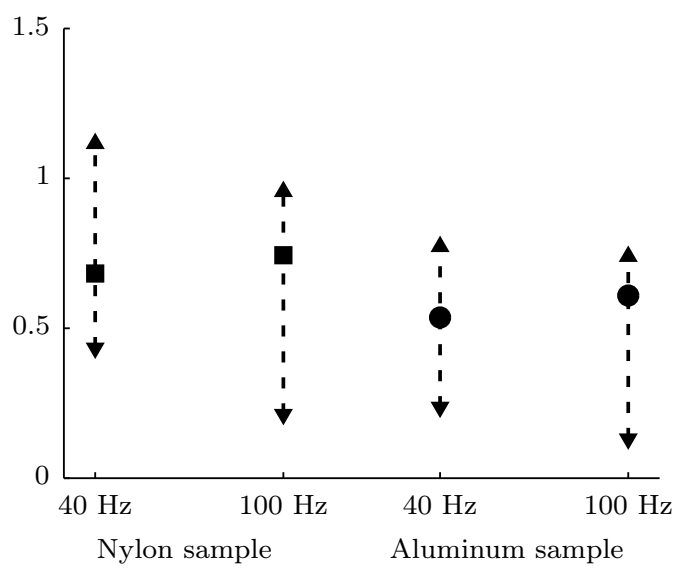

Figure D.3: Dimensionless pressure drop averaged for $R e>R e_{c}$ using Nylon jet pump sample $(\boldsymbol{\square})$ and aluminum jet pump sample ( ) . Values of average minor loss coefficients $K_{+}$and $K_{-}$ represented by $\boldsymbol{\Delta}$ and $\mathbf{\nabla}$, respectively.

approximation to be valid,

$$
\begin{aligned}
& K_{+}=1 / 2\left(\Delta \dot{E}_{2}^{*}+\Delta p_{2}^{*}\right) \\
& K_{-}=1 / 2\left(\Delta \dot{E}_{2}^{*}-\Delta p_{2}^{*}\right)
\end{aligned}
$$

with $K_{+}$representing the minor loss coefficient for flow from the big to the small jet pump opening (converging direction) and $K_{-}$representing the minor loss coefficient for flow from the small to the big opening (diverging direction). Figure D.3 shows these averaged minor loss coefficients together with the dimensionless pressure drop for the jet pump samples and frequencies investigated. This figure confirms that the differences between the two samples can be mainly attributed to a difference in $K_{+}$, i.e. to a difference in the minor losses generated at the expansion from the jet pump waist.

\section{D.3 Conclusion}

The presented results show that a reduction in surface roughness by a factor of ten can have a significant influence on a jet pump's performance. Although this investigation can explain certain differences between numerical and experimental results found so far, a more systematic study is required to determine the exact relation between surface roughness and jet pump performance. If jet pump flow phenomena such as flow separation and vortex shedding can indeed be influenced by a change in surface roughness, local roughness adaptations can become an additional degree of freedom in jet pump design optimization. 


\section{List of symbols}

\section{Abbreviations}

CFD Computational Fluid Dynamics

DNS Direct numerical simulation

HW Hot-wire anemometry probe

LES Large-eddy simulation

LODI Locally one-dimensional inviscid formulation (Appendix A)

LRM Linear relaxation method (Appendix A)

PSD Power spectral density

PWM Plane-wave masking (Appendix A)

TDIBC Time-domain impedance boundary condition (Appendix A)

URANS Unsteady Reynolds-averaged Navier-Stokes

\section{English letters}

A Cross-sectional area

A Boundary condition in low-reduced frequency solution (Eq. A.25)

$a_{i} \quad$ Filter coefficients in TDIBC (Eq. A.19)

$B \quad$ Boundary condition in low-reduced frequency solution (Eq. A.25)

$b_{j} \quad$ Filter coefficients in TDIBC (Eq. A.19)

C Fitting parameter or constant, see context

$C_{i} \quad$ Conversion from $p_{1}$ to $u_{1, J P}$ using sensor $P_{i} \quad$ (Eq. C.10)

$C_{p} \quad$ Specific heat at constant pressure

$c \quad$ Speed of sound

D Diameter

$d_{h} \quad$ Minimal center-to-center distance between adjacent jet pump holes

Dr Pressure drive ratio, $D r=p_{1} / p_{0}$

$\dot{E}_{2} \quad$ Acoustic power (Eq. 2.7)

$\Delta \dot{E}_{2} \quad$ Acoustic power dissipation across jet pump

$[\mathrm{Pa} \cdot \mathrm{s} / \mathrm{m}]$ $[\mathrm{J} / \mathrm{kg} \cdot \mathrm{K}]$ $[\mathrm{m} / \mathrm{s}]$

$\Delta \dot{E}_{2}^{*} \quad$ Dimensionless acoustic power dissipation (Eq. 2.11)

$f_{s} \quad$ Sample frequency of data acquisition system

$f_{\nu} \quad$ Viscous thermoacoustic function (Eq. C.6)

$f_{\kappa} \quad$ Thermal thermoacoustic function (Eq. C.7)

$f \quad$ Characteristic wave traveling towards TDIBC (Eq. A.16)

$g \quad$ Characteristic wave traveling from TDIBC

$[\mathrm{m} / \mathrm{s}]$

$g^{*} \quad$ External perturbation imposed by TDIBC (Eq. A.19) 
$i \quad$ Imaginary unit, $\sqrt{-1}$

$K \quad$ Minor loss coefficient

$K_{-} \quad$ Total minor loss coefficient in diverging direction

$K_{+} \quad$ Total minor loss coefficient in converging direction

$K \quad$ Relaxation factor in LRM (Eq. A.14)

$K C_{D} \quad$ Keulegan-Carpenter number based on jet pump diameter (Eq. 2.6)

$K C_{D, b} \quad K C_{D}$ at big jet pump opening (Eq. 3.3)

$K C_{L} \quad$ Keulegan-Carpenter number based on jet pump length (Eq. 2.5)

$K C_{\alpha} \quad K C_{D}$ corrected for the effect of the jet pump taper angle (Eq. 3.2)

$k \quad$ Complex wave number (Eq. C.5)

$L \quad$ Length

$L_{J P} \quad$ Total jet pump length (Eq. 2.1)

$\Delta L_{J P} \quad$ Size of jet pump transition length (Sec. 4.4.1)

$\mathcal{L}_{i} \quad$ Wave amplitude variation in LODI-formulation (Eq. A.6-A.10)

$\ell_{p} \quad$ Propagation distance

$\dot{m} \quad$ Mass flow rate

$n \quad$ Polytropic constant (Eq. A.27)

$N_{e l} \quad$ Number of elements in computational mesh

$N_{p} \quad$ Number of wave periods

$p \quad$ Pressure

$\Delta p_{2} \quad$ Time-averaged pressure drop across jet pump

$\Delta p_{2}^{*} \quad$ Dimensionless time-averaged pressure drop (Eq. 2.10)

$P_{i} \quad$ Reference to pressure sensor $i$ in experimental setup

$p_{\infty} \quad$ Far-field pressure outside computational domain

$p^{-} \quad$ Amplitude of backward traveling wave (Eq. A.23)

$p^{+} \quad$ Amplitude of forward traveling wave (Eq. A.23)

$\operatorname{Pr} \quad$ Prandtl number, $\operatorname{Pr}=\mu C_{p} / \kappa$

$\dot{Q} \quad$ Heat flux

R Reflection coefficient (Eq. A.21)

$r \quad$ Radial coordinate

$R_{0} \quad$ Outer tube radius (Fig. 1.5)

$R_{A} \quad$ Surface roughness average

$R_{b} \quad$ Radius of big jet pump opening (Fig. 1.5)

$R_{c} \quad$ Radius of curvature at small jet pump opening (Fig. 2.1)

$r_{h} \quad$ Hydraulic radius

$R_{s} \quad$ Effective radius of small jet pump opening (Fig. 2.1)

$R_{s}^{\prime} \quad$ Radius of small jet pump opening without curvature applied (Eq. 2.2)

$R_{\text {sep }} \quad$ Local jet pump radius where flow separation first occurs (Sec. 4.4.1)

$R_{t} \quad$ Radius of curvature of jet pump transition length (Sec. 4.4.1)

Re Acoustic Reynolds number (Eq. 2.8)

$R e_{c} \quad$ Critical Reynolds number for transition to turbulence (Eq. 2.9)

$R e_{D} \quad$ Acoustic Reynolds number based on diameter, $R e_{D}=\left|u_{1}\right| D \rho_{0} / \mu_{0}$

$S \quad$ Stokes number, $S=\sqrt{\omega D^{2} / \nu}$ 
$S_{u} \quad$ Area enclosed by $\left(\langle u\rangle,\left\langle u^{2}\right\rangle^{1 / 2}\right)$-loops in Fig. 5.9

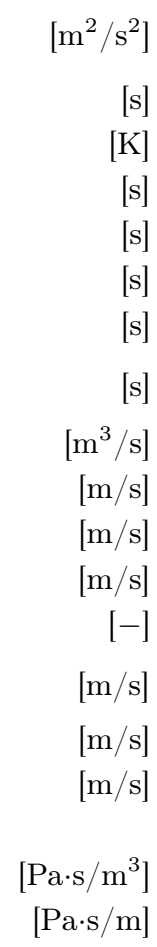

$\left[\mathrm{Pa} \cdot \mathrm{s} / \mathrm{m}^{3}\right.$ ]

$T \quad$ Period of acoustic wave, $T=1 / f$

$T$ Temperature

$T_{s} \quad$ Sampling time of data acquisition system

$t \quad$ Time

$t_{\max } \quad$ Total simulation time

$\Delta t \quad$ Time-step

$\Delta t_{p k} \quad$ Duration of velocity peak (time at which $\langle u\rangle>\overline{\langle u\rangle}+\left\langle u^{2}\right\rangle^{1 / 2}$ )

$U \quad$ Volume flow rate

$u \quad$ Axial velocity component

u Velocity field

$\mathbf{u}_{2} \quad$ Streaming velocity field (Eq. 2.3)

$\langle u\rangle \quad$ Phase-averaged velocity used in Chapter 5 (Eq. 5.6)

$\left\langle u^{2}\right\rangle^{1 / 2}$ Standard deviation of phase-averaged velocity (Eq. 5.10)

$v \quad$ Radial velocity component

$w \quad$ Azimuthal velocity component

$x \quad$ Axial coordinate

$Z \quad$ Acoustic impedance

$z \quad$ Specific acoustic impedance

\section{Greek letters}

$\alpha \quad$ Jet pump taper angle (half-angle, Fig. 2.1)

$\Gamma \quad$ Wave propagation constant (Eq. A.26)

$\gamma \quad$ Ratio of specific heats, $\gamma=C_{p} / C_{v}$

$\delta_{\nu} \quad$ Viscous penetration depth $\sqrt{2 \mu / \omega \rho}$

$\varepsilon \quad$ Relative error

$\eta \quad$ Jet pump effectiveness (Eq. 3.7)

$\eta \quad$ Axial coordinate in low-reduced frequency model, $\xi=\omega x / c_{0}$

$\lambda \quad$ Wavelength or eigenvalue

$\mu \quad$ Dynamic viscosity

$\xi_{1} \quad$ Acoustic displacement amplitude (Eq. 2.4)

$\xi \quad$ Radial coordinate in low-reduced frequency model, $\eta=r / R_{0}$

\section{Operators}

$|\ldots| \quad$ Absolute value 
$\langle\ldots\rangle \quad$ Time-average (Eq. 2.3), phase-average in Chapter 5 (Eq. 5.6)

$\nabla \times \mathbf{u}$ Curl operator, vorticity field

$\Delta \quad$ Difference

$\Im \quad$ Imaginary part of complex number

$J_{n} \quad$ Bessel function of the first kind with order $n$

.. $\quad$ Median in Chapter 5, area average in Appendix A

$\Re \quad$ Real part of complex number

$[T] \quad$ Transfer matrix (Eq. C.4)

$\widetilde{\text {.. Complex conjugate }}$

\section{Subscripts}

, Fluctuating part (Eq. 5.9)

* Dimensionless quantity

0 Reference value

1 Amplitude of first harmonic

2 Time-averaged value

$b \quad$ Big jet pump opening

$B L \quad$ Inside viscous boundary layer $\delta_{\nu}$

con Contraction

exp Expansion

FS Onset of flow separation

$i \quad$ Summation index

$J P \quad$ Jet pump

$L \quad$ Left boundary of computational domain

$p k \quad$ Peak value (Eq. 5.8)

$R \quad$ Right boundary of computational domain

reg Regenerator

$s \quad$ Small jet pump opening

spec Specified value

turb Onset of turbulence 


\section{Bibliography}

1. A. Glezer and M. Amitay. "Synthetic Jets." Annu. Rev. Fluid Mech., 34(1):503-529, 2002. doi:10.1146/annurev.fluid.34.090501.094913.

2. K. Marten, K. Shariff, S. Psarakos, and D. J. White. "Ring bubbles of dolphins." Sci. Am., 275(August):82-87, 1996. doi:10.1038/scientificamerican0896-82.

3. K. Shariff and A. Leonard. "Vortex Rings." Annu. Rev. Fluid Mech., 24:235-79, 1992. doi:10.1146/annurev.fl.24.010192.001315.

4. M. Gharib, E. Rambod, and K. Shariff. "A universal time scale for vortex ring formation." J. Fluid Mech., 360:121-140, 1998. doi:10.1017/S0022112097008410.

5. B. L. Smith and A. Glezer. "The formation and evolution of synthetic jets." Phys. Fluids, 10(9):2281, 1998. doi:10.1063/1.869828.

6. G. W. Swift, D. L. Gardner, and S. Backhaus. "Acoustic recovery of lost power in pulse tube refrigerators." J. Acoust. Soc. Am., 105(2):711-724, 1999. doi:10.1121/1.426262.

7. S. Backhaus and G. W. Swift. "A thermoacoustic-Stirling heat engine: detailed study." J. Acoust. Soc. Am., 107(6):3148-66, 2000. doi:10.1121/1.429343.

8. G. W. Swift and S. Backhaus. "A resonant, self-pumped, circulating thermoacoustic heat exchanger." J. Acoust. Soc. Am., 116(5):2923, 2004. doi:10.1121/1.1804634.

9. Lord Rayleigh. The theory of sound, Vol. 2. Dover, New York, 2nd edition, 1945. ISBN 9780486602936.

10. C. Sondhauss. "Über die Schallschwingungen der Luft in erhitzten Glasröhren und in gedeckten Pfeifen von ungleicher Weite." Ann. Phys., 155(1):1-34, 1850. doi:10.1002/andp.18501550102.

11. P. L. Rijke. "Notice of a new method of causing a vibration of the air contained in a tube open at both ends." Philos. Mag., 17(116):419-422, 1859.

12. A. Pożarlik. Vibro-acoustical instabilities induced by combustion dynamics In gas turbine combustors. Phd. thesis, University of Twente, 2010.

13. S. Backhaus, E. Tward, and M. Petach. "Traveling-wave thermoacoustic electric generator." Appl. Phys. Lett., 85(6):1085-1087, 2004. doi:10.1063/1.1781739.

14. C. M. de Blok, P. Owczarek, and M.-X. Francois. "Bi-directional turbines for converting acoustic wave power into electricity." In 9th PAMIR International Conference, pp. 433-438. CNRS - IPUL, Riga, Latvia, 2014.

15. G. W. Swift. Thermoacoustics: A Unifying Perspective for Some Engines and Refrigerators. ASA, Melville, NY, 2002. ISBN 0735400652. doi:10.1121/1.1561492.

16. N. Rott. "Damped and thermally driven acoustic oscillations in wide and narrow tubes." Z. Angew. Math. Me., 27(1):230-243, 1969. doi:10.1007/BF01595562.

17. N. Rott. "Thermoacoustics." Adv. Appl. Mech., 20:135-175, 1980. 
18. J. Wheatley, T. J. Hofler, G. Swift, and A. Migliori. "An intrinsically irreversible thermoacoustic heat engine." J. Acoust. Soc. Am., 74(1):153, 1983. doi:10.1121/1.389624.

19. S. Garrett and S. Backhaus. "The Power of Sound." Am. Sci., 88(6):516-525, 2000. doi:10.1511/2000.6.516.

20. P. H. Ceperley. "A pistonless Stirling engine - the traveling wave heat engine." $J$. Acoust. Soc. Am., 66(5):1508-1513, 1979. doi:10.1121/1.383505.

21. C. M. de Blok and N. A. H. J. van Rijt. "Thermo-acoustic system.", 2001.

22. S. Backhaus and G. W. Swift. "A thermoacoustic Stirling heat engine." Nature, 399:335-338, 1999. doi:10.1038/20624.

23. P. Owczarek and C. M. de Blok. "Details and experimental results of a stand-alone container cooled by a solar driven multi-stage traveling wave thermoacoustic system." In 3rd International Workshop on Thermoacoustics. University of Twente, Enschede, 2015. doi:10.3990/2.303.

24. J. J. Wollan, G. W. Swift, S. Backhaus, and D. L. Gardner. "Development of a Thermoacoustical Natural Gas Liquifier." In 2002 AIChE meeting, pp. 1-8. AIChE, New Orleans, LA, 2002.

25. C. M. de Blok. "Low operating temperature integral thermo acoustic devices for solar cooling and waste heat recovery." In Acoustics '08, pp. 3545-3550. ASA, Paris, France, 2008. doi:10.1121/1.2934526.

26. R.-1. Chen and S. L. Garrett. "A large solar/heat-driven thermoacoustic cooler." J. Acoust. Soc. Am., 108(5):2554, 2000. doi:10.1121/1.4743482.

27. A. Ruziewicz, C. M. de Blok, and P. Owczarek. "The analysis of hybrid solar powered cooling/heating system with the travelling-wave thermoacoustic refrigerator." In $3 r d$ International Workshop on Thermoacoustics. University of Twente, Enschede, 2015. doi: $10.3990 / 2.308$.

28. M. E. H. Tijani and S. Spoelstra. "A hot air driven thermoacoustic-Stirling engine." Appl. Therm. Eng., 61:866-870, 2013. doi:10.1016/j.applthermaleng.2013.04.052.

29. D. L. Gardner and C. Q. Howard. "Waste-Heat-Driven Thermoacoustic Engine and Refrigerator." In Acoustics 2009, pp. 23-26. AAS, Adelaide, Australia, 2009.

30. M.-X. Francois, C. M. de Blok, P. Bouakhao, M. Niphon, J. P. Thermeau, B. Guestin, L. Courtes, and D. Clodic. "The VALTA project: Full scale conversion of CHP engine flue gas heat into electricity." In 3rd International Workshop on Thermoacoustics. University of Twente, Faculty of Engineering Technology, Enschede, 2015. doi:10.3990/2.307.

31. M. E. H. Tijani and J. A. Lycklama à Nijeholt. "Bench scale electrically driven thermoacoustic heat pump." In 3rd International Workshop on Thermoacoustics. University of Twente, Enschede, 2015. doi:10.3990/2.310.

32. E. Tward, M. Petach, and S. Backhaus. "Thermoacoustic Space Power Converter." In AIP Conf. Proc., volume 654, pp. 656-661. AIP, Albuquerque, NM, 2003. doi:10.1063/1.1541352.

33. S. L. Garrett, J. A. Adeff, and T. J. Hofler. "Thermoacoustic refrigerator for space applications." J. Thermophys. Heat Tr., 7(4):595-599, 1993. doi:10.2514/3.466.

34. M. S. Haberbusch, C. T. Nguyen, J. C. Ickes, and G. W. Swift. "High-Power Thermoacoustic Stirling Heat Engine Results." In 9th Annual International Energy Conversion Engineering Conference, pp. 107-115. AIAA, San Diego, CA, 2011. 
35. A. Alemany, M.-X. Francois, C. M. de Blok, J. P. Roux, G. Poli, E. Zeminiani, E. Gaia, P. Jeantet, E. Roy, C. Chillet, J. Freiberg, R. Nikoluskins, G. Gerbeth, and S. Eckert. "Space thermoacoustic radio-isotopic power system: SpaceTRIPS." In 3rd International Workshop on Thermoacoustics. University of Twente, Enschede, 2015. doi: $10.3990 / 2.284$.

36. C. Lawn. "Development of a thermoacoustic travelling-wave engine." Proceedings of the Institution of Mechanical Engineers, Part A: Journal of Power and energy, 2013. doi:10.1177/0957650913498080.

37. Z. Yu, A. J. Jaworski, and S. N. Backhaus. "Design of a Low-Cost Thermoacoustic Electricity Generator and Its Experimental Verification." In 10th Biennial Conference on Engineering Systems Design and Analysis. ASME, Istanbul, Turkey, 2010. doi:10.1115/ESDA2010-24598.

38. S. L. Garrett and M. E. Poese. "There's (still) plenty of room at the bottom." Appl. Therm. Eng., 61(2):884-888, 2013. doi:10.1016/j.applthermaleng.2013.04.038.

39. C. M. de Blok. "On the design of near atmospheric air operated thermoacoustic engines." Technical report, Aster Thermoacoustics, 2013.

40. M. E. Poese, R. W. Smith, S. L. Garrett, R. van Gerwen, and P. Gosselin. "Thermoacoustic refrigeration for ice cream sales." In 6th Gustav Lorentzen Natural Working Fluids Conference, pp. 1-8. IIR, Glasgow, UK, 2004.

41. R. M. Keolian, M. E. Poese, R. W. M. Smith, E. C. Mitchell, C. M. Roberts, and S. L. Garrett. "Trillium: an inline thermoacoustic-stirling refrigerator." In 3rd International Workshop on Thermoacoustics. University of Twente, Enschede, 2015. doi: $10.3990 / 2.315$.

42. G. W. Swift. "Thermoacoustic natural gas liquefier." In US DOE Natural Gas Conference. DOE, Houston, Texas, 1997.

43. P. S. Spoor and G. W. Swift. "Thermoacoustic separation of a He-Ar mixture." Phys. Rev. Lett., 85(8):1646-9, 2000. doi:10.1103/PhysRevLett.85.1646.

44. W. V. Slaton and J. C. H. Zeegers. "An aeroacoustically driven thermoacoustic heat pump." J. Acoust. Soc. Am., 117(6):3628-3635, 2005. doi:10.1121/1.1904423.

45. D. McKelvey, S. Ballaster, and S. Garrett. "Shipboard electronics thermoacoustic cooler." In 130th Meeting of the Acoustical Society of America, p. 2961. ASA, St. Louis, Missouri, 1995. doi:10.1121/1.414007.

46. R. A. Ali, S. L. Garrett, J. A. Smith, and D. K. Kotter. "Thermoacoustic Thermometry for Nuclear Reactor Monitoring." IEEE Instrum. Meas. Mag., 16(3):18-25, 2013. doi:10.1109/MIM.2013.6521130.

47. S. L. Garrett. "Self-powered thermoacoustic sensors for nuclear reactors." In 3rd international workshop on thermoacoustics, University of Twente, 26-27 October 2015. University of Twente, Enschede, 2015. doi:10.3990/2.317.

48. G. W. Swift. "Thermoacoustic Engines and Refrigerators." Phys. Today, 48(7):22, 1995. doi:10.1063/1.881466.

49. S. L. Garrett. "Resource Letter: TA-1: Thermoacoustic engines and refrigerators." Am. J. Phys., 72(1):11, 2004. doi:10.1119/1.1621034.

50. T. Jin, J. Huang, Y. Feng, R. Yang, K. Tang, and R. Radebaugh. "Thermoacoustic prime movers and refrigerators: Thermally powered engines without moving components." Energy, 93:828-853, 2015. doi:10.1016/j.energy.2015.09.005. 
51. D. Gedeon. "DC Gas Flows in Stirling and Pulse Tube Cryocoolers." In R. G. Ross, editor, Cryocoolers 9, volume 9, chapter 7, pp. 385-392. Springer US, Boston, MA, 1997. doi:10.1007/978-1-4615-5869-9.

52. D. A. Wilcox. "personal communication.", 2015.

53. W. Kays and A. London. Compact heat exchangers. Krieger Publishing Company, Malabar, Florida, third edition, 1998. ISBN 9781575240602.

54. T. Biwa, Y. Tashiro, M. Ishigaki, Y. Ueda, and T. Yazaki. "Measurements of acoustic streaming in a looped-tube thermoacoustic engine with a jet pump." J. Appl. Phys., 101(6):064914, 2007. doi:10.1063/1.2713360.

55. M. S. Haberbusch, C. T. Nguyen, J. C. Ickes, and T. Y. Hui. " 4 kW Thermoacoustic Stirling Heat Engine Test Results." In 11th International Energy Conversion Engineering Conference, pp. 1-7. AIAA, San Jose, CA, 2013. doi:10.2514/6.2013-3809.

56. D. Wilcox and P. Spoor. "Gedeon streaming suppression in a small scale thermoacoustic-Stirling engine-generator." In 167th Meeting of the Acoustical Society of America, volume 135, pp. 2408-2408. ASA, Providence, RI, 2014. doi:10.1121/1.4877977.

57. M. Iguchi, M. Ohmi, and K. Meagawa. "Analysis of free oscillating flow in a U-shaped tube." B. JSME, 25(207):1398-1405, 1982. doi:10.1299/jsme1958.25.1398.

58. I. Idel'chik. "Resistance to flow through orifices with sudden change in velocity and flow area." In A. Ginevskiy and A. Kolesnikov, editors, Handbook of Hydraulic Resistance, chapter 4, pp. 223-275. Begell House, New York, 4th edition, 2007. ISBN 9781567002515 .

59. R. S. Wakeland and R. M. Keolian. "Influence of velocity profile nonuniformity on minor losses for flow exiting thermoacoustic heat exchangers (L)." J. Acoust. Soc. Am., 112(4):1249-1252, 2002. doi:10.1121/1.1502896.

60. A. Petculescu and L. A. Wilen. "Oscillatory flow in jet pumps: Nonlinear effects and minor losses." J. Acoust. Soc. Am., 113(3):1282-1292, 2003. doi:10.1121/1.1543932.

61. J. P. Oosterhuis, S. Bühler, D. Wilcox, and T. H. van der Meer. "Computational fluid dynamics analysis of the oscillatory flow in a jet pump: the influence of taper angle." In 9th PAMIR International Conference, pp. 391-395. CNRS - IPUL, Riga, Latvia, 2014. doi:10.13140/2.1.3222.8640.

62. C. V. King and B. L. Smith. "Oscillating flow in a 2-D diffuser." Exp. Fluids, 51(6):1577-1590, 2011. doi:10.1007/s00348-011-1170-7.

63. E. M. Sparrow, J. P. Abraham, and W. J. Minkowycz. "Flow separation in a diverging conical duct: Effect of Reynolds number and divergence angle." Int. J. Heat Mass Tran., 52(13-14):3079-3083, 2009. doi:10.1016/j.ijheatmasstransfer.2009.02.010.

64. B. L. Smith and G. W. Swift. "Power dissipation and time-averaged pressure in oscillating flow through a sudden area change." J. Acoust. Soc. Am., 113(5):24552463, 2003. doi:10.1121/1.1564022.

65. U. Ingård and S. Labate. "Acoustic circulation effects and the nonlinear impedance of orifices." J. Acoust. Soc. Am., 22(2):211-218, 1950. doi:10.1121/1.1906591.

66. B. L. Smith and G. W. Swift. "A comparison between synthetic jets and continuous jets." Exp. Fluids, 34(4):467-472, 2003. doi:10.1007/s00348-002-0577-6.

67. D. J. Nani and B. L. Smith. "Effect of orifice inner lip radius on synthetic jet efficiency." Phys. Fluids, 24(115110):1-16, 2012. doi:10.1063/1.4767725. 
68. R. Holman, Y. Utturkar, R. Mittal, B. L. Smith, and L. Cattafesta. "Formation Criterion for Synthetic Jets." AIAA J., 43(10):2110-2116, 2005. doi:10.2514/1.12033.

69. P. Morris, S. Boluriaan, and C. Shieh. "Computational thermoacoustic simulation of minor losses through a sudden contraction and expansion." In 7th AIAA/CEAS Aeroacoustics Conference, pp. 1-11. AIAA, Maastricht, The Netherlands, 2001. doi:10.2514/6.2001-2272.

70. P. Morris, S. Boluriaan, and C. Shieh. "Numerical simulation of minor losses due to a sudden contraction and expansion in high amplitude acoustic resonators." Acta Acust. united Ac., 90(3):393-409, 2004.

71. S. Boluriaan and P. J. Morris. "Suppression of traveling wave streaming using a jet pump." In 41st AIAA Aerospace Sciences Meeting $\&$ Exhibit, pp. 1-11. AIAA, Reno, NY, 2003. doi:10.2514/6.2003-367.

72. K. Tang, Y. Feng, S. Jin, T. Jin, and M. Li. "Performance comparison of jet pumps with rectangular and circular tapered channels for a loopstructured traveling-wave thermoacoustic engine." Appl. Engergy, 148:305-313, 2015. doi:10.1016/j.apenergy.2015.03.092.

73. J. P. Oosterhuis, S. Bühler, D. Wilcox, and T. H. van der Meer. "A numerical investigation on the vortex formation and flow separation of the oscillatory flow in jet pumps." J. Acoust. Soc. Am., 137(4):1722-1731, 2015. doi:10.1121/1.4916279.

74. ANSYS. "ANSYS CFX, Release 14.5.", 2011.

75. P. C. H. Aben. High-Amplitude Thermoacoustic Flow Interacting with Solid Boundaries. Phd thesis, Technische Universiteit Eindhoven, 2010.

76. J. A. Lycklama à Nijeholt, M. E. H. Tijani, and S. Spoelstra. "Simulation of a travelingwave thermoacoustic engine using computational fluid dynamics." J. Acoust. Soc. Am., 118(4):2265-2270, 2005. doi:10.1121/1.2035567.

77. I. Nowak, S. Rulik, W. Wróblewski, G. Nowak, and J. Szwedowicz. "Analytical and numerical approach in the simple modelling of thermoacoustic engines." Int. J. Heat Mass Tran., 77:369-376, 2014. doi:10.1016/j.ijheatmasstransfer.2014.05.018.

78. ANSYS. "Governing equations." In ANSYS CFX-Solver Theory Guide, pp. 18-26. ANSYS Inc., Canonsburg, PA, 2012.

79. B. van der Poel. Time-domain impedance boundary conditions in computational fluid dynamics for use in thermoacoustic modeling. Msc. thesis, University of Twente, 2013. URL http://purl.utwente.nl/essays/67782.

80. A. Huber, P. Romann, and W. Polifke. "Filter-based time-domain impedance boundary conditions for CFD applications." In ASME Turbo Expo 2008: Power for Land, Sea and Air, pp. 1-11. ASME, Berlin, Germany, 2008. doi:10.1115/GT2008-51195.

81. R. Kaess, A. Huber, and W. Polifke. "A Time-Domain Impedance Boundary Condition for Compressible Turbulent Flow." In 14th AIAA/CEAS Aeroacoustics Conference, pp. 1-15. AIAA, Vancouver, Canada, 2008. doi:10.2514/6.2008-2921.

82. H. Tijdeman. "On the propagation of sound waves in cylindrical tubes." J. Sound Vib., 39:1-33, 1975. doi:10.1016/S0022-460X(75)80206-9.

83. M. Ohmi and M. Iguchi. "Critical Reynolds number in an oscillating pipe flow." Bulletin of JSME, 25(200):365-371, 1982. doi:10.1299/jsme1958.25.165.

84. D. S. Antao and B. Farouk. "High amplitude nonlinear acoustic wave driven flow fields in cylindrical and conical resonators." J. Acoust. Soc. Am., 134(2):917-932, 2013. doi: $10.1121 / 1.4807635$. 
85. J. P. Oosterhuis, S. Bühler, D. Wilcox, and T. H. van der Meer. "Jet pumps for thermoacoustic applications: design guidelines based on a numerical parameter study." J. Acoust. Soc. Am., 138(4):1991-2002, 2015. doi:10.1121/1.4929937.

86. E. M. Cherry, C. J. Elkins, and J. K. Eaton. "Geometric sensitivity of threedimensional separated flows." Int. J. Heat Fluid Flow, 29(3):803-811, 2008. doi:10.1016/j.ijheatfluidflow.2008.01.018.

87. Y. Tsui and C. Wang. "Calculation of laminar separated flow in symmetric twodimensional diffusers." J. Fluids Eng., 117(4):612-616, 1995. doi:10.1115/1.2817311.

88. M. Nabavi. "Three-dimensional asymmetric flow through a planar diffuser: Effects of divergence angle, Reynolds number and aspect ratio." Int. Commun. Heat Mass, 37(1):17-20, 2010. doi:10.1016/j.icheatmasstransfer.2009.08.006.

89. S. R. Fugal, B. L. Smith, and R. E. Spall. "Displacement amplitude scaling of a twodimensional synthetic jet." Phys. Fluids, 17(4):045103, 2005. doi:10.1063/1.1872092.

90. V. Michelassi, W. Rodi, and J. Zhu. "Testing a low-Reynolds number k-epsilon turbulence model based on direct simulation data." AIAA J., 31(9):1720-1723, 1993. doi:10.2514/3.11835.

91. M. C. Vidya. Oscillatory flow in jet pumps: setup design and experiments. Msc. thesis, University of Twente, 2014. URL http://purl.utwente.nl/essays/66992.

92. H. Schlichting. Boundary-layer theory. McGraw-Hill, New York, 6th edition, 1968. ISBN 9780070553293.

93. B. L. Smith. "Pressure recovery in a radiused sudden expansion." Exp. Fluids, 36(6):901-907, 2004. doi:10.1007/s00348-003-0773-z.

94. M. A. G. Timmer, J. P. Oosterhuis, S. Bühler, D. Wilcox, and T. H. van der Meer. "Characterization and reduction of flow separation in jet pumps for laminar oscillatory flows." J. Acoust. Soc. Am., 139(1):193-203, 2016. doi:10.1121/1.4939490.

95. A. Petculescu. Viscous losses in porous media and jet-pumps under oscillatory flow conditions. Phd thesis, Ohio University, 2002.

96. R. A. Despard and J. A. Miller. "Separation in oscillating laminar boundary-layer flows." J. Fluid Mech., 47(1):21, 1971. doi:10.1017/S0022112071000909.

97. R. Simpson. "A review of some phenomena in turbulent flow separation." J. Fluids Eng., 103:520-533, 1981. doi:10.1115/1.3241761.

98. J. P. Oosterhuis, A. A. Verbeek, S. Bühler, D. Wilcox, and T. H. van der Meer. "Flow separation and turbulence in jet pumps for thermoacoustic applications." Flow Turbul. Combust., under review.

99. A. M. Fusco, W. C. Ward, and G. W. Swift. "Two-sensor power measurements in lossy ducts." J. Acoust. Soc. Am., 91(4):2229-2235, 1992. doi:10.1121/1.403656.

100. A. A. Verbeek, R. C. Pos, G. G. M. Stoffels, B. J. Geurts, and T. H. van der Meer. "A compact active grid for stirring pipe flow." Exp. Fluids, 54(10):1594, 2013. doi:10.1007/s00348-013-1594-3.

101. D. F. Elger and R. L. Adams. "Dynamic hot-wire anemometer calibration using an oscillating flow." J. Phys. E Sci. Instrum., 22:166-172, 1989. doi:10.1088/0022$3735 / 22 / 3 / 008$.

102. F. Jebali Jerbi, G. Huelsz, and S. Kouidri. "Acoustic velocity measurements in resonators of thermoacoustic systems using hot-wire anemometry." Flow Meas. Instrum., 32:41-50, 2013. doi:10.1016/j.flowmeasinst.2013.03.005. 
103. N. Didden. "On the formation of vortex rings: rolling-up and production of circulation." Z. Angew. Math. Phys., 30, 1979. doi:10.1007/BF01597484.

104. R. Akhavan. "An investigation of transition to turbulence in bounded oscillatory Stokes flows Part 1. Experiments." J. Fluid Mech, 225:395-422, 1991. doi:10.1017/S0022112091002100.

105. S. B. Pope. Turbulent Flows. Cambridge University Press, 2000. ISBN 0521598869. doi:10.1088/1468-5248/1/1/702.

106. M. Hino and M. Sawamoto. "Experiments on transition to turbulence in an oscillatory pipe flow." J. Fluid Mech., 75:193-207, 1976. doi:10.1115/1.3243663.

107. M. Iguchi and M. Ohmi. "Transition to Turbulence in a Pulsatile Pipe Flow: Part 2, Characteristics of Reversing Flow Accompanied by Relaminarization." B. JSME, 25(208), 1982. doi:10.1299/jsme1958.25.1529.

108. P. Merkli and H. Thomann. "Transition to turbulence in oscillating pipe flow." J. Fluid Mech., 68:567-575, 1975. doi:10.1017/S0022112075001826.

109. P. Welch. "The use of fast Fourier transform for the estimation of power spectra: A method based on time averaging over short, modified periodograms." IEEE Trans. Audio Elec., 15(2):70-73, 1967. doi:10.1109/TAU.1967.1161901.

110. J. P. Oosterhuis, M. A. G. Timmer, S. Bühler, D. Wilcox, and T. H. van der Meer. "On the performance and flow characteristics of jet pumps with multiple orifices." $J$. Acoust. Soc. Am., under review.

111. M. Watson, A. J. Jaworski, and N. J. Wood. "A study of synthetic jets from rectangular and dual-circular orifices." Aeronaut. J., 107(1073):427-434, 2003.

112. B. L. Smith and A. Glezer. "Vectoring of Adjacent Synthetic Jets." AIAA J., 43(10):2117-2124, 2005. doi:10.2514/1.12910.

113. NIST/SEMATECH. e-Handbook of Statistical Methods. NIST/SEMATECH, 2013. URL http://www.itl.nist.gov/div898/handbook.

114. P. Zwart and J. Abshoff. "Non-reflective Boundary Conditions in CFX-5." Technical report, ANSYS, 2005.

115. T. Poinsot and S. Lele. "Boundary conditions for direct simulations of compressible viscous flows." J. Comput. Physics, 101:104-129, 1992. doi:10.1016/0021-9991(92)90227$\mathrm{P}$.

116. L. Selle, F. Nicoud, and T. Poinsot. "The actual impedance of non-reflecting boundary conditions: implications for the computation of resonators." AIAA J., 42(5):1-21, 2004. doi:10.2514/1.1883.

117. W. Polifke, C. Wall, and P. Moin. "Partially reflecting and non-reflecting boundary conditions for simulation of compressible viscous flow." J. Comput. Physics, 213(1):437-449, 2006. doi:10.1016/j.jcp.2005.08.016.

118. B. L. Smith and G. W. Swift. "Measuring second-order time-average pressure." J. Acoust. Soc. Am., 110(2):717, 2001. doi:10.1121/1.1382615.

119. C. M. de Blok. "Personal communication.", 2013.

120. F. J. M. van der Eerden. Noise reduction with coupled prismatic tubes. Phd thesis, University of Twente, 2000.

121. COMSOL. COMSOL Multiphysics v4.4 Reference Guide. COMSOL AB, Stockholm, Sweden, 2013. 
122. M. Dziedzic and H. Leutheusser. "An experimental study of viscous vortex rings." Exp. Fluids, 21(5), 1996. doi:10.1007/BF00189051.

123. P. W. Bearman and J. M. R. Graham. "Vortex shedding from bluff bodies in oscillatory flow: A report on Euromech 119." J. Fluid Mech., 99(2):225-245, 1980. doi:10.1017/S0022112080000596. 


\section{Curriculum vitae}

Joris Oosterhuis, born on November 15, 1987 in Breda, The Netherlands.

2016 - present Technologist Thermal in the Mechatronic Equipment department at Philips Innovation Services, Eindhoven.

$2012-2016 \quad$ PhD student in the department of Thermal Engineering at the Faculty of Engineering Technology, University of Twente. Thesis title: "Oscillatory flows in jet pumps: towards design guidelines for thermoacoustic applications".

$2009-2011 \quad$ MSc Mechanical Engineering at the University of Twente. Thesis title: "Recuperation in a $\mu$ CHP appliance". Graduated cum laude.

$2006-2010 \quad$ BSc Mechanical Engineering at the University of Twente.

$2000-2006$ Secondary school (VWO+) at the Zernike College in Haren. Graduated cum laude.

\section{Journal publications}

J. P. Oosterhuis, M. A. G. Timmer, S. Bühler, D. Wilcox, and T. H. van der Meer. "On the performance and flow characteristics of jet pumps with multiple orifices." Journal of the Acoustical Society of America, under review.

J. P. Oosterhuis, A. A. Verbeek, S. Bühler, D. Wilcox, and T. H. van der Meer. "Flow separation and turbulence in jet pumps for thermoacoustic applications." Flow, Turbulence and Combustion, under review.

M. A. G. Timmer, J. P. Oosterhuis, S. Bühler, D. Wilcox, and T. H. van der Meer. "Characterization and reduction of flow separation in jet pumps for laminar oscillatory flows." Journal of the Acoustical Society of America, 139(1):193-203, 2016. doi:10.1121/1.4939490.

J. P. Oosterhuis, S. Bühler, D. Wilcox, and T. H. van der Meer. "Jet pumps for thermoacoustic applications: design guidelines based on a numerical parameter study." Journal of the Acoustical Society of America, 138(4):1991-2002, 2015. doi:10.1121/1.4929937.

J. P. Oosterhuis, S. Bühler, D. Wilcox, and T. H. van der Meer. "A numerical investigation on the vortex formation and flow separation of the oscillatory flow in jet 
pumps." Journal of the Acoustical Society of America, 137(4):1722-1731, 2015. doi:10.1121/1.4916279.

S. Bühler, D. Wilcox, J. P. Oosterhuis, and T. H. van der Meer. "Mean temperature profile at the entrance of a thermoacoustic stacked screen heat exchanger." Magnetohydrodynamics, 51(3):609-616, 2015.

J. P. Oosterhuis, S. Bühler, D. Wilcox, and T. H. van der Meer. "Design of a concentric heat exchanger using computational fluid dynamics as a design tool." Computational Thermal Sciences, 5(2):165-176, 2013. doi:10.1615/ComputThermalScien.2013006317.

\section{Conference publications}

J. P. Oosterhuis, S. Bühler, D. A. Wilcox, and T. H. van der Meer. "Performance measurements of jet pumps with multiple holes." In 3rd International Workshop on Thermoacoustics. University of Twente, Enschede, The Netherlands, 2015. doi:10.3990/2.294.

M. A. G. Timmer, J. P. Oosterhuis, S. Bühler, D. A. Wilcox, and T. H. van der Meer. "Reducing flow separation in jet pumps." In 3rd International Workshop on Thermoacoustics. University of Twente, Enschede, The Netherlands, 2015. doi:10.3990/2.296.

M. C. Vidya, J. P. Oosterhuis, and T. H. van der Meer. "A traveling wave termination for a thermoacoustic setup." In 3rd International Workshop on Thermoacoustics. University of Twente, Enschede, The Netherlands, 2015. doi:10.3990/2.285.

J. P. Oosterhuis, S. Bühler, D. Wilcox, and T. H. van der Meer. "Computational fluid dynamics analysis of the oscillatory flow in a jet pump: the influence of taper angle." In 9th PAMIR International Conference, pp. 391-395. CNRS IPUL, Riga, Latvia, 2014. doi:10.13140/2.1.3222.8640.

S. Bühler, D. Wilcox, J. P. Oosterhuis, and T. H. van der Meer. "Mean temperature profile at the entrance of a thermoacoustic stacked screen heat exchanger." In 9th PAMIR International Conference, pp. 407-411. CNRS - IPUL, Riga, Latvia, 2014.

S. Bühler, D. Wilcox, J. P. Oosterhuis, and T. H. van der Meer. "Thermal entrance effects in a thermoacoustic stacked screen regenerator." In 15th International Heat Transfer Conference, pp. 1-9. 2014.

J. P. Oosterhuis, S. Bühler, D. Wilcox, and T. H. van der Meer. "Computational fluid dynamics simulation of Rayleigh streaming in a vibrating resonator." In Proceedings of Meetings on Acoustics, volume 19, pp. 030008-030008. ASA, Montréal, 2013. doi:10.1121/1.4799051.

S. Bühler, D. Wilcox, J. P. Oosterhuis, and T. H. van der Meer. "Calculation of thermoacoustic functions with computational fluid dynamics." In Proceedings of Meet- 
ings on Acoustics, volume 19, pp. 030003-030003. ASA, Montréal, Canada, 2013. doi:10.1121/1.4799088.

J. P. Oosterhuis, S. Bühler, D. Wilcox, and T. H. van der Meer. "CFD as a Design Tool for a Concentric Heat Exchanger." In 9th International Conference on Heat Transfer, Fluid Mechanics and Thermodynamics, pp. 110-115. Malta, 2012. doi:10.13140/2.1.3026.2563.

\section{Other publications}

T. H. van der Meer, P. Blanc-Benon, C. M. de Blok, and J. P. Oosterhuis, editors. Proceedings of the 3rd International workshop on thermoacoustics. University of Twente, Enschede, The Netherlands, 2015. ISBN 9789036539746. doi:10.3990/1.9789036539746. 


\section{Dankwoord}

De afgelopen vier jaar heb ik met veel enthousiaste, behulpzame, slimme en vriendelijke mensen mogen samenwerken. Ik kijk met veel plezier terug op mijn tijd als promovendus en ik ben iedereen erg dankbaar die op wat voor manier dan ook een bijdrage heeft geleverd aan de totstandkoming van dit boekje. Een aantal mensen wil ik in het bijzonder benoemen.

Allereerst wil ik graag mijn begeleider en promotor Theo van der Meer bedanken. Bedankt voor al het vertrouwen dat je in me hebt getoond en voor de feitelijke carte blanche voor het uitvoeren van mijn promotieonderzoek. Ook wanneer ik even niet zo goed wist welke richting het onderzoek uit zou gaan, bleef je geduldig en vol vertrouwen. Het hoogtepunt was misschien wel de Third International Workshop on Thermoacoustics die we spontaan bedachten om te gaan organiseren. Ik ben erg blij dat we op een succesvolle workshop kunnen terugkijken.

Although formally not my co-supervisor, I do not know of anyone who served this role better than Doug Wilcox. Your fascination for thermoacoustics has inspired me ever since we met during my internship at Bosch. Thanks a lot for making my PhD research possible and for the endless Skype conversations we had in the past years. You are an excellent motivator and your enthusiasm has kept the project going. Despite your busy agenda you were always willing to share your thoughts and give support. Thank you for all your efforts in making our publications better readable. And last but not least, thank you for all the fun we had together when we met "in real life". I wish you all the best and good luck with finalizing your own $\mathrm{PhD}$ research.

Dit onderzoek was niet mogelijk geweest zonder de financiële steun van Bosch Thermotechnology en Rijksdienst voor Ondernemend Nederland in het kader van het project "Sound Solution for Small Scale Residential Energy Conversion". Sjoerd Reijke, Anne de Jong, Ysbrand Wijnant en Kees de Blok, bedankt voor jullie input en de interesse in dit onderzoek. Daarnaast ben ik Jos Zeegers van de TU Eindhoven zeer erkentelijk voor het ter beschikking stellen van de experimentele opstelling. Dankzij deze opstelling zijn we in staat geweest de experimenten uit te voeren die in dit werk beschreven zijn. Uiteraard ook bedankt voor alle ondersteuning bij het opbouwen van de opstelling.

Een bijzonder woord van dank voor jou Simon. Ik heb veel respect voor jouw doorzettingsvermogen, waardoor ik dit nu ook in het Nederlands aan je kan schrijven. Als ik ergens niet uit kwam, had je altijd een goed idee paraat om net op een andere manier naar resultaten te kijken of een probleem op te lossen. Aan veel van het in dit proefschrift beschreven werk ligt een goed idee van jou ten grondslag, waarvoor 
ik je zeer dankbaar ben. Het was erg fijn om met je samen te werken. Minstens net zo dankbaar ben ik je voor alle gezelligheid op kantoor, tijdens onze reizen en ook daarbuiten. Joost en Anton, jullie wil ik ook bedanken voor al het sparren, onze "briljante" plannen en natuurlijk de gezelligheid tijdens en ook na kantooruren.

Een groep studenten heeft een belangrijke bijdrage geleverd aan dit onderzoek en ik heb de samenwerking erg motiverend gevonden. Daan, Bart, Citra, Rully, Sam, Frederieke, Koen and Adam: thank you for your contributions and the great cooperation. Michael, jou wil ik niet alleen heel erg bedanken voor je werk als afstudeerder maar uiteraard ook voor je hulp en inbreng als collega. Ik ben blij dat je het thermoakoestisch onderzoek voortzet en ik wens je veel succes.

De vakgroep zou heel wat minder soepel lopen zonder Sally en Henk-Jan. Sally, bedankt voor alle organisatorische en administratieve ondersteuning. Je staat altijd voor iedereen klaar en je hulp bij de organisatie van de Third International Workshop on Thermoacoustics was onmisbaar. Henk-Jan, jou wil ik graag bedanken voor de ondersteuning in het lab, de hulp bij het opbouwen van de experimentele opstelling en het mij bijbrengen van de nodige technische vaardigheden. Als we weer eens een wild idee hadden, dacht je mee of keek je op het goede moment de andere kant op.

Ook buiten de vakgroep zijn er velen bij wie ik altijd terecht kon voor advies, een luisterend oor of gewoon wat afleiding. Iedereen bedankt daarvoor! Robert-Jan, het zou je tekort doen om je alleen te bedanken voor alle koffie. Onze afspraken in de vroege ochtend waren altijd een goed begin van de dag en je adviezen over statistiek en het wederzijds klagen over reviews hebben me zeker geholpen. Fokke en Robert-Jan, bedankt voor alle gezelligheid. Het is gauw tijd voor een vervolg op ons uitstapje naar Legoland.

Ondanks dat dit haast als vanzelfsprekend voelt, wil ik ook graag mijn familie bedanken. Pappa, mamma en Suzanne, bedankt voor jullie betrokkenheid. Het feit dat ik dat altijd als "gewoon" heb kunnen ervaren, is best bijzonder. Oma, bedankt voor je interesse en de vastberadenheid om te willen begrijpen waar ik me mee bezig houd. Janita, heel erg bedankt dat je er voor me bent, zodanig dat ik me niet meer kan voorstellen hoe het zonder jou is.

Finally, I would like to thank you as a reader for reading this thesis and the interest in my work! 
Stellingen behorende bij het proefschrift

\section{OSCILLATORY FLOWS IN JET PUMPS}

Towards design guidelines for thermoacoustic applications

1. Stromingsloslating is een belangrijke oorzaak van prestatieverliezen in jet pompen en dient derhalve voorkomen te worden. - Hoofdstuk 3

2. Het oplossen van een numeriek model voor laminaire oscillerende stroming is op dit moment de meest effectieve methode om het globale gedrag van een turbulente oscillerende stroming te kunnen voorspellen.

- Hoofdstuk 3 en 5

3. De omzetting van akoestische energie naar elektrische energie is de grootste uitdaging voor de commerciële ontwikkeling van thermoakoestische motoren.

4. Studie versnellende maatregelen leiden tot risicomijdend gedrag bij studenten hetgeen ten koste gaat van hun nieuwsgierigheid en creativiteit.

5. Er is een discrepantie tussen de hoge prioriteit voor wetenschappelijke publicaties en de lage prioriteit voor het reviewen van deze publicaties. Deze discrepantie leidt tot onnodige vertraging van wetenschappelijk onderzoek.

6. De anonimiteit van reviews van wetenschappelijke publicaties komt de wetenschappelijke dialoog niet ten goede en leidt mede tot datgene gesteld in Stelling 5.

7. Het gebruik van de woorden "kwalitatief" en "kwantitatief" om een onderzoeksmethode aan te duiden is verwarrend zolang aan kwantitatief onderzoek een hogere kwaliteit wordt toegeschreven dan aan kwalitatief onderzoek.

8. Zodra van een fysisch principe de werking is aangetoond, kan een verbetering enkel verkregen worden door eerst de werking te begrijpen.

- Naar: "Fokke \&s Sukke weten waar het in de wetenschap om draait".

door Joris Oosterhuis, april 2016. 
Propositions accompanying the dissertation

OSCILLATORY FLOWS IN JET PUMPS

Towards design guidelines for thermoacoustic applications

1. Flow separation is a major cause of performance losses in jet pumps and should therefore be avoided.

- Chapter 3

2. Solving a numerical model for laminar oscillatory flows is currently the most effective method to predict the global behavior of a turbulent oscillatory flow.

- Chapter 3 and 5

3. The conversion of acoustic energy to electric energy is the largest remaining challenge for the commercial development of thermoacoustic engines.

4. Policies implemented to accelerate a student's program completion are causing risk aversion among students, which is at the expense of their curiosity and creativity.

5. A discrepancy exists between the high priority placed on scientific publications and the low priority to the reviewing of these publications. This discrepancy leads to an unnecessary delay in scientific research.

6. The anonymity of the reviewing process of scientific publications does not benefit the scientific dialogue and partially causes what is stated in Proposition 5.

7. The use of the words "qualitative" and "quantitative" to indicate a research method is confusing because often quantitative research is considered to be of higher quality than qualitative research.

8. Once a physical principle has been proven, further improvement can only be achieved after understanding its fundamentals.

- After: "Fokke $\&$ Sukke weten waar het in de wetenschap om draait".

by Joris Oosterhuis, April 2016. 